Supporting Information

\title{
A Boron Activating Effect Enables Cobalt-Catalyzed Asymmetric Hydrogenation of Sterically Hindered Alkenes
}

\author{
Peter Viereck, Simon Krautwald, Tyler P. Pabst, and Paul J. Chirik* \\ Department of Chemistry, Frick Laboratory \\ Princeton University, Princeton, NJ 08544, USA \\ pviereck@princeton.edu,pchirik@princeton.edu
}

\section{Table of Contents}

$\begin{array}{ll}\text { I. General Considerations } & \text { S2 }\end{array}$

II. Preparation of Boron Reagents and 1,1-Diboryl Alkenes S4

III. General Hydrogenation Procedure and 1,1-Diboryl Alkanes S22

IV. Functionalization of 1,1-Diboryl Alkane $\quad$ S37

V. Hydrogenation of Alkenyl Boronates $\quad$ S41

$\begin{array}{lr}\text { VI. Deuterium Labeling Studies } & \text { S58 }\end{array}$

$\begin{array}{lr}\text { References } & \text { S80 }\end{array}$

NMR Spectra and SFC Chromatograms of Isolated Compounds S81 


\section{General Considerations}

All air- and moisture-sensitive manipulations were carried out using vacuum line, Schlenk and cannula techniques or in an MBraun inert atmosphere (nitrogen) dry box unless otherwise noted. All glassware was stored in a pre-heated oven prior to use. The thick-walled glass vessel ("bomb") used in hydrogenation experiments was dried in a $200^{\circ} \mathrm{C}$ oven overnight and then connected to a high vacuum line ( $\mathrm{H}$ grease) and left to cool to $23^{\circ} \mathrm{C}$. The bomb was then heated with a heat gun for 4-5 minutes, and again left to cool to $23^{\circ} \mathrm{C}$. This was repeated twice, and then the bomb was sealed under vacuum with a Kontes valve and brought into the glovebox. ( $\left.{ }^{\mathrm{C} y} \mathrm{APDI}\right) \mathrm{CoMe}^{7}$, ${ }^{\mathrm{Cy}} \mathrm{CoMe}^{1},{ }^{\mathrm{tBu}} \mathrm{CoCM}^{2}$, and $\mathbf{P d}-\mathrm{G}^{3}{ }^{3}$ were prepared using literature procedures. The solvents used for air- and moisture-sensitive manipulations were dried and deoxygenated using literature procedures. $^{4}$

${ }^{1} \mathrm{H}$ NMR spectra were recorded on either Bruker ADVANCE 300 or 500 spectrophotometers operating at $300.13 \mathrm{MHz}$, and $500.46 \mathrm{MHz}$, respectively. ${ }^{13} \mathrm{C}$ NMR spectra were recorded on either Bruker ADVANCE 300 or 500 spectrometer operating at $75.48 \mathrm{MHz}$ and $125.85 \mathrm{MHz}$, respectively. ${ }^{11} \mathrm{~B}$ NMR spectra were recorded on either Bruker ADVANCE 300 operating at 96 $\mathrm{MHz}$. All ${ }^{1} \mathrm{H}$ and ${ }^{13} \mathrm{C}$ NMR chemical shifts are reported in ppm relative to $\mathrm{SiMe}_{4}$ using the ${ }^{1} \mathrm{H}$ (chloroform- $d: 7.26$ ppm; benzene- $d_{6}: 7.16 \mathrm{ppm}$ ) and ${ }^{13} \mathrm{C}$ (chloroform-d: $77.16 \mathrm{ppm}$; benzene- $d_{6}$ : $128.06 \mathrm{ppm}$ ) chemical shifts of the solvent as a standard. ${ }^{13} \mathrm{C}$ NMR signals are not observed for carbons attached to boron. ${ }^{1} \mathrm{H}$ NMR data for diamagnetic compounds are reported as follows: chemical shift, multiplicity $(\mathrm{s}=$ singlet, $\mathrm{d}=$ doublet, $\mathrm{t}=$ triplet, $\mathrm{q}=$ quartet, $\mathrm{p}=$ pentet, $\mathrm{br}=$ broad, $m=$ multiplet, $a p p=$ apparent, obsc $=$ obscured $)$, coupling constants $(\mathrm{Hz})$, integration, assignment . ${ }^{1} \mathrm{H}$ NMR data for paramagnetic compounds are reported as follows: chemical shift, integration, peak width at half height $(\mathrm{Hz}) .{ }^{13} \mathrm{C}$ NMR data for diamagnetic compounds are reported as follows: chemical shift, number of protons attached to carbon (e.g. $\left.\mathrm{CH}_{2}\right)$, assignment.

High-resolution mass spectra were obtained at Princeton University mass spectrometry facilities using an Agilent 6210 TOF LC/MS. 
Single crystals suitable for X-ray diffraction were coated with polyisobutylene oil, transferred to a nylon loop and then quickly transferred to the goniometer head of a Bruker SMART APEX DUO diffractometer equipped with a molybdenum X-ray tube $(\lambda=0.71073 \AA)$ and a Cu X-ray tube $(\lambda=1.54178 \AA)$. Preliminary data revealed the crystal system. The data collection strategy was optimized for completeness and redundancy using the Bruker COSMO software suite. The space group was identified, and the data were processed using the Bruker SAINT+ program and corrected for absorption using SADABS. The structures were solved using direct methods (SHELXS) completed by subsequent Fourier synthesis and refined by full-matrix least-squares procedures. 


\section{Preparation of Starting Materials and Reagents}

Preparation of BPin-BDan: Prepared using a modified procedure by Suginome et al. ${ }^{5}$ To a dry solution of 1,8-diaminonapthalene (3.96 g, $25 \mathrm{mmol})$ and pinacol $(2.95 \mathrm{~g}, 25 \mathrm{mmol})$ in DCM (ca. $50 \mathrm{~mL}$ ) was added tetrakis(dimethylamino)diboron $(4.95 \mathrm{~g}, 25 \mathrm{mmol})$. The dark red solution was cooled to $0{ }^{\circ} \mathrm{C}$, and $2 \mathrm{M} \mathrm{HCl}(0.25 \mathrm{mmol}, 0.125 \mathrm{~mL})$ was added dropwise to the stirring solution. The reaction mixture was allowed to warm to $\mathrm{rt}$, and stirred under $\mathrm{N}_{2}$ for $48 \mathrm{~h}$. The mixture was filtered through a pad of silica, eluting with DCM. The solvent was removed in vacuo. The dark purple solid was recrystallized in Ethyl Acetate/Hexanes, affording $2.45 \mathrm{~g}, 25 \%$ yield of BPinBDan as off white crystals (the slight purple color can be completely removed from further recrystallizations under inert atmosphere, however this was not necessary for the chemistry reported - and no major impurities were observed by NMR analysis). Spectroscopic data match that obtained in the literature.

Preparation of Ethynyl-BDan: (2-ethynyl-2,3-dihydro-1H-naphtho[1,8-de][1,3,2]diazaborinine)

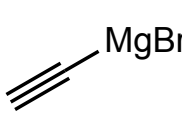

$$
\begin{aligned}
& \text { 1) } \mathrm{B}(\mathrm{OMe})_{3}, \mathrm{THF},-78^{\circ} \mathrm{C}-\mathrm{rt}, 1 \mathrm{~h} \\
& \hline \text { 2) } \mathrm{HCl},-78{ }^{\circ} \mathrm{C}-\mathrm{rt}, 1 \mathrm{~h} \\
& \text { 3) Diaminonapthalene, rt, overnight }
\end{aligned}
$$

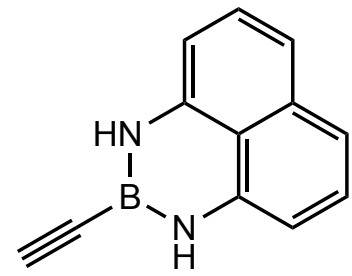

To a septum capped round bottom flask was added a solution of ethynylmagnesium bromide (20 $\mathrm{mL}, 0.5 \mathrm{M}, 1$ equiv.) and dry THF (c.a. $20 \mathrm{~mL})$, and cooled to $-78^{\circ} \mathrm{C}$. Trimethyl borate $(2.04 \mathrm{~mL}$, $10 \mathrm{mmol}, 1$ equiv.) was added dropwise, and the solution was allowed to warm to room temperature. During this time, a white precipitate forms formed, and solution was rapidly stirred for 1 hour. The solution was again cooled to $-78^{\circ} \mathrm{C}$, a solution of $\mathrm{HCl} / \mathrm{Ether}(5 \mathrm{~mL}, 2 \mathrm{M}, 1$ equiv.) was added dropwise, and the solution warmed to room temperature, resulting in a clear solution. The solution was stirred for $1 \mathrm{~h}$, and solid 1,8-diaminonapthalene was added, and stirred at room temperature for 6 hours. The mixture was filtered through a plug of silica gel, and eluted with DCM. The solvent was removed in vacuo, affording spectroscopically pure Ethynyl-BDan (2- 
ethynyl-2,3-dihydro-1H-naphtho[1,8-de][1,3,2]diazaborinine) as an off-white solid $93 \%, 1.76 \mathrm{~g}$. The spectra recorded match that obtained in the literature. ${ }^{6}$

General Procedure A - for preparation of 1,1-diboryl alkenes:

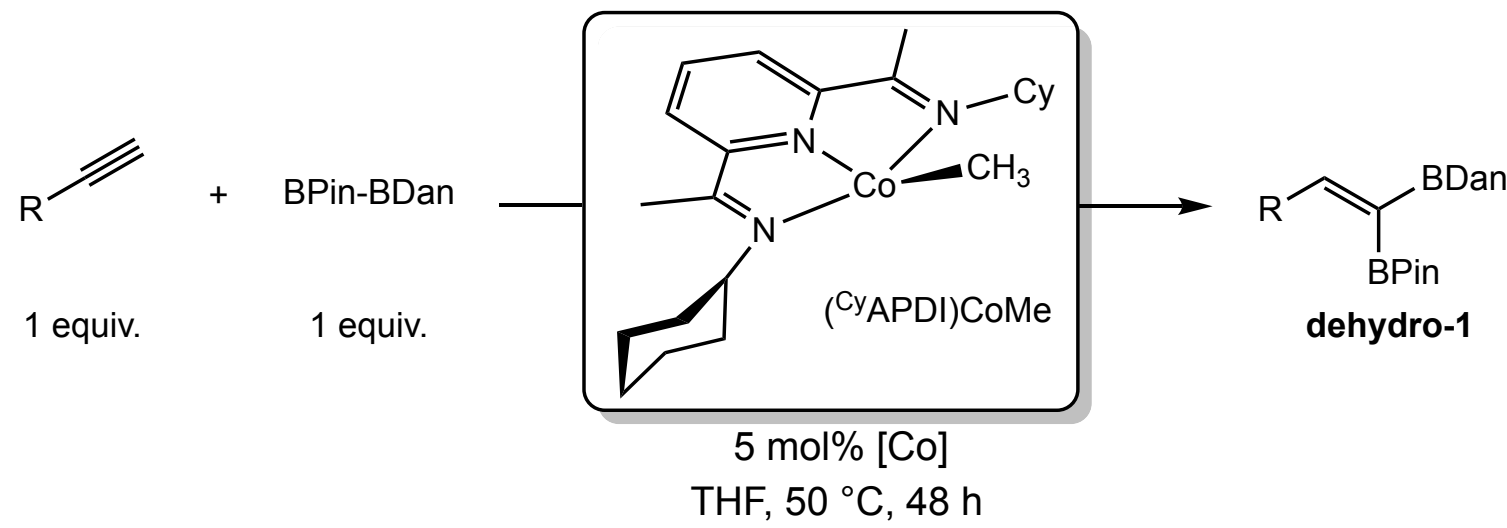

Reported 1,1-diboryl alkenes were prepared according to the general procedure by Chirik. ${ }^{7}$ Unreported compounds were prepared using the same procedure. In a nitrogen-filled glovebox, a $20 \mathrm{~mL}$ vial was charged with ( $\left.{ }^{\mathrm{Cy}} \mathrm{APDI}\right) \mathrm{CoMe}(0.03 \mathrm{mmol}, 12 \mathrm{mg}, 6 \mathrm{~mol} \%)$, THF $(0.5 \mathrm{~mL})$ and a Teflon-coated magnetic stir bar. Terminal alkyne $(0.5 \mathrm{mmol})$ was added in one portion, resulting in a color change from red to purple, and BPin-BDan (147 mg, $0.5 \mathrm{mmol}$ ) was added. The vial was sealed, taken out of the glovebox, and heated to $50{ }^{\circ} \mathrm{C}$ for $48 \mathrm{~h}$. The reaction was quenched by exposure to air, and filtered through a pad of silica gel, eluting with DCM. The solvent was removed, and purified using silica gel column chromatography, eluting with ethyl acetate:hexanes (1:50-1:20). 
(Z)-2-(1-(4,4,5,5-tetramethyl-1,3,2-dioxaborolan-2-yl)oct-1-en-1-yl)-2,3-dihydro-1H-naphtho[1,8de][1,3,2]diazaborinine (1b)<smiles>CCCCCCC=C(B1Nc2cccc3cccc(c23)N1)B1OC(C)(C)C(C)(C)O1</smiles>

Prepared according to general procedure A. $94 \mathrm{mg}, 48 \%$ isolated yield.

${ }^{1} \mathrm{H}$ NMR $(500 \mathrm{MHz}$, Chloroform- $d$ ) $\delta 7.01(\mathrm{t}, J=7.8 \mathrm{~Hz}, 2 \mathrm{H}), 6.89(\mathrm{~d}, J=8.2 \mathrm{~Hz}, 2 \mathrm{H}), 6.80(\mathrm{t}, J=$ $7.2 \mathrm{~Hz}, 1 \mathrm{H}), 6.23(\mathrm{~d}, J=7.3 \mathrm{~Hz}, 2 \mathrm{H}), 6.17(\mathrm{~s}, 2 \mathrm{H}), 2.41(\mathrm{q}, J=7.3 \mathrm{~Hz}, 2 \mathrm{H}), 1.41-1.31(\mathrm{~m}, 2 \mathrm{H})$, $1.25(\mathrm{~s}, 12 \mathrm{H}), 1.25-1.17(\mathrm{~m}, 4 \mathrm{H}), 0.86-0.75(\mathrm{~m}, 3 \mathrm{H})$.

${ }^{13} \mathrm{C}$ NMR $\left(126 \mathrm{MHz}, \mathrm{CDCl}_{3}\right) \delta 161.03,141.78,136.39,127.55,116.95,105.51,83.11,34.31$, $31.71,29.40,29.01,24.98,22.63,14.16$.

HRMS (ESI+): $m / z$ calculated for $\mathrm{C}_{24} \mathrm{H}_{37} \mathrm{~B}_{2} \mathrm{~N}_{2} \mathrm{O}_{2}{ }^{+}\left([\mathrm{MH}]^{+}\right)$: 407.3036 ; found 407.3030 
(Z)-2-(5-((tert-butyldimethylsilyl)oxy)-1-(4,4,5,5-tetramethyl-1,3,2-dioxaborolan-2-yl)pent-1-en-1yl)-2,3-dihydro-1 $H$-naphtho[1,8-de][1,3,2]diazaborinine (1c)<smiles>CC(C)(C)OCCC=C(B1Nc2cccc3cccc(c23)N1)B1OC(C)(C)C(C)(C)O1</smiles>

Prepared according to general procedure A. $190 \mathrm{mg}, 79 \%$ isolated yield.

${ }^{1} \mathrm{H}$ NMR (500 MHz, Chloroform-d) $\delta 7.09$ (dd, $\left.J=8.3,7.3 \mathrm{~Hz}, 2 \mathrm{H}\right), 6.97$ (dd, $J=8.3,0.8 \mathrm{~Hz}$, $2 \mathrm{H}), 6.93(\mathrm{t}, J=7.1 \mathrm{~Hz}, 1 \mathrm{H}), 6.30(\mathrm{dd}, J=7.3,1.0 \mathrm{~Hz}, 2 \mathrm{H}), 6.26(\mathrm{~s}, 2 \mathrm{H}), 3.74(\mathrm{t}, J=6.7 \mathrm{~Hz}, 2 \mathrm{H})$, $2.75(\mathrm{q}, J=6.8 \mathrm{~Hz}, 2 \mathrm{H}), 1.33(\mathrm{~s}, 12 \mathrm{H}), 0.92(\mathrm{~s}, 9 \mathrm{H}), 0.08(\mathrm{~s}, 6 \mathrm{H})$.

${ }^{13} \mathrm{C}$ NMR $\left(126 \mathrm{MHz} \mathrm{CDCl}_{3}\right) \delta 157.19,141.86,136.50,127.66,119.84,117.10,105.65,83.29$, $77.16,62.92,37.91,26.15,25.11,18.57,-5.01$.

HRMS (ESI+): $m / z$ calculated for $\mathrm{C}_{26} \mathrm{H}_{40} \mathrm{~B}_{2} \mathrm{~N}_{2} \mathrm{O}_{3} \mathrm{Si}^{+}([\mathrm{M}+]): 479.2994$, found: 479.2994. 
(S,Z)-2-(4,8-dimethyl-1-(4,4,5,5-tetramethyl-1,3,2-dioxaborolan-2-yl)nona-1,7-dien-1-yl)-2,3dihydro-1 $H$-naphtho[1,8-de][1,3,2]diazaborinine (19)<smiles>CC(C)=CCC[C@H](C)CC=C(B1Nc2cccc3cccc(c23)N1)B1OC(C)(C)C(C)(C)O1</smiles>

Prepared according to general procedure A. $88 \mathrm{mg}, 36 \%$ isolated yield.

${ }^{1} \mathrm{H}$ NMR (500 MHz, Chloroform-d) $\delta 7.26(\mathrm{~s}, 0 \mathrm{H}), 7.16-7.05(\mathrm{~m}, 2 \mathrm{H}), 7.01$ (t, $\left.J=8.3 \mathrm{~Hz}, \mathrm{OH}\right)$, $6.98(\mathrm{~d}, J=8.3 \mathrm{~Hz}, 1 \mathrm{H}), 6.90(\mathrm{t}, J=7.3 \mathrm{~Hz}, 1 \mathrm{H}), 6.32(\mathrm{~d}, J=7.3 \mathrm{~Hz}, 1 \mathrm{H}), 6.26(\mathrm{~s}, 2 \mathrm{H}), 5.13(\mathrm{tt}, J$ $=7.0,1.4 \mathrm{~Hz}, 1 \mathrm{H}), 2.53(\mathrm{ddd}, J=13.9,7.0,5.5 \mathrm{~Hz}, 1 \mathrm{H}), 2.37(\mathrm{dt}, J=13.9,7.8 \mathrm{~Hz}, 1 \mathrm{H}), 2.01$ (ddt, $J=22.1,14.1,8.1 \mathrm{~Hz}, 3 \mathrm{H}), 1.71(\mathrm{~s}, 2 \mathrm{H}), 1.63(\mathrm{~s}, 3 \mathrm{H}), 1.48-1.37(\mathrm{~m}, 1 \mathrm{H}), 1.34(\mathrm{~s}, 12 \mathrm{H})$, $1.26-1.14(\mathrm{~m}, 1 \mathrm{H}), 0.93(\mathrm{~d}, J=6.7 \mathrm{~Hz}, 3 \mathrm{H})$.

${ }^{13} \mathrm{C}$ NMR $(126 \mathrm{MHz}$, Chloroform-d) $\delta$ 159.68, 141.77, 136.41, 131.26, 127.56, 124.82, 119.71, 117.40, 116.98, 105.52, 83.13, 41.51, 37.12, 33.17, 25.78, 25.63, 25.01, 24.95, 19.60, 17.72.

HRMS (ESI+): $m / z$ calculated for $\mathrm{C}_{27} \mathrm{H}_{38} \mathrm{~B}_{2} \mathrm{~N}_{2} \mathrm{O}_{2}{ }^{+}\left([\mathrm{M}]^{+}\right): 444.3119$; found: 444.3117 . 
(Z)-2-(4-phenyl-1-(4,4,5,5-tetramethyl-1,3,2-dioxaborolan-2-yl)but-1-en-1-yl)-2,3-dihydro-1Hnaphtho[1,8-de][1,3,2]diazaborinine $(\mathbf{1 h})$<smiles></smiles>

Prepared according to general procedure A. $148 \mathrm{mg}, 70 \%$ isolated yield.

${ }^{1} \mathrm{H}$ NMR $(500 \mathrm{MHz}$, Chloroform-d) $\delta 7.29(\mathrm{td}, J=7.4,1.4 \mathrm{~Hz}, 2 \mathrm{H}), 7.24-7.17(\mathrm{~m}, 3 \mathrm{H}), 7.08$ (dd, $J=8.3,7.3 \mathrm{~Hz}, 2 \mathrm{H}), 6.96(\mathrm{dd}, J=8.3,0.9 \mathrm{~Hz}, 2 \mathrm{H}), 6.93(\mathrm{t}, J=6.8 \mathrm{~Hz}, 1 \mathrm{H}), 6.29(\mathrm{dd}, J=7.3,1.0$ $\mathrm{Hz}, 2 \mathrm{H}), 6.24(\mathrm{~s}, 2 \mathrm{H}), 2.85-2.73(\mathrm{~m}, 4 \mathrm{H}), 1.32(\mathrm{~s}, 12 \mathrm{H})$.

${ }^{13} \mathrm{C} \mathrm{NMR}\left(126 \mathrm{MHz}, \mathrm{CDCl}_{3}\right) \delta 159.54,141.71,136.38,128.49,128.37,127.54,125.89,122.78$, $117.01,105.53,83.17,36.04,35.90,25.00,22.46$.

HRMS (ESI+): $m / z$ calculated for $\mathrm{C}_{26} \mathrm{H}_{31} \mathrm{~B}_{2} \mathrm{~N}_{2} \mathrm{O}_{2}{ }^{+}\left([\mathrm{MH}]^{+}\right)$: 425.2566 ; found: 425.2575 
(Z)-2-(3-phenyl-1-(4,4,5,5-tetramethyl-1,3,2-dioxaborolan-2-yl)prop-1-en-1-yl)-2,3-dihydro-1Hnaphtho[1,8-de][1,3,2]diazaborinine (1i)<smiles>CC1(C)OB(C(=CCc2ccccc2)B2Nc3cccc4cccc(c34)N2)OC1(C)C</smiles>

Prepared according to general procedure A. $120 \mathrm{mg}, 59 \%$ isolated yield.

${ }^{1} \mathrm{H}$ NMR $(500 \mathrm{MHz}$, Chloroform- $d) \delta 7.33(\mathrm{t}, J=7.6 \mathrm{~Hz}, 2 \mathrm{H}), 7.29-7.25(\mathrm{~m}, 2 \mathrm{H}), 7.25-7.20$ (m, 1H), 7.07 (dd, $J=8.3,7.3 \mathrm{~Hz}, 2 \mathrm{H}), 6.96(\mathrm{dd}, J=8.4,1.0 \mathrm{~Hz}, 2 \mathrm{H}), 6.93$ (t (overlapping), $J=$ $7.2 \mathrm{~Hz}, 1 \mathrm{H}), 6.28(\mathrm{dd}, J=7.4,1.1 \mathrm{~Hz}, 2 \mathrm{H}), 6.26(\mathrm{~s}, 2 \mathrm{H}), 3.86(\mathrm{~d}, J=7.2 \mathrm{~Hz}, 2 \mathrm{H}), 1.37(\mathrm{~s}, 12 \mathrm{H})$.

${ }^{13} \mathrm{C}$ NMR $\left(126 \mathrm{MHz}, \mathrm{CDCl}_{3}\right) \delta 161.03,141.78,136.39,127.55,116.95,105.51,83.11,34.31$, $31.71,29.40,29.01,24.98,22.63,14.16$.

HRMS (ESI+): $\mathrm{m} / \mathrm{z}$ calculated for $\mathrm{C}_{25} \mathrm{H}_{29} \mathrm{~B}_{2} \mathrm{~N}_{2} \mathrm{O}_{2}{ }^{+}\left([\mathrm{MH}]^{+}\right): 411.2410$; found: 411.2410 
(Z)-2-(2-phenyl-1-(4,4,5,5-tetramethyl-1,3,2-dioxaborolan-2-yl)vinyl)-2,3-dihydro-1Hnaphtho[1,8-de][1,3,2]diazaborinine (1j)<smiles></smiles>

Prepared according to a slightly modified general procedure A. A $20 \mathrm{~mL}$ vial was charged with ( $\left.{ }^{\mathrm{Cy}} \mathrm{APDI}\right) \mathrm{CoMe}(0.05 \mathrm{mmol}, 24 \mathrm{mg}, 10 \mathrm{~mol} \%)$, THF $(0.5 \mathrm{~mL})$ and a Teflon-coated magnetic stir bar. Phenylacetylene ( $55 \mu \mathrm{l}, 0.5 \mathrm{mmol}$ ) was added in one portion, resulting in a color change from red to purple, and BPin-BDan (147 mg, $0.5 \mathrm{mmol}$ ) was added. The vial was sealed, taken out of the glovebox, and heated to $50^{\circ} \mathrm{C}$ for $24 \mathrm{~h}$. The vial was then brought back into the glovebox, and ( $\left.{ }^{\mathrm{Cy}} \mathrm{APDI}\right) \mathrm{CoMe}(0.05 \mathrm{mmol}, 24 \mathrm{mg}, 10 \mathrm{~mol} \%)$, and phenylacetylene (55 $\mu \mathrm{l}, 0.5$ mmol) was again added in one portion. The vial was sealed, taken out of the glovebox, and heated to $50^{\circ} \mathrm{C}$ for $24 \mathrm{~h}$. This was repeated one more time for a total of 3 additions of alkyne and catalyst. The reaction was quenched by exposure to air, and filtered through a pad of silica gel, eluting with ethyl acetate/hexanes. The solvent was removed, and purified using silica gel column chromatography, eluting with ethyl acetate:hexanes (1:10). $31 \mathrm{mg}, 15 \%$ isolated yield.

${ }^{1} \mathrm{H}$ NMR $(500 \mathrm{MHz}$, Chloroform-d) $\delta 7.49(\mathrm{~s}, 1 \mathrm{H}), 7.43-7.36(\mathrm{~m}, 2 \mathrm{H}), 7.30-7.23(\mathrm{~m}, 3 \mathrm{H}), 7.04$ $(\mathrm{dd}, J=8.3,7.3 \mathrm{~Hz}, 2 \mathrm{H}), 6.94(\mathrm{dd}, J=8.3,0.9 \mathrm{~Hz}, 2 \mathrm{H}), 6.28(\mathrm{dd}, J=7.4,1.0 \mathrm{~Hz}, 2 \mathrm{H}), 6.11(\mathrm{~d}, J$ $=3.3 \mathrm{~Hz}, 2 \mathrm{H}), 1.27(\mathrm{~s}, 12 \mathrm{H})$.

${ }^{13} \mathrm{C} \mathrm{NMR}\left(126 \mathrm{MHz}, \mathrm{CDCl}_{3}\right) \delta 156.52,151.25,141.44,139.36,136.39,128.47,128.35,128.15$, 127.60, 117.41, 105.78, 83.89, 24.91.

HRMS (ESI+): $m / z$ calculated for $\mathrm{C}_{24} \mathrm{H}_{27} \mathrm{~B}_{2} \mathrm{~N}_{2} \mathrm{O}_{2}{ }^{+}\left([\mathrm{MH}]^{+}\right)$: 397.2253; found: 397.2262. 
(Z)-2-(2-cyclopentyl-1-(4,4,5,5-tetramethyl-1,3,2-dioxaborolan-2-yl)vinyl)-2,3-dihydro-1Hnaphtho[1,8-de][1,3,2]diazaborinine (1k)<smiles>CC1(C)OB(/C(=C\C2CCCC2)B2Nc3cccc4cccc(c34)N2)OC1(C)C</smiles>

Prepared according to a slightly modified general procedure $\mathbf{A}$ using $10 \mathrm{~mol} \%\left({ }^{\mathrm{C}} \mathrm{APDI}\right) \mathrm{CoMe}$. $159 \mathrm{mg}, 80 \%$.

${ }^{1} \mathrm{H}$ NMR $(500 \mathrm{MHz}$, Chloroform-d) $\delta 7.09$ (dd, $J=8.3,7.4 \mathrm{~Hz}, 2 \mathrm{H}), 6.98(\mathrm{dd}, J=8.3,1.0 \mathrm{~Hz}$, $2 \mathrm{H}), 6.74(\mathrm{~d}, J=9.1 \mathrm{~Hz}, 1 \mathrm{H}), 6.31(\mathrm{dd}, J=7.3,1.0 \mathrm{~Hz}, 2 \mathrm{H}), 6.24(\mathrm{~s}, 2 \mathrm{H}), 3.20(\mathrm{dt}, J=8.8,7.6$ $\mathrm{Hz}, 1 \mathrm{H}), 1.90-1.85(\mathrm{~m}, 2 \mathrm{H}), 1.77-1.68(\mathrm{~m}, 2 \mathrm{H}), 1.68-1.58(\mathrm{~m}, 2 \mathrm{H}), 1.49-1.34(\mathrm{~m}, 2 \mathrm{H})$, $1.34(\mathrm{~s}, 12 \mathrm{H})$.

${ }^{13} \mathrm{C}$ NMR $\left(126 \mathrm{MHz}, \mathrm{CDCl}_{3}\right) \delta 165.64,141.79,136.39,127.55,119.68,116.93,105.49,83.10$, 44.44, 33.90, 25.89, 24.95.

HRMS (ESI+): $\mathrm{m} / \mathrm{z}$ calculated $\mathrm{C}_{23} \mathrm{H}_{30} \mathrm{~B}_{2} \mathrm{~N}_{2} \mathrm{O}_{2}{ }^{+}\left([\mathrm{M}]^{+}\right): 388.2493$; found: 388.2501 . 
(Z)-2-(2-cyclohexyl-1-(4,4,5,5-tetramethyl-1,3,2-dioxaborolan-2-yl)vinyl)-2,3-dihydro-1Hnaphtho[1,8-de][1,3,2]diazaborinine (1I)<smiles>CC1(C)OB(C(=CC2CCCCC2)B2Nc3cccc4cccc(c34)N2)OC1(C)C</smiles>

Prepared according to a slightly modified general procedure $\mathbf{A}$ using $10 \mathrm{~mol} \%\left({ }^{\mathrm{C}} \mathrm{APDI}\right) \mathrm{CoMe}$. $121 \mathrm{mg}, 61 \%$.

${ }^{1} \mathrm{H}$ NMR $(500 \mathrm{MHz}$, Chloroform-d) $\delta 7.00$ (dd, $J=8.3,7.3 \mathrm{~Hz}, 2 \mathrm{H}), 6.89$ (dd, $J=8.3,1.0 \mathrm{~Hz}$, $2 \mathrm{H}), 6.58(\mathrm{~d}, J=8.8 \mathrm{~Hz}, 1 \mathrm{H}), 6.22(\mathrm{dd}, J=7.3,1.0 \mathrm{~Hz}, 2 \mathrm{H}), 6.13(\mathrm{~s}, 2 \mathrm{H}), 2.65$ (tdd, $J=11.2$, 8.8, $5.6 \mathrm{~Hz}, 1 \mathrm{H}), 1.77-1.55(\mathrm{~m}, 5 \mathrm{H}), 1.25(\mathrm{~s}, 12 \mathrm{H}), 1.22-1.19(\mathrm{~m}, 2 \mathrm{H}), 1.14-1.01(\mathrm{~m}, 3 \mathrm{H})$.

${ }^{13} \mathrm{C}$ NMR $\left(126 \mathrm{MHz}, \mathrm{CDCl}_{3}\right) \delta 165.78,141.76,136.39,127.55,116.96,105.50,83.12,42.60$, $33.16,25.98,25.81,24.94$.

HRMS (ESI+): $m / z$ calculated $\mathrm{C}_{24} \mathrm{H}_{32} \mathrm{~B}_{2} \mathrm{~N}_{2} \mathrm{O}_{2}{ }^{+}\left([\mathrm{M}]^{+}\right): 402.2650$; found: 402.2643 
(Z)-2,2'-(1-(4,4,5,5-tetramethyl-1,3,2-dioxaborolan-2-yl)ethene-1,2-diyl)bis(2,3-dihydro-1Hnaphtho[1,8-de][1,3,2]diazaborinine) $(1 \mathrm{~m})$<smiles>CC1(C)OB(/C(=C\B2Nc3cccc4cccc(c34)N2)B2Nc3cccc4cccc(c34)N2)OC1(C)C</smiles>

Prepared according to a slightly modified general procedure A using $20 \mathrm{~mol} \%\left({ }^{\mathrm{C}} \mathrm{APDI}\right) \mathrm{CoMe}$, and stirring for 72 h. $50 \mathrm{mg}, 41 \%$.

${ }^{1} \mathrm{H}$ NMR $(500 \mathrm{MHz}$, Chloroform- $d$ ) $\delta 7.04$ (ddd, $J=8.3,7.3,3.7 \mathrm{~Hz}, 4 \mathrm{H}), 6.98-6.90(\mathrm{~m}, 5 \mathrm{H})$, $6.66(\mathrm{~s}, 2 \mathrm{H}), 6.27$ (dd, J = 7.2, $0.9 \mathrm{~Hz}, 2 \mathrm{H}), 6.23(\mathrm{dd}, J=7.3,1.0 \mathrm{~Hz}, 2 \mathrm{H}), 6.22(\mathrm{~s}, 2 \mathrm{H}), 1.35$ (s, $12 \mathrm{H})$.

${ }^{13} \mathrm{C} \mathrm{NMR}\left(126 \mathrm{MHz}, \mathrm{CDCl}_{3}\right) \delta 141.54,141.47,136.49,136.41,127.66,127.61,120.24,119.93$, $117.45,117.39,105.81,105.67,84.25,29.73,25.08$.

HRMS (ESI+): $m / z$ calculated $\mathrm{C}_{28} \mathrm{H}_{29} \mathrm{~B}_{3} \mathrm{~N}_{4} \mathrm{O}_{2}{ }^{+}\left([\mathrm{M}]^{+}\right): 486.2570$; found: 486.2574 
Preparation of Indenyl and Styrenyl Boronates:
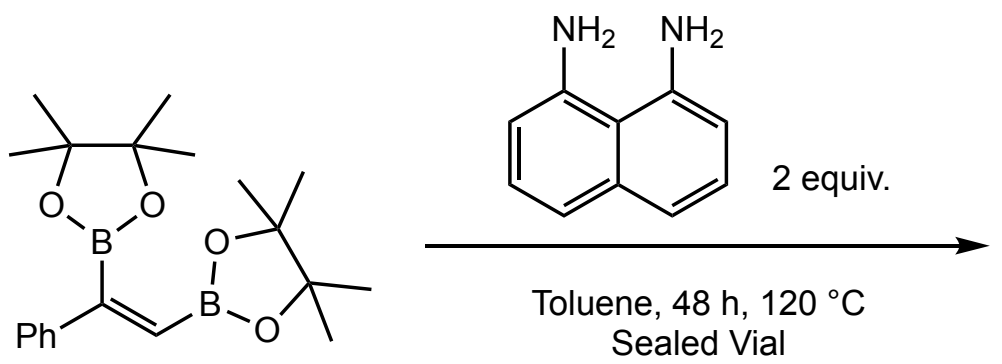<smiles>P/C=C\C(=Cc1ccccc1)B1Nc2cccc3cccc(c23)N1</smiles>

(E)-2,2'-(1-phenylethene-1,2-diyl)bis(2,3-dihydro-1H-naphtho[1,8-de][1,3,2]diazaborinine) (3d): In a $20 \mathrm{~mL}$ vial equipped with a teflon-coated magnetic stir bar was added (E)-2,2'-(1phenylethene-1,2-diyl)bis(4,4,5,5-tetramethyl-1,3,2-dioxaborolane) (200 mg, $0.56 \mathrm{mmol}, 1$ equiv.) and diaminonapthalene (178 mg, $1.12 \mathrm{mmol}, 2$ equiv.). Toluene (1 mL) was added, and the vial heated to $120^{\circ} \mathrm{C}$ for $48 \mathrm{~h}$. The vial was allowed to cool, and directly loaded onto a silica gel column, eluting with hexanes: ethyl acetate $20: 1$ to furnish $(E)-2,2$ '-(1-phenylethene-1,2diyl)bis(2,3-dihydro-1H-naphtho[1,8-de][1,3,2]diazaborinine). Off-white solid. $50 \mathrm{mg}, 11 \%$ yield.

${ }^{1} \mathrm{H}$ NMR $(500 \mathrm{MHz}$, Chloroform-d) $\delta 7.43(\mathrm{~d}, J=7.5 \mathrm{~Hz}, 2 \mathrm{H}), 7.27$ (dt, $J=27.6,7.2 \mathrm{~Hz}, 3 \mathrm{H})$, $7.12-7.00(\mathrm{~m}, 4 \mathrm{H}), 7.00-6.79(\mathrm{~m}, 4 \mathrm{H}), 6.41(\mathrm{~s}, 1 \mathrm{H}), 6.27(\mathrm{~d}, J=6.9 \mathrm{~Hz}, 2 \mathrm{H}), 6.08(\mathrm{~d}, J=7.1$ $\mathrm{Hz}, 2 \mathrm{H}), 5.97(\mathrm{~s}, 2 \mathrm{H}), 5.81(\mathrm{~s}, 2 \mathrm{H})$.

${ }^{13} \mathrm{C} \mathrm{NMR}\left(126 \mathrm{MHz}, \mathrm{CDCl}_{3}\right) \delta 143.22,140.92,140.16,136.32,136.24,128.73,128.04,127.72$, $127.62,127.00,119.82,119.74,118.44,117.68,106.47,106.00$. 
<smiles>OB(O)C1=CCc2ccccc21</smiles>

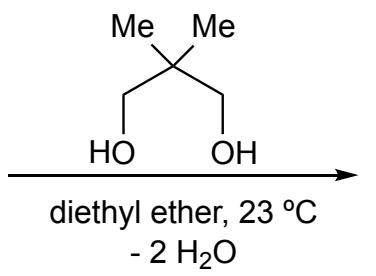<smiles>COC(=O)C1=CCc2ccccc21</smiles><smiles>CC1(C)COBOC1</smiles>

2-(1H-inden-3-yl)-5,5-dimethyl-1,3,2-dioxaborolane (5c). Prepared according to a modified procedure previously reported by Crudden and coworkers. ${ }^{8}$ An oven-dried $50 \mathrm{~mL}$ round bottom flask was charged with ( $1 H$-inden-3-yl)boronate acid $(0.300 \mathrm{~g}, 1.875 \mathrm{mmol})$, a magnetic stir bar, $20 \mathrm{~mL}$ diethyl ether, and anhydrous magnesium sulfate $(0.226 \mathrm{~g}, 1.875 \mathrm{mmol})$. While stirring the reaction, 2,2-dimethylpropane-2,3-diol $(0.195 \mathrm{~g}, 1.875 \mathrm{mmol})$ was added and the reaction was allowed to continue to stir at $23^{\circ} \mathrm{C}$ with monitoring by TLC until the reaction was complete. The reaction was then directly filtered by vacuum filtration through celite on a fritted-glass filter and washed with diethyl ether $(50 \mathrm{~mL})$. The combined organic layers were concentrated in vacuo to afford the desired product in quantitative yield $(0.428 \mathrm{~g}, 1.876 \mathrm{mmol})$. The product was recrystallized from pentane at $-35^{\circ} \mathrm{C}$ prior to use in catalysis.

${ }^{1} \mathrm{H}$ NMR $\left(500 \mathrm{MHz}, \mathrm{CDCl}_{3}, 23^{\circ} \mathrm{C}\right): \delta 7.87(\mathrm{~d}, J=7.6 \mathrm{~Hz}, 1 \mathrm{H}$, indenyl $\mathrm{CH}), 7.46(\mathrm{~d}, J=7.4 \mathrm{~Hz}, 1 \mathrm{H}$, indenyl $\mathrm{CH}$ ), $7.27(\mathrm{t}, \mathrm{J}=7.5 \mathrm{~Hz}, 1 \mathrm{H}$, indenyl $\mathrm{CH}$ ), 7.17 (obsc. $\mathrm{m}, 2 \mathrm{H}, 2$-indenyl $\mathrm{CH}$ ), $3.79(\mathrm{~s}, 4 \mathrm{H}$, BNeop $\mathrm{CH}_{2}$ ), 3.43 (s, 2H, 1-indenyl $\mathrm{CH}_{2}$ ), 1.04 (s, 6H, BNeop $\mathrm{CH}_{3}$ ).

${ }^{13} \mathrm{C}$ NMR $\left(126 \mathrm{MHz}, \mathrm{CDCl}_{3}, 23{ }^{\circ} \mathrm{C}\right): \delta 147.5,147.2,144.4,126.2,124.3,123.5,122.9,72.3,40.4$, 22.1 .

HRMS (ESI+) $\mathrm{m} / \mathrm{z}$ calculated for $\mathrm{C}_{14} \mathrm{H}_{17} \mathrm{BO}_{2}{ }^{+}\left(\mathrm{M}^{+}\right)$228.1322, found $\mathrm{m} / \mathrm{z} 228.1320$. 

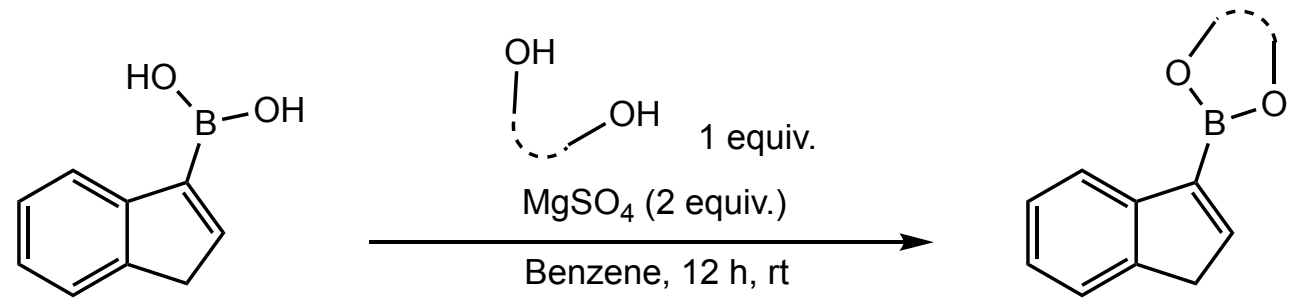

To an oven dried Schlenk flask equipped with a Teflon coated stir bar was added 1-H-Inden-3yl boronic acid (320 mg, 2 mmol, 1 equiv.), $\mathrm{MgSO}_{4}$ ( $481 \mathrm{mg}, 4 \mathrm{mmol}, 2$ equiv.), and diol (2 mmol, 1 equiv.). Anhydrous benzene ( $2 \mathrm{~mL})$ was added under argon, and the mixture stirred for $12 \mathrm{~h}$. After $12 \mathrm{~h}$, the solvent was removed. Benzene $(2 \mathrm{~mL})$ was added, and removed. This trituration process was repeated 3 times. The flask containing dry solid was brought into a nitrogen filled glovebox, dissolved in benzene and filtered through celite. The solvent was removed, and benzene $(\sim 1 \mathrm{~mL})$ was added and removed 5 times. 
2-(1H-inden-3-yl)-4,4,5,5-tetramethyl-1,3,2-dioxaborolane (5d):

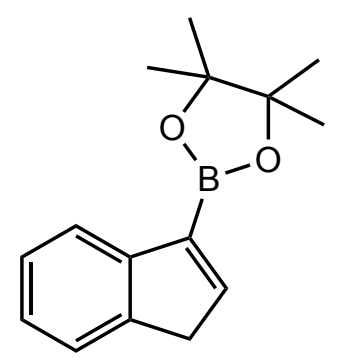

White solid. $290 \mathrm{mg}, 30 \%$.

${ }^{1} \mathrm{H}$ NMR $(500 \mathrm{MHz}$, Chloroform-d) $\delta 7.85(\mathrm{~d}, J=7.5 \mathrm{~Hz}, 1 \mathrm{H}), 7.47(\mathrm{~d}, J=7.2 \mathrm{~Hz}, 1 \mathrm{H}), 7.30(\mathrm{t}, J$ $=7.4 \mathrm{~Hz}, 2 \mathrm{H}), 7.19(\mathrm{t}, J=7.3 \mathrm{~Hz}, 1 \mathrm{H}), 3.47(\mathrm{~s}, 2 \mathrm{H}), 1.36(\mathrm{~s}, 12 \mathrm{H})$.

${ }^{13} \mathrm{C}$ NMR $\left(126 \mathrm{MHz}, \mathrm{CDCl}_{3}\right) \delta 149.15,146.94,143.96,126.33,124.60,123.55,122.88,83.50$, 40.76, 25.06.

HRMS (ESI+) $\mathrm{m} / \mathrm{z}$ calculated for $\mathrm{C}_{15} \mathrm{H}_{20} \mathrm{BO}_{2}{ }^{+}\left(\mathrm{MH}^{+}\right): 243.1551$, found: 243.1543 . 
2-(1H-inden-3-yl)-1,3,2-dioxaborolane (5f):

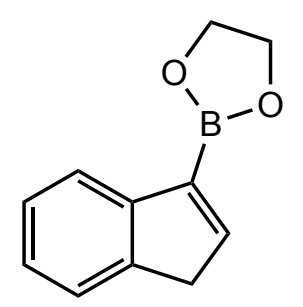

Yellow solid. 2 mmol scale. 150 mg, $40 \%$. Poor moisture stability prevented HRMS analysis.

${ }^{1} \mathrm{H}$ NMR $\left(500 \mathrm{MHz}\right.$, Benzene- $\left.d_{6}\right) \delta 8.26(\mathrm{~d}, J=7.6 \mathrm{~Hz}, 1 \mathrm{H}), 7.38-7.24(\mathrm{~m}, 3 \mathrm{H}), 7.16(\mathrm{t}, J=5.6$ $\mathrm{Hz}, 1 \mathrm{H}), 3.63(\mathrm{~s}, 4 \mathrm{H}), 3.12(\mathrm{~s}, 2 \mathrm{H})$.

${ }^{13} \mathrm{C}$ NMR $\left(126 \mathrm{MHz}\right.$, Benzene- $\left.d_{6}\right) \delta 149.76,146.86,143.90,126.39,124.71,123.51,122.96$, $65.13,40.46$. 
2-(1H-inden-3-yl)benzo[d][1,3,2]dioxaborole $(5 \mathbf{g})$ :<smiles>C1=C(B2Oc3ccccc3O2)c2ccccc2C1</smiles>

Off white-solid. $100 \mathrm{mg}, 21 \%$. Poor moisture stability prevented HRMS analysis.

${ }^{1} \mathrm{H}$ NMR $\left(500 \mathrm{MHz}\right.$, Benzene- $\left.d_{6}\right) \delta 8.30(\mathrm{~d}, J=7.6 \mathrm{~Hz}, 1 \mathrm{H}), 7.38(\mathrm{t}, J=2.0 \mathrm{~Hz}, 1 \mathrm{H}), 7.35$ (dd, $J$ $=7.4,1.0 \mathrm{~Hz}, 1 \mathrm{H}), 7.31(\mathrm{~d}, J=7.4 \mathrm{~Hz}, 1 \mathrm{H}), 7.19(\mathrm{dd}, J=7.4,1.1 \mathrm{~Hz}, 1 \mathrm{H}), 7.17(\mathrm{dd}, J=5.1,1.6$ $\mathrm{Hz}, 1 \mathrm{H}), 7.11(\mathrm{dd}, J=5.8,3.4 \mathrm{~Hz}, 2 \mathrm{H}), 6.83(\mathrm{dd}, J=5.9,3.3 \mathrm{~Hz}, 2 \mathrm{H}), 3.09(\mathrm{~d}, J=1.9 \mathrm{~Hz}, 2 \mathrm{H})$.

${ }^{13} \mathrm{C}$ NMR $\left(126 \mathrm{MHz}, \mathrm{C}_{6} \mathrm{D}_{6}\right) \delta 151.65,148.27,145.78,143.67,126.55,125.08,123.59,122.81$, $122.67,112.47,40.74$. 

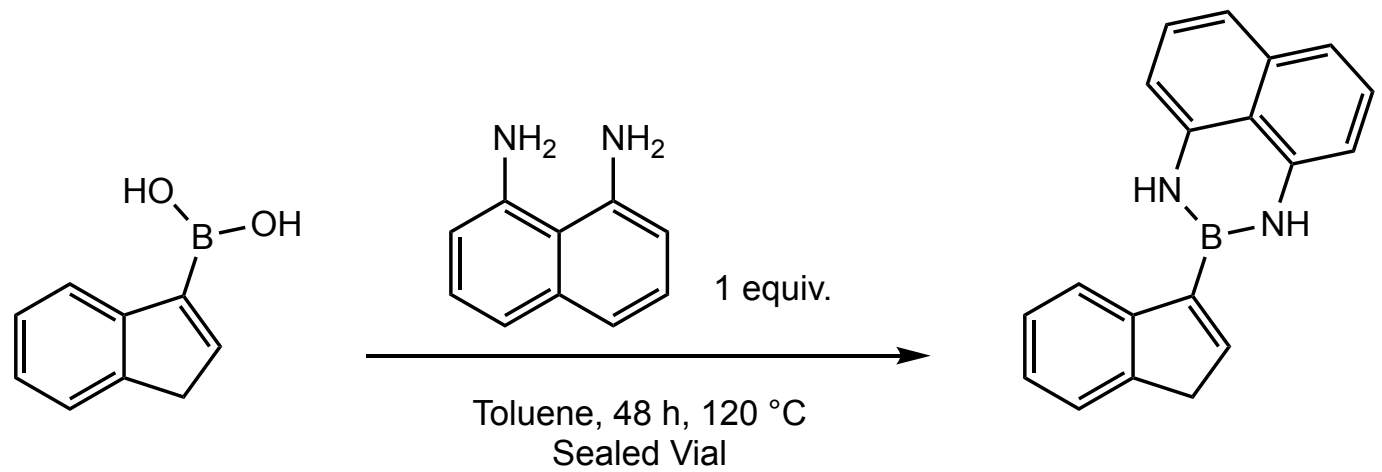

(E)-2,2'-(1-phenylethene-1,2-diyl)bis(2,3-dihydro-1H-naphtho[1,8-de][1,3,2]diazaborinine) (5e):

In a $20 \mathrm{~mL}$ vial equipped with a teflon-coated magnetic stir bar was added 1-H-Inden-3-yl

boronic acid (640 mg, 4 mmol, 1 equiv.) and diaminonapthalene (633 mg, 4 mmol, 4 equiv.).

Toluene $\left(2 \mathrm{~mL}\right.$ ) was added, and the vial heated to $120^{\circ} \mathrm{C}$ for $48 \mathrm{~h}$. The vial was allowed to cool, and directly loaded onto a silica gel column, eluting with hexanes: ethyl acetate 20:1 to furnish

(E)-2,2'-(1-phenylethene-1,2-diyl)bis(2,3-dihydro-1H-naphtho[1,8-de][1,3,2]diazaborinine).

Off-white solid. $210 \mathrm{mg}, 19 \%$ yield.

${ }^{1} \mathrm{H}$ NMR $(500 \mathrm{MHz}$, Chloroform- $d) \delta 7.65(\mathrm{~d}, J=7.3 \mathrm{~Hz}, 1 \mathrm{H}), 7.58(\mathrm{~d}, J=7.1 \mathrm{~Hz}, 1 \mathrm{H}), 7.38(\mathrm{t}, J$ $=7.2 \mathrm{~Hz}, 1 \mathrm{H}), 7.29(\mathrm{~d}, J=6.4 \mathrm{~Hz}, 2 \mathrm{H}), 7.18(\mathrm{t}, J=7.6 \mathrm{~Hz}, 2 \mathrm{H}), 7.10(\mathrm{~d}, J=8.2 \mathrm{~Hz}, 2 \mathrm{H}), 7.00(\mathrm{~s}$, $1 \mathrm{H}), 6.43(\mathrm{~d}, J=7.3 \mathrm{~Hz}, 2 \mathrm{H}), 6.04(\mathrm{~s}, 2 \mathrm{H}), 3.53(\mathrm{~s}, 2 \mathrm{H})$.

${ }^{13} \mathrm{C}$ NMR $\left(126 \mathrm{MHz}, \mathrm{CDCl}_{3}\right) \delta 146.70,144.49,142.79,141.03,136.42,127.64,126.43,124.83$, $124.11,121.28,120.05,117.88,106.02,40.47$.

HRMS (ESI+) $\mathrm{m} / z$ calculated for $\mathrm{C}_{19} \mathrm{H}_{15} \mathrm{BN}_{2}{ }^{+}\left(\mathrm{M}^{+}\right) 282.1328$, found: 282.1325 . 


\section{General Hydrogenation Procedure and 1,1-Diboryl Alkanes}
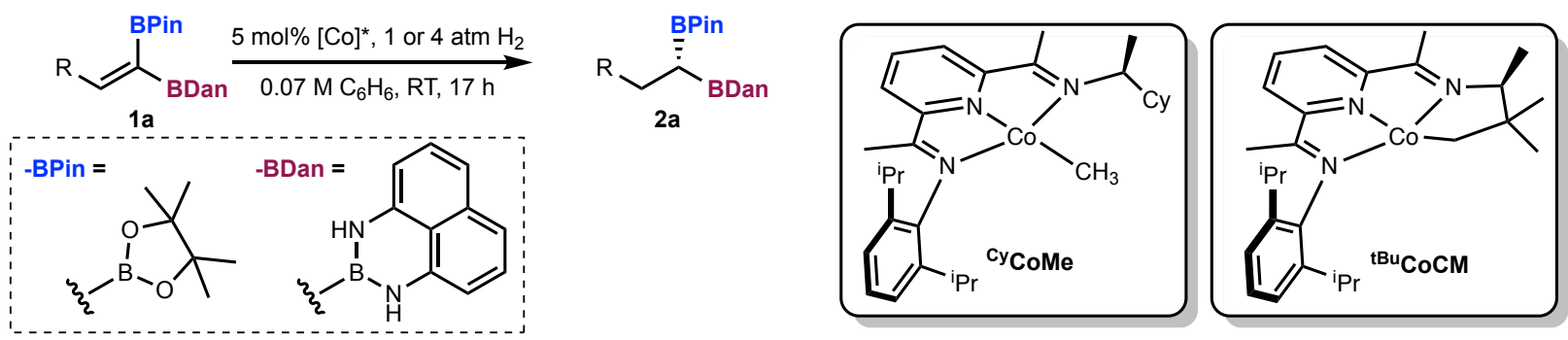

General Procedure B - Asymmetric Hydrogenation of 1,1-diboryl alkenes: To a $20 \mathrm{~mL}$ vial containing cobalt complex ${ }^{\mathrm{tBu}} \mathrm{CoCM}$ was added 1,1-diborylalkene substrate 1 (0.10 mmol) and benzene $(1.0 \mathrm{~mL})$. The solution was transferred to a thick walled glass bomb with a glass pipette, and the vial was washed with benzene $(1 \times 0.5 \mathrm{~mL})$, so that the total volume of the reaction solution was $1.5 \mathrm{~mL}$. A magnetic stir bar was added, and the bomb was sealed with a Kontes valve. The vessel was brought out of the glovebox and connected to a high vacuum line. The vessel was frozen in liquid nitrogen, evacuated, and backfilled with $1 \mathrm{~atm}$ of hydrogen gas at liquid nitrogen temperature, corresponding to approximately $4 \mathrm{~atm}$ of hydrogen pressure at $23^{\circ} \mathrm{C}$. The frozen reaction solution was then left to thaw, after which it was magnetically stirred for the period of time indicated. The color of the reaction solution under an atmosphere of hydrogen was purple (the same shade of purple as when cobalt complex ${ }^{\mathrm{tBu}} \mathrm{CoCM}$ is dissolved in benzene). The vessel was then opened to air, and the mixture was filtered through a plug of silica with hexane/EtOAc. $A{ }^{1} \mathrm{H}$ NMR spectrum of the unpurified reaction mixture was recorded to determine if the reaction had gone to completion. If necessary, purification of the mixture by flash chromatography on silica gel using a mixture of hexane and ethyl acetate as eluent furnished the 1,1-diborylalkane product. The initial screening of conditions used this procedure exactly, by varying the choice of cobalt complex and hydrogen pressure. Racemic compounds were prepared by $\mathrm{Pd} / \mathrm{C}$ catalyzed hydrogenation of the corresponding alkenes. 
Gram-Scale Hydrogenation of 1a: To a $20 \mathrm{~mL}$ vial containing cobalt complex ${ }^{\mathrm{tBu}} \mathrm{CoCM}$ (12 mg, $0.0256 \mathrm{mmol}, 0.01$ equiv.) was added 1,1-diborylalkene substrate $1 \mathrm{a}(1.00 \mathrm{~g}, 2.56 \mathrm{mmol}, 1$ equiv.) and benzene $(10 \mathrm{~mL})$. The solution was transferred to a thick walled glass bomb with a glass pipette, and the vial was washed with benzene $(2 \times 5 \mathrm{~mL})$, so that the total volume of the reaction solution was $20 \mathrm{~mL}$. A large magnetic stir bar was added, and the bomb was sealed with a Kontes valve. The vessel was brought out of the glovebox and connected to a high vacuum line. The vessel was frozen in liquid nitrogen, evacuated, and backfilled with $1 \mathrm{~atm}$ of hydrogen gas at liquid nitrogen temperature, corresponding to approximately $4 \mathrm{~atm}$ of hydrogen pressure at $23^{\circ} \mathrm{C}$. The frozen reaction solution was then left to thaw, after which it was stirred vigorously for $36 \mathrm{~h}$. The vessel was opened to air, and filtered through a pad of silica gel, eluting with DCM. The solvent was removed affording 2 a as a white solid. $954 \mathrm{mg}, 95 \%$ yield, $95 \%$ ee. 
(S)-2-(1-(4,4,5,5-tetramethyl-1,3,2-dioxaborolan-2-yl)heptyl)-2,3-dihydro-1H-naphtho[1,8de][1,3,2]diazaborinine $(\mathbf{2 a})$<smiles>CCCCCCC(B1Nc2cccc3cccc(c23)N1)B1OC(C)(C)C(C)(C)O1</smiles>

Prepared according to general procedure B. White crystalline solid. $40 \mathrm{mg}, 98 \%$ isolated yield.

${ }^{1} \mathrm{H}$ NMR $(500 \mathrm{MHz}$, Chloroform-d) $\delta 7.09(\mathrm{t}, J=7.8 \mathrm{~Hz}, 2 \mathrm{H}), 6.99(\mathrm{~d}, J=8.2 \mathrm{~Hz}, 2 \mathrm{H}), 6.29(\mathrm{~d}, J$ $=7.3 \mathrm{~Hz}, 2 \mathrm{H}), 5.78(\mathrm{~s}, 2 \mathrm{H}), 1.69-1.57(\mathrm{~m}, 1 \mathrm{H}), 1.51(\mathrm{dtd}, J=12.6,9.1,7.7,4.2 \mathrm{~Hz}, 1 \mathrm{H}), 1.41-$ $1.26(\mathrm{~m}, 6 \mathrm{H}), 1.25(\mathrm{~s}, 6 \mathrm{H}), 1.24(\mathrm{~s}, 6 \mathrm{H}), 1.16(\mathrm{dd}, J=6.9,4.1 \mathrm{~Hz}, 1 \mathrm{H}), 0.89(\mathrm{t}, J=6.7 \mathrm{~Hz}, 3 \mathrm{H})$.

${ }^{13} \mathrm{C} \mathrm{NMR}\left(126 \mathrm{MHz}, \mathrm{CDCl}_{3}\right) \delta 141.38,136.30,127.56,119.50,117.20,105.42,83.14,32.38$, $31.79,29.42,26.44,25.04,24.51,22.67,14.13$.

HRMS (ESI+): $m / z$ calculated $\mathrm{C}_{23} \mathrm{H}_{34} \mathrm{~B}_{2} \mathrm{~N}_{2} \mathrm{O}_{2}{ }^{+}\left([\mathrm{M}]^{+}\right)$: 392.2806; found: 392.2809 .

HPLC (Chiralcel OJ-H): $40 \% \mathrm{MeOH}(0.1 \% \mathrm{DEA}) / \mathrm{CO}_{2} 100 \mathrm{bar}, 3 \mathrm{~mL} / \mathrm{min}, \mathrm{T}_{\text {major }}=1.90 \mathrm{~min}$, $\mathrm{T}_{\text {minor }}=2.33$, ee $=96 \%$. 
(S)-2-(1-(4,4,5,5-tetramethyl-1,3,2-dioxaborolan-2-yl)octyl)-2,3-dihydro-1H-naphtho[1,8de][1,3,2]diazaborinine $\mathbf{( 2 b )}$<smiles>CCCCCCCC(B1Nc2cccc3cccc(c23)N1)B1OC(C)(C)C(C)(C)O1</smiles>

Prepared according to general procedure B. White crystalline solid. $40 \mathrm{mg}, 98 \%$ isolated yield.

${ }^{1} \mathrm{H}$ NMR $(300 \mathrm{MHz}$, Chloroform-d) $\delta 7.11$ (dd, $J=8.3,7.2 \mathrm{~Hz}, 2 \mathrm{H}), 7.01(\mathrm{dd}, J=8.3,1.1 \mathrm{~Hz}$, $2 \mathrm{H}$ ), 6.31 (dd, $J=7.3,1.1 \mathrm{~Hz}, 2 \mathrm{H}), 5.80(\mathrm{~s}, 2 \mathrm{H}), 1.57$ (dtd, $J=18.9,14.3,7.6 \mathrm{~Hz}, 2 \mathrm{H}), 1.39-$ $1.29(\mathrm{~m}, 10 \mathrm{H}), 1.27(\mathrm{~s}, 6 \mathrm{H}), 1.26(\mathrm{~s}, 6 \mathrm{H}), 0.90-0.88(\mathrm{~m}, 3 \mathrm{H}), 0.76(\mathrm{dd}, J=9.4,6.0 \mathrm{~Hz}, 1 \mathrm{H})$.

${ }^{13} \mathrm{C} \mathrm{NMR}\left(126 \mathrm{MHz}, \mathrm{CDCl}_{3}\right) \delta 141.38,136.31,127.57,119.51,117.21,105.43,83.15,32.43$, $31.90,29.71,29.26,26.43,25.05,24.89,24.51,22.71,14.18$.

HRMS (ESI+): $\mathrm{m} / z$ calculated $\mathrm{C}_{24} \mathrm{H}_{37} \mathrm{~B}_{2} \mathrm{~N}_{2} \mathrm{O}_{2}{ }^{+}\left([\mathrm{MH}]^{+}\right): 407.3035$; found: 407.3030

HPLC (Chiralcel AD-H): $40 \% \mathrm{MeOH}(0.1 \% \mathrm{DEA}) / \mathrm{CO}_{2} 100 \mathrm{bar}, 3 \mathrm{~mL} / \mathrm{min}, \mathrm{T}_{\text {major }}=1.66 \mathrm{~min}$, $\mathrm{T}_{\text {minor }}=2.08$, ee $=97 \%$. 
(S)-2-(5-((tert-butyldimethylsilyl)oxy)-1-(4,4,5,5-tetramethyl-1,3,2-dioxaborolan-2-yl)pentyl)-2,3dihydro-1 $H$-naphtho[1,8-de][1,3,2]diazaborinine (2c)<smiles></smiles>

Prepared according to general procedure B. White crystalline solid. $36 \mathrm{mg}, 75 \%$ isolated yield.

${ }^{1} \mathrm{H}$ NMR $(500 \mathrm{MHz}$, Chloroform-d) $\delta 7.09$ (dd, $J=8.2,7.3 \mathrm{~Hz}, 2 \mathrm{H}), 6.99(\mathrm{dd}, J=8.3,0.9 \mathrm{~Hz}$, $2 \mathrm{H}), 6.29$ (dd, $J=7.3,1.0 \mathrm{~Hz}, 2 \mathrm{H}), 3.64(\mathrm{td}, J=6.1,1.5 \mathrm{~Hz}, 2 \mathrm{H}), 1.70-1.47(\mathrm{~m}, 2 \mathrm{H}), 1.25(\mathrm{~s}$, $6 \mathrm{H}), 1.24(\mathrm{~s}, 6 \mathrm{H}), 0.91(\mathrm{~s}, 9 \mathrm{H}), 0.75(\mathrm{dd}, \mathrm{J}=9.0,5.2 \mathrm{~Hz}, 1 \mathrm{H}), 0.10-0.04(\mathrm{~m}, 6 \mathrm{H})$.

${ }^{13} \mathrm{C}$ NMR $\left(126 \mathrm{MHz}, \mathrm{CDCl}_{3}\right) \delta 141.45,136.39,127.66,119.61,117.32,105.55,83.31,77.16$, $63.34,35.50,29.85,26.18,25.19,24.63,22.45,18.58,-5.04$.

HRMS (ESI+): $m / z$ calculated $\mathrm{C}_{26} \mathrm{H}_{43} \mathrm{~B}_{2} \mathrm{~N}_{2} \mathrm{O}_{3} \mathrm{Si}^{+}\left([\mathrm{MH}]^{+}\right)$: 481.3224 ; found: 481.3229 .

HPLC (Chiralcel OD-H): $20 \% \mathrm{iPrOH}(0.1 \% \mathrm{DEA}) / \mathrm{CO}_{2} 100 \mathrm{bar}, 3 \mathrm{~mL} / \mathrm{min}, \mathrm{T}_{\text {major }}=3.35 \mathrm{~min}$, $\mathrm{T}_{\text {minor }}=4.30 \mathrm{~min}$, ee $=93 \%$. 
(S)-6-(1H-naphtho[1,8-de][1,3,2]diazaborinin-2(3H)-yl)-6-(4,4,5,5-tetramethyl-1,3,2dioxaborolan-2-yl)hexyl acetate (2d)<smiles>CC(=O)OCCCC[C@@H](B1Nc2cccc3cccc(c23)N1)B1OC(C)(C)C(C)(C)O1</smiles>

Prepared according to general procedure B. White crystalline solid. $34 \mathrm{mg}, 83 \%$ isolated yield.

${ }^{1} \mathrm{H}$ NMR $(500 \mathrm{MHz}$, Chloroform- $d$ ) $\delta 7.11$ (dd, $J=8.3,7.3 \mathrm{~Hz}, 2 \mathrm{H}), 7.02(\mathrm{dd}, J=8.3,1.0 \mathrm{~Hz}$, $2 \mathrm{H}), 6.32(\mathrm{dd}, J=7.3,1.0 \mathrm{~Hz}, 2 \mathrm{H}), 5.82(\mathrm{~s}, 2 \mathrm{H}), 4.10(\mathrm{dt}, J=7.9,6.7 \mathrm{~Hz}, 2 \mathrm{H}), 2.06(\mathrm{~s}, 3 \mathrm{H}), 1.75$ $-1.63(\mathrm{~m}, 4 \mathrm{H}), 1.58$ (ddt, $J=12.7,9.5,6.1 \mathrm{~Hz}, 1 \mathrm{H}), 1.43$ (dddd, $J=17.3,10.0,8.6,5.7 \mathrm{~Hz}, 1 \mathrm{H}$ ), $1.28(\mathrm{~s}, 6 \mathrm{H}), 1.26(\mathrm{~s}, 6 \mathrm{H}), 0.78(\mathrm{dd}, J=9.4,6.1 \mathrm{~Hz}, 1 \mathrm{H})$.

${ }^{13} \mathrm{C}$ NMR $\left(126 \mathrm{MHz}, \mathrm{CDCl}_{3}\right) \delta 171.31,141.27,136.28,127.57,119.50,117.29,105.49,83.25$, $64.44,28.69,28.54,26.01,25.04,24.49,21.06$.

HRMS (ESI+): $\mathrm{m} / \mathrm{z}$ calculated $\mathrm{C}_{23} \mathrm{H}_{33} \mathrm{~B}_{2} \mathrm{~N}_{2} \mathrm{O}_{4}{ }^{+}\left([\mathrm{MH}]^{+}\right): 423.2621$; found: 423.2612 .

HPLC (Chiralcel AD-H): $40 \% \mathrm{MeOH}(0.1 \% \mathrm{DEA}) / \mathrm{CO}_{2} 100 \mathrm{bar}, 3 \mathrm{~mL} / \mathrm{min}, \mathrm{T}_{\text {major }}=1.47 \mathrm{~min}$, $\mathrm{T}_{\text {minor }}=1.59 \mathrm{~min}$, ee $=97 \%$. 
2-((1S)-5-((tetrahydro-2H-pyran-2-yl)oxy)-1-(4,4,5,5-tetramethyl-1,3,2-dioxaborolan-2-yl)pentyl)2,3-dihydro-1H-naphtho[1,8-de][1,3,2]diazaborinine (2e)<smiles>CC1(C)OB([C@H](CCCO[C@H]2CCCCO2)B2Nc3cccc4cccc(c34)N2)OC1(C)C</smiles>

Prepared according to general procedure B. White crystalline solid. Isolated as a mixture of diastereomers (1:1) $39 \mathrm{mg}, 87 \%$ isolated yield.

${ }^{1} \mathrm{H}$ NMR (500 MHz, Chloroform-d) $\delta 7.09(\mathrm{t}, \mathrm{J}=7.8 \mathrm{~Hz}, 2 \mathrm{H}), 6.99(\mathrm{~d}, \mathrm{~J}=8.2 \mathrm{~Hz}, 2 \mathrm{H}), 6.29$ (d, J $=7.3 \mathrm{~Hz}, 2 \mathrm{H}), 5.86(\mathrm{~d}, J=4.0 \mathrm{~Hz}, 2 \mathrm{H}), 4.59(\mathrm{q}, J=4.0 \mathrm{~Hz}, 1 \mathrm{H}), 3.92-3.84(\mathrm{~m}, 1 \mathrm{H}), 3.77$ (ddd, $J=11.0,7.6,3.5 \mathrm{~Hz}, 1 \mathrm{H}), 3.50(\mathrm{dt}, J=10.9,4.8 \mathrm{~Hz}, 1 \mathrm{H}), 3.43(\mathrm{dt}, J=10.0,5.5 \mathrm{~Hz}, 1 \mathrm{H}), 1.92-$ $1.79(\mathrm{~m}, 1 \mathrm{H}), 1.77-1.46(\mathrm{~m}, 9 \mathrm{H}), 1.25(\mathrm{~s}, 6 \mathrm{H}), 1.24(\mathrm{~s}, 6 \mathrm{H}), 0.82-0.74(\mathrm{~m}, 1 \mathrm{H})$.

${ }^{13} \mathrm{C}$ NMR (126 MHz, $\mathrm{CDCl}_{3}$ Mixture of diastereomers (1:1)) $\delta[141.45,141.44], 136.38,127.64$, 119.60, [117.31, 117.30], 105.53, 99.08, 83.31, [67.67, 67.65], [62.61, 62.58], [32.28, 32.24], $30.94,25.61$, [25.16, 25.15], 24.63, [22.95, 22.88], [19.93, 19.89].

HRMS (ESI+): $\mathrm{m} / z$ calculated $\mathrm{C}_{25} \mathrm{H}_{37} \mathrm{~B}_{2} \mathrm{~N}_{2} \mathrm{O}_{4}{ }^{+}\left([\mathrm{MH}]^{+}\right): 451.2934$; found: 451.2931

HPLC (Chiralcel OD-H): $25 \% \mathrm{MeOH}(0.1 \% \mathrm{DEA}) / \mathrm{CO}_{2} 100 \mathrm{bar}, 3 \mathrm{~mL} / \mathrm{min}, \mathrm{T}_{\text {major }}=6.05,6.36$ $\min , \mathrm{T}_{\text {minor }}=6.75,7.76 \mathrm{~min}$, ee $=91 \%$. 
(S)-2-(6-(1H-naphtho[1,8-de][1,3,2]diazaborinin-2(3H)-yl)-6-(4,4,5,5-tetramethyl-1,3,2dioxaborolan-2-yl)hexyl)isoindoline-1,3-dione (2f)<smiles>CC1(C)OB([C@H](CCCCN2C(=O)c3ccccc3C2=O)B2Nc3cccc4cccc(c34)N2)OC1(C)C</smiles>

Prepared according to general procedure B. Red-orange solid. $46 \mathrm{mg}, 90 \%$ isolated yield.

${ }^{1} \mathrm{H}$ NMR $(500 \mathrm{MHz}$, Chloroform- $d$ ) $\delta 7.11$ (dd, $J=8.3,7.3 \mathrm{~Hz}, 2 \mathrm{H}), 7.02(\mathrm{dd}, J=8.3,1.0 \mathrm{~Hz}$, $2 \mathrm{H}), 6.32(\mathrm{dd}, J=7.3,1.0 \mathrm{~Hz}, 2 \mathrm{H}), 5.82(\mathrm{~s}, 2 \mathrm{H}), 4.10(\mathrm{dt}, J=7.9,6.7 \mathrm{~Hz}, 2 \mathrm{H}), 2.06(\mathrm{~s}, 3 \mathrm{H}), 1.75$ $-1.63(\mathrm{~m}, 4 \mathrm{H}), 1.58$ (ddt, $J=12.7,9.5,6.1 \mathrm{~Hz}, 1 \mathrm{H}), 1.43$ (dddd, $J=17.3,10.0,8.6,5.7 \mathrm{~Hz}, 1 \mathrm{H}$ ), $1.28(\mathrm{~s}, 6 \mathrm{H}), 1.26(\mathrm{~s}, 6 \mathrm{H}), 0.78(\mathrm{dd}, J=9.4,6.1 \mathrm{~Hz}, 1 \mathrm{H})$.

${ }^{13} \mathrm{C}$ NMR $\left(126 \mathrm{MHz}, \mathrm{CDCl}_{3}\right) \delta 171.31,141.27,136.28,127.57,119.50,117.29,105.49,83.25$, $64.44,28.69,28.54,26.01,25.04,24.49,21.06$.

HRMS (ESI+): $m / z$ calculated $\mathrm{C}_{29} \mathrm{H}_{33} \mathrm{~B}_{2} \mathrm{~N}_{3} \mathrm{O}_{4}{ }^{+}\left([\mathrm{M}]^{+}\right)$: 509.2658 ; found: 509.2652

HPLC (Chiralcel OJ-H): $40 \% \mathrm{MeOH}(0.1 \% \mathrm{DEA}) / \mathrm{CO}_{2} 100 \mathrm{bar}, 3 \mathrm{~mL} / \mathrm{min}, \mathrm{T}_{\text {major }}=5.33 \mathrm{~min}$, $\mathrm{T}_{\text {minor }}=6.52$, ee $=97 \%$. 
2-((1S,4R)-4,8-dimethyl-1-(4,4,5,5-tetramethyl-1,3,2-dioxaborolan-2-yl)non-7-en-1-yl)-2,3dihydro-1 $H$-naphtho[1,8-de][1,3,2]diazaborinine $(\mathbf{2 g})$<smiles>CC(C)=CCC[C@H](C)CC[C@H](B1Nc2cccc3cccc(c23)N1)B1OC(C)(C)C(C)(C)O1</smiles>

Prepared according to general procedure B. White crystalline solid. $45 \mathrm{mg}, 98 \%$.

${ }^{1} \mathrm{H}$ NMR $(500 \mathrm{MHz}$, Chloroform-d) $\delta 7.09(\mathrm{t}, J=7.8 \mathrm{~Hz}, 2 \mathrm{H}), 6.99(\mathrm{~d}, J=8.2 \mathrm{~Hz}, 2 \mathrm{H}), 6.29(\mathrm{~d}, J$ $=7.3 \mathrm{~Hz}, 2 \mathrm{H}), 5.78(\mathrm{~s}, 2 \mathrm{H}), 5.11(\mathrm{t}, J=7.3 \mathrm{~Hz}, 1 \mathrm{H}), 1.97(\mathrm{dt}, J=24.7,7.5 \mathrm{~Hz}, 2 \mathrm{H}), 1.69(\mathrm{~s}, 3 \mathrm{H})$, $1.60(\mathrm{~s}, 3 \mathrm{H}), 1.59-1.53(\mathrm{~m}, 3 \mathrm{H}), 1.43(\mathrm{dt}, J=12.7,6.3 \mathrm{~Hz}, 1 \mathrm{H}), 1.39-1.31(\mathrm{~m}, 1 \mathrm{H}), 1.27(\mathrm{~s}$, $1 \mathrm{H}), 1.25(\mathrm{~s}, 6 \mathrm{H}), 1.24(\mathrm{~s}, 6 \mathrm{H}), 0.89(\mathrm{~d}, J=6.3 \mathrm{~Hz}, 4 \mathrm{H}), 0.69(\mathrm{t}, J=7.7 \mathrm{~Hz}, 1 \mathrm{H})$.

${ }^{13} \mathrm{C}$ NMR $\left(126 \mathrm{MHz}, \mathrm{CDCl}_{3}\right) \delta 141.37,136.29,131.06,127.56,125.04,119.50,117.21,105.43$, 83.15, 39.88, 37.00, 32.66, 25.76, 25.52, 25.06, 24.49, 23.91, 19.79, 17.69.

HRMS (ESI+): $m / z$ calculated $\mathrm{C}_{27} \mathrm{H}_{41} \mathrm{~B}_{2} \mathrm{~N}_{2} \mathrm{O}_{2}{ }^{+}\left([\mathrm{MH}]^{+}\right): 447.3349$; found: 447.3343 
(S)-2-(4-phenyl-1-(4,4,5,5-tetramethyl-1,3,2-dioxaborolan-2-yl)butyl)-2,3-dihydro-1Hnaphtho[1,8-de][1,3,2]diazaborinine $(\mathbf{2 h})$<smiles>CC1(C)OB([C@H](CCCc2ccccc2)B2Nc3cccc4cccc(c34)N2)OC1(C)C</smiles>

Prepared according to general procedure B. White crystalline solid. $36 \mathrm{mg}, 84 \%$ isolated yield.

${ }^{1} \mathrm{H}$ NMR $(500 \mathrm{MHz}$, Chloroform-d) $\delta 7.32(\mathrm{dd}, J=8.2,6.9 \mathrm{~Hz}, 2 \mathrm{H}), 7.26-7.19(\mathrm{~m}, 3 \mathrm{H}), 7.12$ (dd, $J=8.3,7.3 \mathrm{~Hz}, 2 \mathrm{H}), 7.03$ (dd, $J=8.2,1.0 \mathrm{~Hz}, 2 \mathrm{H}), 6.30$ (dd, $J=7.3,1.0 \mathrm{~Hz}, 2 \mathrm{H}), 5.79(\mathrm{~s}$, $2 \mathrm{H}), 2.68(\mathrm{t}, J=6.9 \mathrm{~Hz}, 2 \mathrm{H}), 1.82-1.66(\mathrm{~m}, 3 \mathrm{H}), 1.64-1.54(\mathrm{~m}, 1 \mathrm{H}), 1.29(\mathrm{~s}, 6 \mathrm{H}), 1.27(\mathrm{~s}, 6 \mathrm{H})$, $0.82(\mathrm{dd}, J=8.2,6.0 \mathrm{~Hz}, 1 \mathrm{H})$.

${ }^{13} \mathrm{C} \mathrm{NMR}\left(126 \mathrm{MHz}, \mathrm{CDCl}_{3}\right) \delta 142.63,141.31,136.29,128.45,128.35,127.57,125.69,119.51$, $117.26,105.48,83.24,36.00,34.03,25.94,25.08,24.53$.

HRMS (ESI+): $\mathrm{m} / \mathrm{z}$ calculated $\mathrm{C}_{26} \mathrm{H}_{33} \mathrm{~B}_{2} \mathrm{~N}_{2} \mathrm{O}_{2}{ }^{+}\left([\mathrm{MH}]^{+}\right): 427.2723$; found: 427.2722 .

HPLC (Chiralcel OJ-H): $40 \% \mathrm{MeOH}(0.1 \% \mathrm{DEA}) / \mathrm{CO}_{2} 100 \mathrm{bar}, 3 \mathrm{~mL} / \mathrm{min}, \mathrm{T}_{\text {major }}=3.60 \mathrm{~min}$, $\mathrm{T}_{\text {minor }}=5.65$, ee $=97 \%$. 
(S)-2-(3-phenyl-1-(4,4,5,5-tetramethyl-1,3,2-dioxaborolan-2-yl)propyl)-2,3-dihydro-1Hnaphtho[1,8-de][1,3,2]diazaborinine (2i)<smiles></smiles>

Prepared according to general procedure B. White crystalline solid. $93 \%, 38 \mathrm{mg}$.

${ }^{1} \mathrm{H}$ NMR $(500 \mathrm{MHz}$, Chloroform- $d$ ) $\delta 7.36-7.30(\mathrm{~m}, 2 \mathrm{H}), 7.27-7.21(\mathrm{~m}, 3 \mathrm{H}), 7.12(\mathrm{dd}, J=8.3$, $7.3 \mathrm{~Hz}, 2 \mathrm{H}$ ), 7.03 (dd, $J=8.3,1.0 \mathrm{~Hz}, 2 \mathrm{H}$ ), 6.31 (dd, $J=7.4,1.0 \mathrm{~Hz}, 2 \mathrm{H}$ ), 5.81 (s, 2H), 2.76 (dtd, $J=16.3,10.7,10.2,6.5 \mathrm{~Hz}, 1 \mathrm{H}$ ), 2.64 (ddd, $J=13.4,9.8,6.4 \mathrm{~Hz}, 1 \mathrm{H}$ ), 2.01 (dtd, $J=13.1,9.8$, $5.6 \mathrm{~Hz}, 1 \mathrm{H}), 1.92-1.73(\mathrm{~m}, 1 \mathrm{H}), 1.31(\mathrm{~s}, 6 \mathrm{H}), 1.30(\mathrm{~s}, 6 \mathrm{H}), 0.86(\mathrm{dd}, J=9.8,5.7 \mathrm{~Hz}, 1 \mathrm{H}) .{ }^{13} \mathrm{C}$ $\operatorname{NMR}\left(126 \mathrm{MHz}, \mathrm{CDCl}_{3}\right)$

${ }^{13} \mathrm{C} \mathrm{NMR}\left(126 \mathrm{MHz}, \mathrm{CDCl}_{3}\right) \delta 142.57,141.28,136.29,128.56,128.36,127.58,125.84,119.52$, $117.31,105.50,83.30,38.47,28.58,25.16,24.54$.

HRMS (ESI+): $\mathrm{m} / \mathrm{z}$ calculated $\mathrm{C}_{25} \mathrm{H}_{31} \mathrm{~B}_{2} \mathrm{~N}_{2} \mathrm{O}_{2}{ }^{+}\left([\mathrm{MH}]^{+}\right): 413.2566$; found: 413.2562 .

HPLC (Chiralcel DD-H): $30 \% \mathrm{EtOH}(0.1 \% \mathrm{DEA}) / \mathrm{CO}_{2} 100 \mathrm{bar}, 3 \mathrm{~mL} / \mathrm{min}, \mathrm{T}_{\text {major }}=3.24 \mathrm{~min}$, $\mathrm{T}_{\text {minor }}=3.53$, ee $=98 \%$. 
(S)-2-(2-phenyl-1-(4,4,5,5-tetramethyl-1,3,2-dioxaborolan-2-yl)ethyl)-2,3-dihydro-1 $\mathrm{H}$ naphtho[1,8-de][1,3,2]diazaborinine $(\mathbf{2 j})$<smiles>CC1(C)OB([C@H](Cc2ccccc2)B2Nc3cccc4cccc(c34)N2)OC1(C)C</smiles>

Prepared according to a slightly modified general procedure B. Reaction conducted at 0.025 mmol scale using $10 \mathrm{~mol} \%{ }^{\mathrm{tBu}} \mathrm{CoCM}$ in J-Young Tube under constant vertical rotation. White crystalline solid. $99 \%, 9 \mathrm{mg}$.

${ }^{1} \mathrm{H}$ NMR $(500 \mathrm{MHz}$, Chloroform- $d) \delta 7.25(\mathrm{~s}, 4 \mathrm{H}), 7.16(\mathrm{q}, J=4.4 \mathrm{~Hz}, 1 \mathrm{H}), 7.08(\mathrm{t}, J=7.8 \mathrm{~Hz}$, $2 \mathrm{H}), 7.02-6.94(\mathrm{~m}, 2 \mathrm{H}), 6.26(\mathrm{dd}, J=7.3,1.1 \mathrm{~Hz}, 2 \mathrm{H}), 5.80(\mathrm{~s}, 2 \mathrm{H}), 2.95(\mathrm{dd}, J=14.1,9.5 \mathrm{~Hz}$, $1 \mathrm{H}), 2.88(\mathrm{dd}, J=14.1,6.8 \mathrm{~Hz}, 1 \mathrm{H}), 1.18(\mathrm{~s}, 6 \mathrm{H}), 1.16(\mathrm{~s}, 6 \mathrm{H}), 0.84(\mathrm{t}, J=6.3 \mathrm{~Hz}, 1 \mathrm{H})$.

${ }^{13} \mathrm{C}$ NMR $\left(126 \mathrm{MHz}, \mathrm{CDCl}_{3}\right) \delta 142.67,140.15,135.22,127.23,127.16,126.51,124.71,118.47$, $116.29,104.48,82.33,76.24,75.99,75.73,28.68,23.90,23.45$.

HRMS (ESI+): $\mathrm{m} / \mathrm{z}$ calculated $\mathrm{C}_{24} \mathrm{H}_{29} \mathrm{~B}_{2} \mathrm{~N}_{2} \mathrm{O}_{2}{ }^{+}\left([\mathrm{MH}]^{+}\right): 399.2410$; found: 399.2391 .

HPLC (Chiralcel AD-H): $40 \% \mathrm{MeOH}(0.1 \% \mathrm{DEA}) / \mathrm{CO}_{2} 100 \mathrm{bar}, 3 \mathrm{~mL} / \mathrm{min}, \mathrm{T}_{\text {major }}=2.34 \mathrm{~min}$, $\mathrm{T}_{\text {minor }}=4.29$, ee $=98 \%$. 
(S)-2-(2-cyclopentyl-1-(4,4,5,5-tetramethyl-1,3,2-dioxaborolan-2-yl)ethyl)-2,3-dihydro-1Hnaphtho[1,8-de][1,3,2]diazaborinine (2k)<smiles>CC1(C)OB([C@H](CC2CCCC2)B2Nc3cccc4cccc(c34)N2)OC1(C)C</smiles>

Prepared according to general procedure B using $10 \mathrm{~mol} \%{ }^{\mathrm{tB}}{ }^{\mathrm{C}} \mathrm{CoCM}$. White Solid, $90 \%, 35 \mathrm{mg}$. Single clear crystals suitable for $\mathrm{x}$-ray crystallography were grown by diffusion of pentane into a solution of ethyl acetate.

${ }^{1} \mathrm{H}$ NMR $(500 \mathrm{MHz}$, Chloroform- $d$ ) $\delta 7.01$ (dd, $J=8.3,7.3 \mathrm{~Hz}, 2 \mathrm{H}), 6.91(\mathrm{dd}, J=8.3,1.0 \mathrm{~Hz}$, 2H), 6.21 (dd, $J=7.3,1.0 \mathrm{~Hz}, 2 \mathrm{H}), 5.69(\mathrm{~s}, 2 \mathrm{H}), 1.77-1.64(\mathrm{~m}, 4 \mathrm{H}), 1.61$ (ddd, $J=13.2,9.3$, $6.4 \mathrm{~Hz}, 1 \mathrm{H}), 1.56-1.47(\mathrm{~m}, 2 \mathrm{H}), 1.47-1.33(\mathrm{~m}, 2 \mathrm{H}), 1.17(\mathrm{~s}, 6 \mathrm{H}), 1.16(\mathrm{~s}, 6 \mathrm{H}), 1.09-0.93(\mathrm{~m}$, $2 \mathrm{H}), 0.75(\mathrm{dd}, J=9.3,6.3 \mathrm{~Hz}, 1 \mathrm{H})$.

${ }^{13} \mathrm{C}$ NMR $\left(126 \mathrm{MHz}, \mathrm{CDCl}_{3}\right) \delta 141.37,136.30,127.57,119.50,117.22,105.44,83.17,42.41$, $32.84,32.64,32.54,25.23,25.20,24.98,24.58$.

HRMS (ESI+): $\mathrm{m} / \mathrm{z}$ calculated $\mathrm{C}_{23} \mathrm{H}_{33} \mathrm{~B}_{2} \mathrm{~N}_{2} \mathrm{O}_{2}{ }^{+}\left([\mathrm{MH}]^{+}\right): 391.2723$; found: 391.2732 .

HPLC (Chiralcel AD-H): $20 \% \mathrm{MeOH}(0.1 \% \mathrm{DEA}) / \mathrm{CO}_{2} 100 \mathrm{bar}, 3 \mathrm{~mL} / \mathrm{min}, \mathrm{T}_{\text {major }}=2.60 \mathrm{~min}$, $\mathrm{T}_{\text {minor }}=3.20$, ee $=94 \%$.

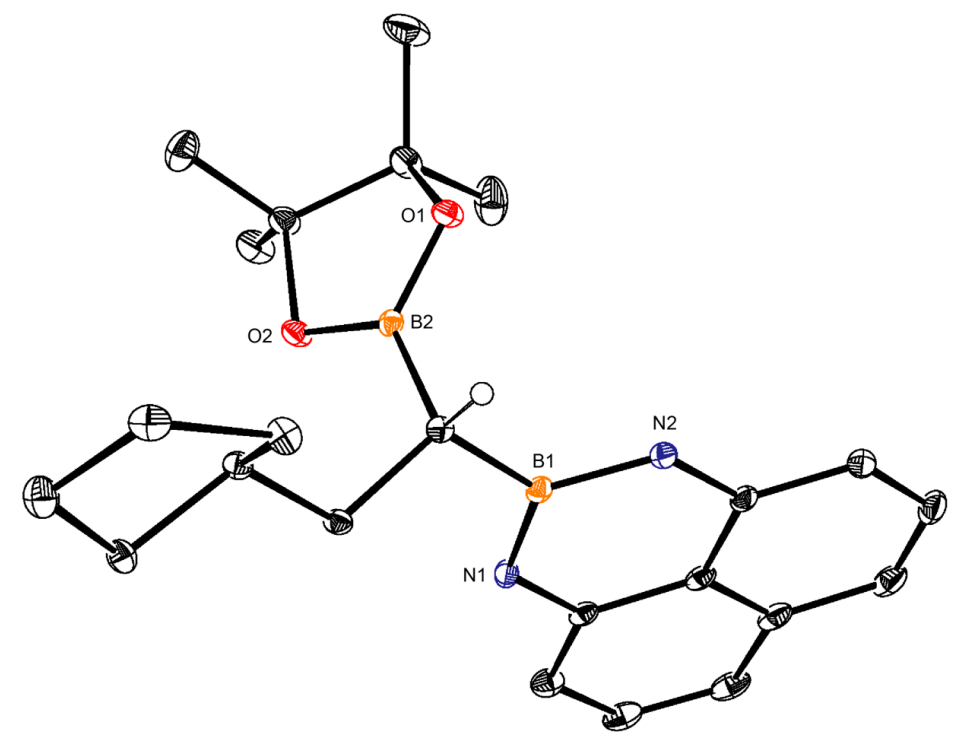

Figure S1. Solid state structure of $\mathbf{2 k}$. Thermal Ellipsoids at $30 \%$ probability. Non-stereogenic $\mathrm{H}$-atoms omitted for clarity. 
(S)-2-(2-cyclohexyl-1-(4,4,5,5-tetramethyl-1,3,2-dioxaborolan-2-yl)ethyl)-2,3-dihydro-1Hnaphtho[1,8-de][1,3,2]diazaborinine (2I)<smiles>CC1(C)OB([C@H](CC2CCCCC2)B2Nc3cccc4cccc(c34)N2)OC1(C)C</smiles>

Prepared according to general procedure B. Using $10 \mathrm{~mol} \%{ }^{\mathrm{tBu}} \mathrm{CoCM}$. White crystalline solid. 37 $\mathrm{mg}, 98 \%$ isolated yield.

${ }^{1} \mathrm{H}$ NMR $(300 \mathrm{MHz}$, Chloroform- $d) \delta 7.11(\mathrm{t}, J=7.7 \mathrm{~Hz}, 2 \mathrm{H}), 7.05-6.90(\mathrm{~m}, 2 \mathrm{H}), 6.35-6.15$ $(\mathrm{m}, 2 \mathrm{H}), 5.78(\mathrm{~s}, 2 \mathrm{H}), 1.92-1.56(\mathrm{~m}, 7 \mathrm{H}), 1.26(\mathrm{~s}, 6 \mathrm{H}), 1.25(\mathrm{~s}, 6 \mathrm{H}), 1.18(\mathrm{~d}, J=6.7 \mathrm{~Hz}, 3 \mathrm{H})$, $0.97-0.79(\mathrm{~m}, 4 \mathrm{H})$.

${ }^{13} \mathrm{C}$ NMR $\left(126 \mathrm{MHz}, \mathrm{CDCl}_{3}\right) \delta 141.37,136.27,127.56,119.47,117.19,105.42,83.16,39.63$, $33.85,33.58,33.08,26.70,26.42,24.98,24.50$.

HRMS (ESI+): $\mathrm{m} / \mathrm{z}$ calculated $\mathrm{C}_{24} \mathrm{H}_{35} \mathrm{~B}_{2} \mathrm{~N}_{2} \mathrm{O}_{2}{ }^{+}\left([\mathrm{MH}]^{+}\right): 405.2879$; found: 405.2887 .

HPLC (Chiralcel OJ-H): $40 \%$ EtOH (0.1 \% DEA)/CO $100 \mathrm{bar}, 3 \mathrm{~mL} / \mathrm{min}, \mathrm{T}_{\text {major }}=1.71 \mathrm{~min}$, $\mathrm{T}_{\text {minor }}=2.30$, ee $=98 \%$. 
(S)-2,2'-(1-(4,4,5,5-tetramethyl-1,3,2-dioxaborolan-2-yl)ethane-1,2-diyl)bis(2,3-dihydro-1H-naphtho[1,8de][1,3,2]diazaborinine) $(2 \mathrm{~m})$<smiles>CC1(C)OB(C(CB2Nc3cccc4cccc(c34)N2)B2Nc3cccc4cccc(c34)N2)OC1(C)C</smiles>

Prepared according to general procedure B using $10 \mathrm{~mol} \%{ }^{\mathrm{tBu}} \mathrm{CoCM}$. White crystalline solid. 24 $\mathrm{mg}, 50 \%$ isolated yield. Single purple crystals suitable for x-ray crystallography were grown by diffusion of pentane into a solution of ethyl acetate.

${ }^{1} \mathrm{H}$ NMR (500 MHz, Chloroform-d) $\delta 7.13(\mathrm{t}, J=7.8 \mathrm{~Hz}, 4 \mathrm{H}), 7.03(\mathrm{~d}, \mathrm{~J}=8.2 \mathrm{~Hz}, 4 \mathrm{H}), 6.33(\mathrm{~d}, J$ $=7.5 \mathrm{~Hz}, 2 \mathrm{H}), 6.31(\mathrm{~d}, J=7.5 \mathrm{~Hz}, 2 \mathrm{H}), 5.94(\mathrm{~s}, 2 \mathrm{H}), 5.90(\mathrm{~s}, 2 \mathrm{H}), 1.30(\mathrm{~s}, 12 \mathrm{H}), 1.13(\mathrm{dd}, J=$ $14.9,11.2 \mathrm{~Hz}, 1 \mathrm{H}), 1.03$ (dd, $J=15.0,4.0 \mathrm{~Hz}, 1 \mathrm{H}), 0.89$ (dd, $J=11.4,4.2 \mathrm{~Hz}, 1 \mathrm{H})$.

${ }^{13} \mathrm{C}$ NMR $\left(126 \mathrm{MHz}, \mathrm{CDCl}_{3}\right) \delta 141.30,141.26,136.37,136.31,127.61,119.63,119.50,117.38$, 117.34, 105.57, 105.47, 83.64, 29.74.

${ }^{11}$ B NMR (96 MHz, $\left.\mathrm{CDCl}_{3}\right) \delta 29.44,-9.28$.

HRMS (ESI+): $m / z$ calculated for $\mathrm{C}_{28} \mathrm{H}_{31} \mathrm{~B}_{3} \mathrm{~N}_{4} \mathrm{O}_{2}{ }^{+}\left([\mathrm{M}]^{+}\right): 488.2721$; found: 488.2728 .

HPLC (Chiralcel IC): $40 \% \mathrm{MeOH}(0.1 \% \mathrm{DEA}) / \mathrm{CO}_{2} 100 \mathrm{bar}, 3 \mathrm{~mL} / \mathrm{min}, \mathrm{T}_{\text {major }}=8.05 \mathrm{~min}, \mathrm{~T}_{\text {minor }}$ $=7.10$, ee $=85 \%$. 


\section{Functionalization of 1,1-diboryl alkane}

Preparation of trifluoroborate salt: To a $20 \mathrm{~mL}$ scintillation vial containing a Teflon-coated magnetic stir bar was added 1a (500 mg, 1.27 mmol, 1 equiv.), and acetonitrile (10.2 mL, 0.125 M). After 1a was completely dissolved, saturated aqueous $\mathrm{KHF}_{2}(4.5 \mathrm{M}, 12.75 \mathrm{mmol}, 10$ equiv.) was added dropwise, and the reaction vigorously stirred for $2 \mathrm{~h}$. The solvent was then removed in vacuo, and the contents of the vial dissolved again in acetonitrile, and an equivalent amount of water added. The solvent was removed again, and this process was repeated 5 times. Complete removal of pinacol was required for subsequent reactivity. The solid residue was dissolved in acetone, and filtered through a cotton plug, and the solvent removed affording an off-white solid ( $293 \mathrm{mg}, 62 \%$ crude yield) that was used directly in the next step.

Suzuki-Miyaura Cross Coupling: In two separate septum capped $4 \mathrm{~mL}$ vials containing Teflon-coated magnetic stir bars, was added trifluoroborate salt $(146 \mathrm{mg}, 0.39 \mathrm{mmol}$ crude, 1.5 equiv.), $\mathrm{K}_{2} \mathrm{CO}_{3}$ (109 mg, $0.787 \mathrm{mmol}, 3$ equiv.), Pd-G3 (9.7 mg, $0.0125 \mathrm{mmol}, 0.05$ equiv.), and XPhos (25 mg, $0.05 \mathrm{mmol}, 0.2$ equiv.) or PAd 3 (23 mg, $0.05 \mathrm{mmol}, 0.2$ equiv.). The vial was placed under a flow of argon, degassed toluene $(0.75 \mathrm{~mL})$ was added along with phenyl iodide (0.029 mL, $0.262 \mathrm{mmol}, 1$ equiv.). Degassed $\mathrm{H}_{2} \mathrm{O}(0.25 \mathrm{~mL})$ was added, and the reaction mixture sparged with argon for 5 minutes. The vials were then sealed with parafilm wax and electric tape, and stirred at $80^{\circ} \mathrm{C}$ for $17 \mathrm{~h}$. Both reactions were quenched by passing their contents through a silica gel pad, eluting with DCM. The product was purified by silica-gel column chromatography (ethyl acetate:hexanes 1:50). 
(S)-2-(1-phenylheptyl)-2,3-dihydro-1 H-naphtho[1,8-de][1,3,2]diazaborinine ((R)-Ph-2a):

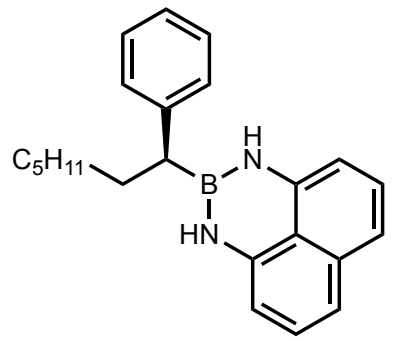

With $\mathrm{PAd}_{3} .93 \%$ isolated yield, $84 \%$ ee.

With XPhos. $49 \%$ isolated yield, $32 \%$ ee. The spectra matched that above.

${ }^{1} \mathrm{H}$ NMR (500 MHz, Chloroform-d) $\delta .7 .31(\mathrm{t}, J=7.6 \mathrm{~Hz}, 2 \mathrm{H}), 7.21-7.14(\mathrm{~m}, 3 \mathrm{H}), 7.07$ (t, J = 7.8 Hz, 2H), 6.99 (d, J = 8.2 Hz, 2H), 6.25 (d, J = 7.3 Hz, 2H), 5.50 (s, 2H), 2.36 $(\mathrm{t}, J=7.9 \mathrm{~Hz}, 1 \mathrm{H}), 1.86-1.71(\mathrm{~m}, 2 \mathrm{H}), 1.34-1.14(\mathrm{~m}, 13 \mathrm{H}), 0.86(\mathrm{t}, J=6.9 \mathrm{~Hz}, 3 \mathrm{H})$.

${ }^{13} \mathrm{C}$ NMR (126 MHz, $\left.\mathrm{CDCl}_{3}\right)$ ठ. 144.00, 141.00, 136.25, 129.80, 128.29, 127.57, 125.41, $119.49,117.55,105.72,31.75,31.62,29.39,29.15,22.69,14.11$.

HRMS (ESI+): $m / 2$ calculated for $\mathrm{C}_{23} \mathrm{H}_{27} \mathrm{~B}_{1} \mathrm{~N}_{2}{ }^{+}\left([\mathrm{M}]^{+}\right)$: 342.2267 ; found: 342.2276 .

HPLC (Chiralcel OD-H): $40 \% \mathrm{EtOH}(0.1 \% \mathrm{DEA}) / \mathrm{CO}_{2} 100 \mathrm{bar}, 3 \mathrm{~mL} / \mathrm{min}, \mathrm{T}_{\text {major }}=3.82 \mathrm{~min}$, $\mathrm{T}_{\text {minor }}=3.40$.

Deprotection and oxidation to determine absolute stereochemistry was preformed using literature procuedure. ${ }^{5}$ 

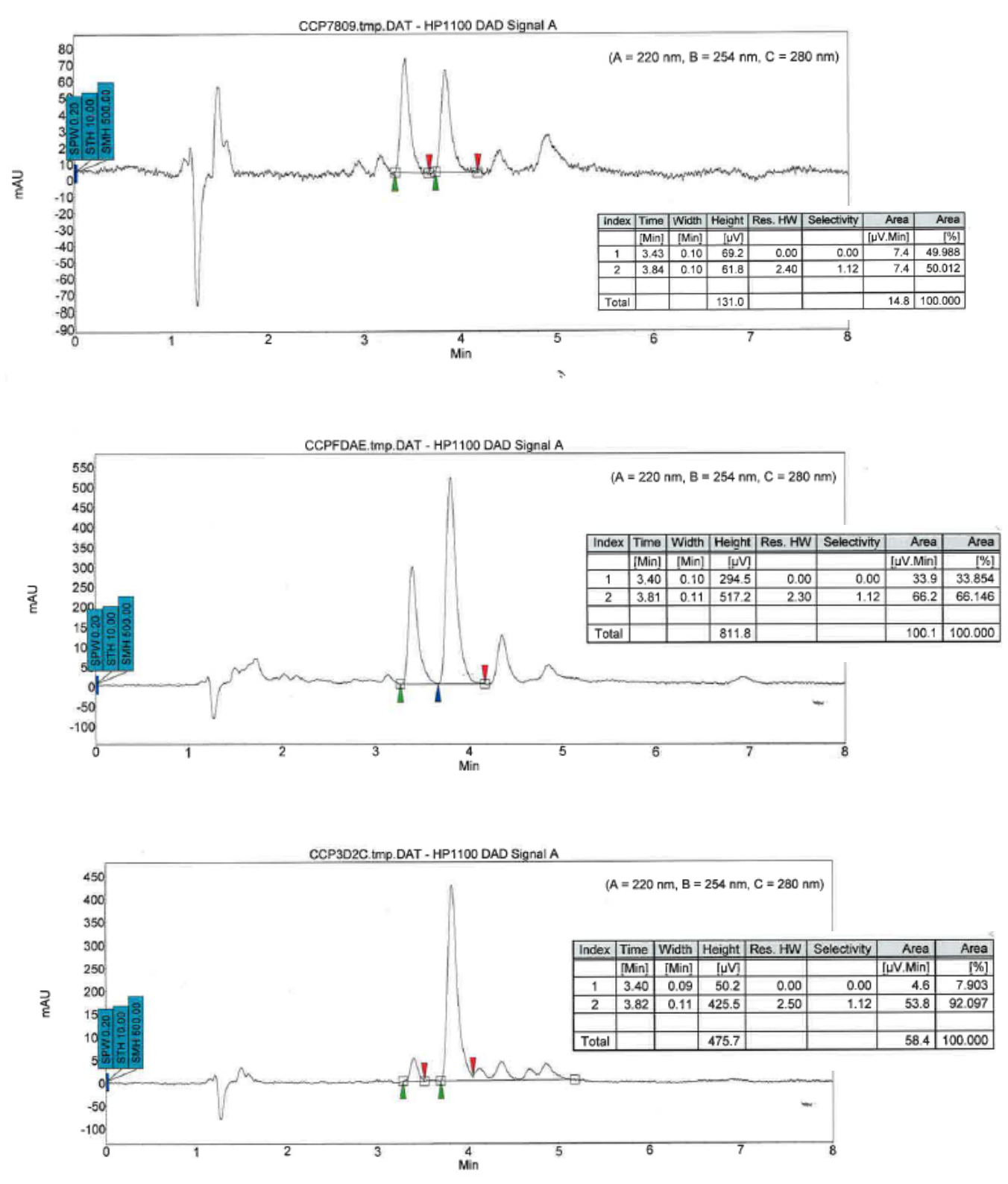

Figure S2. Chiral Chromatogram of rac-Ph-2a (top), (S)-Ph-2a from XPhos, (S)-Ph-2a from

$\mathrm{PAd}_{3}$ 

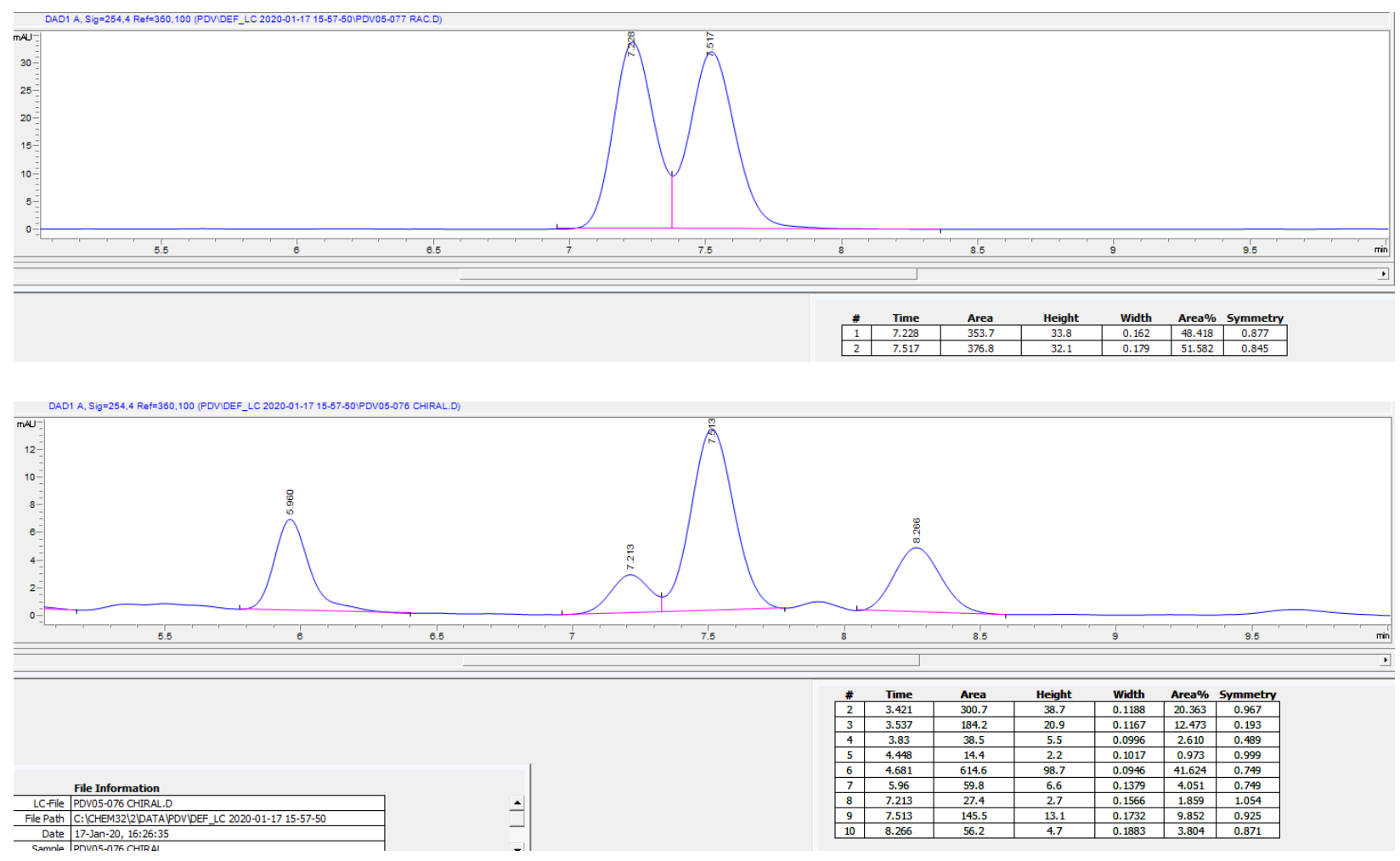

Figure S3. Chiral Chromatogram of rac-1-phenylheptan-1-ol (top), (S)-1-phenylheptan-1-ol from deprotection and oxidation (bottom). $5 \%$ IPA, $\mathrm{T}_{\text {major }}=7.51 \mathrm{~min}, \mathrm{~T}_{\operatorname{minor}}=7.21 \mathrm{~min}(68 \%$ ee). ${ }^{9}$ 


\section{Hydrogenation of Alkenyl Boronates}

\section{Hydrogenation of Vinyl Boronates}

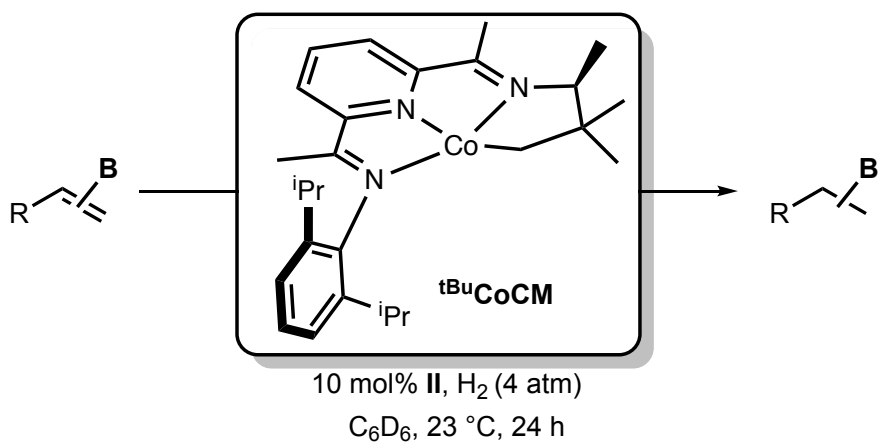

General Procedure C - To a $20 \mathrm{~mL}$ vial containing cobalt complex ${ }^{\mathrm{tBu}} \mathrm{CoCM}$ was added 1,1diborylalkene substrate $1(0.025 \mathrm{mmol})$ and $\mathrm{C}_{6} \mathrm{D}_{6}(0.3 \mathrm{~mL})$. The solution was transferred to a JYoung tube with a $1 \mathrm{~mL}$ syringe, and the vial was washed with benzene $(1 \times 0.1 \mathrm{~mL})$, so that the total volume of the reaction solution was $0.4 \mathrm{~mL}$. The J-Young tube was sealed and brought out of the glovebox and connected to a high vacuum line. The J-Young tube was frozen in liquid nitrogen, evacuated, and backfilled with 1 atm of hydrogen gas at liquid nitrogen temperature, corresponding to approximately 4 atm of hydrogen pressure at $23^{\circ} \mathrm{C}$. The frozen reaction solution was then left to thaw, after which it was vertically rotated for $24 \mathrm{~h}$. The color of the reaction solution under an atmosphere of hydrogen was purple (the same shade of purple as when cobalt complex ${ }^{\mathrm{tBu}} \mathrm{CoCM}$ is dissolved in benzene). After this time, conversion was measured directly by ${ }^{1} \mathrm{H}$ NMR by relative integration of starting material and product. If significant broadening complicates analysis, the mixture was filtered through silica and eluted with DCM. The mixture was then analyzed by ${ }^{1} \mathrm{H}$ NMR. In all cases ${ }^{\mathrm{tBu}} \mathrm{CoCM}$ is still observed in solution after the allocated reaction time. 

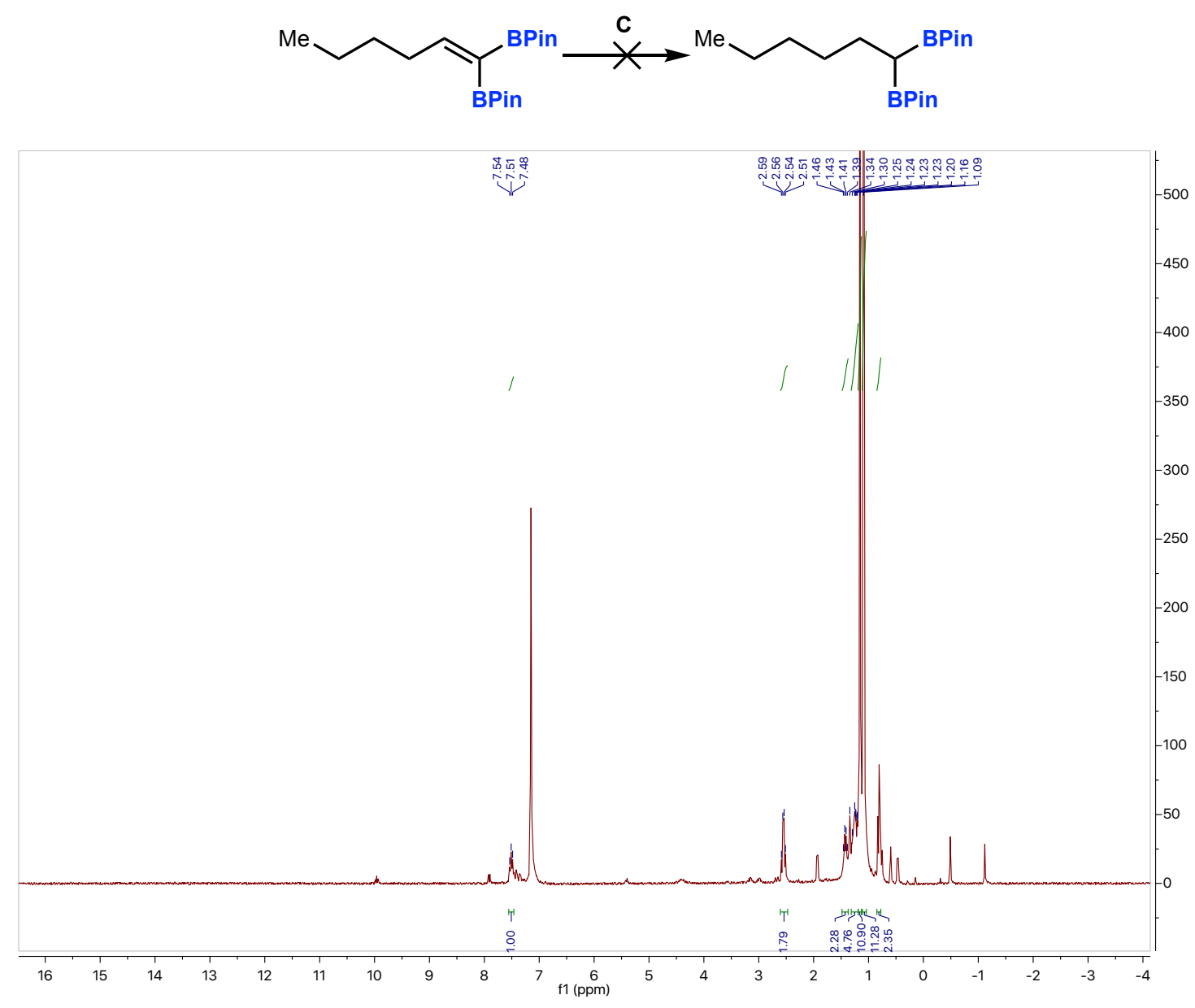

Figure S4. 1H NMR Spectrum of failed hydrogenation of 3a. Catalyst observed at $\delta=-0.49$, 1.12 indicates no catalyst death. 


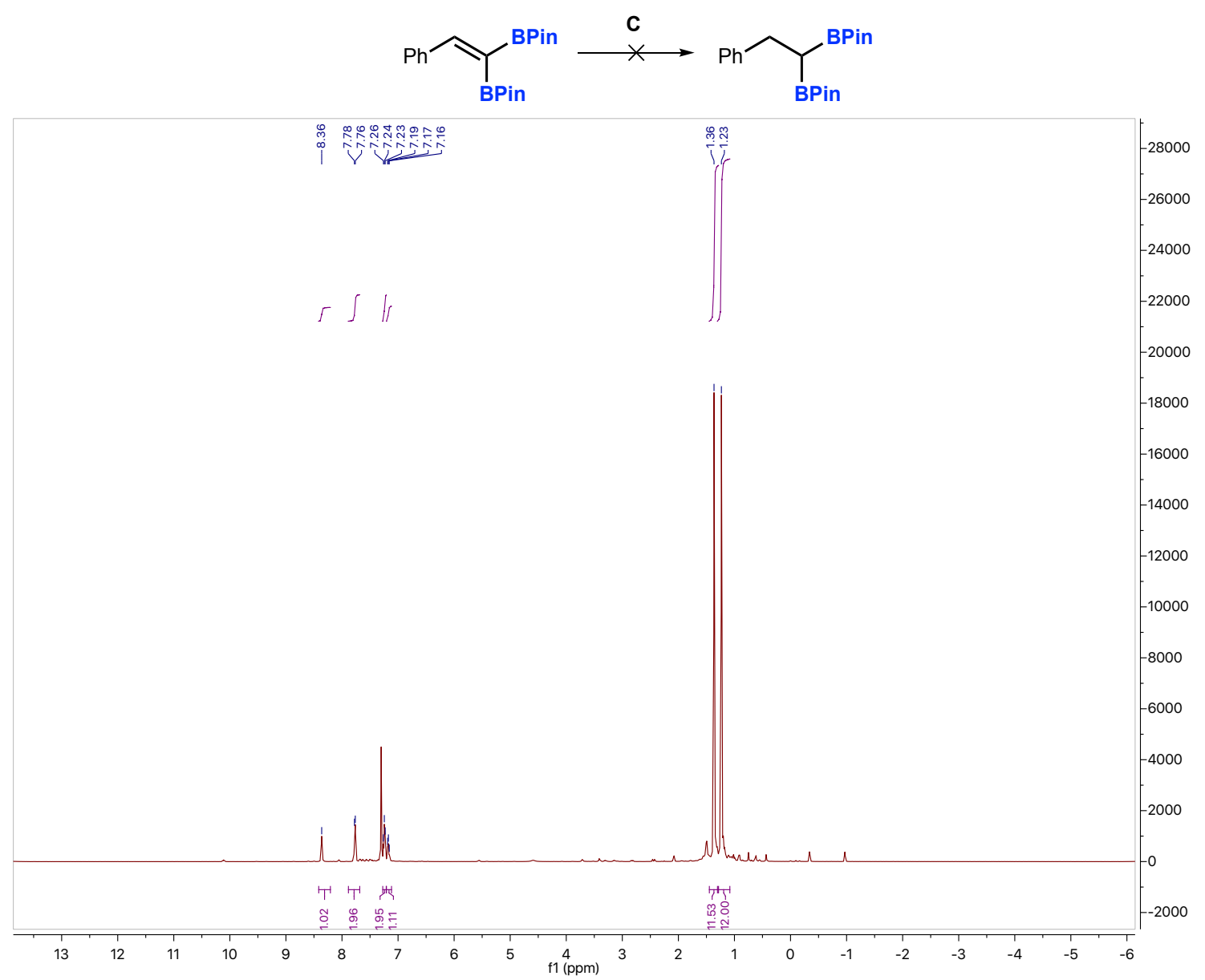

Figure S5. 1H NMR Spectrum of failed hydrogenation of 3b. Catalyst observed at $\delta=-0.49$, 1.12 indicates no catalyst death. 


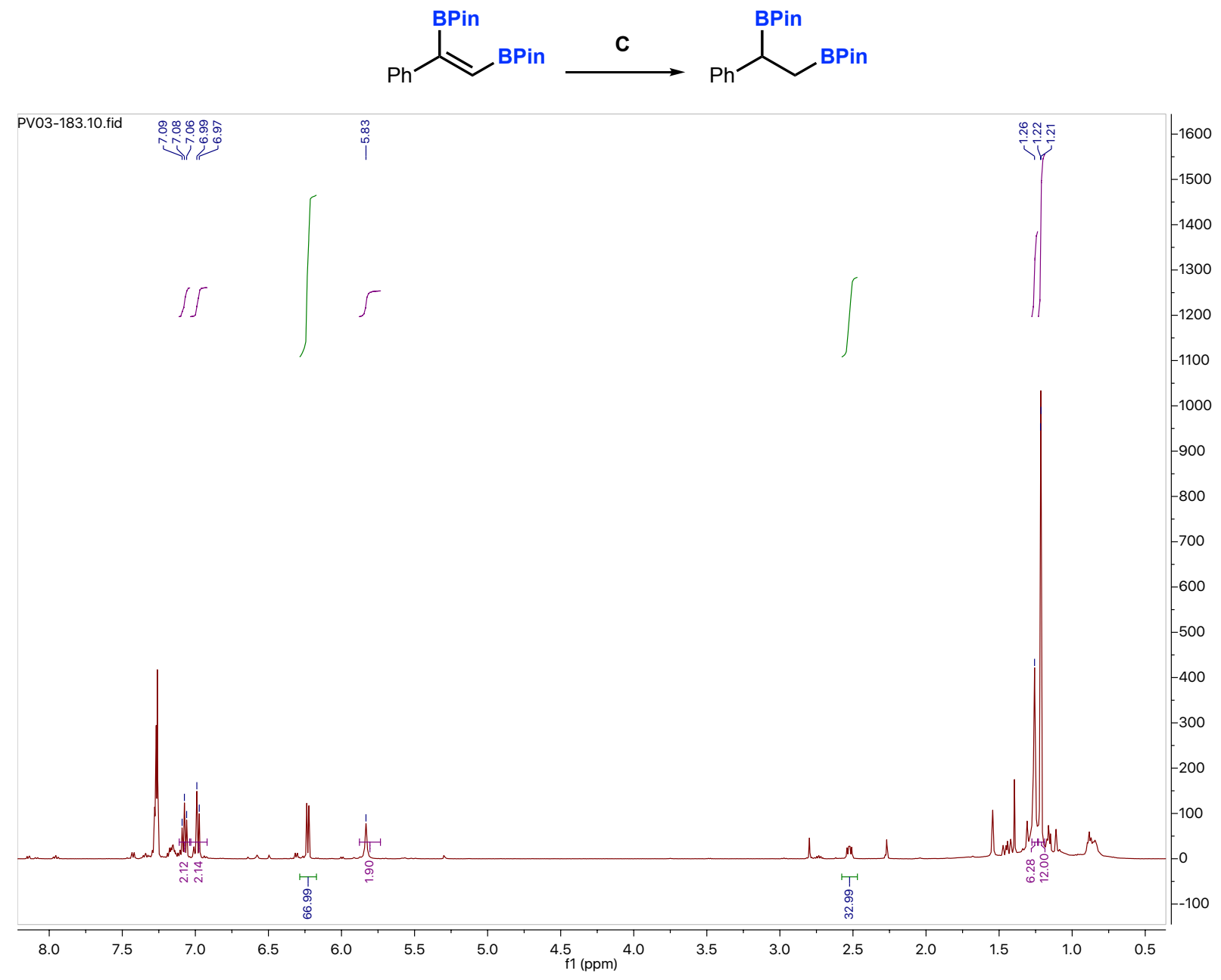

Figure S6. 1H NMR Spectrum of failed hydrogenation of 3c. Catalyst observed at $\delta=-0.49$, 1.12 indicates no catalyst death. 


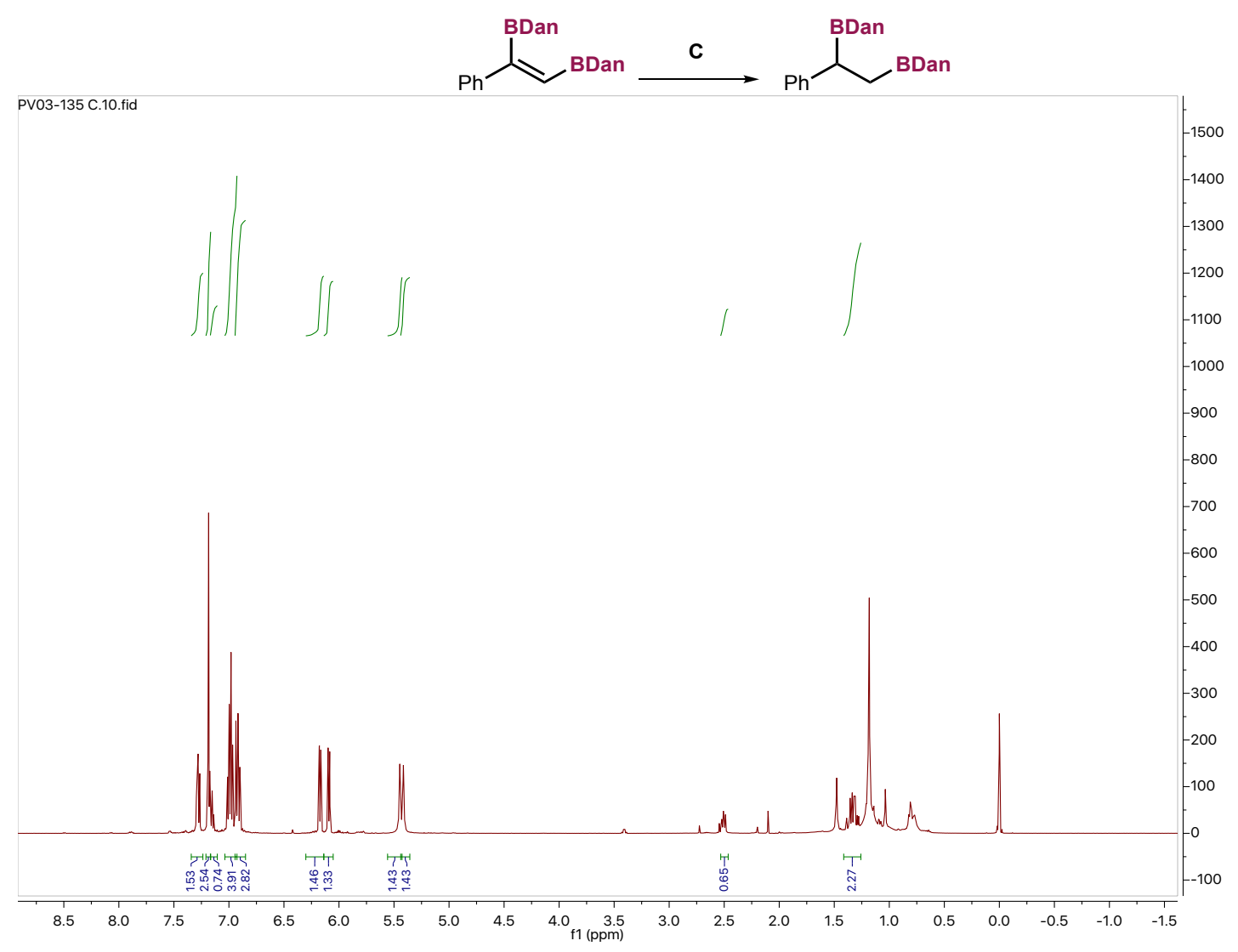

Figure S7. 1H NMR Spectrum of hydrogenation of $\mathbf{3 d}$. 


\section{Hydrogenation of Indenes}

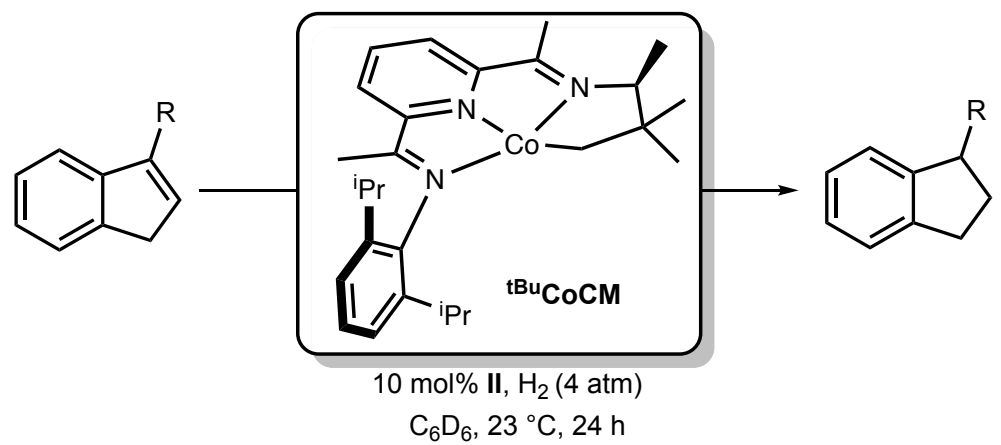

Procedure D: To a $20 \mathrm{~mL}$ was added indenyl substrate $(0.025 \mathrm{mmol})$. $\mathrm{A} \mathrm{C}_{6} \mathrm{D}_{6}$ solution $(1.4 \mathrm{~mL})$ containing ${ }^{\mathrm{tBu}} \mathrm{CoCM}(8 \mathrm{mg}, 0.0175 \mathrm{mmol})$ was prepared. To each vial was added $0.2 \mathrm{~mL}$ of this solution. The solution of each vial was transferred to a J-Young tube with a $1 \mathrm{~mL}$ syringe, and the vial was washed with benzene $(1 \times 0.2 \mathrm{~mL})$, so that the total volume of each reaction solution was $0.4 \mathrm{~mL}$. Each J-Young tube was sealed and brought out of the glovebox and connected to a high vacuum line. Each J-Young tube was frozen in liquid nitrogen, evacuated, and backfilled with 1 atm of hydrogen gas at liquid nitrogen temperature, corresponding to approximately 4 atm of hydrogen pressure at $23^{\circ} \mathrm{C}$. The frozen reaction solutions were left to thaw at the same, after which it was vertically rotated for $2 \mathrm{~h}$. The color of the reaction solution under an atmosphere of hydrogen was purple (the same shade of purple as when cobalt complex ${ }^{\mathrm{tBu}} \mathrm{CoCM}$ is dissolved in benzene). After this time, conversion was measured directly using ${ }^{1} \mathrm{H}$ NMR spectroscopy by relative integration of starting material and product. Enantioselectivities of indenyl boronates were determined after addition of a saturated $\mathrm{H}_{2} \mathrm{O}$ :THF solution of $\mathrm{NaOH}$ and $\mathrm{H}_{2} \mathrm{O}_{2}$ and treatment through a silica plug.

HPLC (Chiralcel OD-H): $40 \% \mathrm{MeOH}(0.1 \% \mathrm{DEA}) / \mathrm{CO}_{2} 100 \mathrm{bar}, 3 \mathrm{~mL} / \mathrm{min} . \mathrm{T}=3.77,4.13 \mathrm{~min}$. 


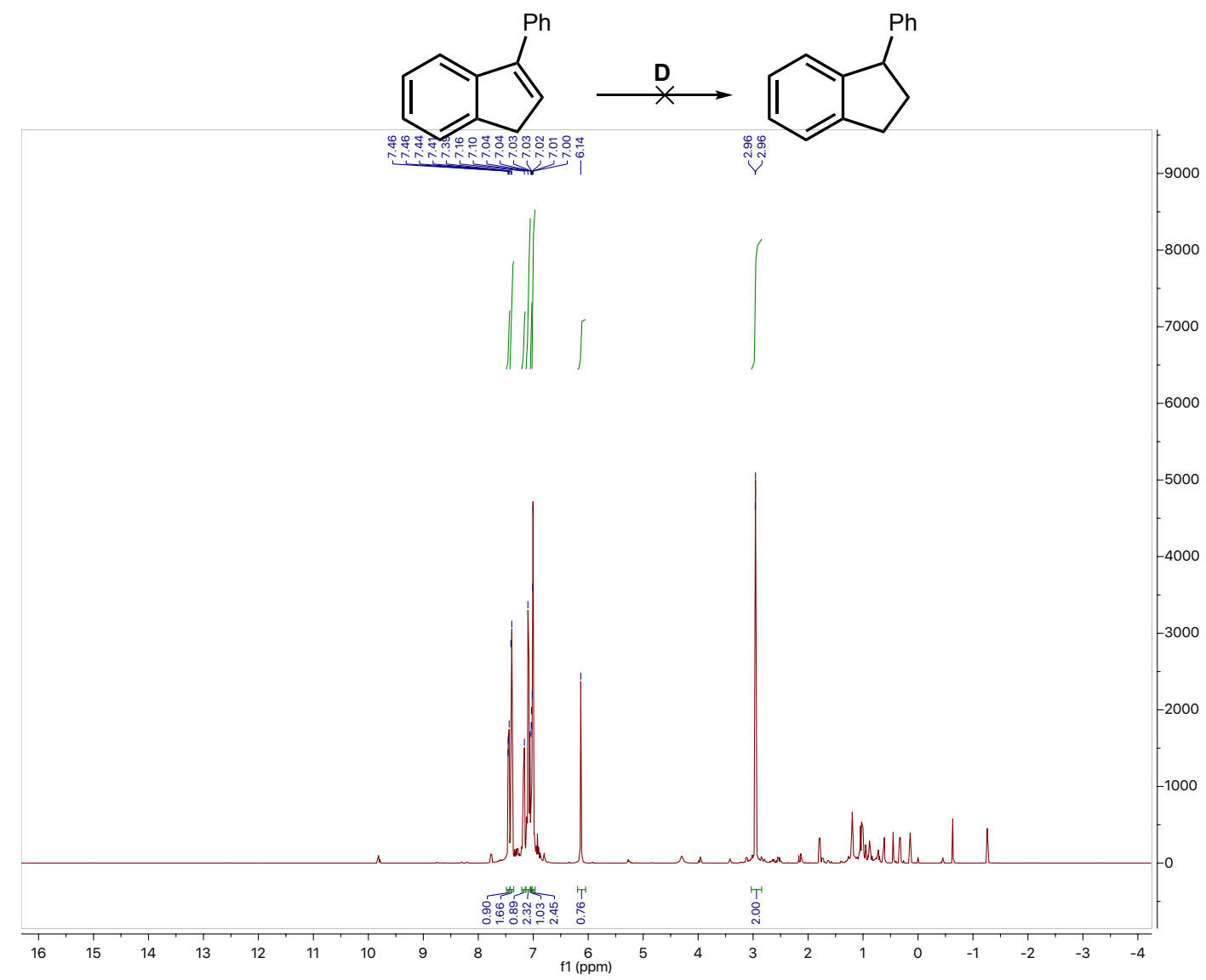

Figure S8. ${ }^{1} \mathrm{H}$ NMR spectrum of the hydrogenation of $\mathbf{5 b}$. Catalyst observed at $\delta=-0.49,-1.12$ indicates no catalyst death. 

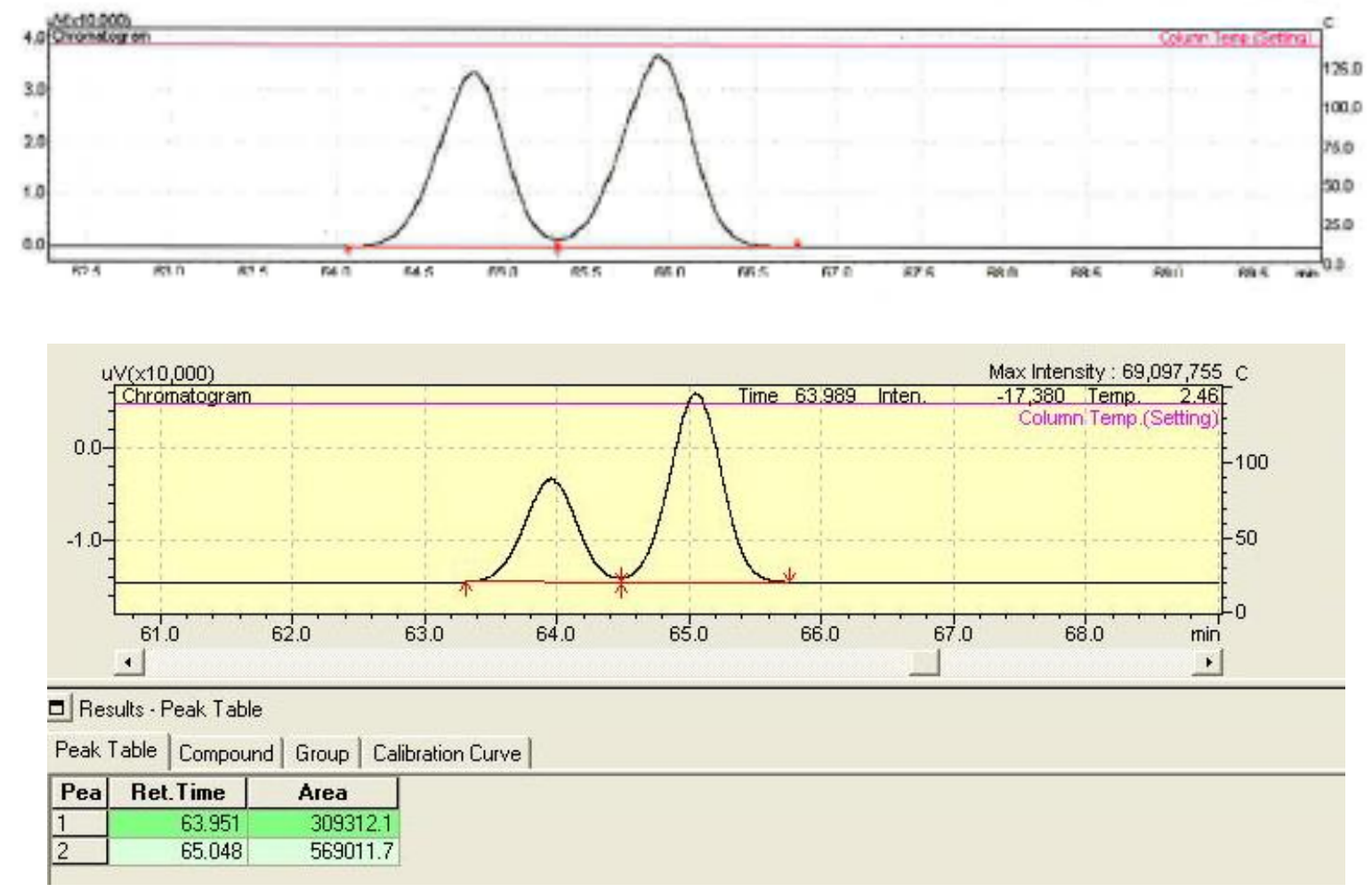

Figure 59. Racemic $\mathbf{6 b}$ (top). Enantioenriched $\mathbf{6 b}$ bottom. 


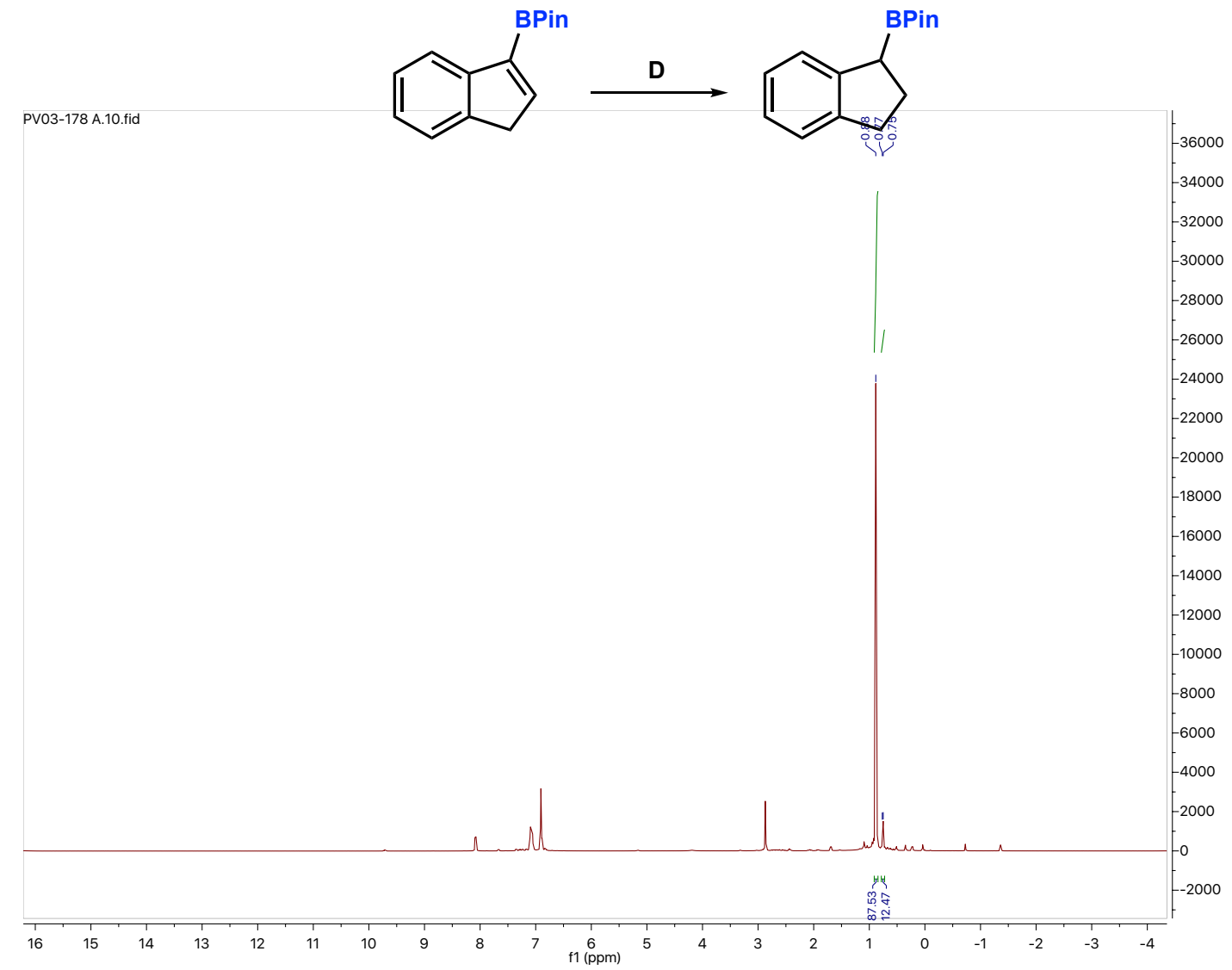

Figure S10. ${ }^{1} \mathrm{H}$ NMR spectrum of the hydrogenation of $\mathbf{5 d}$. Catalyst observed at $\delta=-0.49,-1.12$ indicates no catalyst death. 


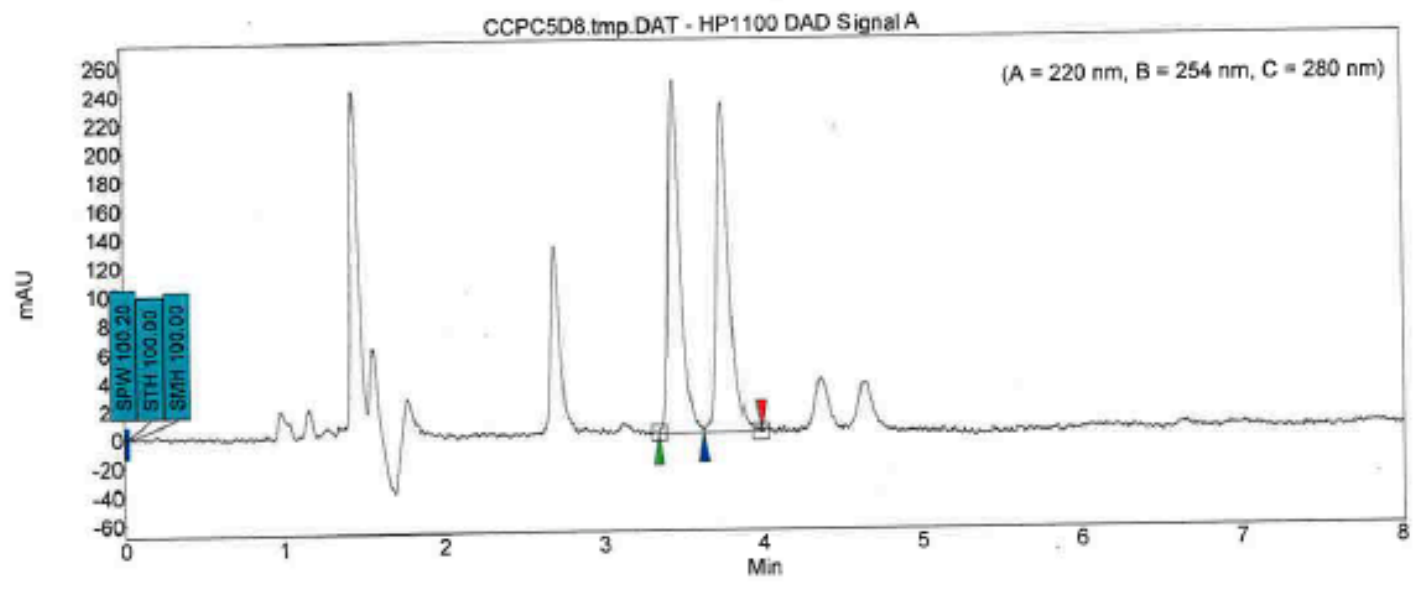

\begin{tabular}{|c|r|r|r|r|r|r|r|}
\hline Index & Time & Width & Height & Res. HW & Seloctivity & Area & Avea \\
\hline & {$[$ Min] } & {$[$ Min] } & {$[\mu \mathrm{V}]$} & & & [uV.Min] & {$[\%]$} \\
\hline 1 & 3.46 & 0.08 & 246.7 & 0.00 & 0.00 & 21.2 & 50.047 \\
\hline 2 & 3.76 & 0.08 & 230.5 & 2.26 & 1.09 & 21.1 & 49.953 \\
\hline & & & & & & & \\
\hline Total & & & 477.2 & & & 42.3 & 100.000 \\
\hline
\end{tabular}

Figure S11. Chiral SFC chromatogram of oxidized 5d. 


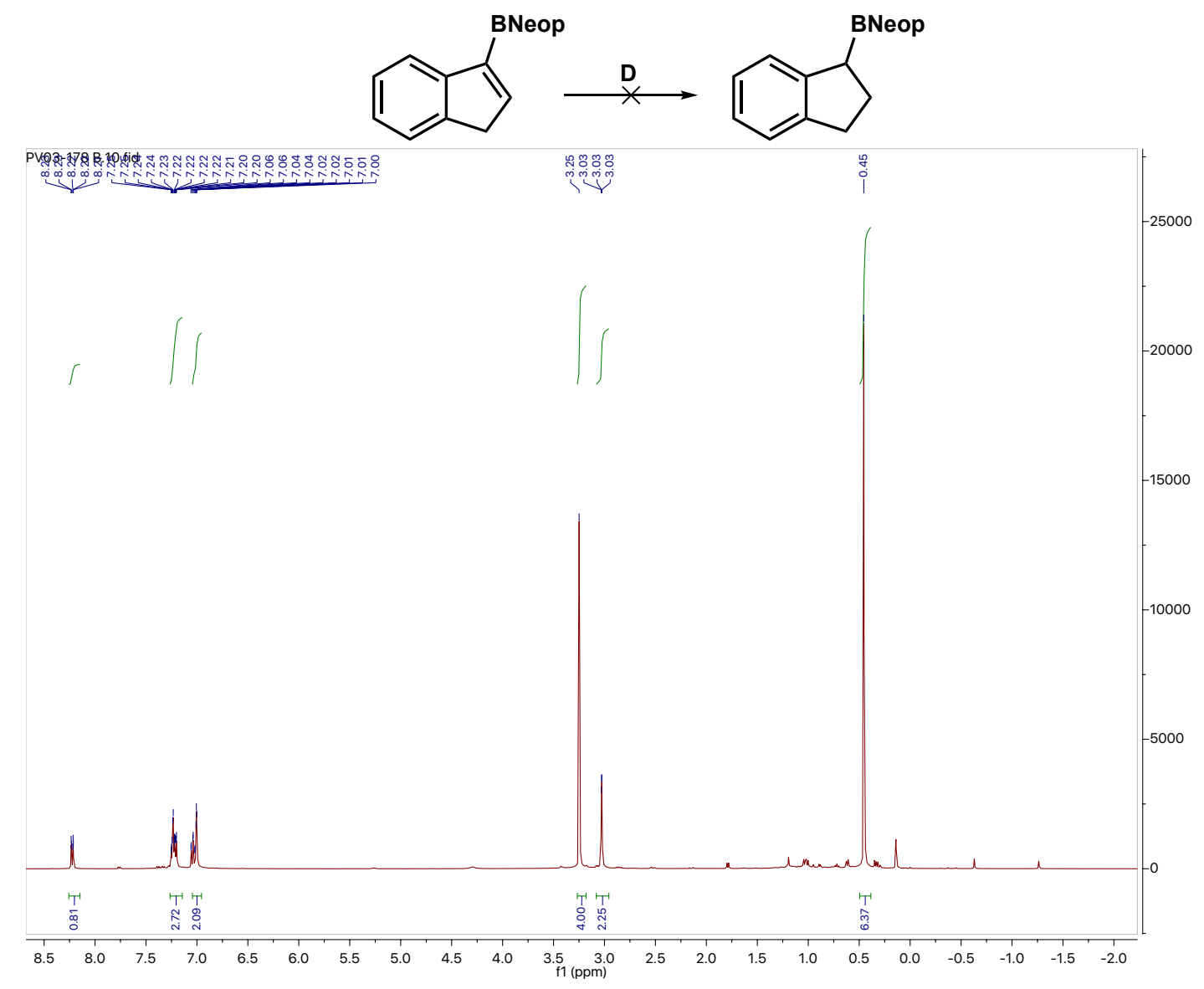

Figure S12. ${ }^{1} \mathrm{H}$ NMR spectrum of the hydrogenation of 5 c. Catalyst observed at $\delta=-0.49,-1.12$ indicates no catalyst death. 


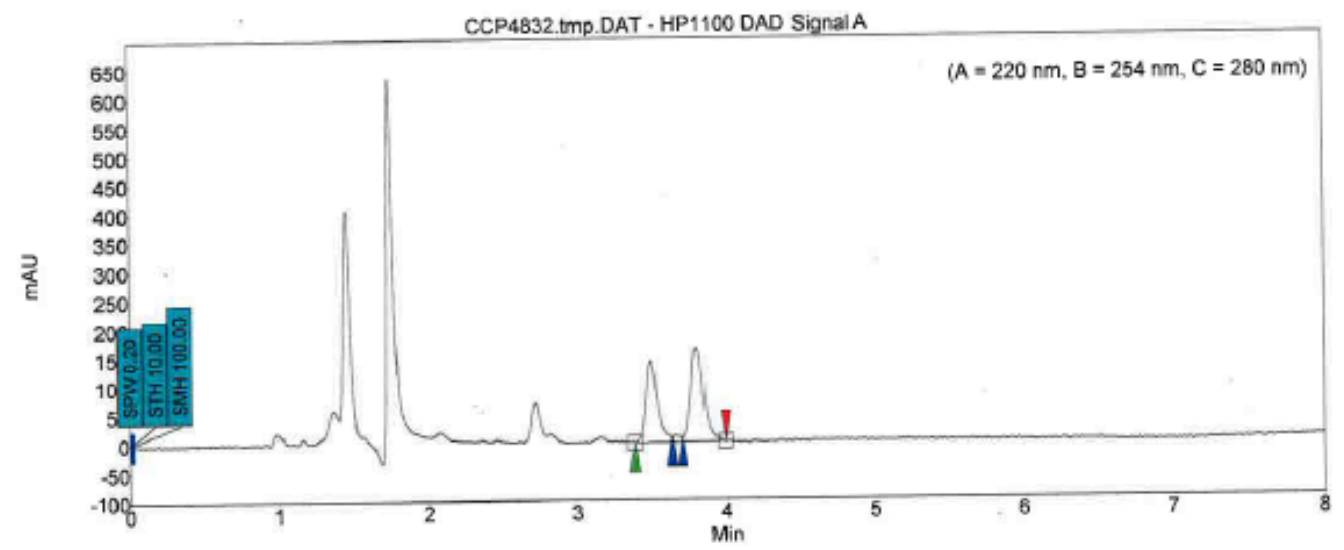

\begin{tabular}{|r|r|r|r|r|r|r|r|}
\hline Index & Time & Width & Height & Res. HW & Selectlvity & Area & Area \\
\hline \hline & {$[$ Min] } & {$[$ Min] } & {$[\mu$ ] } & & & [uV.Min] & {$[\%]$} \\
\hline 1 & 3.50 & 0.08 & 140.8 & 0.00 & 0.00 & 12.6 & 44.509 \\
\hline 2 & 3.67 & 0.07 & 11.5 & 1.31 & 1.05 & 0.7 & 2.486 \\
\hline 3 & 3.80 & 0.08 & 163.5 & 1.04 & 1.04 & 15.0 & 53.005 \\
\hline & & & & & & & \\
\hline Total & & & 315.7 & & & 28.3 & 100.000 \\
\hline
\end{tabular}

Figure S13. Chiral SFC chromatogram of oxidized 5c. 


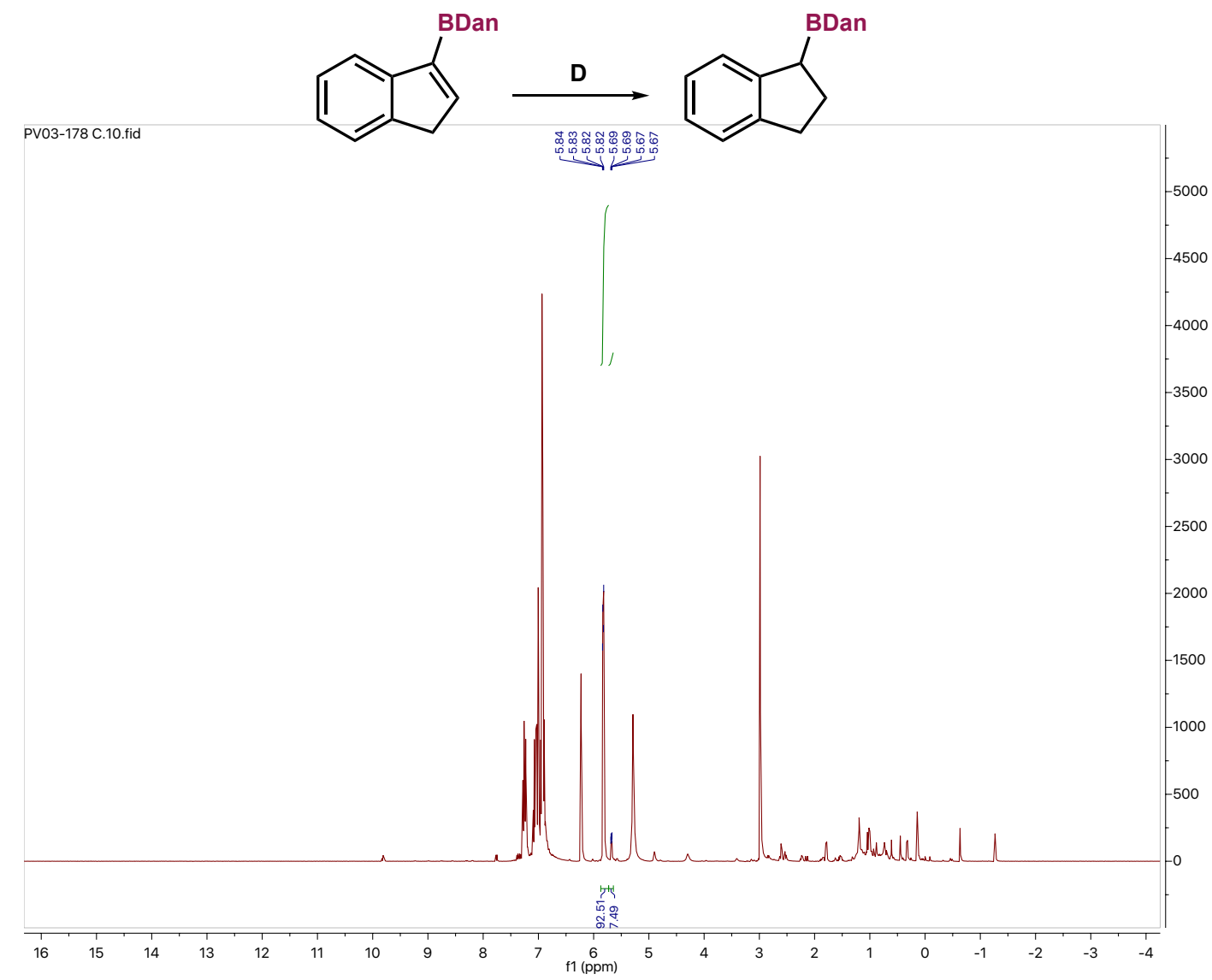

Figure S14. ${ }^{1} \mathrm{H}$ NMR spectrum of the hydrogenation of $\mathbf{5 e}$. Catalyst observed at $\delta=-0.49,-1.12$ indicates no catalyst death. 


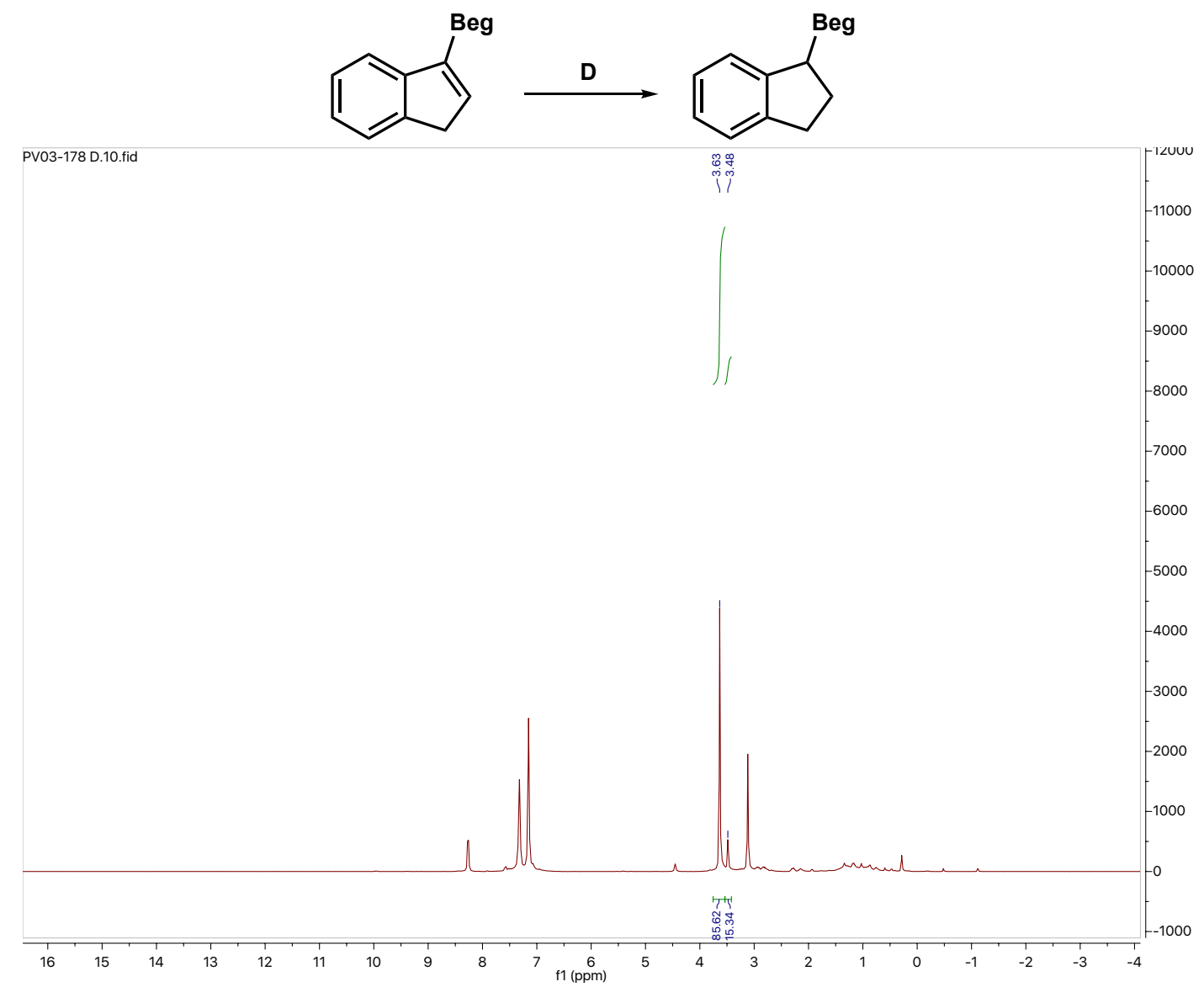

Figure S15. ${ }^{1} \mathrm{H}$ NMR spectrum of the hydrogenation of $\mathbf{5 f}$. Catalyst observed at $\delta=-0.49,-1.12$ indicates no catalyst death. 


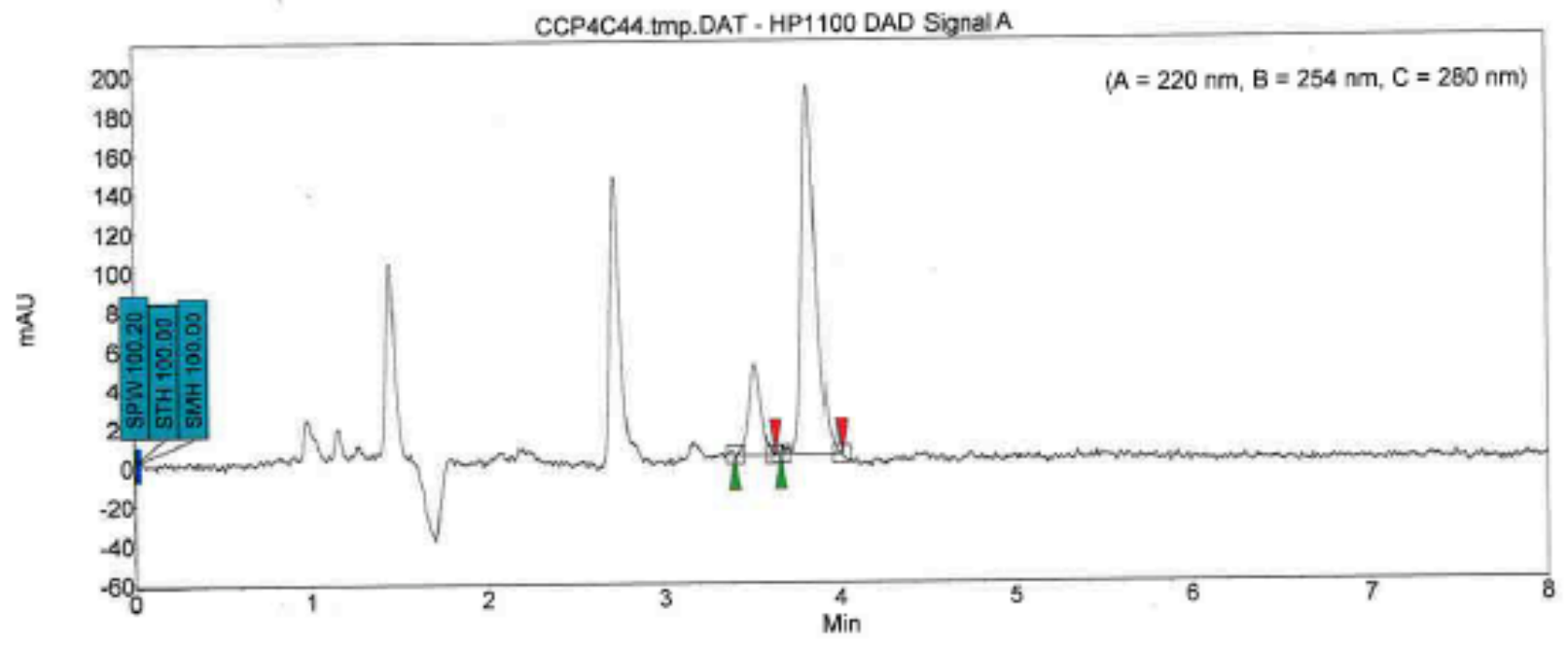

\begin{tabular}{|c|r|r|r|r|r|r|r|}
\hline Index & Time & Width & Height & Res. HW & Selectivity & Areg & Area \\
\hline & {$[$ Min] } & {$[$ Min] } & {$[\mu \mathrm{M}]$} & & & {$[\mu$ V.Min] } & {$[\%]$} \\
\hline 1 & 3.52 & 0.08 & 46.6 & 0.00 & 0.00 & 3.8 & 18.058 \\
\hline 2 & 3.83 & 0.08 & 188.7 & 2.36 & 1.09 & 17.3 & 81.942 \\
\hline & & & & & & & \\
\hline Total & & & 235.3 & & & 21.1 & 100.000 \\
\hline
\end{tabular}

Figure S16. Chiral SFC chromatogram of oxidized $\mathbf{5 f .}$ 


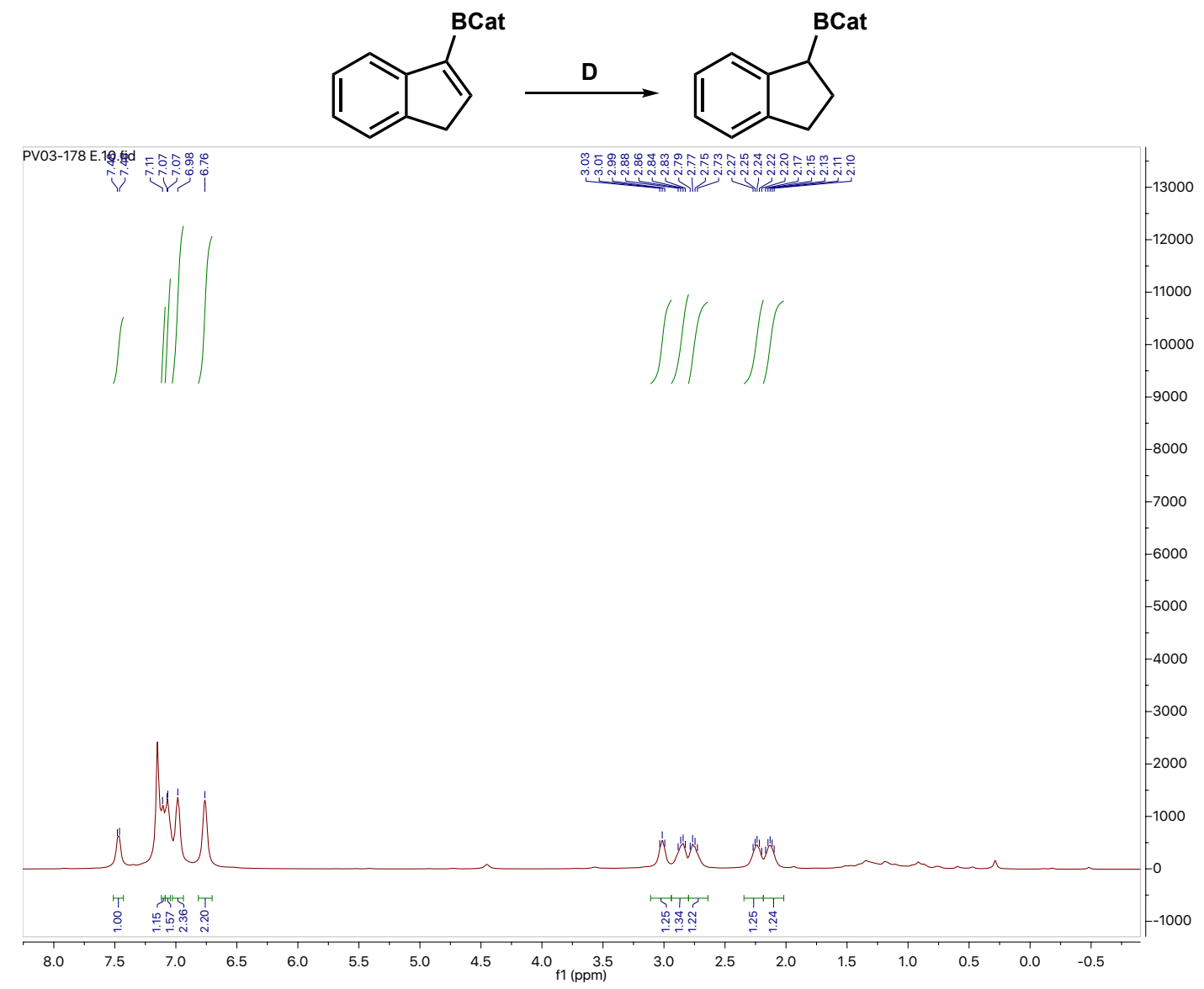

Figure S17. ${ }^{1} \mathrm{H}$ NMR spectrum of the hydrogenation of $\mathbf{5 g}$. No starting material is detected. 


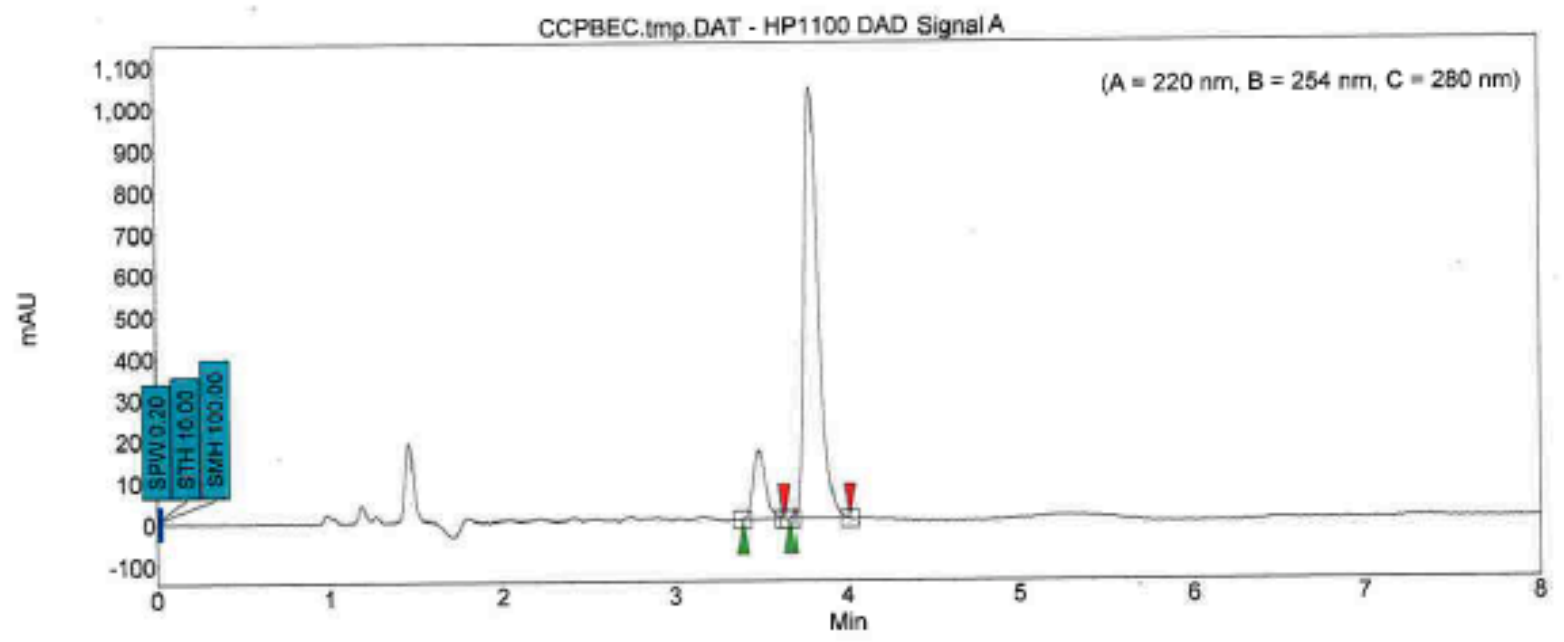

\begin{tabular}{|c|r|r|r|r|r|r|r|}
\hline Index & Time & Width & Height & Res. HW & Selectivity & Area & Area \\
\hline \hline & [Min] & {$[$ Min] } & {$[\mu \mathrm{M}$} & & & [uV.Min] & {$[\%]$} \\
\hline 1 & 3.49 & 0.07 & 162.7 & 0.00 & 0.00 & 13.3 & 12.128 \\
\hline 2 & 3.78 & 0.08 & 1038.2 & 2.20 & 1.08 & 96.1 & 87.872 \\
\hline & & & & & & & \\
\hline Total & & & 1200.9 & & & 109.3 & 100.000 \\
\hline
\end{tabular}

Figure S18. Chiral SFC chromatogram of oxidized $\mathbf{5 g}$. 


\section{Deuterium Labeling Studies}

Deuteration and Oxidation of Indanyl boronates: To a $20 \mathrm{~mL}$ vial containing cobalt complex ${ }^{\mathrm{tBu}} \mathrm{CoCM}(4.63 \mathrm{mg}, 10 \mathrm{~mol} \%)$ was added indenyl boronate $(0.10 \mathrm{mmol})$ and benzene $(1.0 \mathrm{~mL})$. The solution was transferred to a thick walled glass bomb with a glass pipette, and the vial was washed with benzene $(1 \times 0.5 \mathrm{~mL})$, so that the total volume of the reaction solution was $1.5 \mathrm{~mL}$. A magnetic stir bar was added, and the bomb was sealed with a Kontes valve. The vessel was brought out of the glovebox and connected to a high vacuum line. The vessel was frozen in liquid nitrogen, evacuated, and backfilled with 1 atm of hydrogen gas at liquid nitrogen temperature, corresponding to approximately $4 \mathrm{~atm}$ of hydrogen pressure at $23^{\circ} \mathrm{C}$. The frozen reaction solution was then left to thaw, after which it was magnetically stirred for the period of time indicated. The color of the reaction solution under an atmosphere of hydrogen was purple (the same shade of purple as when cobalt complex ${ }^{\mathrm{tBu}} \mathrm{CoCM}$ is dissolved in benzene). The vessel was then opened to air, and a freshly prepared solution of $\mathrm{NaOH}(20 \mathrm{mg})$ and $\mathrm{H}_{2} \mathrm{O}_{2}(0.1$ $\mathrm{mL}, 30 \%$ aq.) in THF: $\mathrm{H}_{2} \mathrm{O}$ was added directly into the solution, stirred for 2 hours at room temperature, extracted with DCM $(3 \times 5 \mathrm{~mL})$, the solvent removed, and purified by silica gel column chromatography hexanes: ethyl acetate 10:1. 

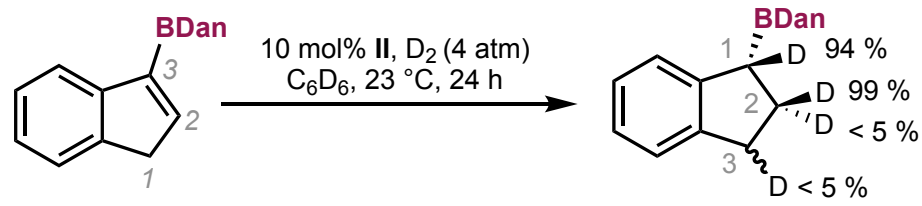

$60 \%$ ee

Natural Abundance Spectra (For reference):

${ }^{1} \mathrm{H}$ NMR $(500 \mathrm{MHz}$, Chloroform-d) $\delta 7.29$ (dd, $J=5.7,2.6 \mathrm{~Hz}, 1 \mathrm{H}), 7.24-7.19(\mathrm{~m}, 1 \mathrm{H}), 7.19-$ $7.16(\mathrm{~m}, 2 \mathrm{H}), 7.09(\mathrm{dd}, J=8.3,7.3 \mathrm{~Hz}, 2 \mathrm{H}), 7.01(\mathrm{dd}, J=8.3,1.0 \mathrm{~Hz}, 2 \mathrm{H}), 6.27(\mathrm{dd}, J=7.3,1.1$ $\mathrm{Hz}, 2 \mathrm{H}), 5.59$ (s, 2H), $2.97(\mathrm{t}, J=7.4 \mathrm{~Hz}, 2 \mathrm{H}), 2.80(\mathrm{t}, J=8.1 \mathrm{~Hz}, 1 \mathrm{H}), 2.37$ (ddt, $J=12.3,9.0$, $7.1 \mathrm{~Hz}, 1 \mathrm{H}), 2.05(\mathrm{dq}, J=12.5,7.6 \mathrm{~Hz}, 1 \mathrm{H})$.

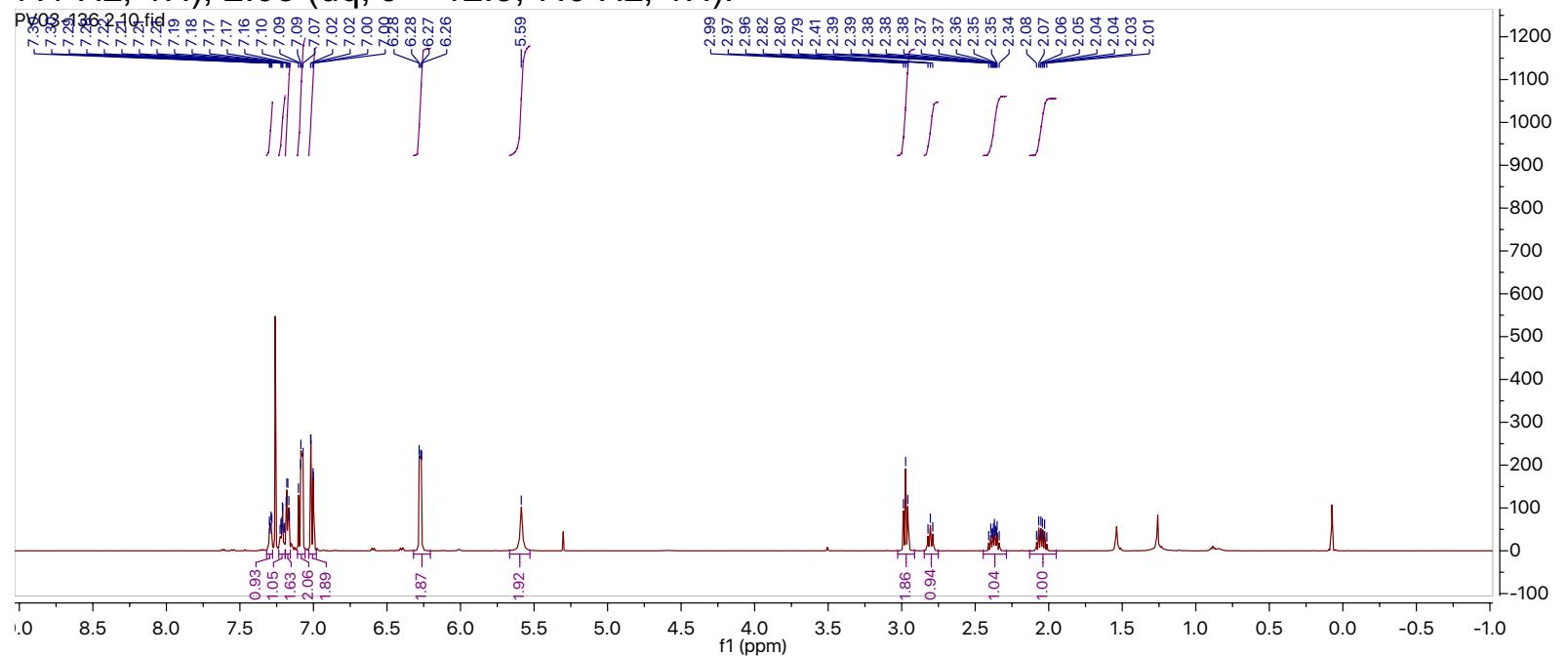

Figure S19. ${ }^{1} \mathrm{H}$ NMR spectrum of the natural abundance $5 \mathbf{e}$.

${ }^{13} \mathrm{C}$ NMR $\left(126 \mathrm{MHz}, \mathrm{CDCl}_{3}\right) \delta 145.76,144.51,141.04,136.31,127.55,126.47,125.89,124.66$, 124.23, 117.63, 105.75, 33.20.

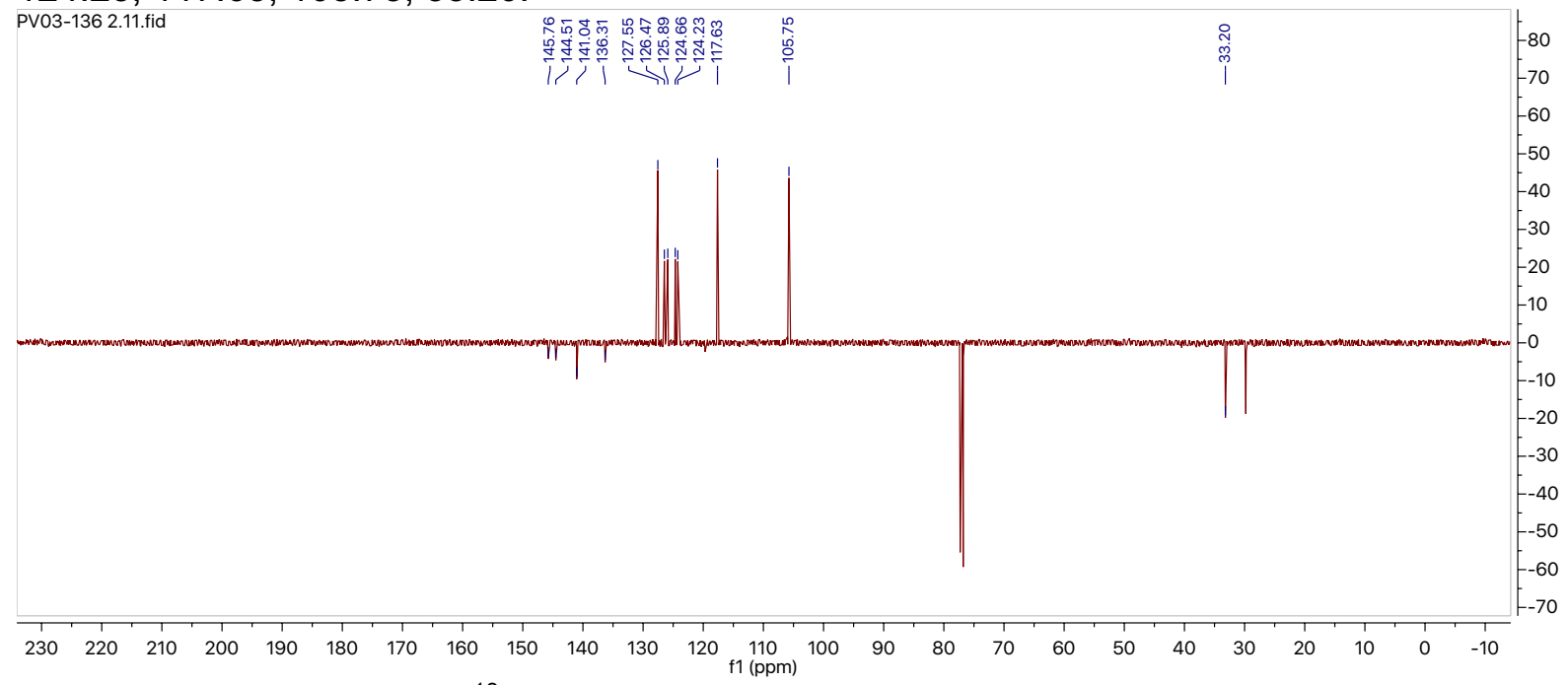

Figure S20. ${ }^{13} \mathrm{C}(\mathrm{APT}) \mathrm{NMR}$ spectrum of the natural abundance $\mathbf{5 e}$. 
Determination of $\mathrm{D}$-incorporation:

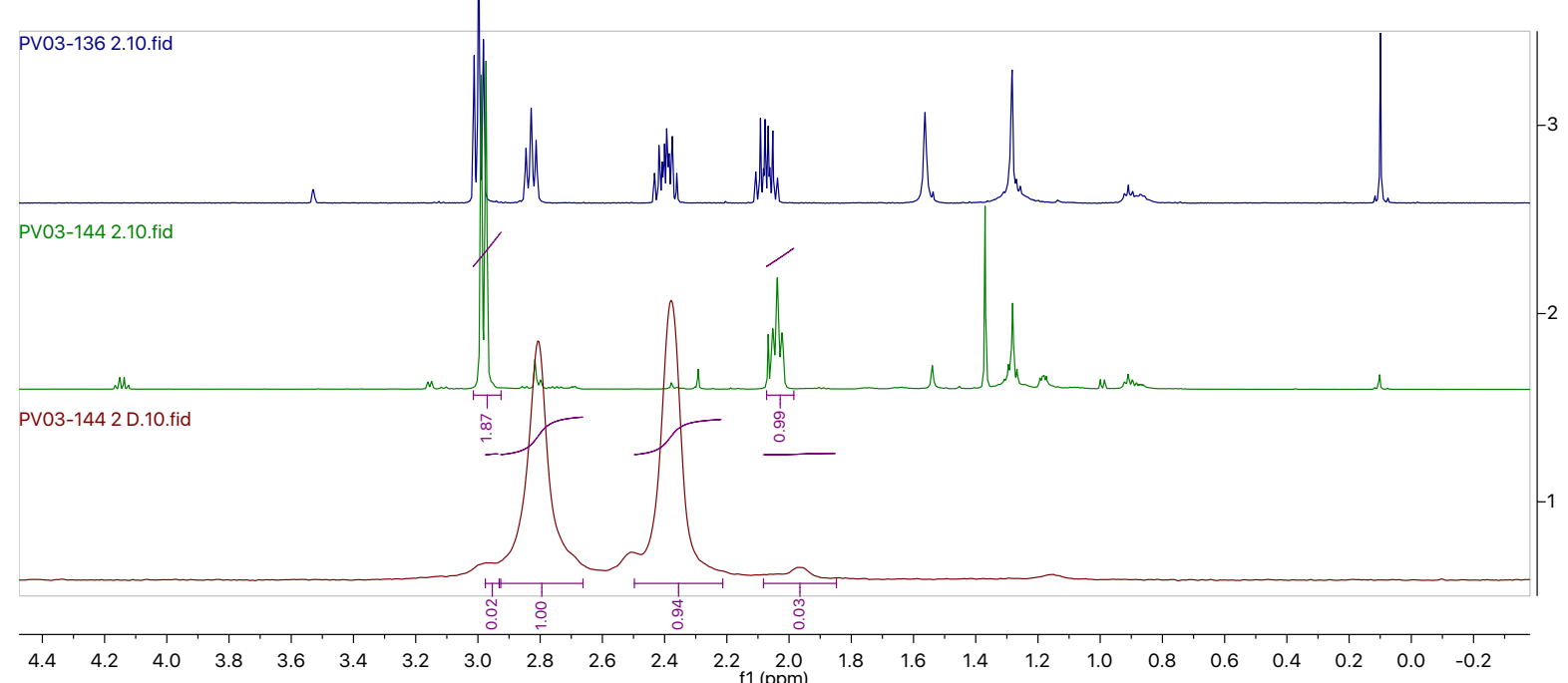

Figure S21. ${ }^{1} \mathrm{H}$ NMR spectra of natural abundance compound (top). ${ }^{1} \mathrm{H}$ NMR spectra of

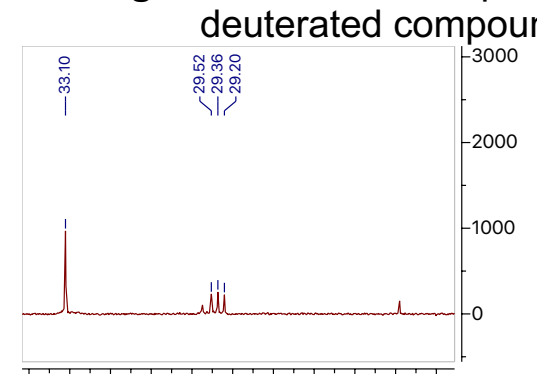

$\begin{array}{llllllllllllll}34 & 33 & 32 & 31 & 30 & 29 & 28 & 27 & 26 & 25 & 24\end{array}$

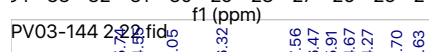

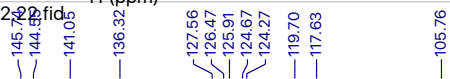
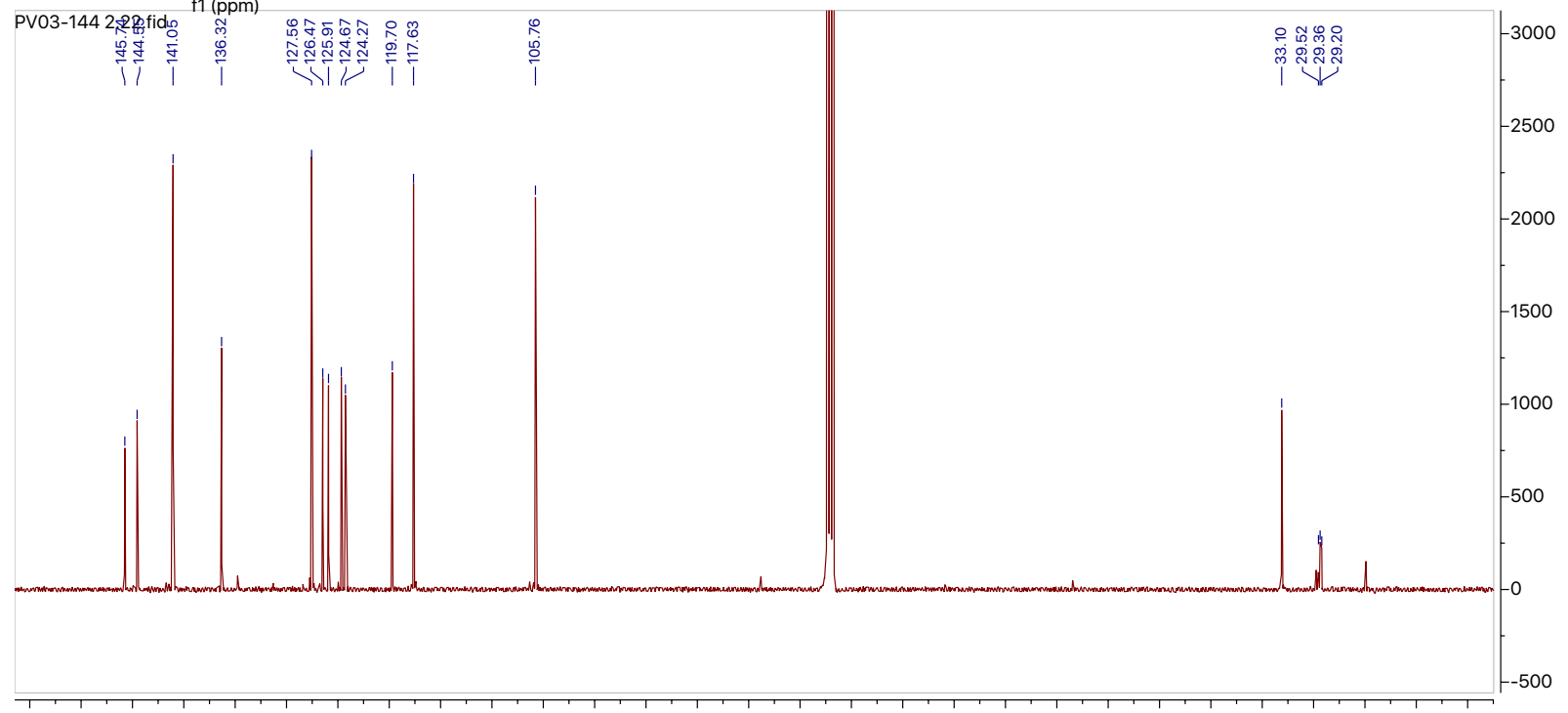

$\begin{array}{llllllllllllllllllllllllllllllllllll}155 & 150 & 145 & 140 & 135 & 130 & 125 & 120 & 115 & 110 & 105 & 100 & 95 & 90 & 85 & 80 & 75 & 70 & 65 & 60 & 55 & 50 & 45 & 40 & 35 & 30 & 25 & 20 & 15\end{array}$

Figure S22. Quant. ${ }^{13} \mathrm{C}$ of deuterated compound. ${ }^{13} \mathrm{C}$ signals $\alpha$ to boron are not observed.

HRMS (ESI+): $m / z$ calculated $\mathrm{C}_{19} \mathrm{H}_{16} \mathrm{BN}_{2}{ }^{+}\left([\mathrm{MH}]^{+}\right): 287.1683$; found: 287.1685 


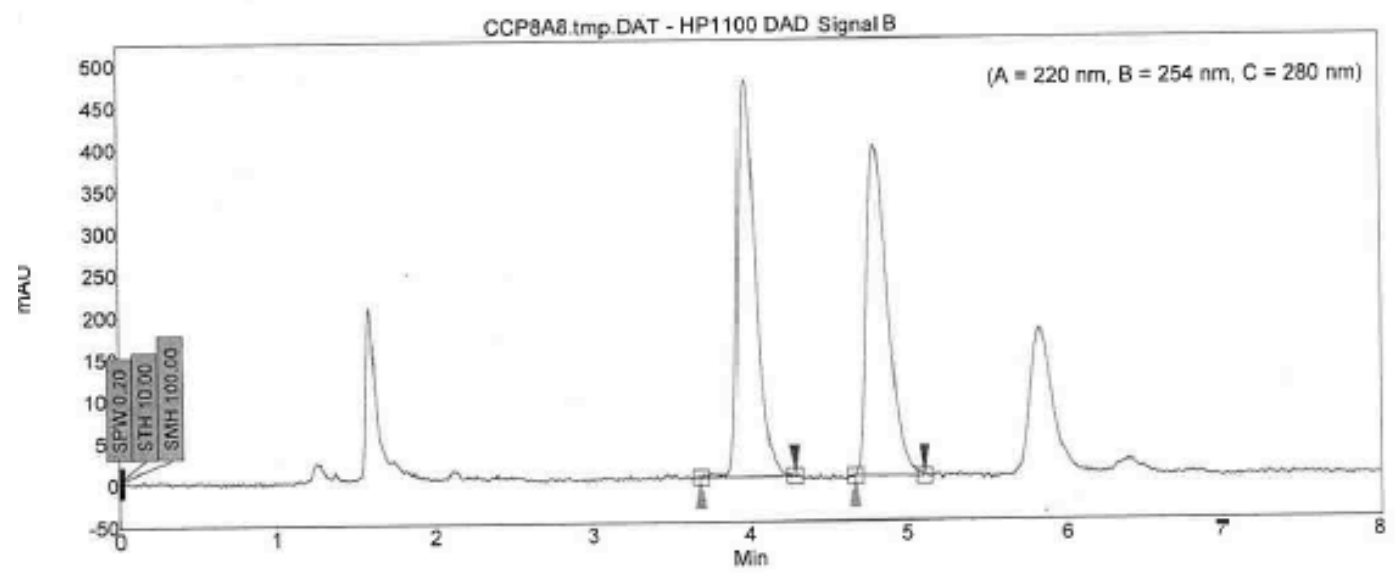

\begin{tabular}{|c|r|r|r|r|r|r|r|}
\hline Index & Time & Width & Height & Res. HW & Selectivity & Arca & Avea \\
\hline & {$[$ Min] } & {$[$ Min] } & {$[\mu \mathrm{V}]$} & & & {$[\mu \mathrm{V}$. Min] } & {$[\%]$} \\
\hline 2 & 3.97 & 0.11 & 474.0 & 0.00 & 0.00 & 59.4 & 49.488 \\
\hline 1 & 4.79 & 0.14 & 395.4 & 3.78 & 1.21 & 60.6 & 50.512 \\
\hline & & & & & & & \\
\hline Total & & & 869.4 & & & 119.9 & 100.000 \\
\hline
\end{tabular}

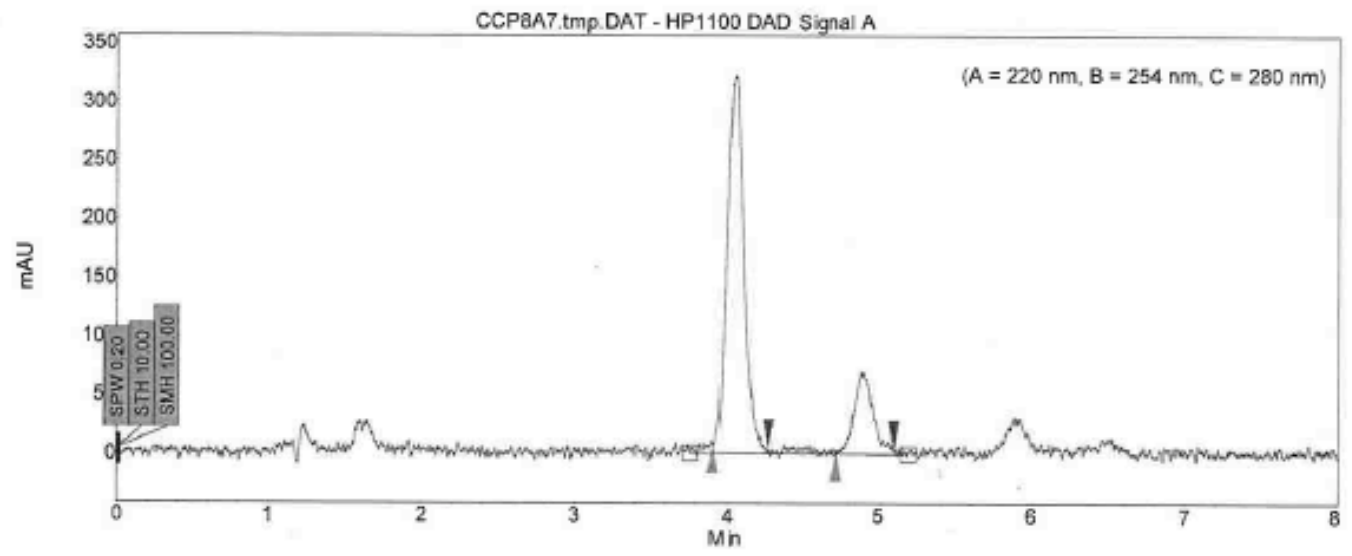

\begin{tabular}{|c|r|r|r|r|r|r|r|}
\hline Index & Timo & Width & Height & Res. HW & Selectivity & Area & Area \\
\hline & [Min] & [Min] & {$[u$ ] } & & & [UV.Min] & {$[\%]$} \\
\hline 1 & 4.04 & 0.12 & 321.1 & 0.00 & 0.00 & 40.6 & 80.277 \\
\hline 2 & 4.89 & 0.12 & 68.6 & 4.14 & 1.21 & 10.0 & 19.723 \\
\hline & & & & & & & \\
\hline Total & & & 389.7 & & & 50.6 & 100.000 \\
\hline
\end{tabular}

Figure S23. Chiral SFC Chromatogram of racemic (top) and enantioenriched (bottom) 7e-d. 

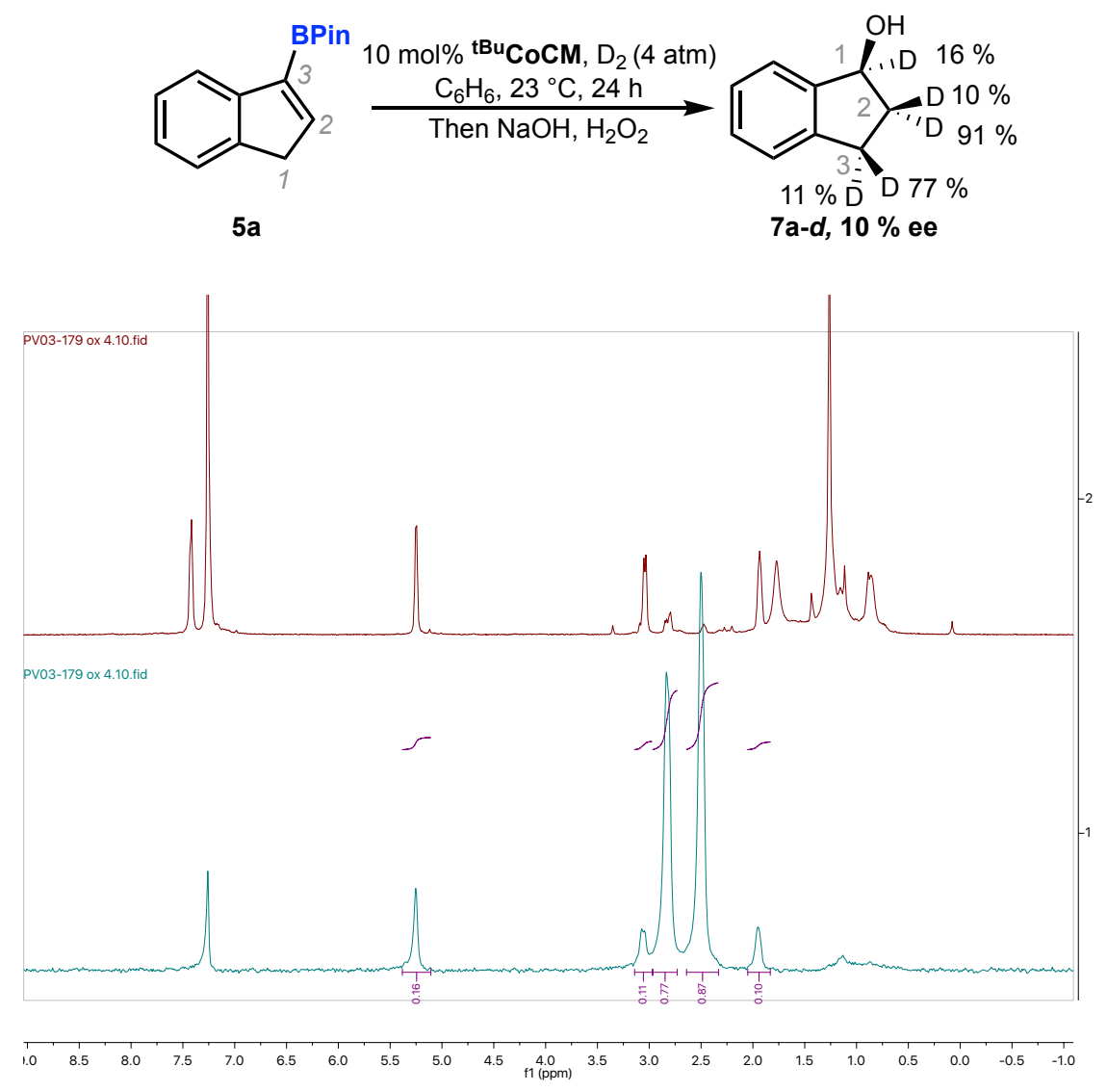

Figure S24. ${ }^{1} \mathrm{H}$ NMR of deuterated 1-indanol (top). ${ }^{2} \mathrm{H}$ NMR of deuterated 1-indanol (bottom).

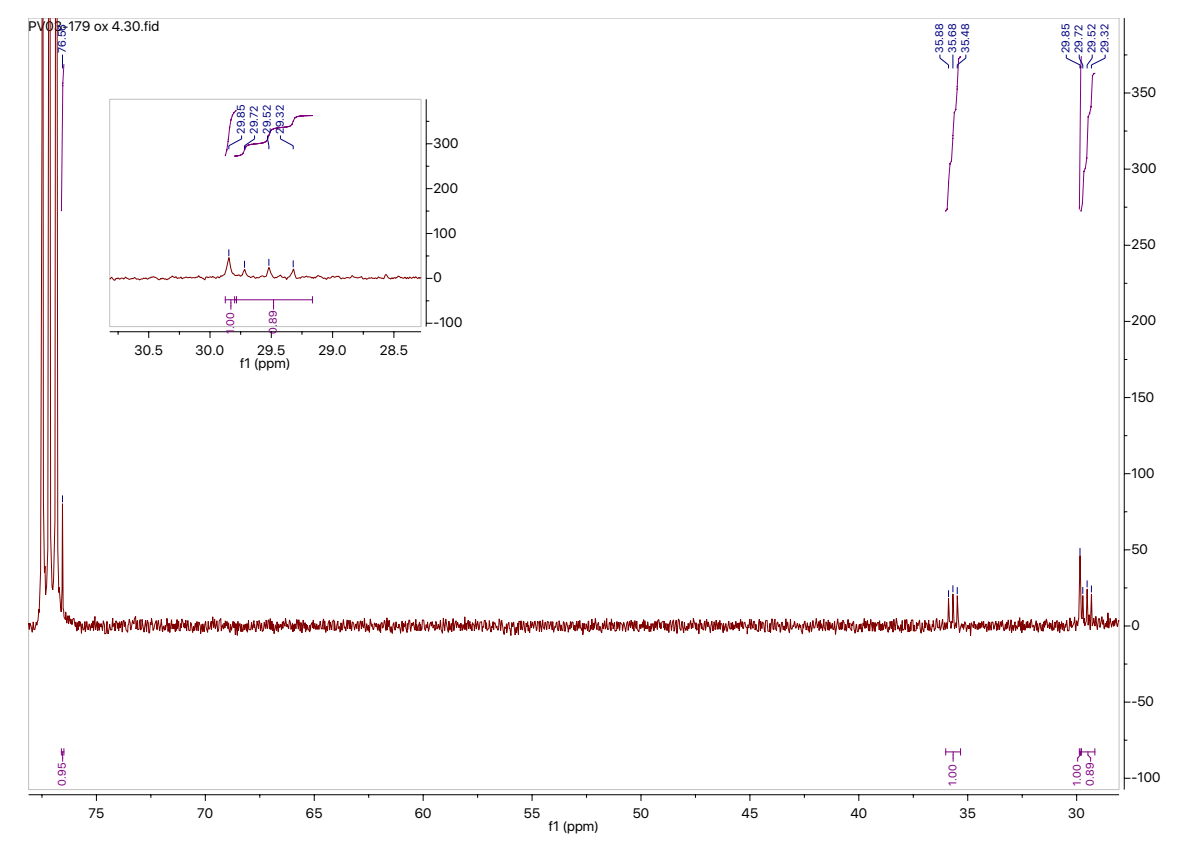

Figure S25. Quant. ${ }^{13} \mathrm{C}$ of deuterated 1-indanol. $5 \%$ un-deuterated C-1 observed. Mixtures of isotopologues led to low signal to noise for carbons bearing small incorporations of $2 \mathrm{H}$. 
HRMS (EI+):
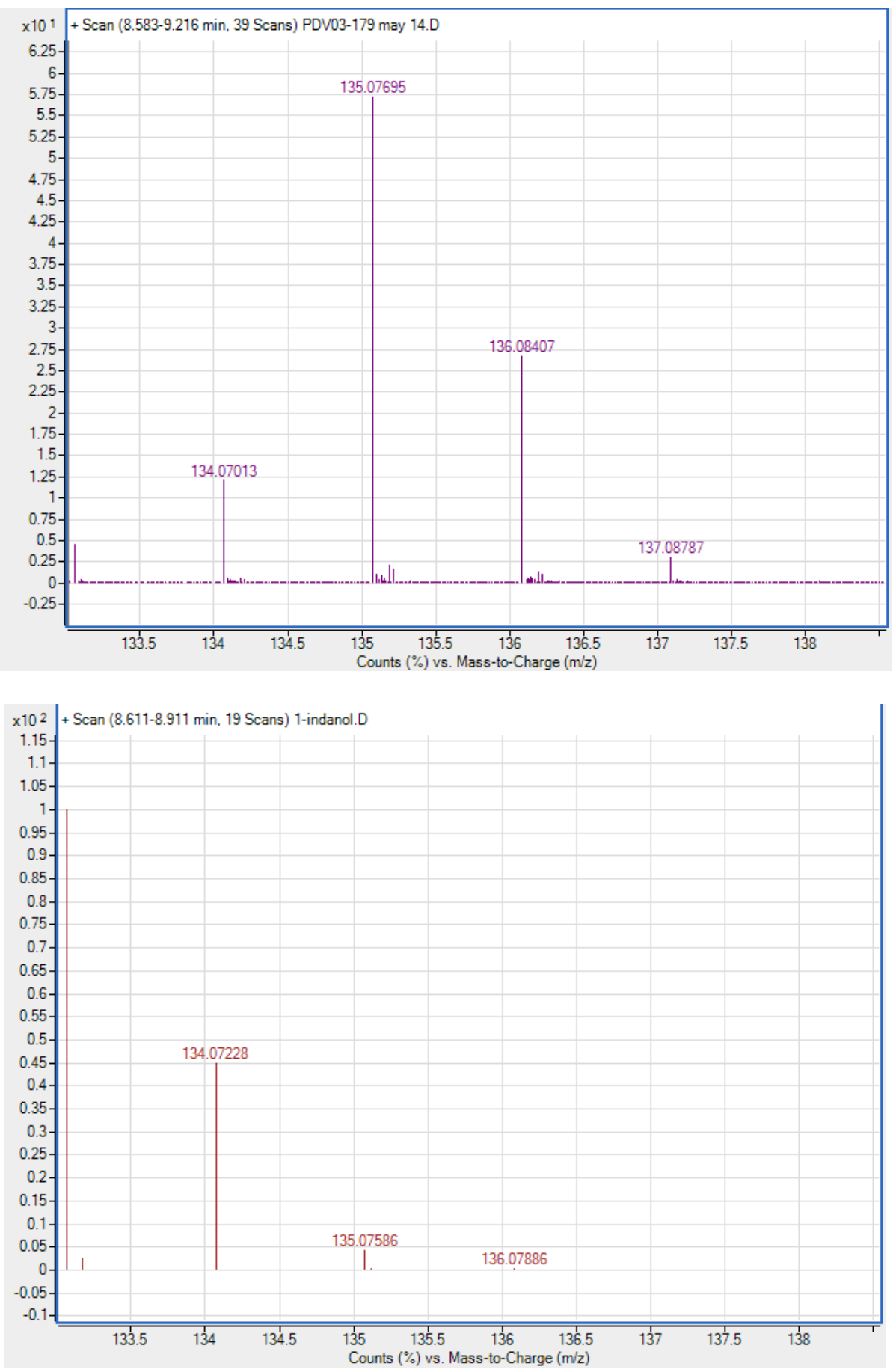

Figure S26. Deuterated indanol 7a-d (top). Natural Abundance (bottom). 


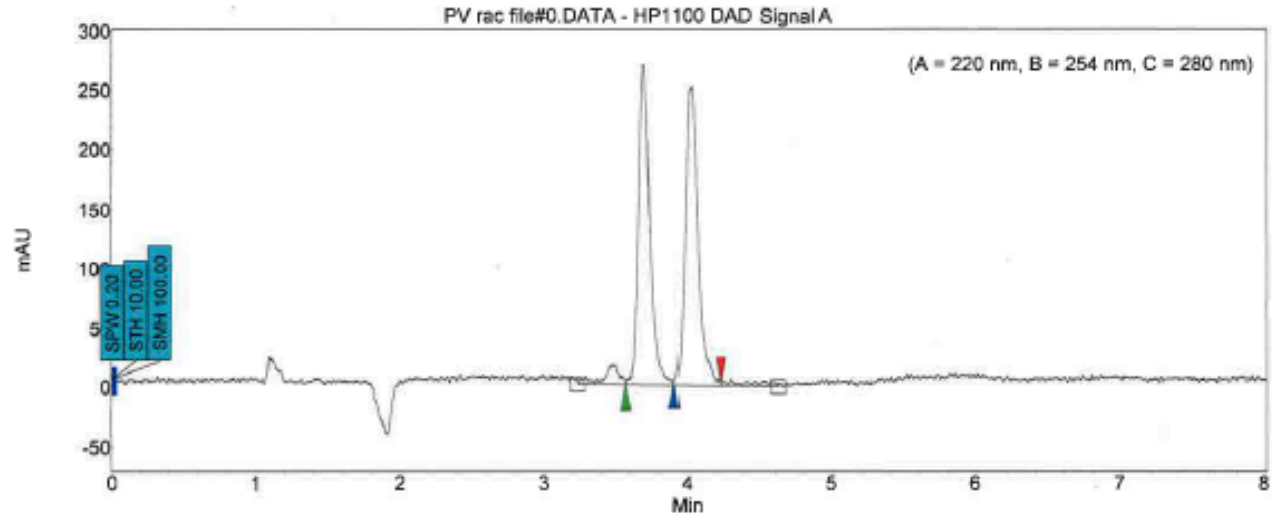

\begin{tabular}{|c|r|r|r|r|r|r|r|}
\hline Index & Time & Width & Height & Res. HW & Selectivity & Area & Area \\
\hline \hline & {$[$ Min] } & {$[$ Min] } & {$[\mu \mathrm{M}]$} & & & [uV.Min] & {$[\%]$} \\
\hline 1 & 3.69 & 0.08 & 269.5 & 0.00 & 0.00 & 24.9 & 49.805 \\
\hline 2 & 4.02 & 0.09 & 251.6 & 2.28 & 1.09 & 25.1 & 50.195 \\
\hline & & & & & & & \\
\hline Total & & & 521,1 & & & 50.0 & 100.000 \\
\hline
\end{tabular}

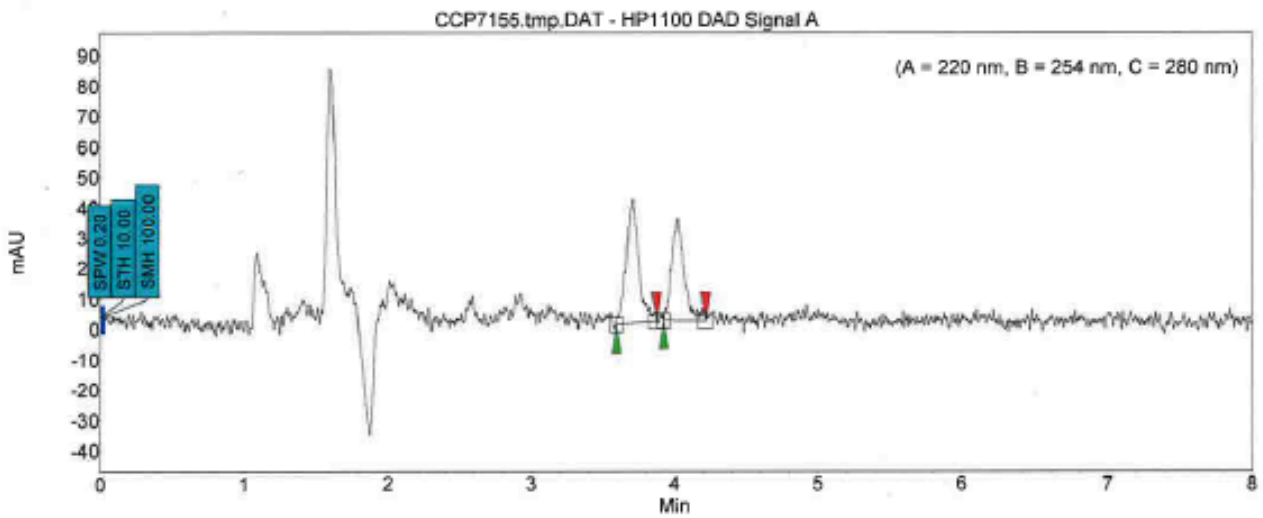

\begin{tabular}{|c|r|r|r|r|r|r|r|}
\hline Index & Time & Width & Height & Res. HW & Selectivity & Area & Area \\
\hline & {$[\mathrm{Min}]$} & {$[$ Min] } & {$[\mu \mathrm{V}]$} & & & {$[\mu \mathrm{V} . \mathrm{Min}]$} & {$[\%]$} \\
\hline 1 & 3.71 & 0.08 & 40.8 & 0.00 & 0.00 & 4.0 & 55.048 \\
\hline 2 & 4.03 & 0.08 & 33.6 & 2.28 & 1.08 & 3.3 & 44.952 \\
\hline & & & & & & & \\
\hline Total & & & 74.4 & & & 7.3 & 100.000 \\
\hline
\end{tabular}

Figure S27. SFC Chromatogram of racemic (top) and enantioenriched 7a-d. 


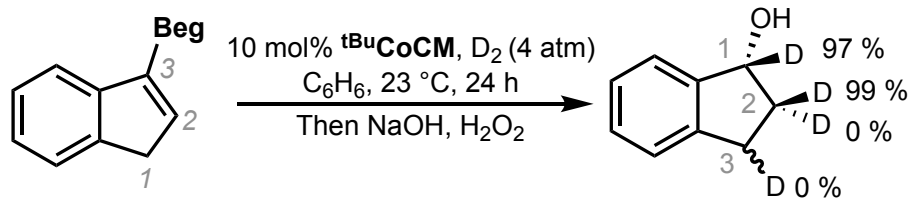

$5 f$

$7 f-d, 99 \%$ ee

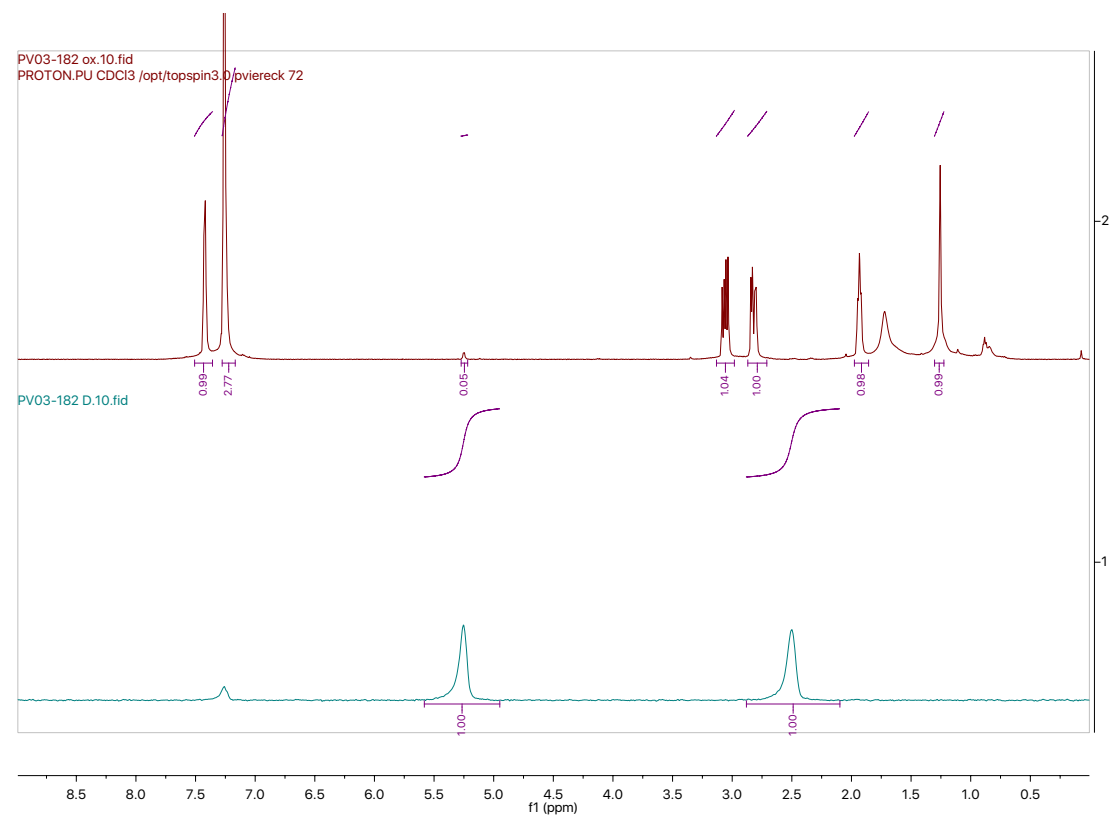

Figure S28. ${ }^{1} \mathrm{H}$ NMR of deuterated 1 -indanol (top). ${ }^{2} \mathrm{H}$ NMR of deuterated 1-indanol (bottom).

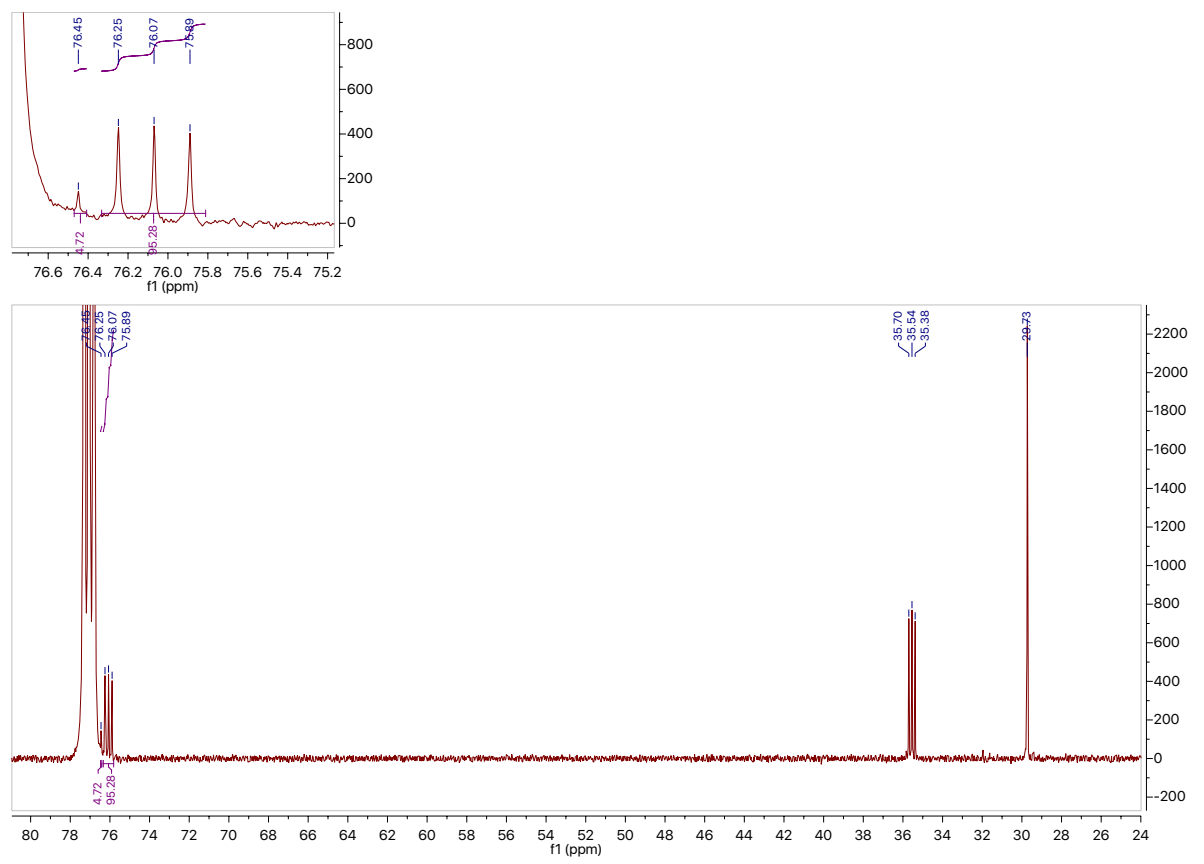

Figure S29. Quant. ${ }^{13} \mathrm{C}$ of deuterated 1-indanol. $5 \%$ un-deuterated C-1 observed. 
HRMS (EI+):
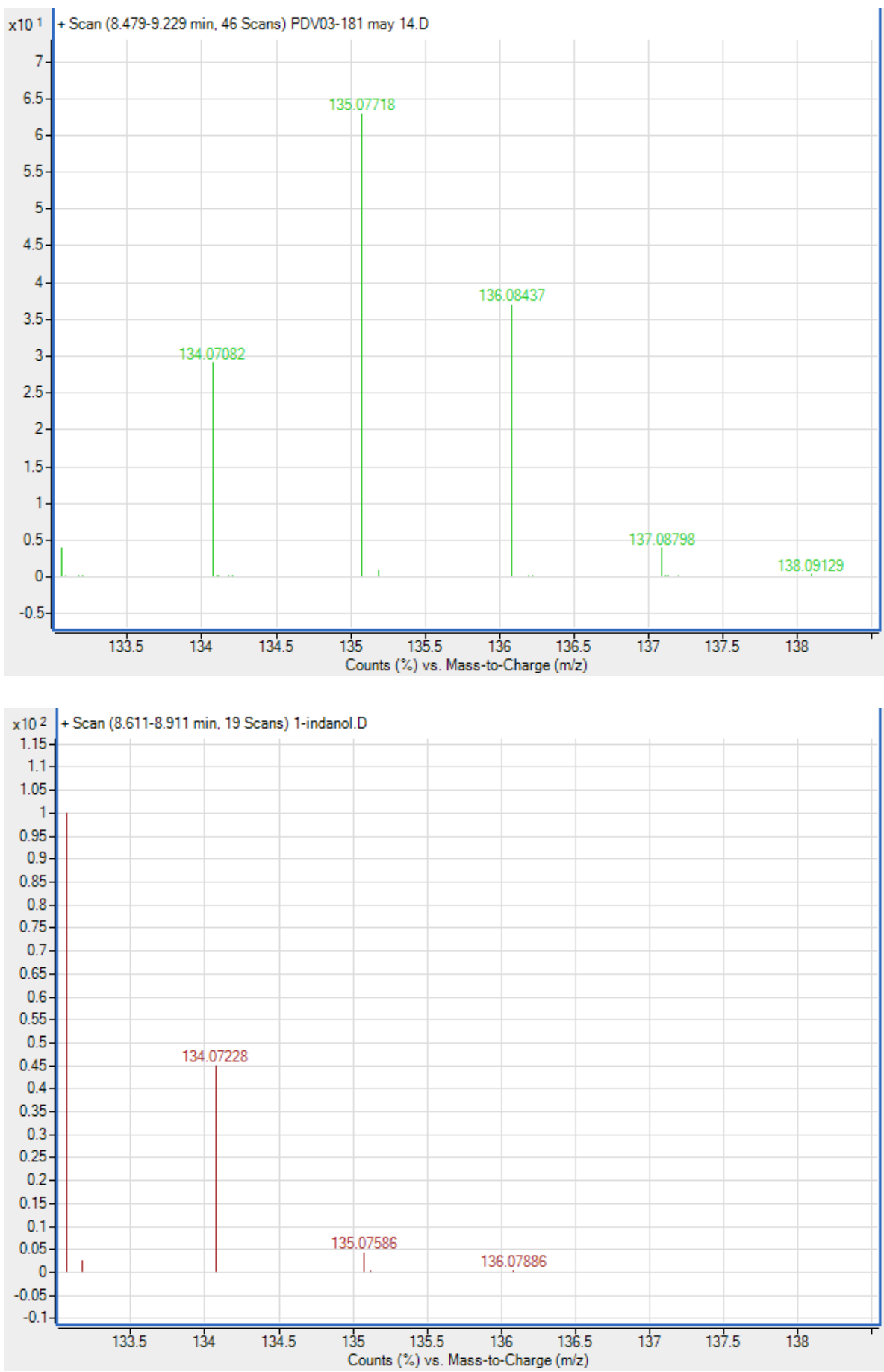

Figure S30. Deuterated indanol 7f-d (top). Natural Abundance (bottom). 


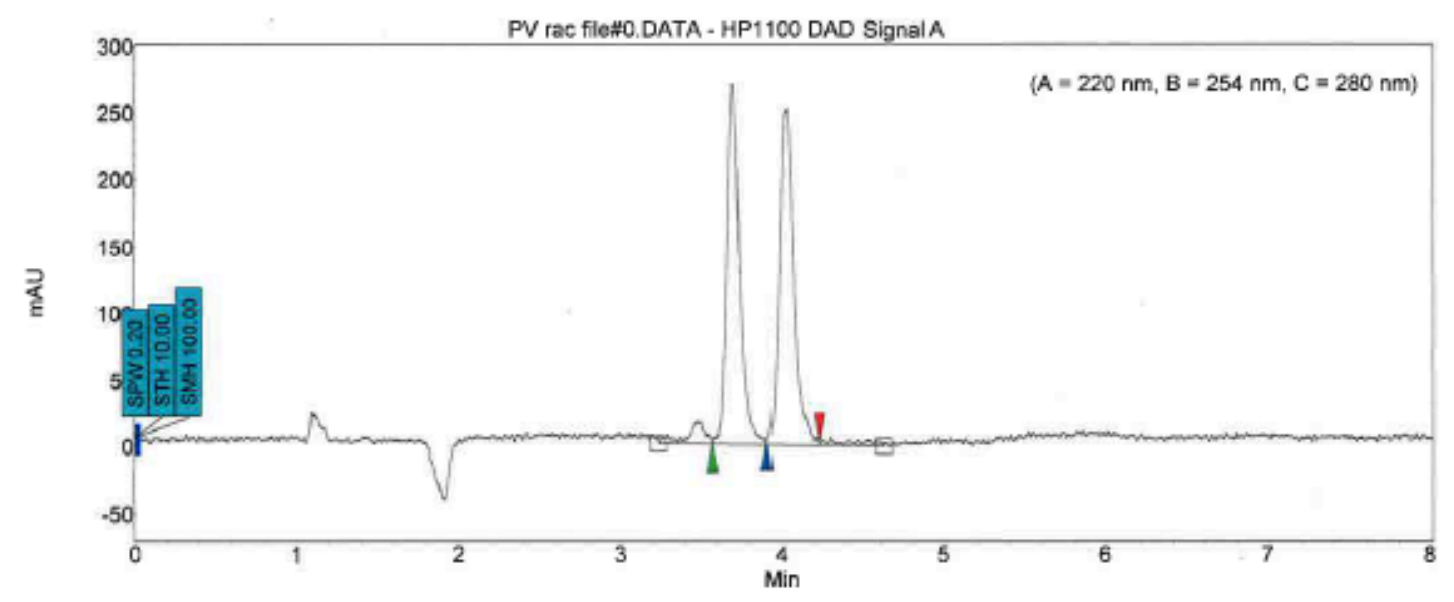

\begin{tabular}{|c|r|r|r|r|r|r|r|}
\hline Index & Time & Width & Height & Res. HW & Selectivity & Area & Area \\
\hline \hline & [Min] & {$[$ Min] } & {$[\mu \mathrm{M}]$} & & & {$[\mu$ V.Min] } & {$[\%]$} \\
\hline 1 & 3.69 & 0.08 & 269.5 & 0.00 & 0.00 & 24.9 & 49.905 \\
\hline 2 & 4.02 & 0.09 & 251.6 & 2.28 & 1.09 & 25.1 & 50.195 \\
\hline & & & & & & & \\
\hline Total & & & 521.1 & & & 50.0 & 100.000 \\
\hline
\end{tabular}

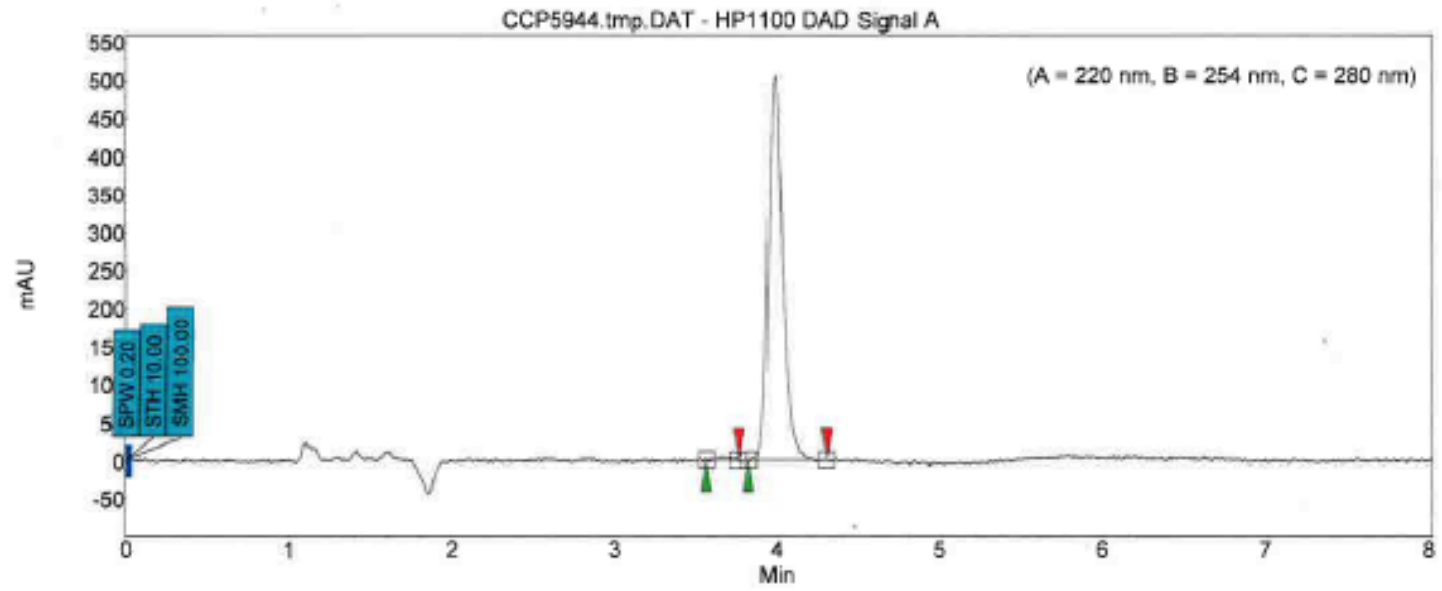

\begin{tabular}{|c|r|r|r|r|r|r|r|}
\hline Index & Time & Width & Height & Res. HW & Selectivity & Area & Area \\
\hline & {$[$ Min] } & {$[$ Min] } & [jV] & & & [UV.Min] & {$[\%]$} \\
\hline 2 & 3.66 & 0.01 & 4.5 & 0.00 & 0.00 & 0.1 & 0.264 \\
\hline 1 & 3.98 & 0.09 & 503.9 & 3.93 & 1.09 & 48.6 & 99.736 \\
\hline & & & & & & & \\
\hline Total & & & 508.4 & & & 48.8 & 100.000 \\
\hline
\end{tabular}

Figure S31. Chiral SFC Chromatogram of racemic (top) and enantioenriched (bottom) 7f-d. 

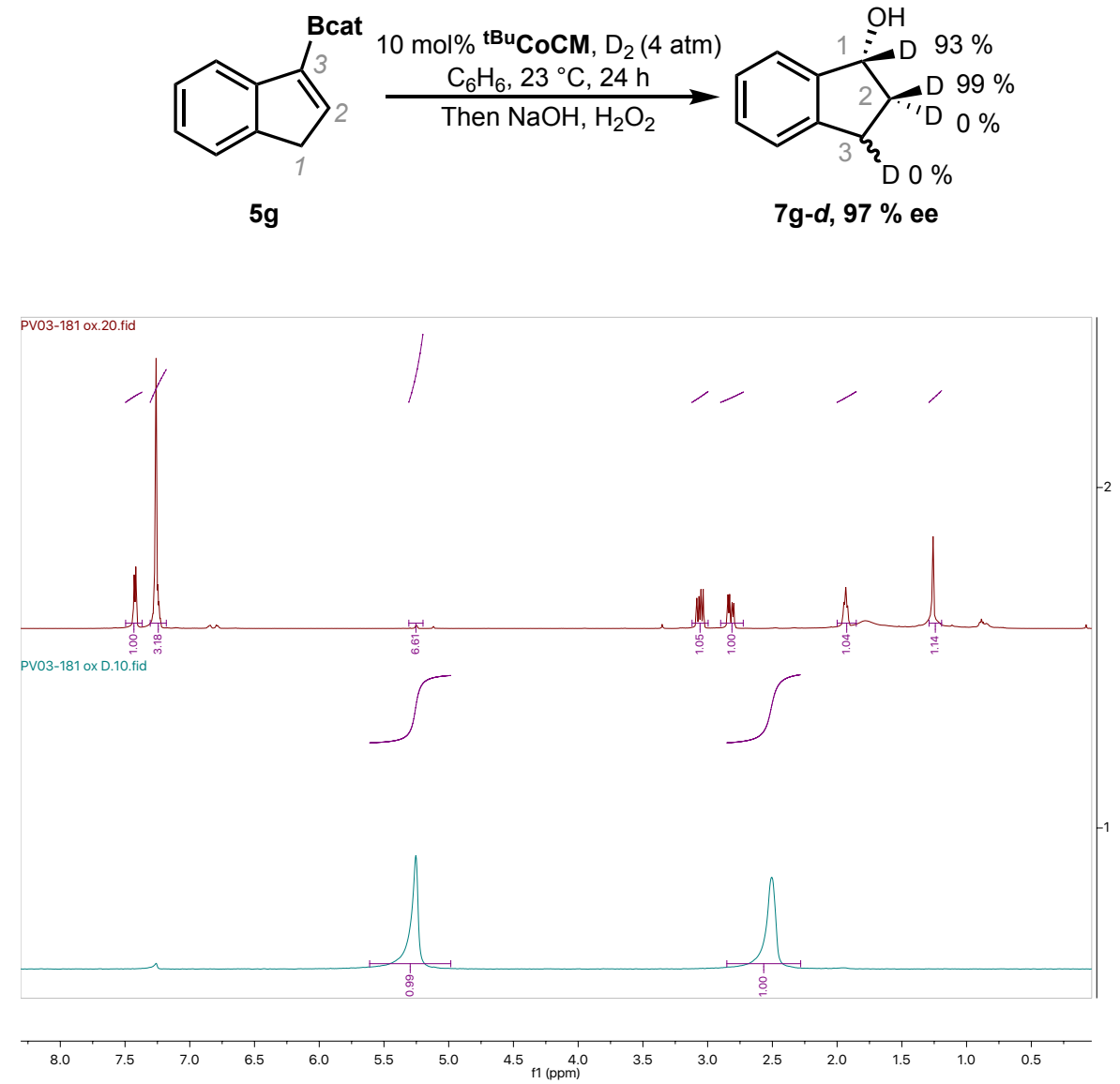

Figure S32. ${ }^{1} \mathrm{H}$ NMR of deuterated 1-indanol (top). ${ }^{2} \mathrm{H}$ NMR of deuterated 1-indanol (bottom).

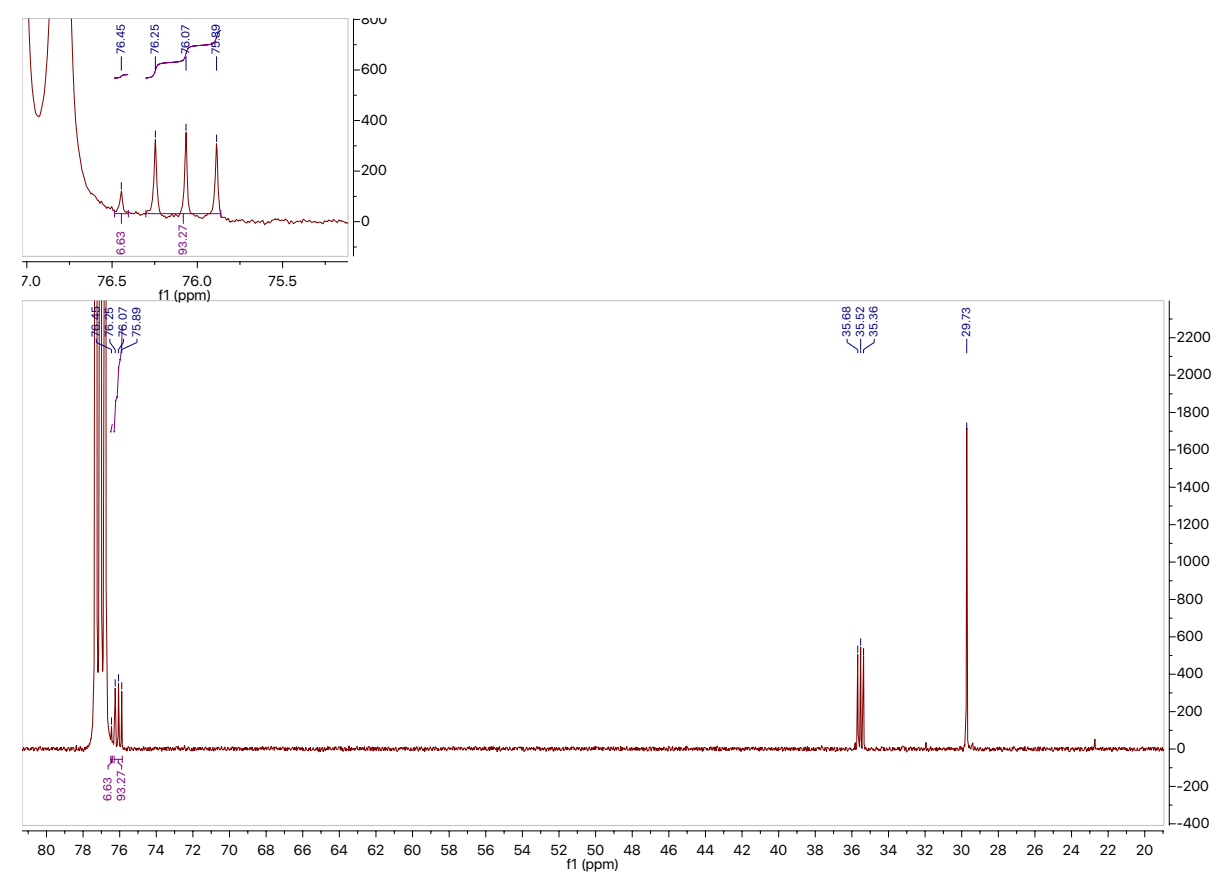

Figure S33. Quant. ${ }^{13} \mathrm{C}$ of deuterated 1-indanol. $7 \%$ un-deuterated C-1 observed. 


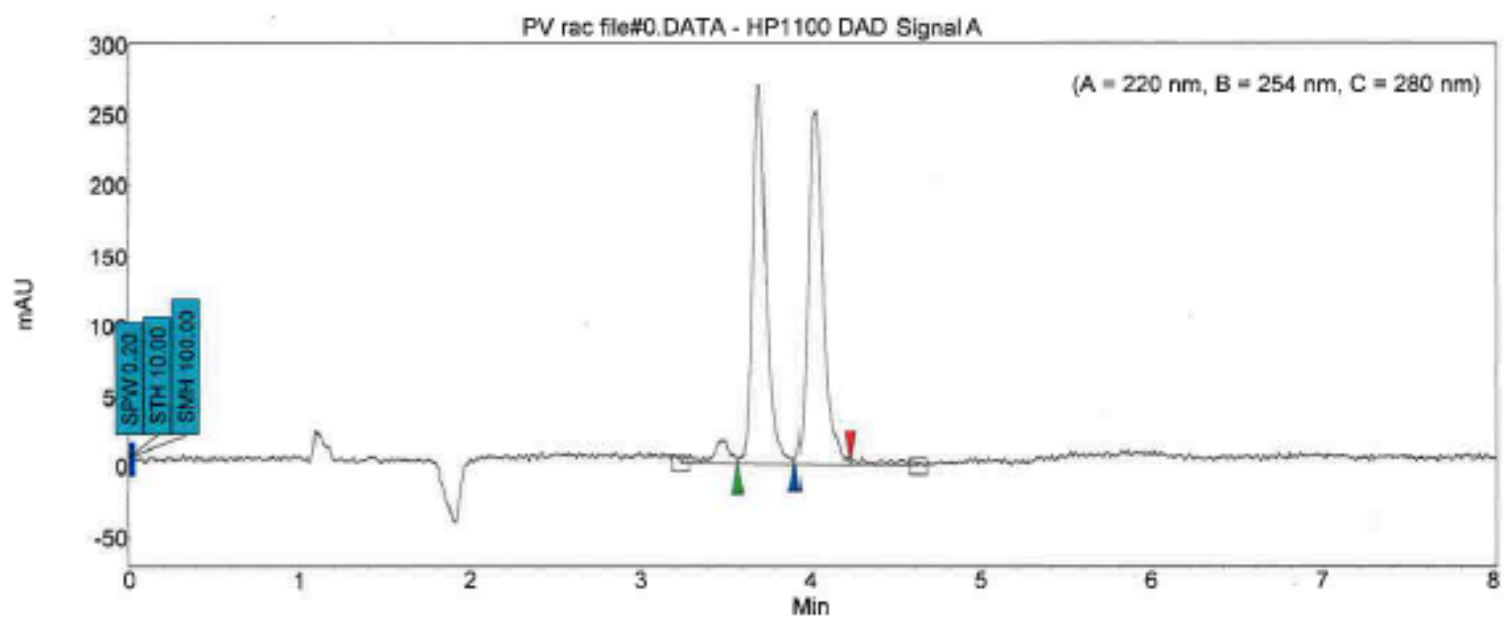

\begin{tabular}{|c|r|r|r|r|r|r|r|}
\hline Index & Time & Width & Height & Res. HW & Selectivity & Area & Area \\
\hline \hline & {$[$ Min] } & {$[$ Min] } & {$[\mu \mathrm{M}]$} & & & {$[\mu \mathrm{V}$. Min] } & {$[\%]$} \\
\hline 1 & 3.69 & 0.08 & 269.5 & 0.00 & 0.00 & 24.9 & 49.805 \\
\hline 2 & 4.02 & 0.09 & 251.6 & 2.28 & 1.09 & 25.1 & 50.195 \\
\hline & & & & & & & \\
\hline Total & & & 521.1 & & & 50.0 & 100.000 \\
\hline
\end{tabular}

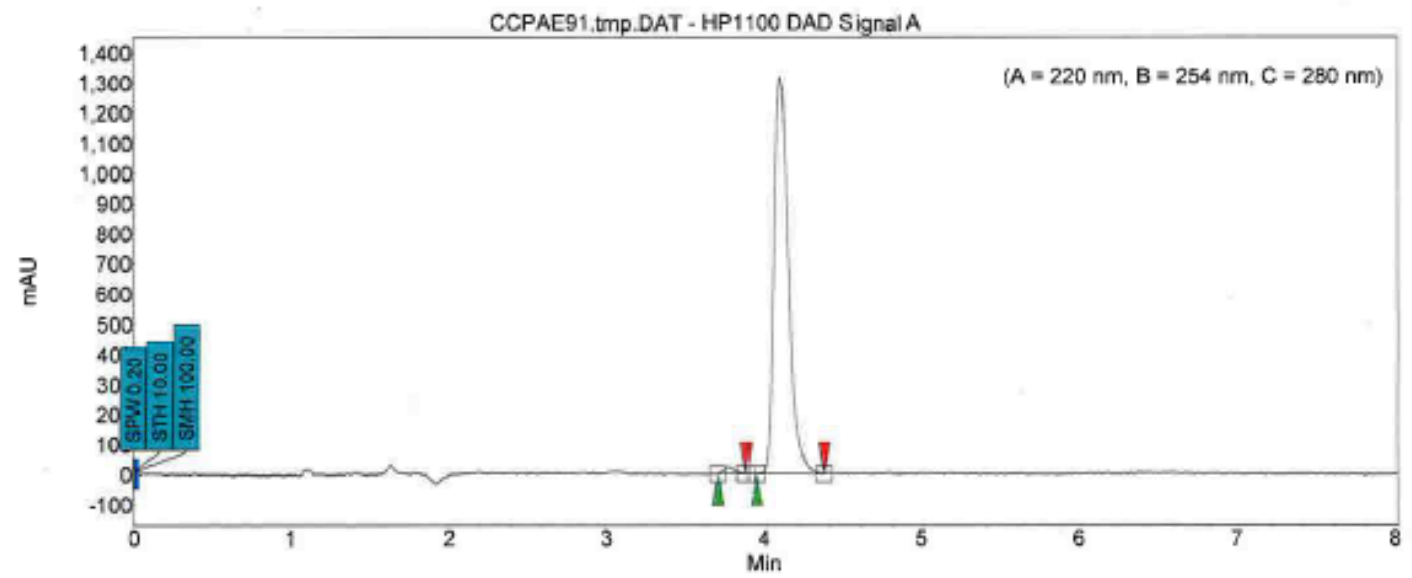

\begin{tabular}{|c|r|r|r|r|r|r|r|}
\hline Index & Time & Width & Height & Res. HW & Selectivity & Area & Area \\
\hline \hline & [Min] & {$[$ Min] } & {$[\mu \mathrm{V}]$} & & & {$[\mu \mathrm{V}$. Min] } & {$[\%]$} \\
\hline 1 & 3.78 & 0.07 & 22.5 & 0.00 & 0.00 & 1.8 & 1.251 \\
\hline 2 & 4.09 & 0,10 & 1317.0 & 2.22 & 1.08 & 139.5 & 98.749 \\
\hline & & & & & & & \\
\hline Total & & & 1339.4 & & & 141.2 & 100.000 \\
\hline
\end{tabular}

Figure S34. Chiral SFC Chromatogram of racemic (top) and enantioenriched (bottom) $\mathbf{7 g}-\boldsymbol{d}$. 
HRMS (El+):
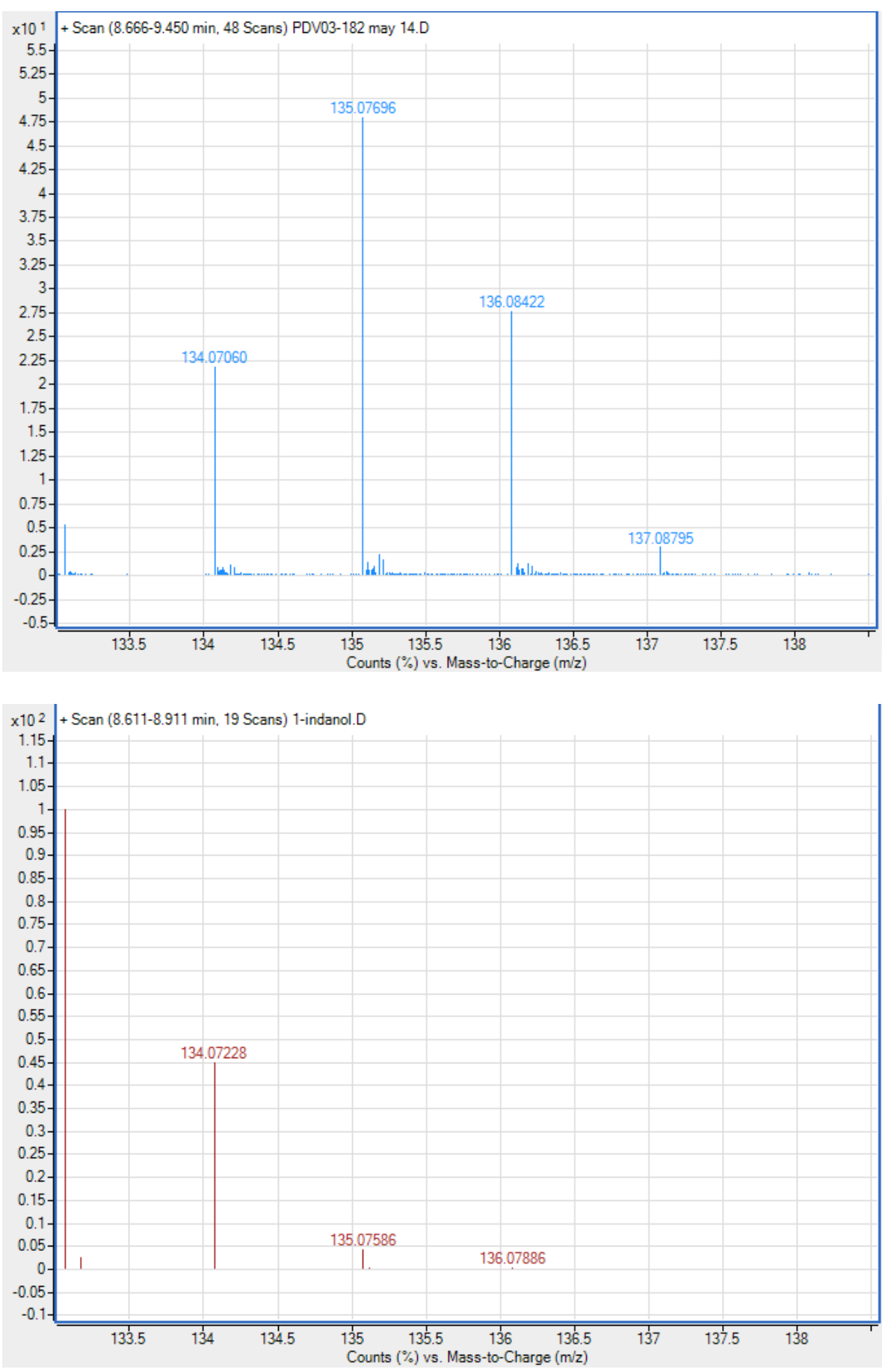

Figure S35. Deuterated indanol 7f- $\boldsymbol{d}$ (top). Natural Abundance (bottom). 
Catalytic Deuteration of $1 \mathrm{i}$ : Using a modified version of general procedure $B$. $D_{2}$ gas was used instead of $\mathrm{H}_{2}$ gas.

${ }^{1} \mathrm{H}$ NMR $(500 \mathrm{MHz}$, Chloroform-d) $\delta 7.31(\mathrm{t}, J=7.6 \mathrm{~Hz}, 2 \mathrm{H}), 7.26-7.18(\mathrm{~m}, 3 \mathrm{H}), 7.11$ (dd, $J=$ 8.3, 7.3 Hz, 2H), 7.01 (dd, $J=8.4,1.0 \mathrm{~Hz}, 2 \mathrm{H}$ ), 6.29 (dd, J = 7.4, $1.0 \mathrm{~Hz}, 2 \mathrm{H}$ ), 5.80 (s, 2H), 2.74 (dd, $J=13.4,5.5 \mathrm{~Hz}, 1 \mathrm{H}$ ), 2.62 (dd, $J=13.4,9.8 \mathrm{~Hz}, 1 \mathrm{H}$ ), 1.97 (dd, $J=9.8,5.5 \mathrm{~Hz}, 1 \mathrm{H}$ ), 1.29 (s, $6 \mathrm{H}), 1.28(\mathrm{~s}, 6 \mathrm{H})$.

${ }^{2} \mathrm{H}$ NMR $(77 \mathrm{MHz},) \delta 1.76,0.76$.

${ }^{13} \mathrm{C} \mathrm{NMR}\left(126 \mathrm{MHz}, \mathrm{CDCl}_{3}\right) \delta 142.57,141.27,136.29,128.55,128.36,127.58,125.82,119.52$, 117.30, 105.49, 83.30, 38.35, $28.13(\mathrm{t}, \mathrm{J}=19.41 \mathrm{~Hz}), 25.14,24.54$.

HRMS (ESI+): $m / z$ calculated for $\mathrm{C}_{25} \mathrm{H}_{29} \mathrm{D}_{2} \mathrm{~B}_{2} \mathrm{~N}_{2} \mathrm{O}_{2}{ }^{+}\left([\mathrm{MH}]^{+}\right)$: 415.2692 ; found: 415.2688 .
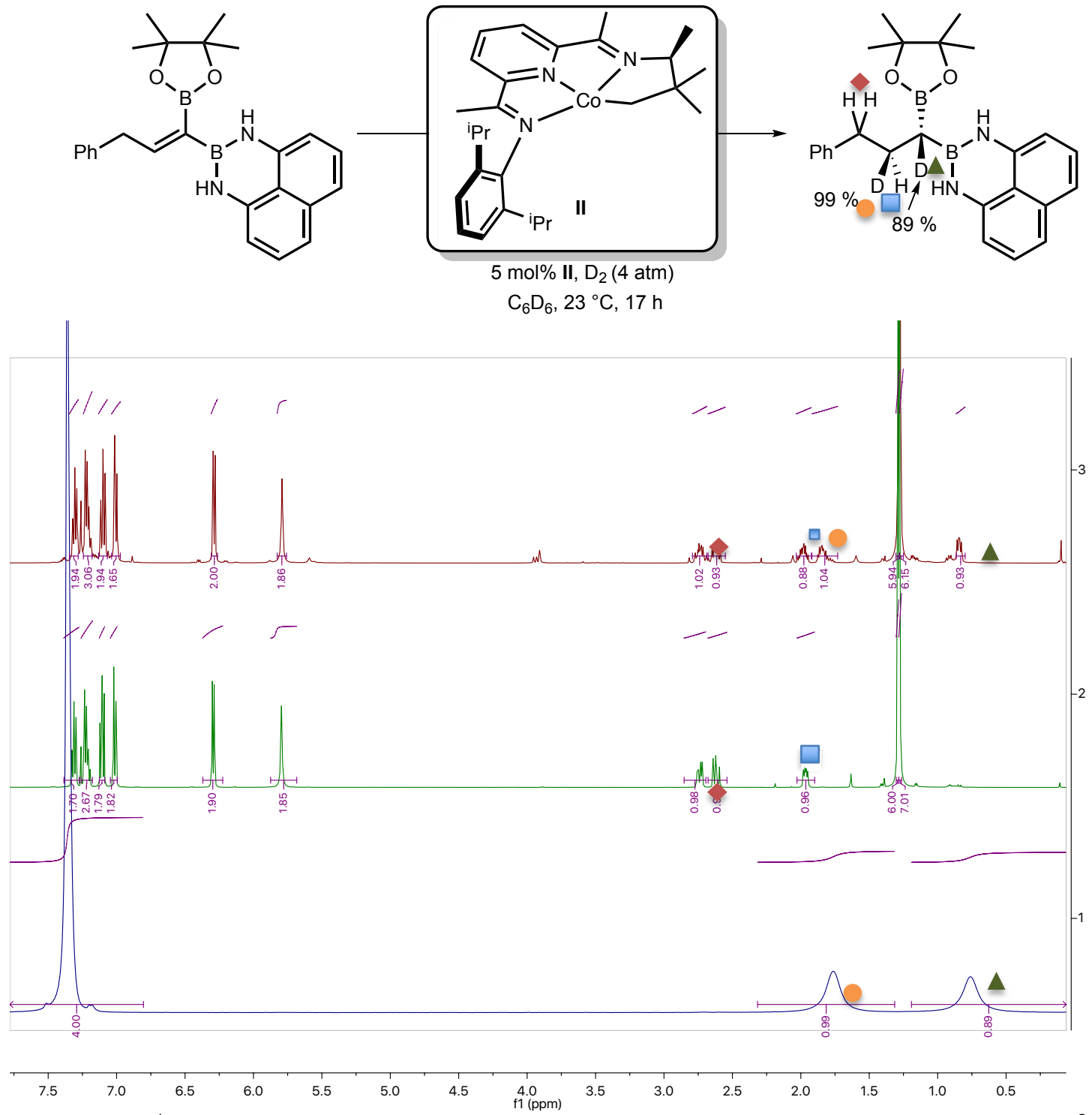

Figure S36. ${ }^{1} \mathrm{H}$ NMR Spectra of natural abundance (top), and deuterated (middle) $1 \mathrm{i} .{ }^{2} \mathrm{H}$ NM $\mathrm{R}$ spectra of deuterated $\mathbf{1 i}$. 


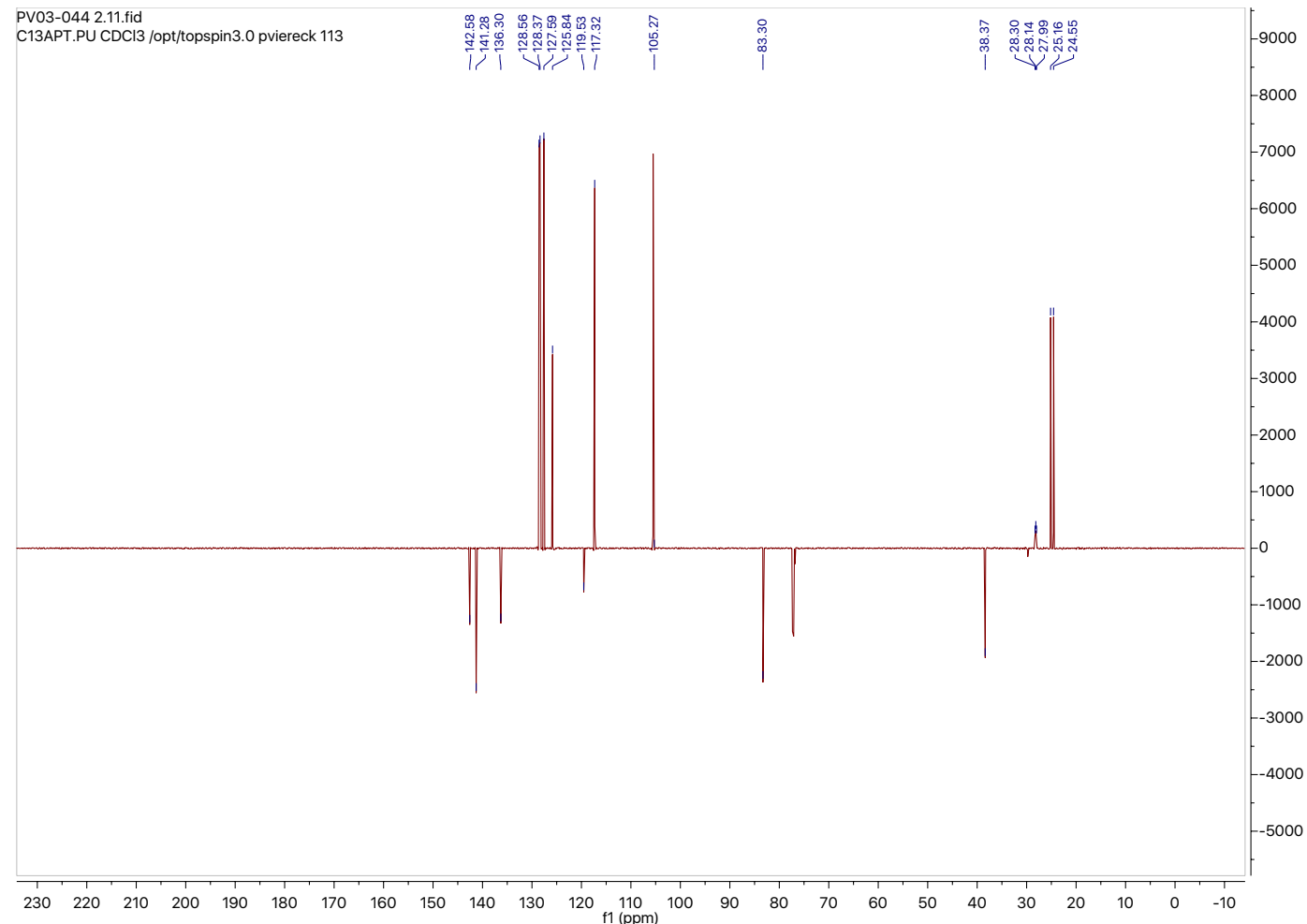

Figure S37. ${ }^{13} \mathrm{C}(\mathrm{APT})$ NMR Spectrum of deuterated 1i

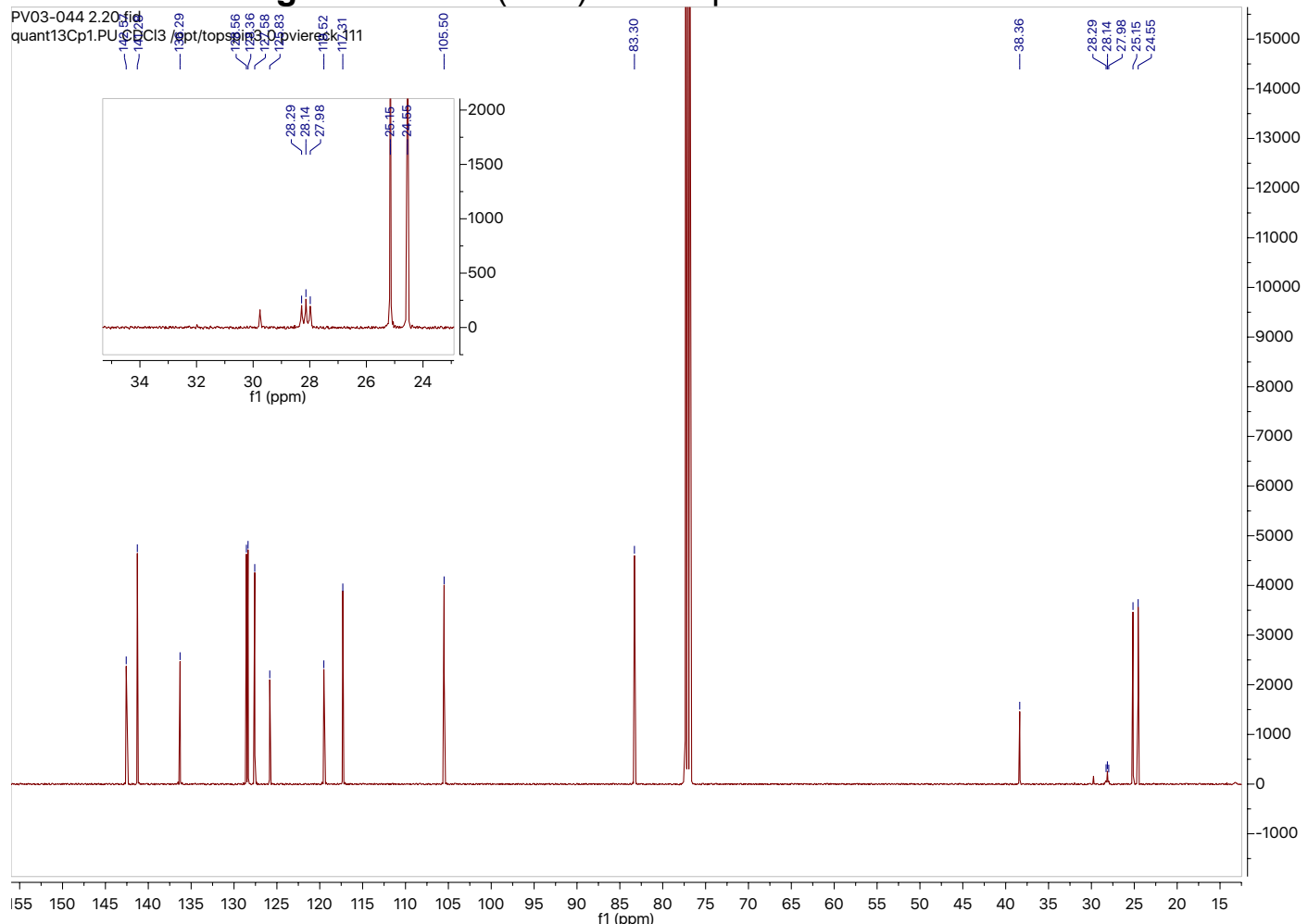

Figure S38. Quantitative ${ }^{13} \mathrm{C}$ NMR Spectrum of deuterated 1i 
Stoichiometric Deuteration of $1 \mathrm{i}:$ In a $20 \mathrm{~mL}$ vial, ${ }^{\mathrm{tBu}} \mathrm{CoCM}(26 \mathrm{mg}, 0.055 \mathrm{mmol}, 1.1$ equiv.) and dehydro-1i (21 mg, $0.05 \mathrm{mmol}, 1$ equiv.) was dissolved in $\mathrm{C}_{6} \mathrm{D}_{6}(0.3 \mathrm{~mL})$. The solution was transferred to a J-Young tube with a $1 \mathrm{~mL}$ syringe and rinsed once with $\mathrm{C}_{6} \mathrm{D}_{6}(0.1 \mathrm{~mL})$. The JYoung tube was attached to a high vacuum line, the solution frozen. The tube evacuated and backfilled with $D_{2}(1 \mathrm{~atm}$.) at room temperature. The tube was rotated for $17 \mathrm{~h}$, and full conversion of alkene was observed by ${ }^{1} \mathrm{H}$ NMR. The solution was passed through a silica plug, and the solvent removed. To remove free ligand, the product was purified with silica gel column chromatography hexanes:ethyl acetate (50:1). $19 \mathrm{mg}, 85 \%$ yield.
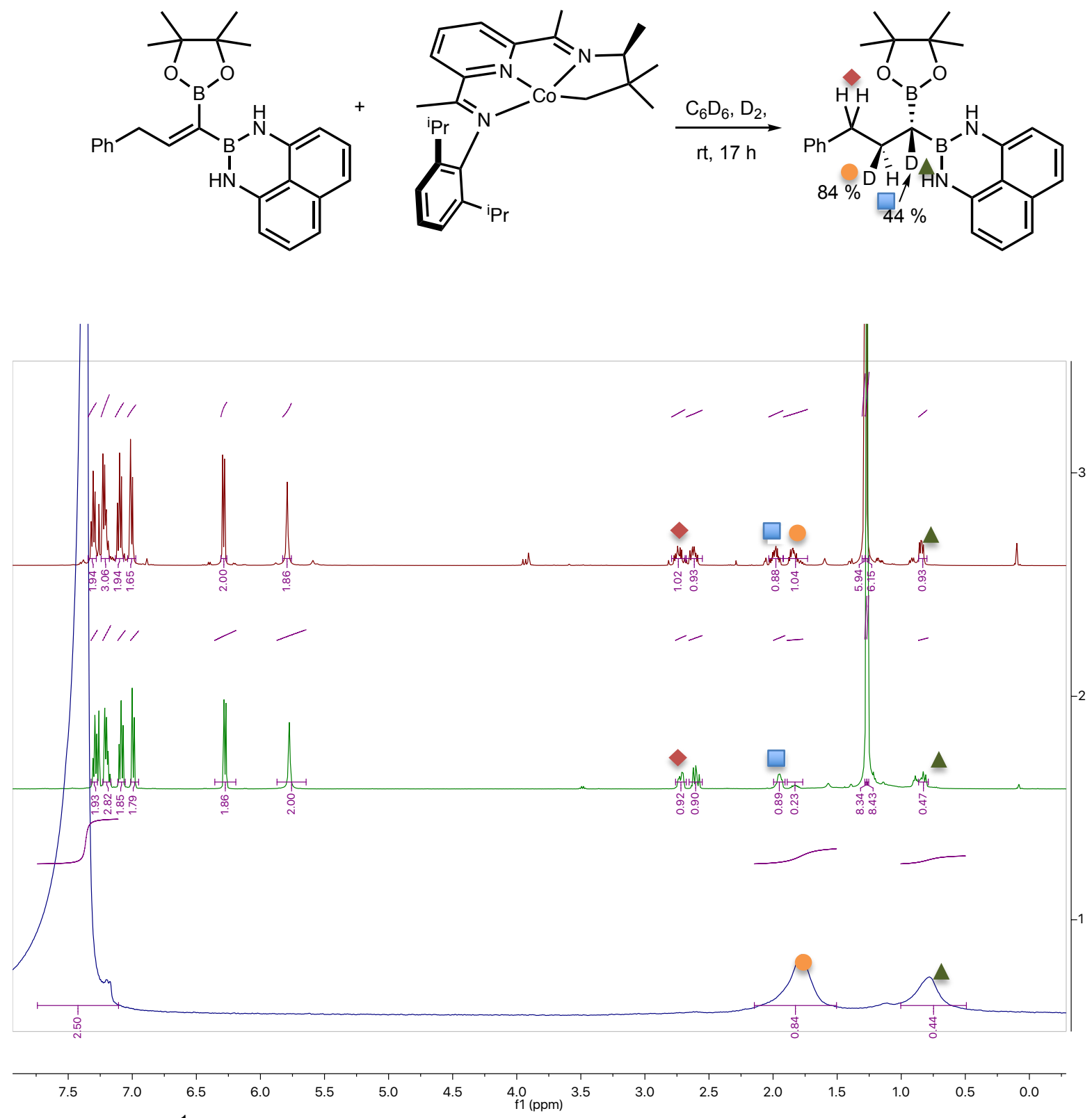

Figure S39. ${ }^{1} \mathrm{H}$ NMR Spectra of natural abundance (top), and partially deuterated (middle) $1 \mathrm{i}$. ${ }^{2} \mathrm{H}$ NMR spectra of partially deuterated $\mathbf{1 i}$. 


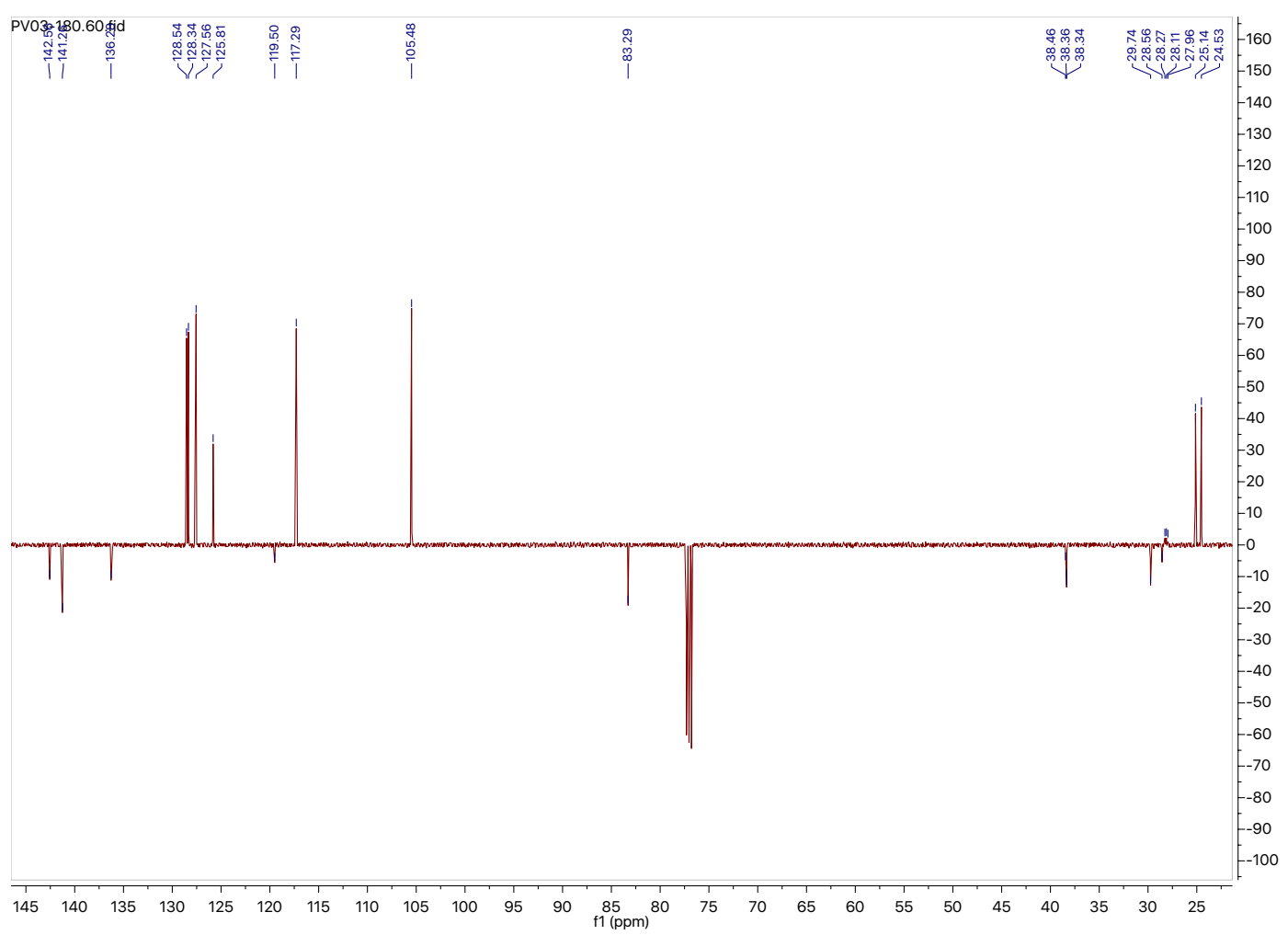

Figure S40. ${ }^{13} \mathrm{C}(\mathrm{NMR})$ NMR Spectrum of partially deuterated $1 \mathrm{i}$

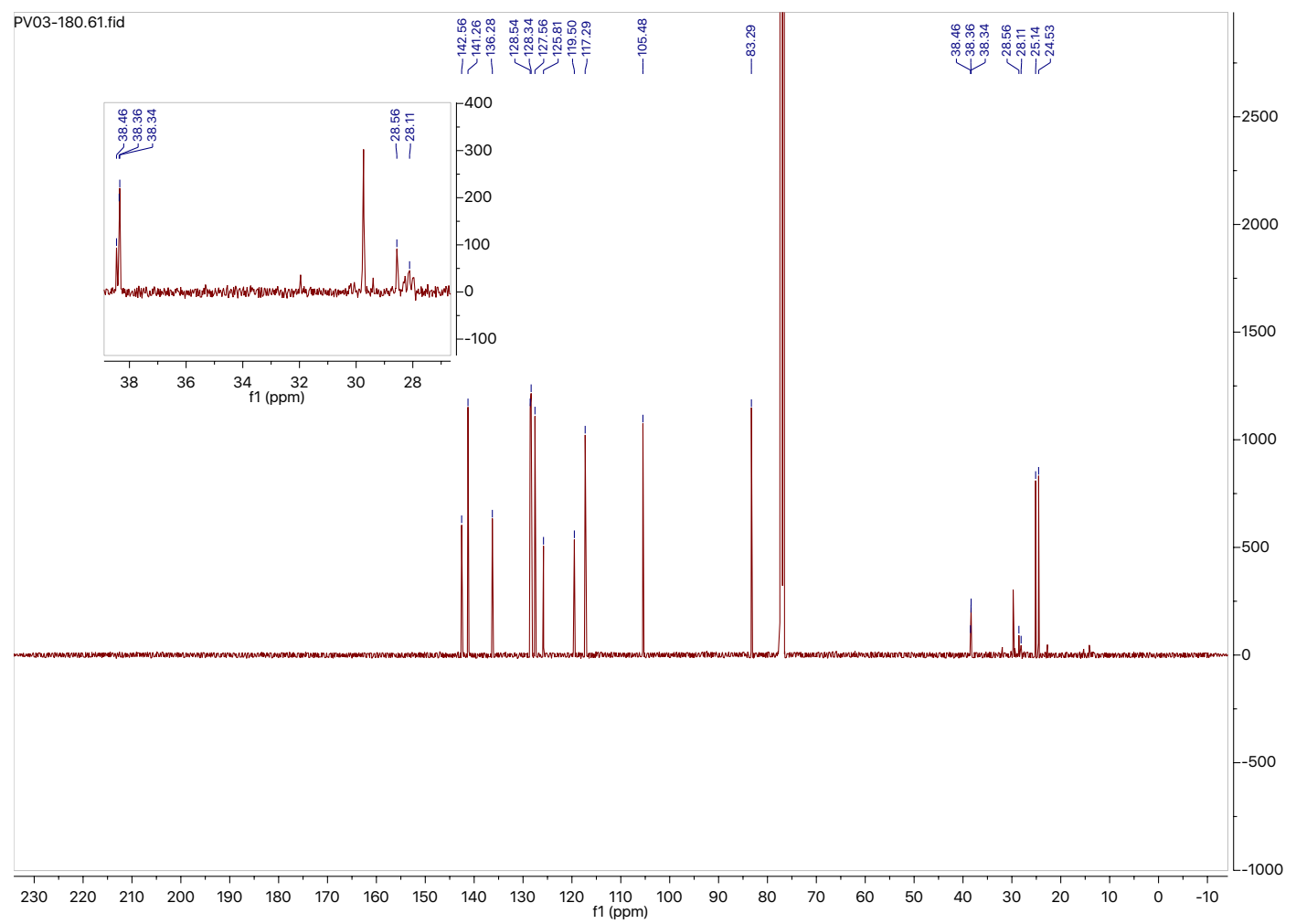

Figure S41. Quantitative ${ }^{13} \mathrm{C}$ NMR Spectrum of partially deuterated 1i 


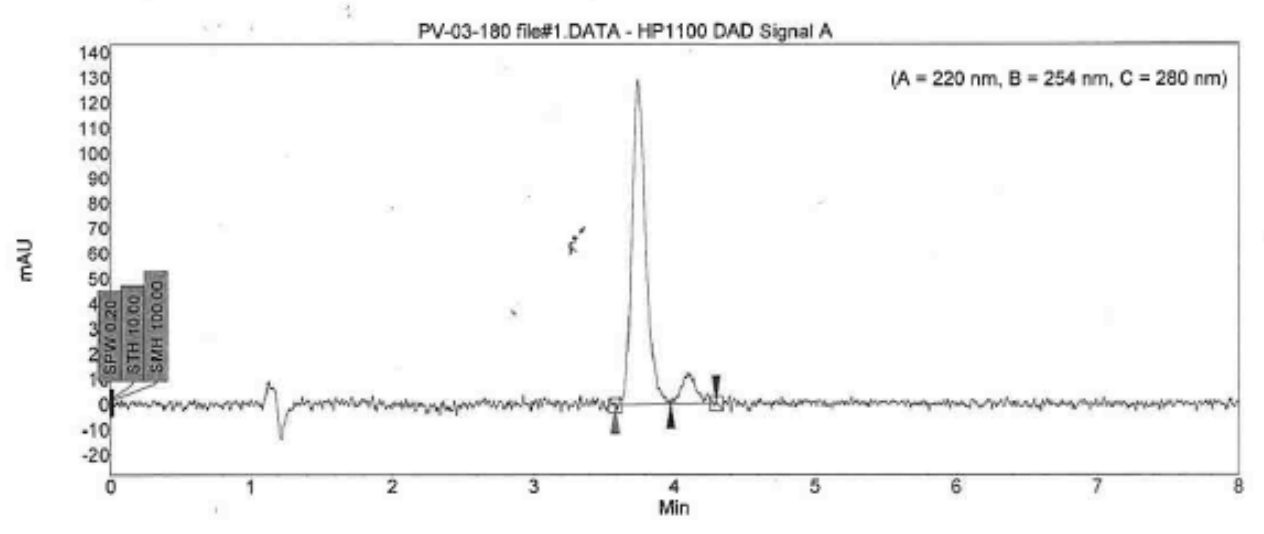

\begin{tabular}{|c|r|r|r|r|r|r|r|}
\hline Index & Time & Width & Height & Res. HW & Selectivily & Area & Area \\
\hline & {$[$ Min] } & {$[$ Min] } & {$[\mu \mathrm{V}]$} & & & {$[\mu \mathrm{V}$. Min] } & {$[\%]$} \\
\hline 1 & 3.74 & 0.11 & 129.4 & 0.00 & 0.00 & 14.9 & 90.734 \\
\hline 2 & 4.10 & 0.12 & 12.5 & 1.90 & 1.09 & 1.5 & 9.266 \\
\hline & & & & & & & \\
\hline Total & & & 141.9 & & & 16.4 & 100.000 \\
\hline
\end{tabular}

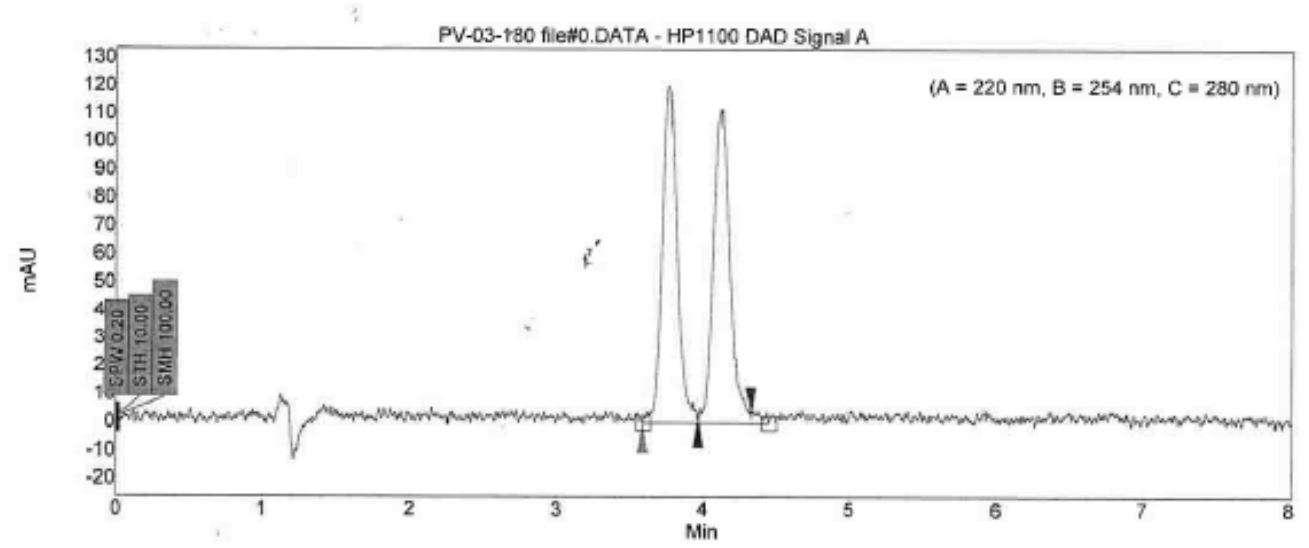

\begin{tabular}{|c|r|r|r|r|r|r|r|}
\hline Index & Time & Width & Height & Res. HW & Selectivity & Area & Area \\
\hline \hline & [Min] & {$[$ Min] } & {$[\mu$ ] } & & & {$[$ JVMin] } & {$[\%]$} \\
\hline 1 & 3.77 & 0.10 & 119.9 & 0.00 & 0.00 & 14.3 & 49.478 \\
\hline 2 & 4.13 & 0.12 & 111.6 & 1.92 & 1.10 & 14.6 & 50.522 \\
\hline & & & & & & & \\
\hline Total & & & 231.6 & & & 28.8 & 100.000 \\
\hline
\end{tabular}

Figure S42. SFC Chromatogram of $\mathbf{2} \mathbf{i}$ from stoichiometric deuteration experiment (top). Racemic 2i (bottom). 

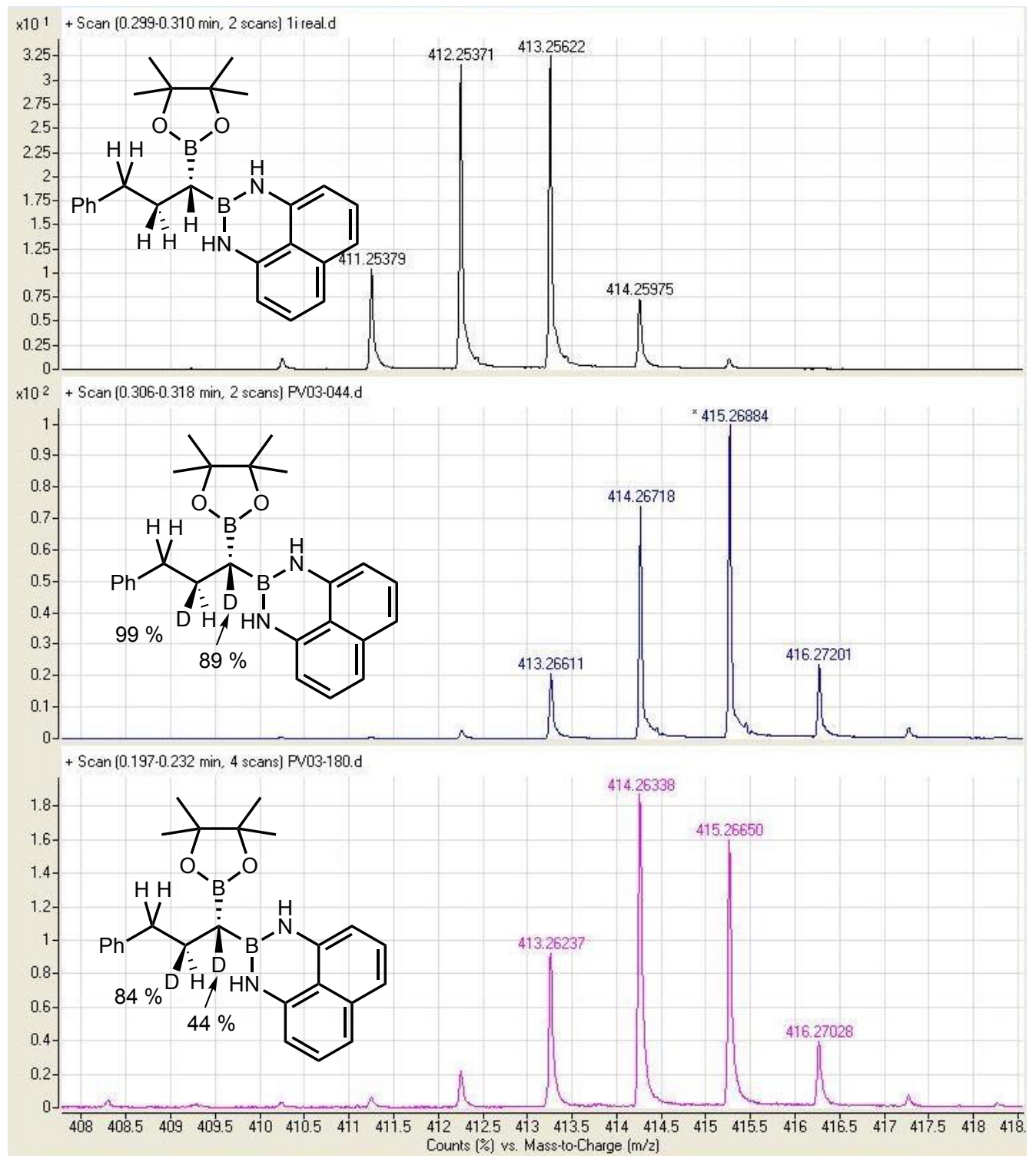

Figure S43. LC-HRMS (ESI+) of Deuterated Diboryl Alkenes. The propensity of the compound to form a cation radical prohibits comparison of the natural abundance isotopic distribution to the deuterated analogue. It is clear however that the catalytic experiments results primarily in the dideuterated product and the stoichiometric experiment results primarily in mono-deuterated product. 
E/Z Isomerization of $1 \mathrm{i}$ : In a $20 \mathrm{~mL}$ vial, ( $\left.{ }^{\mathrm{Me} P D I}\right) \mathrm{Fe}\left(\mathbf{N}_{2}\right)(3 \mathrm{mg}, 0.0085 \mathrm{mmol}, 5 \mathrm{~mol} \%)$ and $1 \mathrm{i}$ (70 mg, $0.171 \mathrm{mmol}, 1$ equiv.) was dissolved in $\mathrm{C}_{6} \mathrm{D}_{6}(0.4 \mathrm{~mL})$. The solution was transferred to a J-Young tube with a $1 \mathrm{~mL}$ syringe and rinsed once with $\mathrm{C}_{6} \mathrm{D}_{6}(0.1 \mathrm{~mL})$. The J-Young tube was attached to a high vacuum line, the solution frozen. The tube evacuated and backfilled with $\mathrm{H}_{2}$ ( $0.1 \mathrm{~atm}$.) at room temperature. The tube was rotated for $2 \mathrm{~h}$, and $\mathrm{E} / \mathrm{Z}$ isomerization observed by ${ }^{1} \mathrm{H}$ NMR. The $\mathrm{J}$-Young tube was attached to a high vacuum line and the headspace evacuated. The contents of the tube were filtered through silica eluting with $\mathrm{C}_{6} \mathrm{H}_{6}$, and the solvent removed, affording an off-white solid (42 mg, $60 \%$ yield, $20 \%$ isomerization).<smiles>CC1(C)OB(C(=CCc2ccccc2)B2Nc3cccc4cccc(c34)N2)OC1(C)C</smiles>

${ }^{1} \mathrm{H}$ NMR $400 \mathrm{MHz}$, Benzene- $d_{6}$ ) [Z-Alkene (Major)] $\delta 7.27(\mathrm{~d}, J=7.5 \mathrm{~Hz}, 2 \mathrm{H}), 7.20$ (t, $J=7.5 \mathrm{~Hz}$, $2 \mathrm{H}), 7.08-6.96(\mathrm{~m}, 4 \mathrm{H}), 6.76(\mathrm{t}, \mathrm{J}=7.2 \mathrm{~Hz}, 1 \mathrm{H}), 6.19(\mathrm{~s}, 2 \mathrm{H}), 6.10(\mathrm{t}, J=4.2 \mathrm{~Hz}, 2 \mathrm{H}), 3.95(\mathrm{~d}$, $J=7.2 \mathrm{~Hz}, 2 \mathrm{H}), 1.04(\mathrm{~s}, 12 \mathrm{H})$.

${ }^{1} \mathrm{H}$ NMR (400 MHz, Benzene- $d_{6}$ ) [E-Alkene (Major)] $\delta 7.46(\mathrm{t}, J=7.3 \mathrm{~Hz}, 1 \mathrm{H}), 7.09$ (s, 4H), 7.06 $-6.98(\mathrm{~m}, 5 \mathrm{H}), 6.00(\mathrm{dd}, J=6.7,1.6 \mathrm{~Hz}, 2 \mathrm{H}), 5.74(\mathrm{~s}, 2 \mathrm{H}), 3.47$ (d, J = 7.3 Hz, 2H), 1.07 (s, $12 \mathrm{H})$.

PV05-026 1 scan.10.fid

PROTON.PU CDCl3 /opt/topspin3.0 pviereck 50

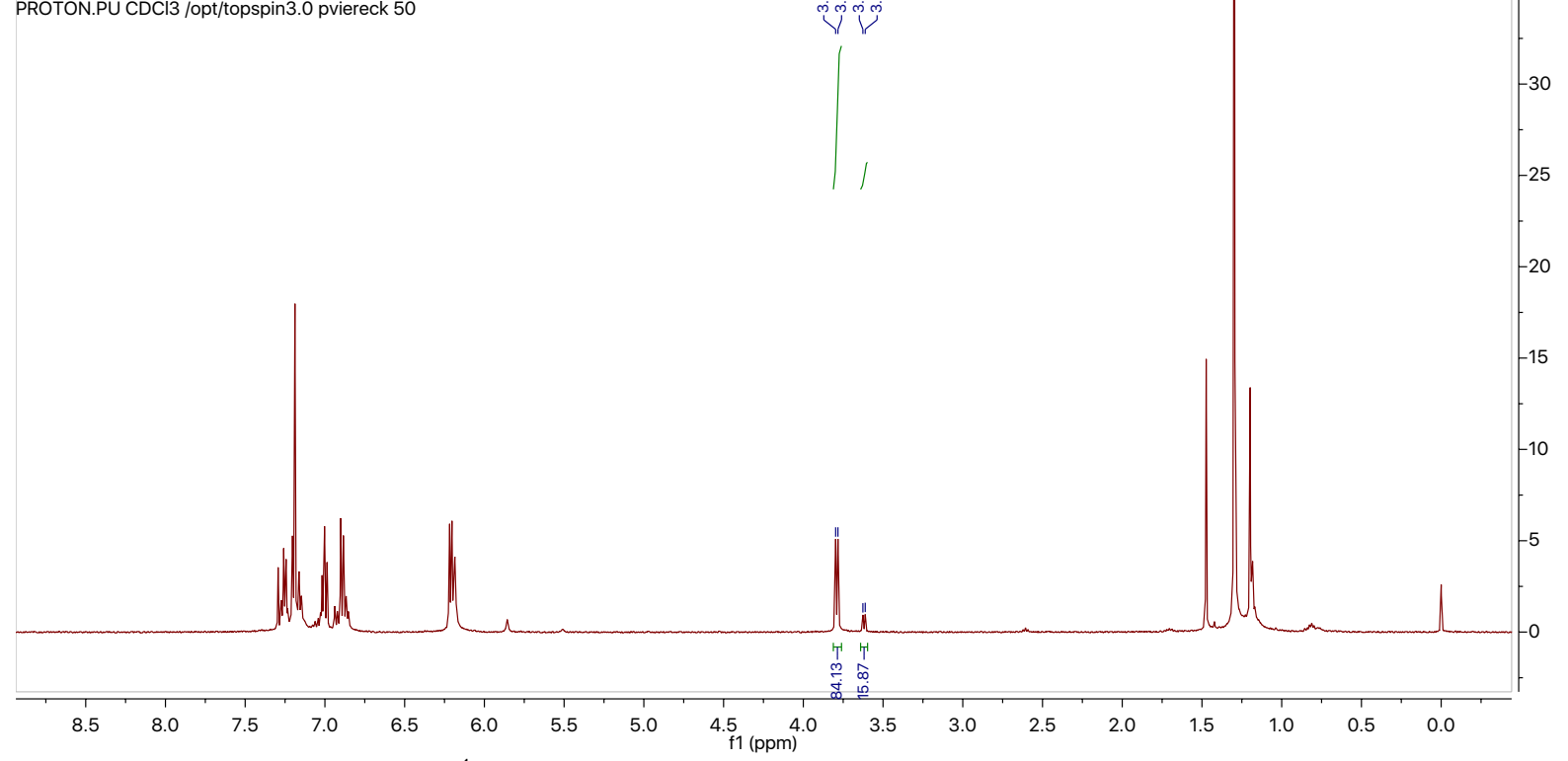

Figure S44. ${ }^{1} \mathrm{H}$ NMR (One Scan) Spectrum of the Isomerization Product. 
Deuteration of E/Z Mixture: To a $20 \mathrm{~mL}$ vial containing cobalt complex ${ }^{\mathrm{tBu}} \mathrm{CoCM}(5 \mathrm{mg}, 0.01$ $\mathrm{mmol}$ ) was added $\mathrm{E} / \mathrm{Z}$ mixture of $1 \mathrm{i}(42 \mathrm{mg}, 0.102 \mathrm{mmol})$ and benzene $(1.5 \mathrm{~mL})$. The solution was taken up in a syringe, and $0.75 \mathrm{~mL}$ of the solution was added to two separate oven dried, thick-walled glass vessels. Magnetic stir bars were added, and the bombs were sealed with Kontes valves. The vessels were brought out of the glovebox and connected to a high vacuum line. The vessels were frozen in liquid nitrogen, evacuated, and backfilled with $1 \mathrm{~atm}$ of hydrogen gas at liquid nitrogen temperature, corresponding to approximately 4 atm of hydrogen pressure at $23^{\circ} \mathrm{C}$. The frozen reaction solutions were then left to thaw, after which it was magnetically stirred for the period of time indicated. 15 minutes after thawing, one bomb was opened to air and filtered through silica gel, eluting with hexanes/ethyl acetate 10:1. This was done after 17 hours for the other bomb. The color of the reaction solution under an atmosphere of hydrogen was purple (the same shade of purple as when cobalt complex ${ }^{\mathrm{tBu}} \mathrm{CoCM}$ is dissolved in benzene). ${ }^{1} \mathrm{H}$ NMR taken in $\mathrm{CDCl}_{3}$. A new chiral separation method was developed for the analysis of the partially converted alkane, however poor baseline separation decreases the precision of the enantioselectivity.

HPLC (Chiralcel OD-H): $40 \% \mathrm{MeOH}(0.1 \% \mathrm{DEA}) / \mathrm{CO}_{2} 100 \mathrm{bar}, 3 \mathrm{~mL} / \mathrm{min}, \mathrm{T}_{\text {major }}=4.95 \mathrm{~min}$, $\mathrm{T}_{\text {minor }}=5.76 \mathrm{~min}, \mathrm{~T}_{\mathrm{E}-\mathrm{Alkene}}=5.28 \mathrm{~T}_{\text {Z-Alkene }}=7.72 \mathrm{~min}$.
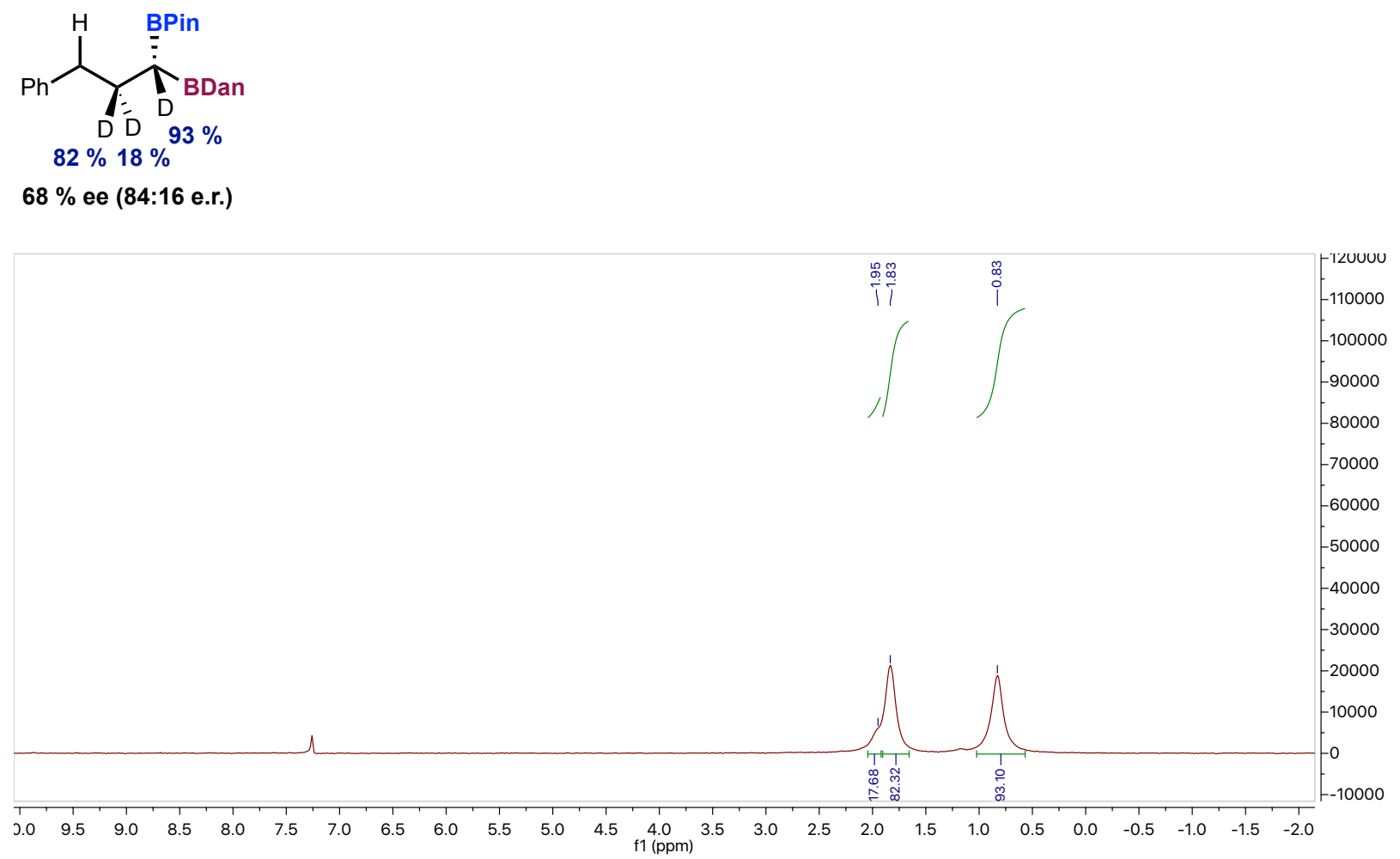

Figure S45. ${ }^{2} \mathrm{H}$ NMR of the Deuteration of $E / Z$ mixture. 


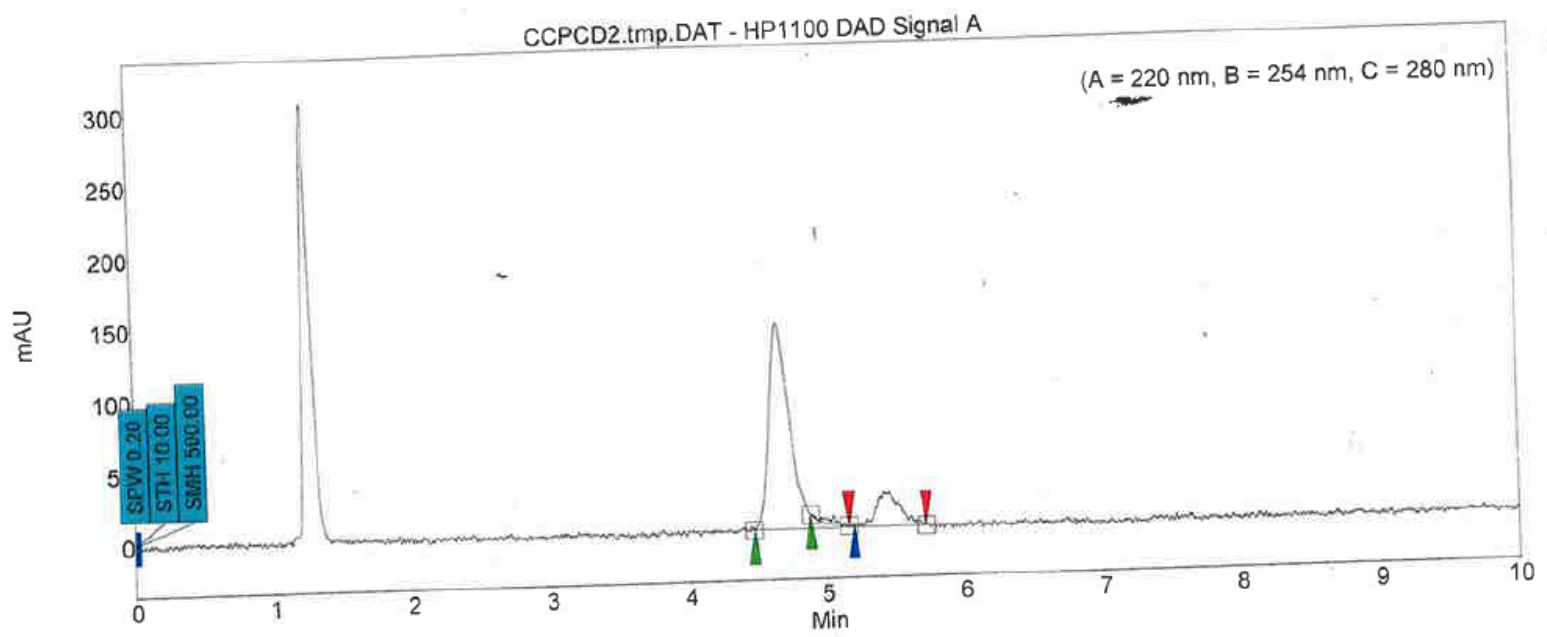

\begin{tabular}{|c|r|r|r|r|r|r|r|}
\hline Index & Time & Width & Height & Res. HW & Selectivity & Area & Area \\
\hline \hline & {$[$ Min] } & {$[$ Min] } & {$[\mu \mathrm{V}]$} & & & {$[\mu \mathrm{V}$. Min] $]$} & {$[\%]$} \\
\hline 1 & 4.66 & 0.15 & 144.0 & 0.00 & 0.00 & 25.1 & 82.876 \\
\hline 3 & 5.05 & 0.02 & 6.6 & 2.64 & 1.08 & 0.5 & 1.758 \\
\hline 2 & 5.42 & 0.18 & 23.6 & 2.14 & 1.07 & 4.7 & 15.365 \\
\hline & & & & & & & \\
\hline Total & & & 174.2 & & & 30.3 & 100.000 \\
\hline
\end{tabular}

Figure S46. SFC chromatogram of the deuterated product of the $E / Z$ mixture. 


\section{References}

${ }^{1}$ Friedfeld, M. R.; Shevlin, M.; Margulieux, G. W.; Campeau, L.-C.; Chirik, P. J. Cobalt-Catalyzed Enantioselective Hydrogenation of Minimally Functionalized Alkenes: Isotopic Labeling Provides Insight into the Origin of Stereoselectivity and Alkene Insertion Preferences. J. Am. Chem. Soc. 2016, 138 (10), 3314-3324.

2 Monfette, S.; Turner, Z. R.; Semproni, S. P.; Chirik, P. J. Enantiopure C1-Symmetric Bis(Imino)Pyridine Cobalt Complexes for Asymmetric Alkene Hydrogenation. J. Am. Chem. Soc. 2012, 134 (10), 4561-4564.

${ }^{3}$ Bruno, N. C.; Tudge, M. T.; Buchwald, S. L. Design and Preparation of New Palladium Precatalysts for C-C and C-N Cross-Coupling Reactions. Chem. Sci. 2013, 4 (3), 916-920.

${ }^{4}$ Pangborn, A. B.; Giardello, M. A.; Grubbs, R. H.; Rosen, R. K.; Timmers, F. J. Safe and Convenient Procedure for Solvent Purification. Organometallics 1996, 15, 1518-1520.

${ }^{5}$ Iwadate, N.; Suginome, M. Differentially Protected Diboron for Regioselective Diboration of Alkynes: Internal-Selective Cross-Coupling of 1-Alkene-1,2-Diboronic Acid Derivatives. J. Am. Chem. Soc. 2010, 132 (8), 2548-2549.

${ }^{6}$ lannazzo, L.; Vollhardt, K. P. C.; Malacria, M.; Aubert, C.; Gandon, V. Alkynylboronates and Boramides in Col- and Rhl-Catalyzed [2+2+2] Cycloadditions: Construction of Oligoaryls through Selective Suzuki Couplings. Eur. J. Org. Chem. 2011, 2011 (18), 3283-3292.

${ }^{7}$ Krautwald, S.; Bezdek, M. J.; Chirik, P. J. Cobalt-Catalyzed 1,1-Diboration of Terminal Alkynes: Scope, Mechanism, and Synthetic Applications. J. Am. Chem. Soc. 2017, 139 (10), 3868-3875.

8 Matthew, S. C.; Glasspoole, B. W.; Eisenberger, P.; Crudden, C. M. Synthesis of Enantiomerically Enriched Triarylmethanes by Enantiospecific Suzuki-Miyaura Cross-Coupling Reactions. J. Am. Chem. Soc., 2014, 136, 5828-5831.

9 Noh, D.; Yoon, S. K.; Won, J.; Lee, J. Y.; Yun, J. An Efficient Copper(I)-Catalyst System for the Asymmetric Hydroboration of $\beta$-Substituted Vinylarenes with Pinacolborane. Chemistry - An Asian Journal 2011, 6 (8), 1967-1969. 


\section{NMR Spectra and}

$$
\text { Chiral SFC }
$$

\section{Chromatograms of}

Isolated Compounds 


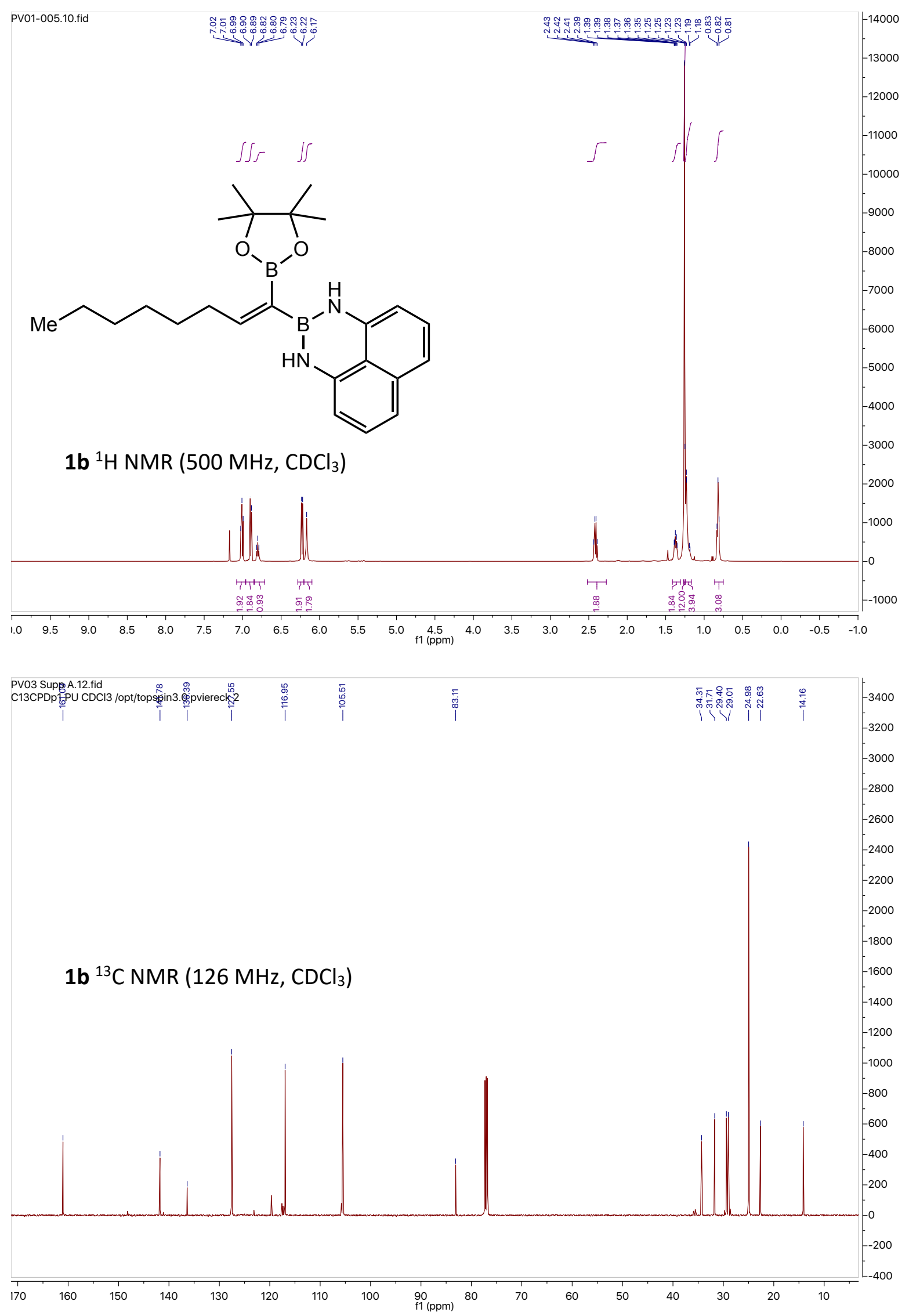



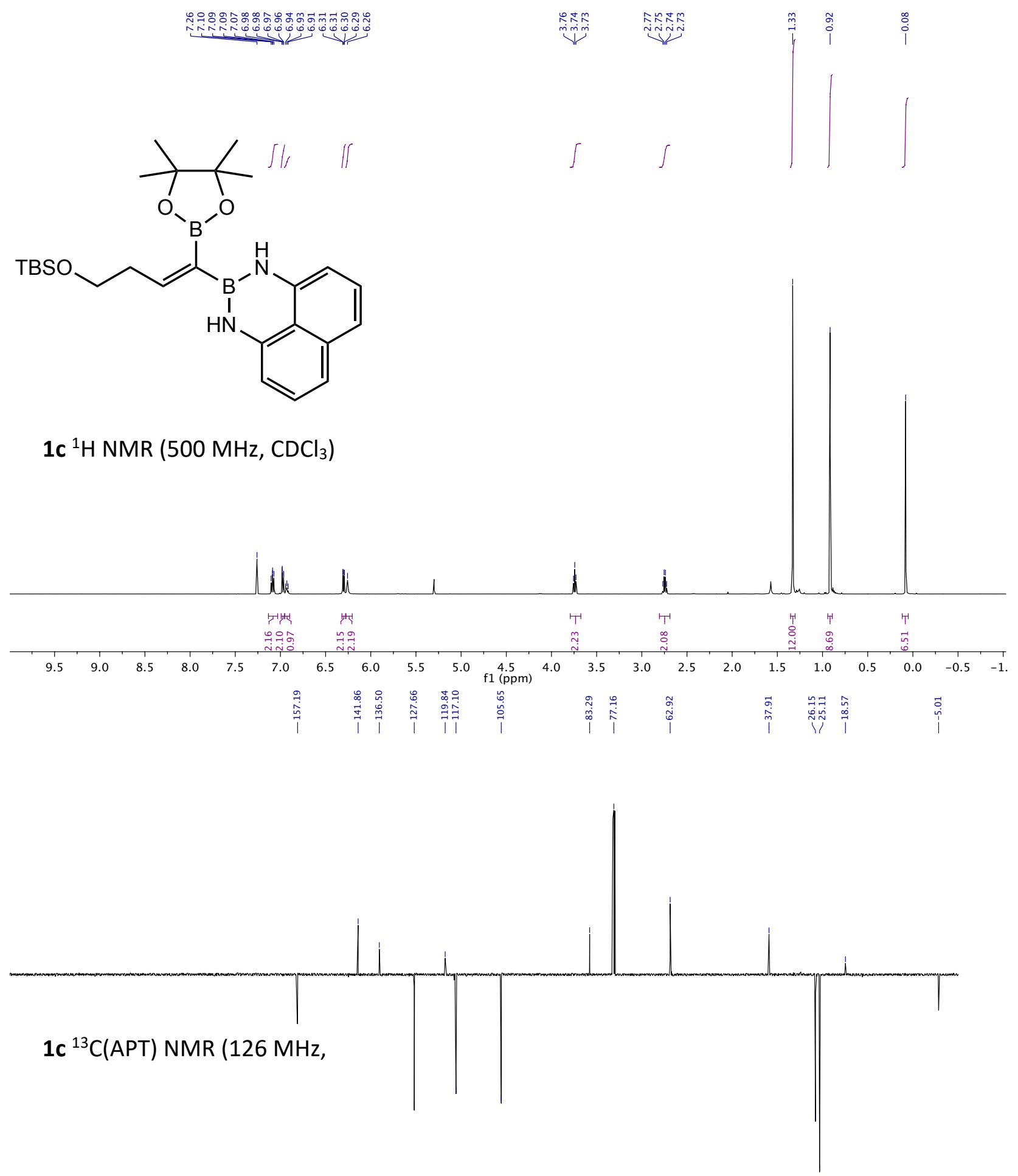

\begin{tabular}{llllllllllllllllllllllllllllll}
\hline 30 & 220 & 210 & 200 & 190 & 180 & 170 & 160 & 150 & 140 & 130 & 120 & 110 & 100 & 90 & 80 & 70 & 60 & 50 & 40 & 30 & 20 & 10 & 0 & -1
\end{tabular} 


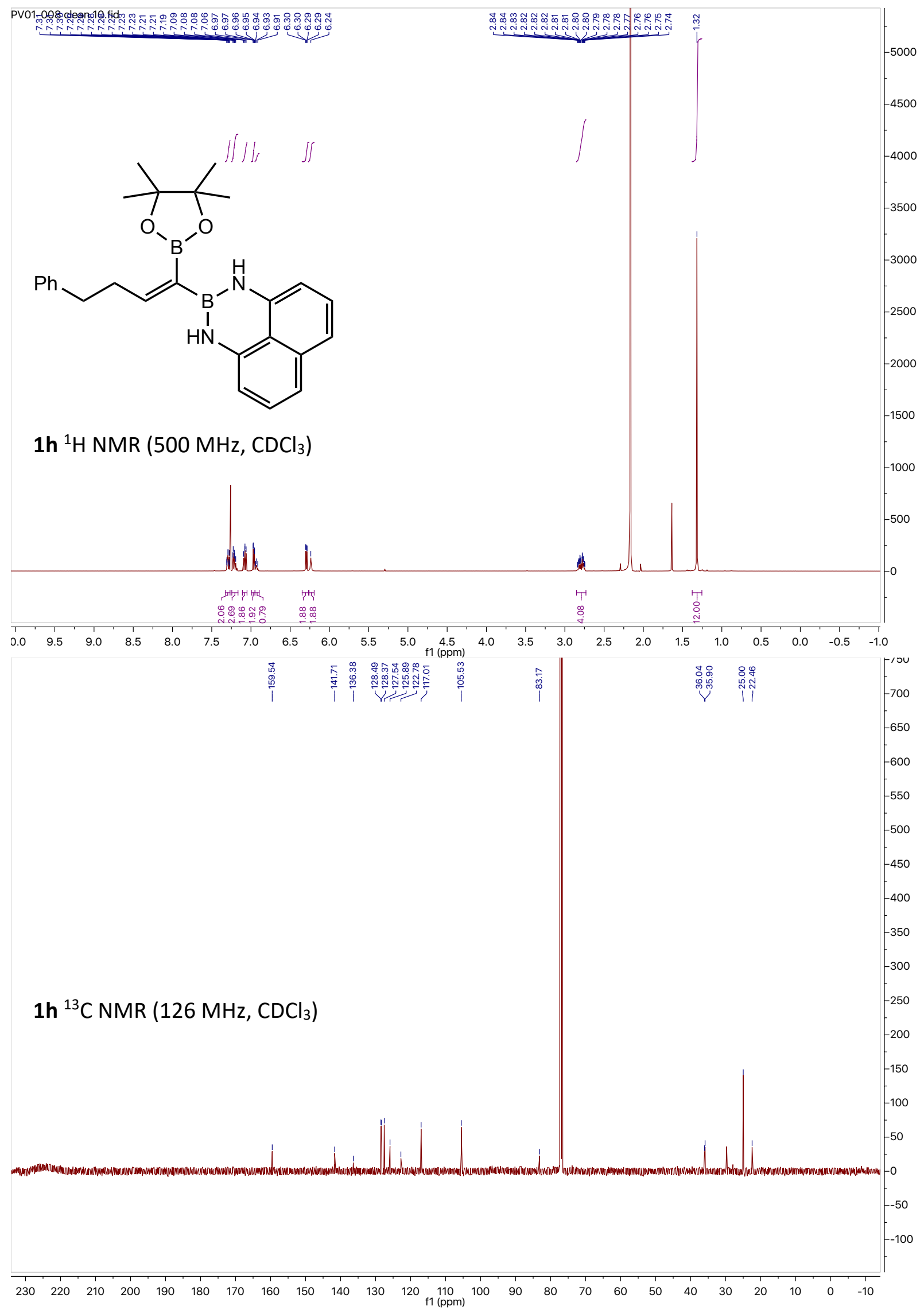




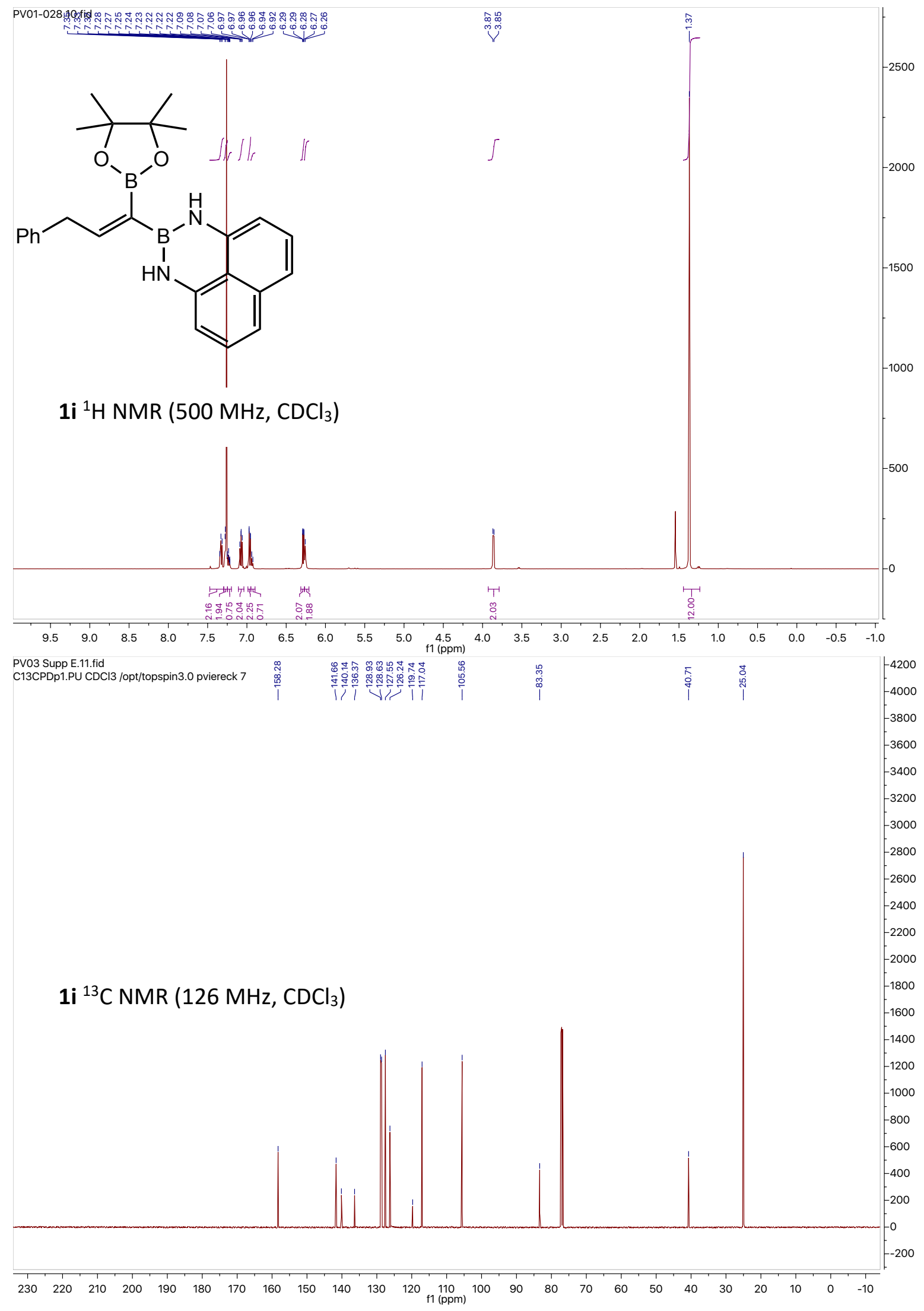




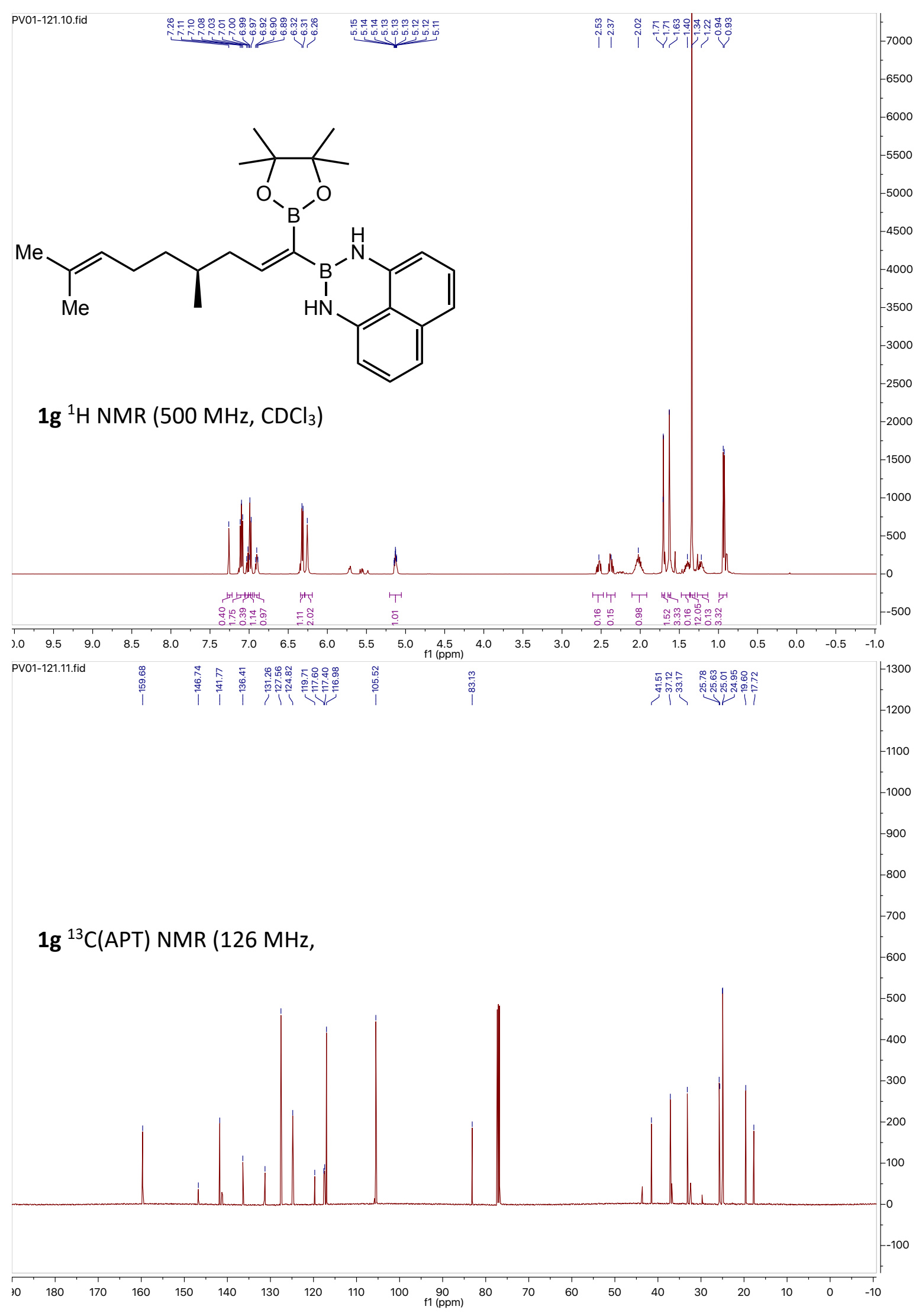




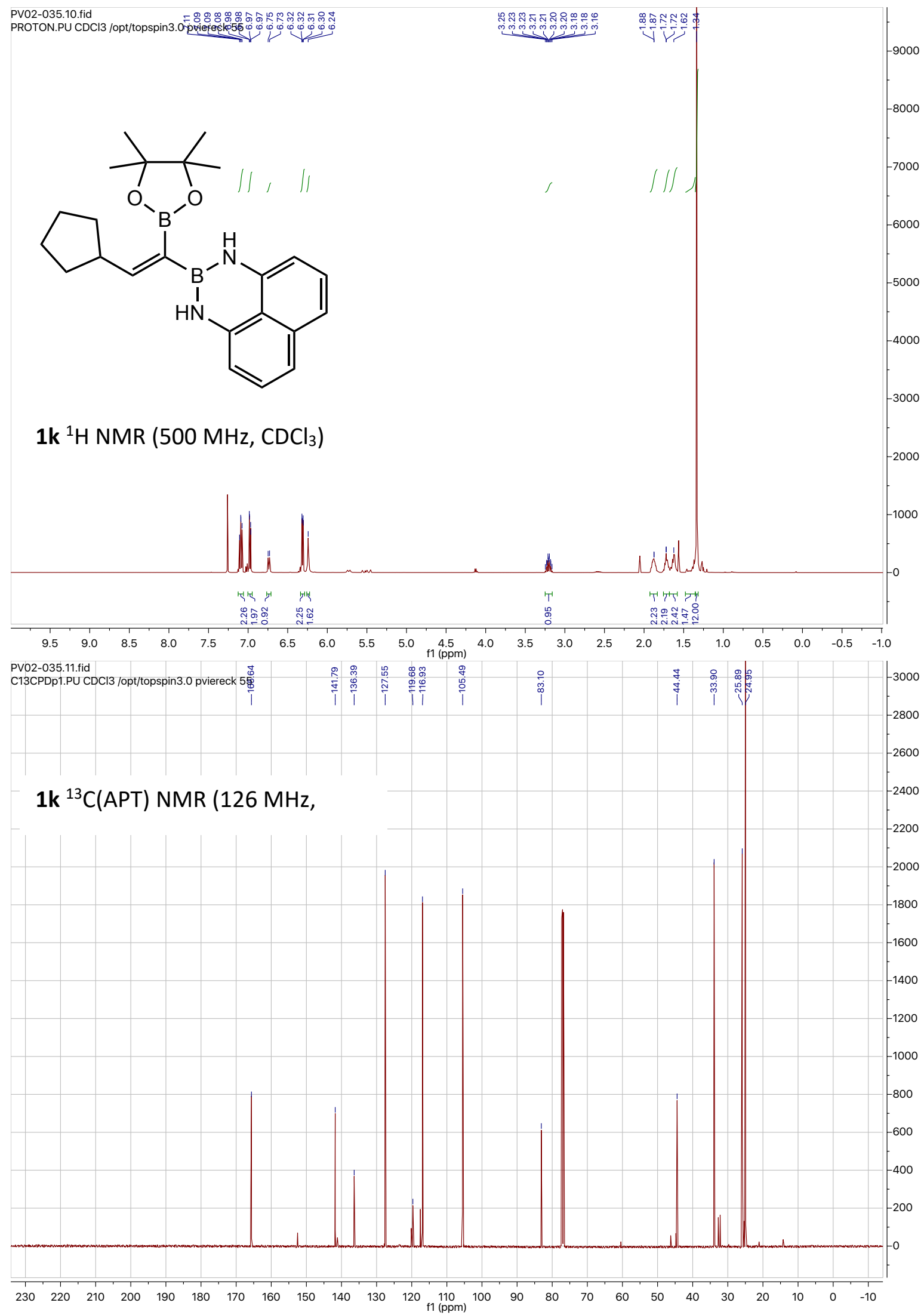




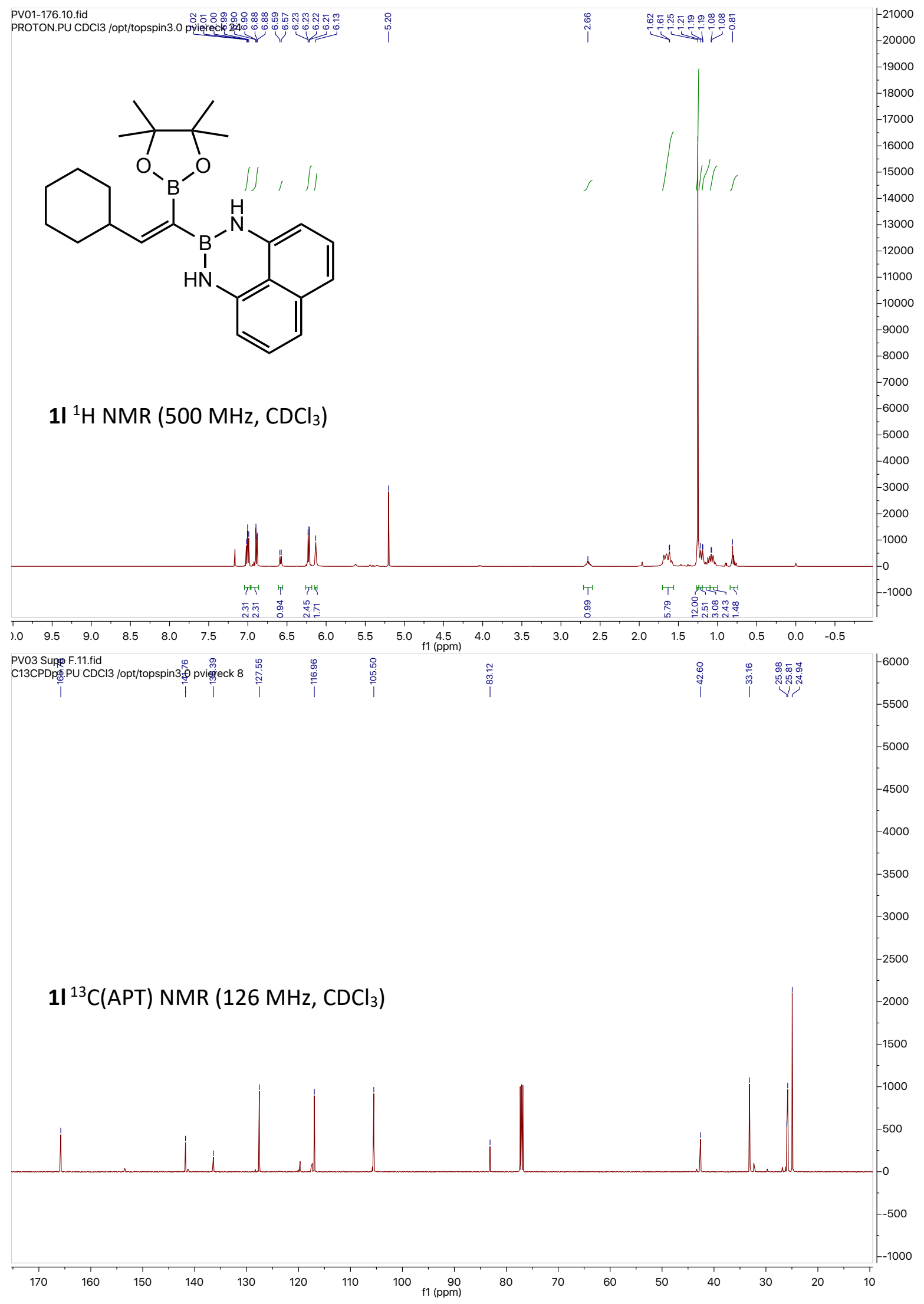




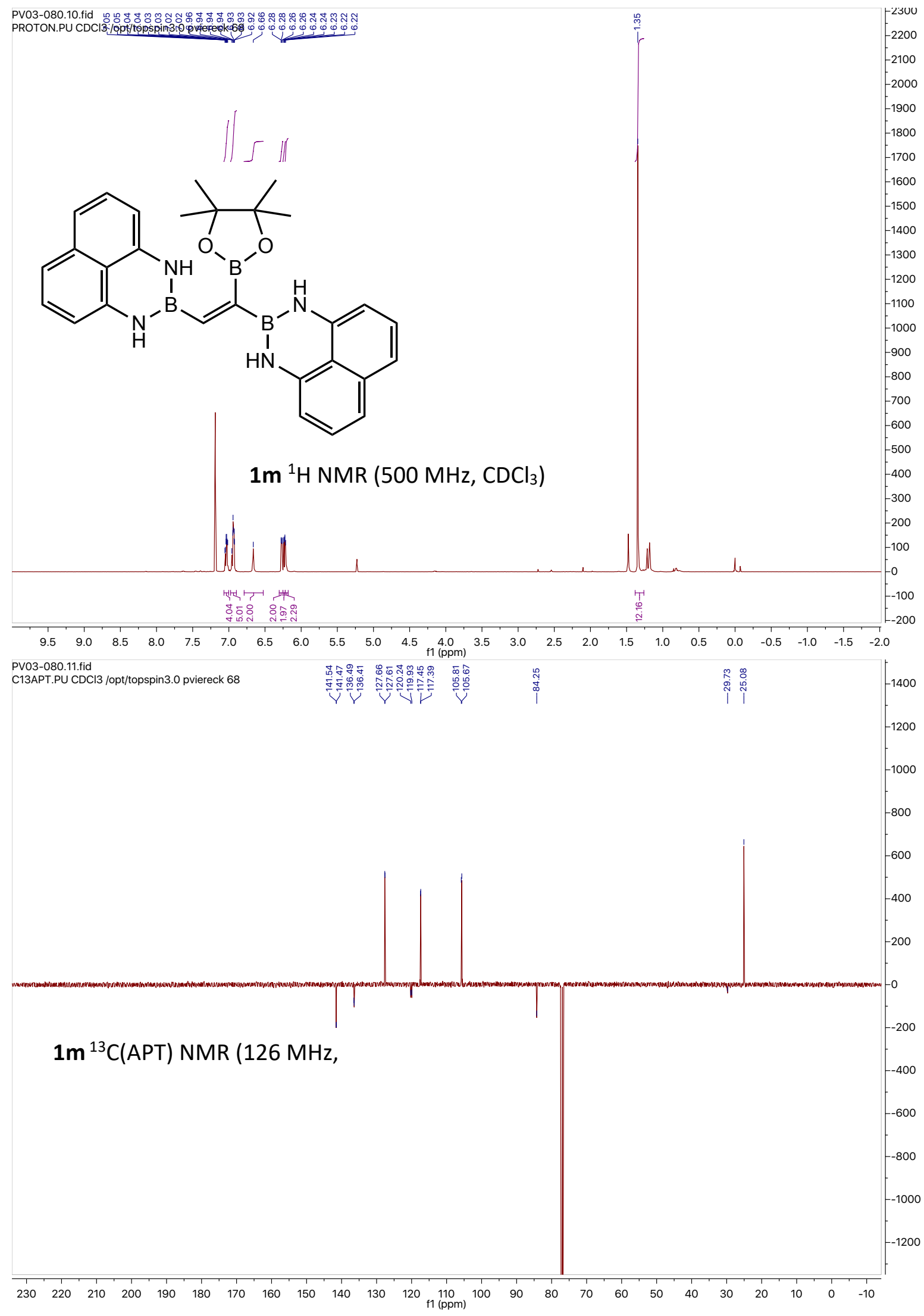




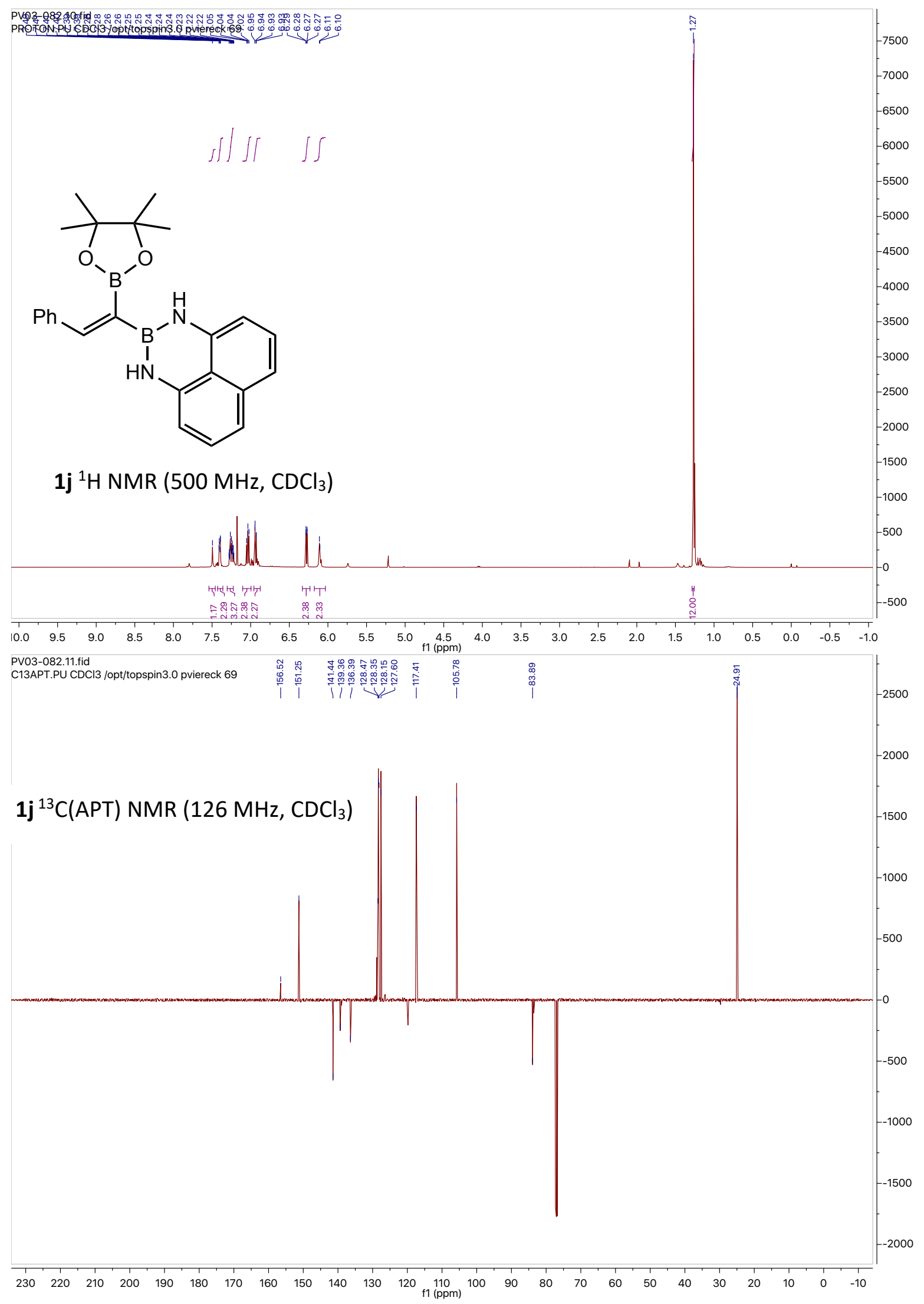




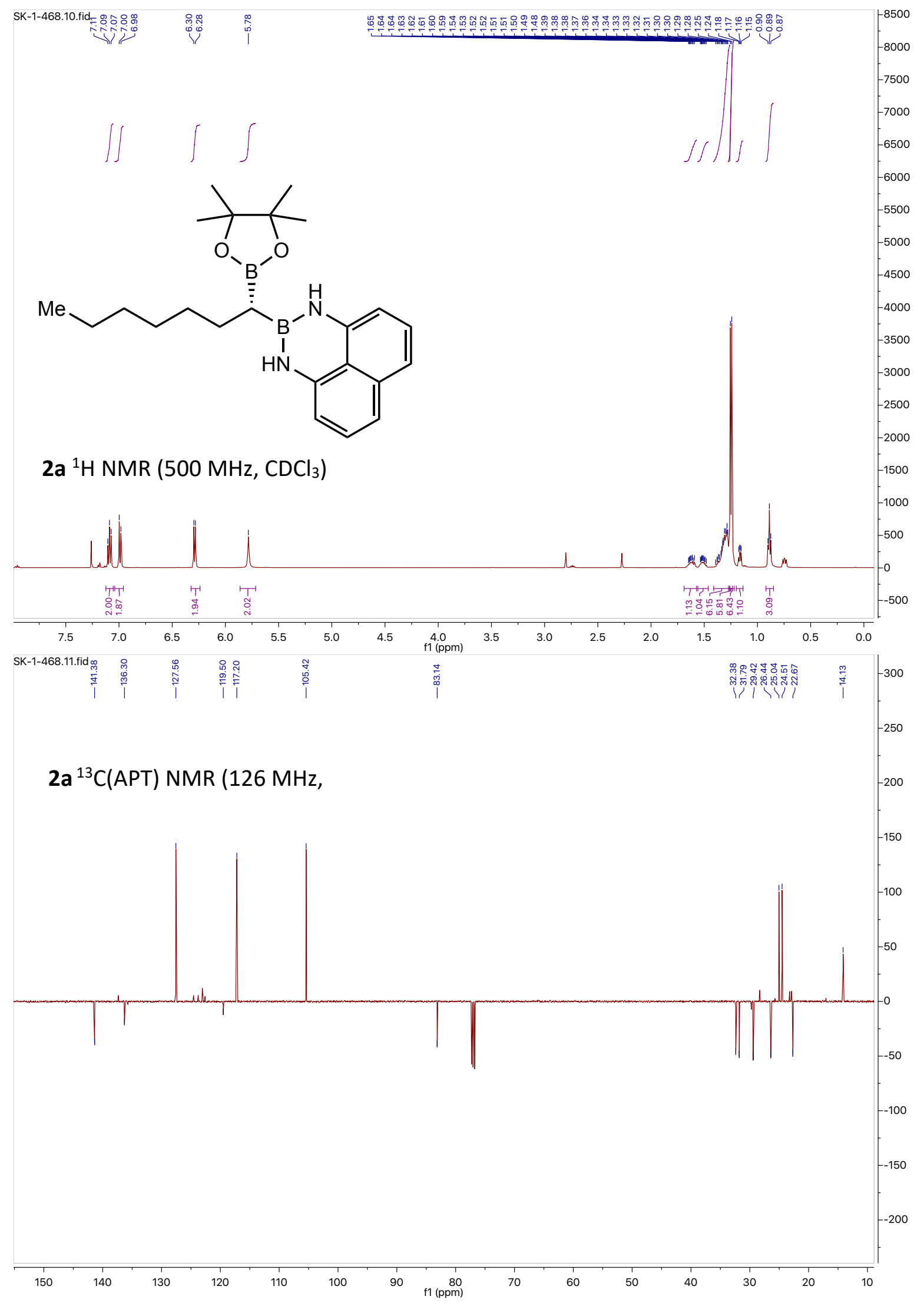




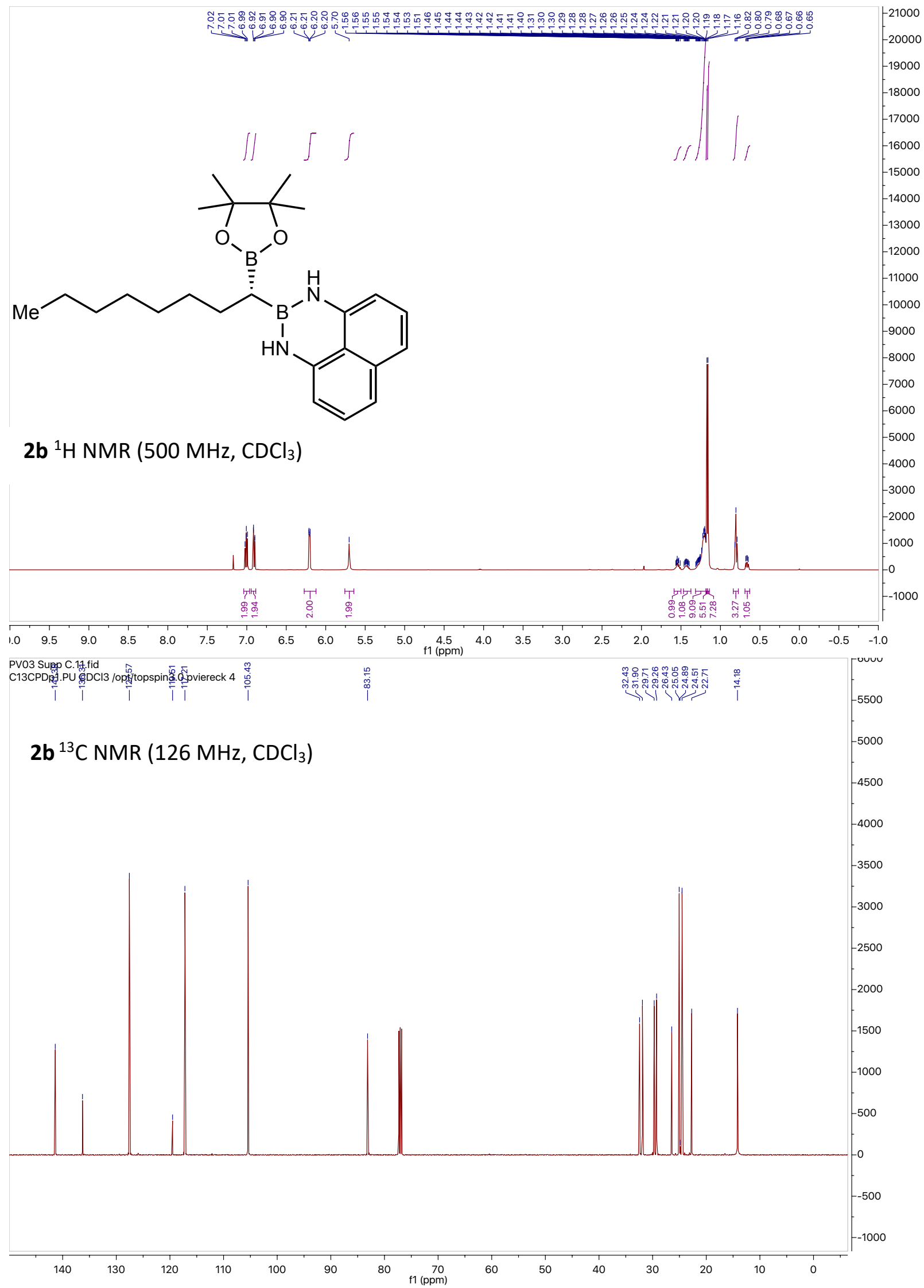




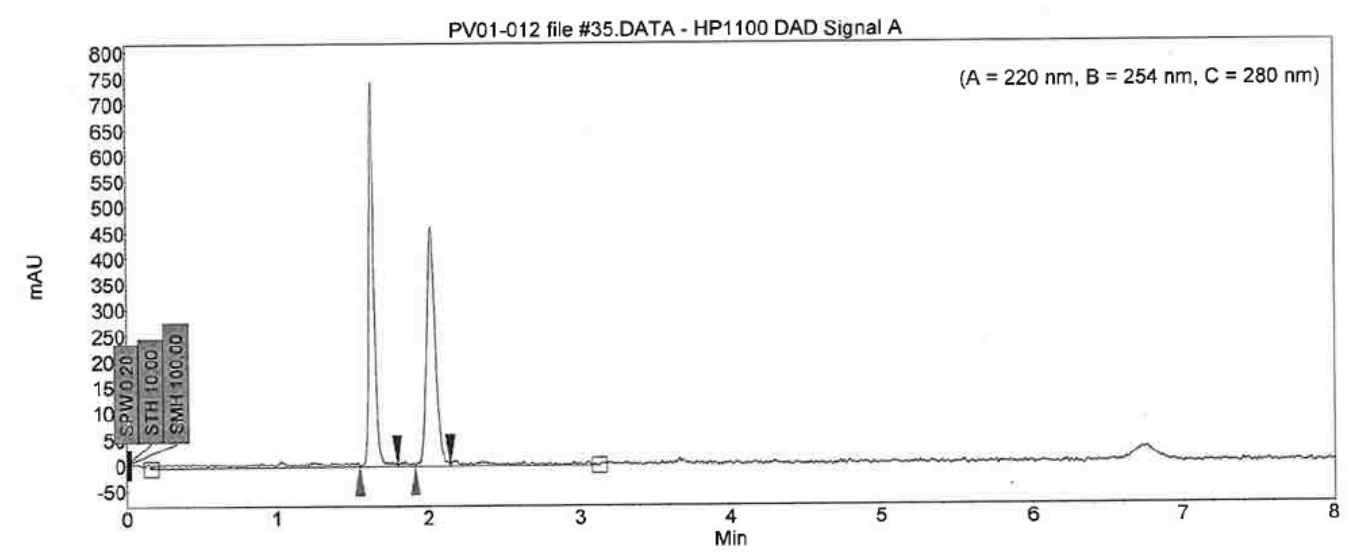

\begin{tabular}{|c|r|r|r|r|r|r|r|}
\hline Index & Time & Width & Height & Res. HW & Selectivity & Area & Area \\
\hline \hline & {$[$ IMin] } & {$[$ Min] } & {$[\mu \mathrm{\mu V}]$} & & & {$[\mu \mathrm{V}$. Min] } & {$[\%]$} \\
\hline 1 & 1.63 & 0.04 & 745.4 & 0.00 & 0.00 & 31.9 & 49.858 \\
\hline 2 & 2.02 & 0.06 & 464.7 & 4.55 & 1.24 & 32.1 & 50.142 \\
\hline & & & & & & & \\
\hline Total & & & 1210.1 & & & 64.1 & 100.000 \\
\hline
\end{tabular}

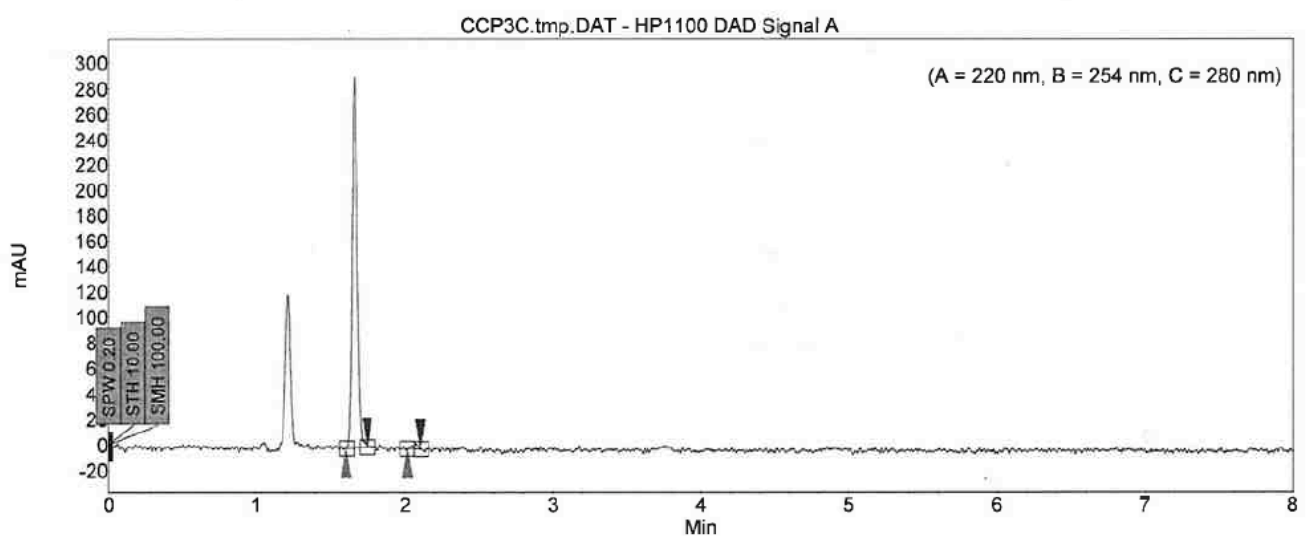

\begin{tabular}{|c|r|r|r|r|r|r|r|}
\hline Index & Time & Width & Height & Res. HW & Selectivity & Area & Area \\
\hline \hline & {$[$ Min] } & {$[$ Min] } & {$[\mu \mathrm{V}]$} & & & [jV.Min] & {$[\%]$} \\
\hline 1 & 1.66 & 0.04 & 291.7 & 0.00 & 0.00 & 11.9 & 98.544 \\
\hline 2 & 2.08 & 0.02 & 4.8 & 8.83 & 1.25 & 0.2 & 1.456 \\
\hline & & & & & & & \\
\hline Total & & & 296.5 & & & 12.1 & 100.000 \\
\hline
\end{tabular}



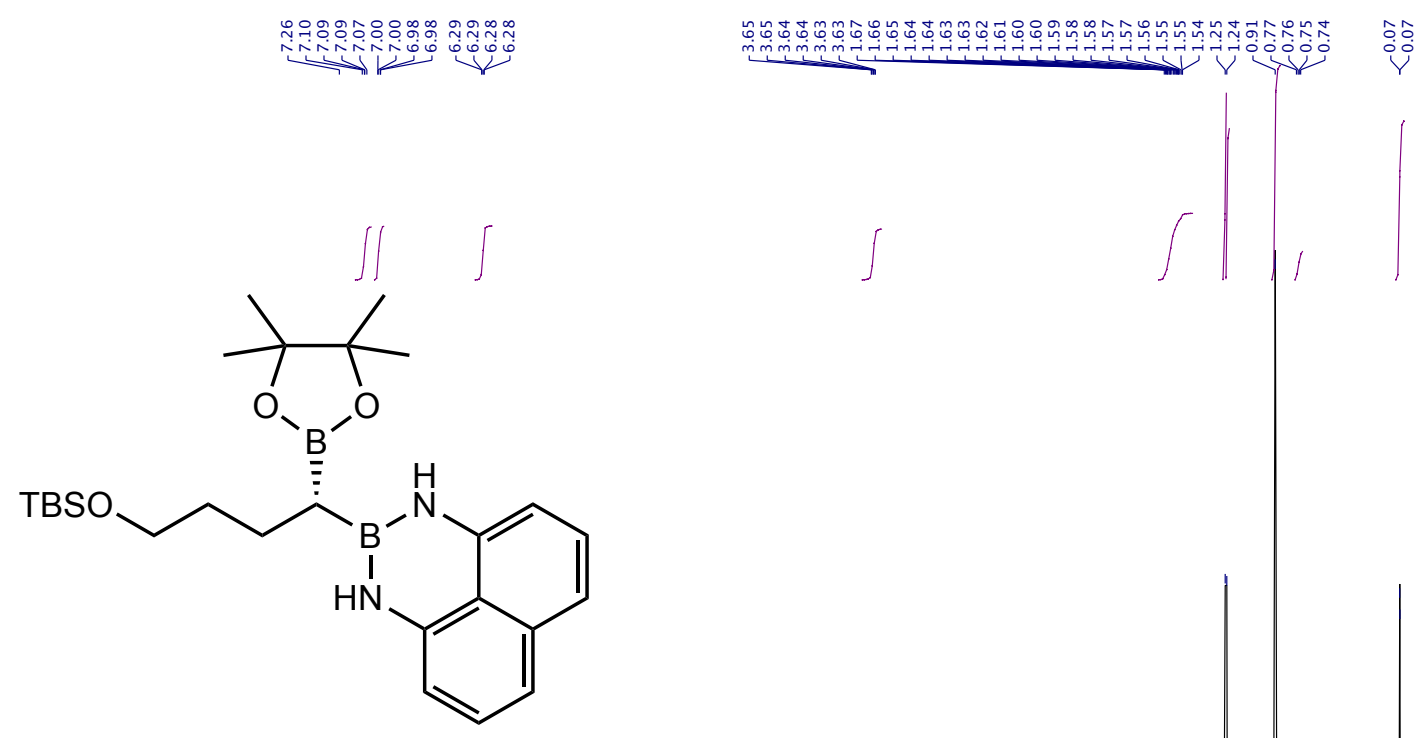

2c ${ }^{1} \mathrm{H}$ NMR $\left(500 \mathrm{MHz}, \mathrm{CDCl}_{3}\right)$

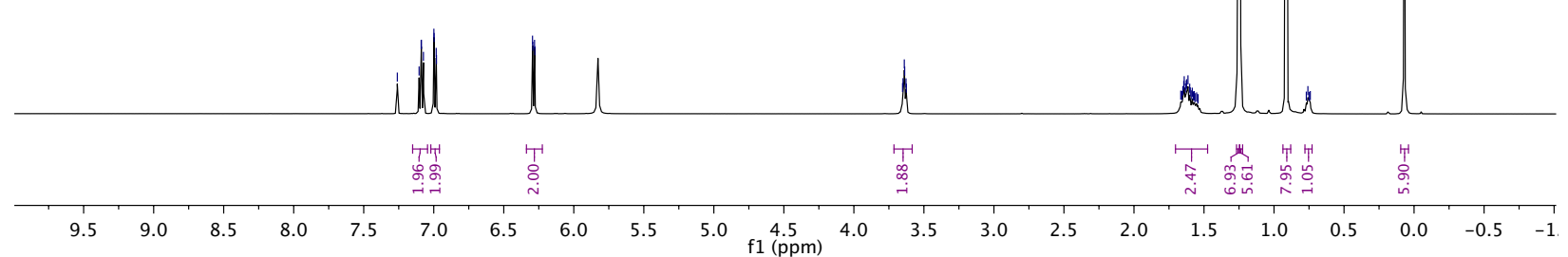

sk-1-466-column-A2.11.fid
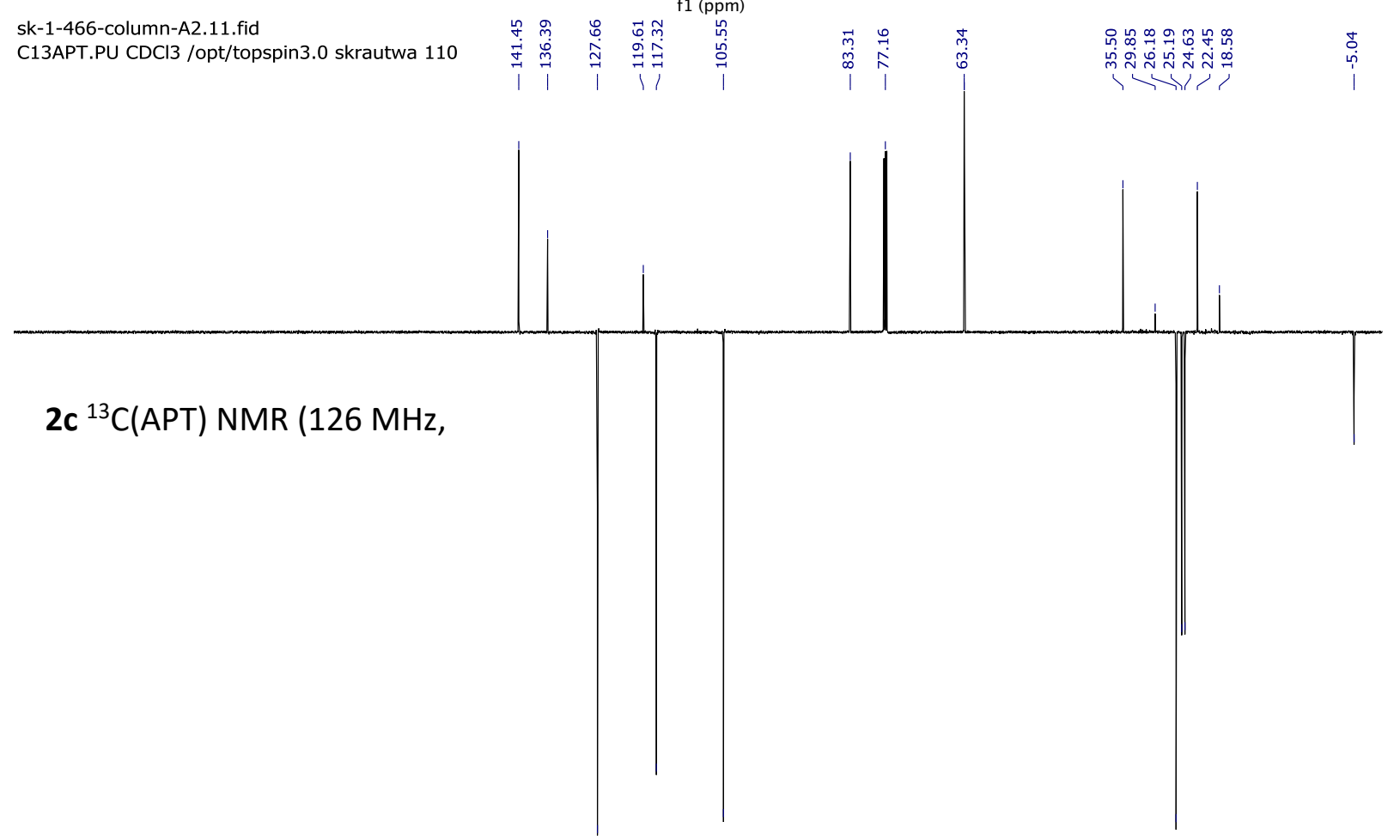

$\begin{array}{lllllllllllllllllllllllllllll}30 & 220 & 210 & 200 & 190 & 180 & 170 & 160 & 150 & 140 & 130 & 120 & \begin{array}{c}110 \\ \mathrm{f} 1\end{array}(\mathrm{ppm}) & 100 & 90 & 80 & 70 & 60 & 50 & 40 & 30 & 20 & 10 & 0 & -\end{array}$ 
SK-1-473 rac file\#0.DATA - HP1100 DAD Signal A

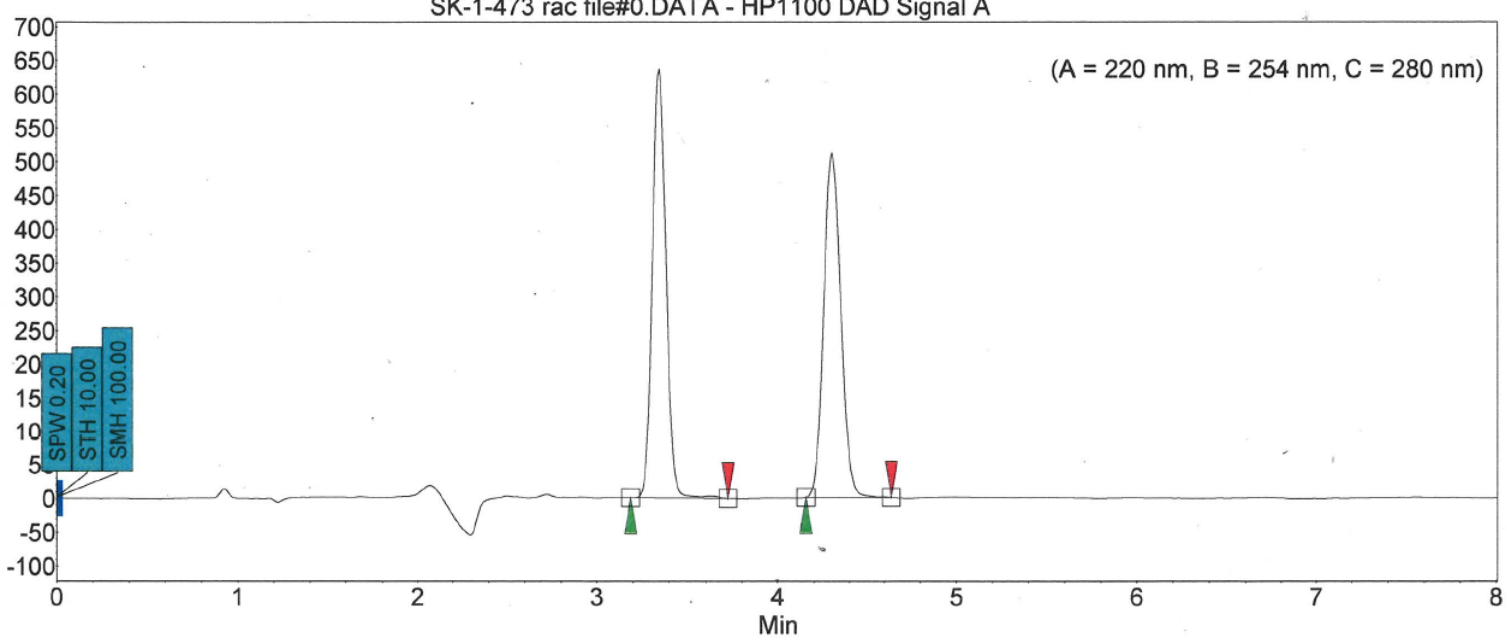

SK-1-466 ent file\#2.DATA - HP1100 DAD Signal A

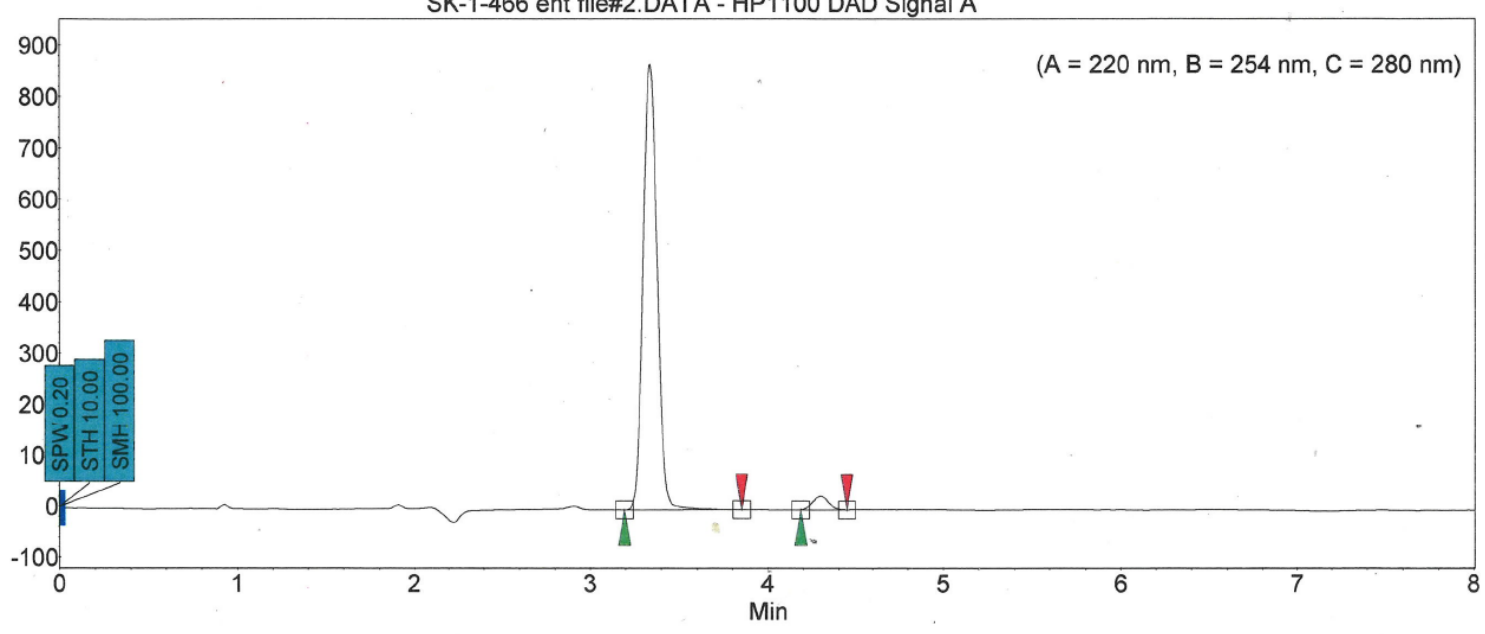

\begin{tabular}{|c|l|r|r|r|r|r|r|r|r|}
\hline Index & Name & Start & Time & End & RT Offset & Quantity & Height & Area & Area \\
\hline \hline & & {$[\mathrm{Min}]$} & {$[\mathrm{Min}]$} & {$[\mathrm{Min}]$} & {$[\mathrm{Min}]$} & {$[\%$ Area $]$} & {$[\mu \mathrm{V}]$} & {$[\mu \mathrm{V} . \mathrm{Min}]$} & {$[\%]$} \\
\hline 1 & UNKNOWN & 3.19 & 3.34 & 3.85 & 0.00 & 96.65 & 870.1 & 80.4 & 96.654 \\
\hline 2 & UNKNOWN & 4.18 & 4.29 & 4.45 & 0.00 & 3.35 & 26.9 & 2.8 & 3.346 \\
\hline & & & & & & & & & \\
\hline Total & & & & & & 100.00 & 896.9 & 83.2 & 100.000 \\
\hline
\end{tabular}




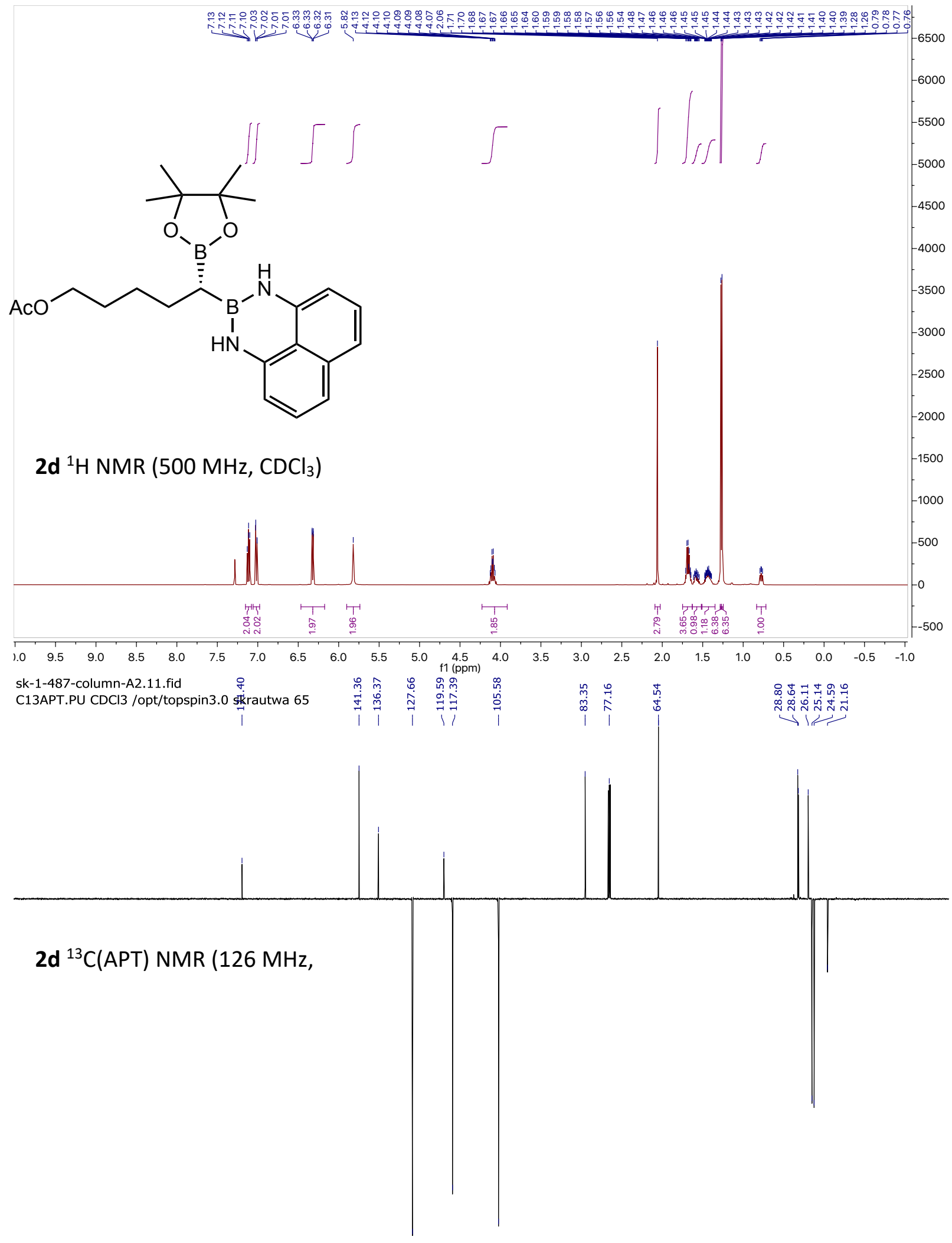

$\begin{array}{llllllllllllllllllllllllllllll}30 & 220 & 210 & 200 & 190 & 180 & 170 & 160 & 150 & 140 & 130 & 120 & 110 & 100 & 90 & 80 & 70 & 60 & 50 & 40 & 30 & 20 & 10 & 0 & -:\end{array}$ 


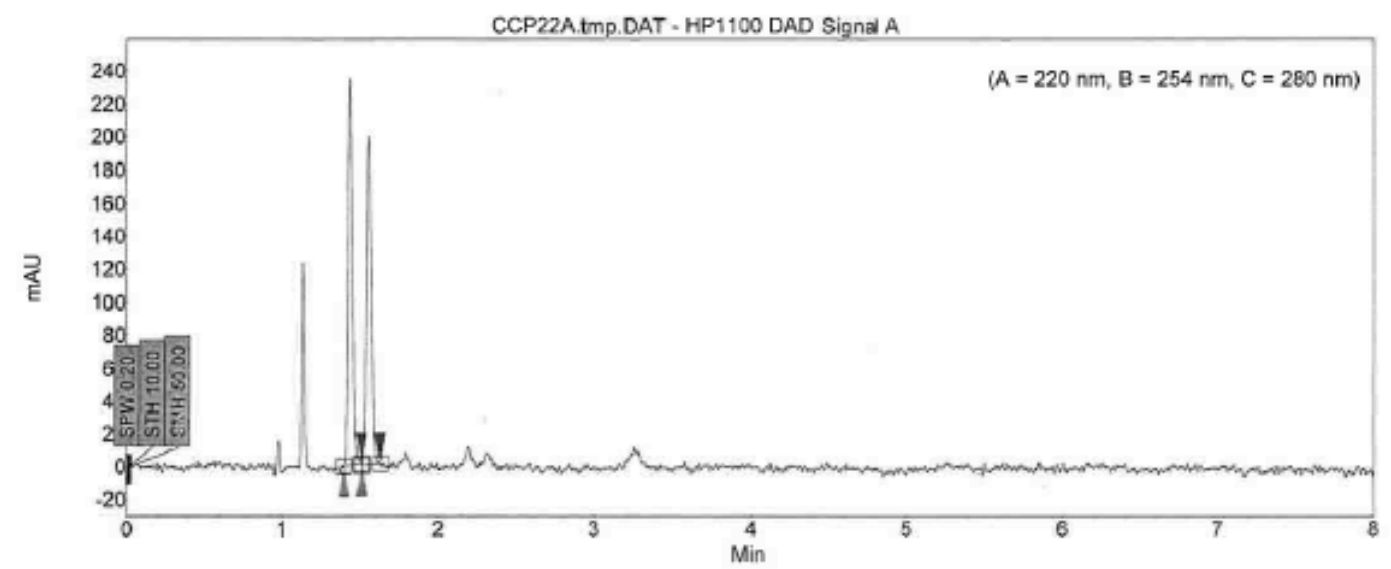

\begin{tabular}{|c|c|c|c|c|c|c|c|}
\hline Index & Time & Width & Height & Ros. HW & Selectivity & Area & Area \\
\hline & {$[\mathrm{Min]}$} & 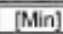 & [uv] & & & [uV.Min] & {$[\%]$} \\
\hline 1 & 1.44 & 0.03 & 234.1 & 0.00 & 0.00 & 7.7 & 50.218 \\
\hline 2 & 1.56 & 0.04 & 198.9 & 2.11 & 1.08 & 7.6 & 49.782 \\
\hline Tota & & & 4330 & & & 15.3 & 100,000 \\
\hline
\end{tabular}

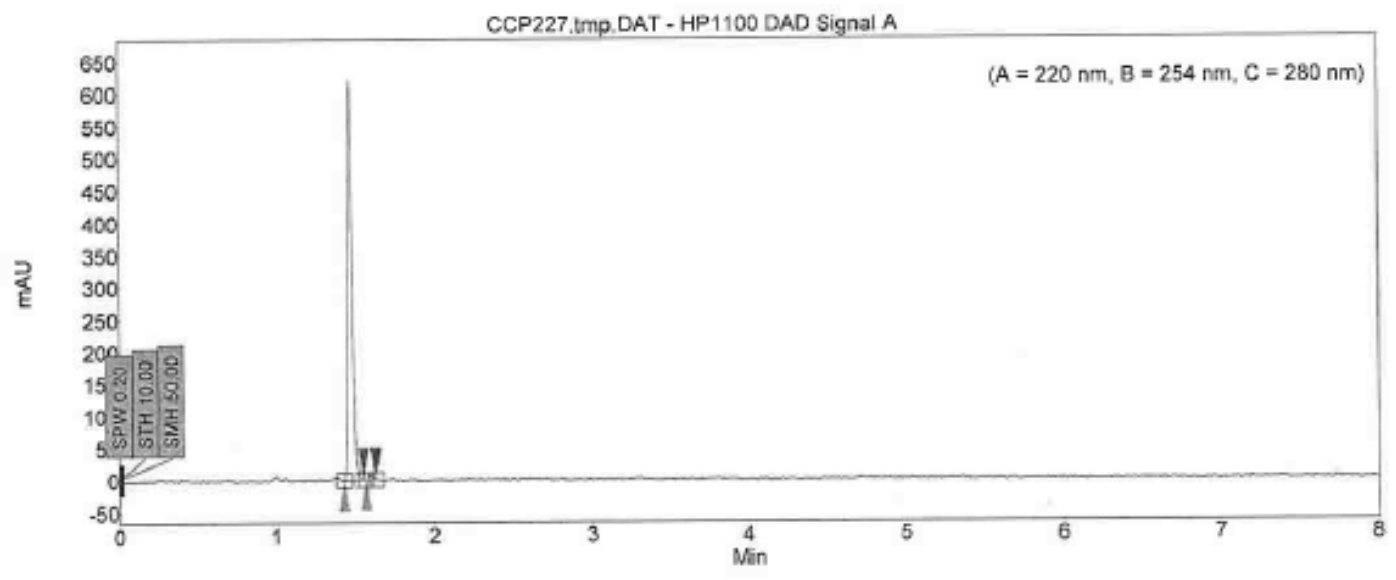

\begin{tabular}{|c|r|r|r|r|r|r|r|}
\hline Index & Time & Width & Height & Res. HW & Selectivity & Area & Area \\
\hline \hline & [Min] & {$[$ Min] } & {$[\mu \mathrm{V}]$} & & & {$[\mu \mathrm{V}$. Min] } & {$[\%]$} \\
\hline 1 & 1.47 & 0.03 & 621.7 & 0.00 & 0.00 & 20.1 & 98.725 \\
\hline 2 & 1.59 & 0.03 & 8.8 & 2.55 & 1.08 & 0.3 & 1.275 \\
\hline & & & & & & & \\
\hline Total & & & 630.5 & & & 20.4 & 100.000 \\
\hline
\end{tabular}


<smiles>CC1(C)OB([C@@H](CCCOC2CCCCO2)B2Nc3cccc4cccc(c34)N2)OC1(C)C</smiles>

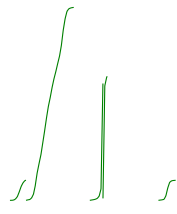

$2 \mathrm{e}^{1} \mathrm{H} \mathrm{NMR}\left(500 \mathrm{MHz}, \mathrm{CDCl}_{3}\right)$

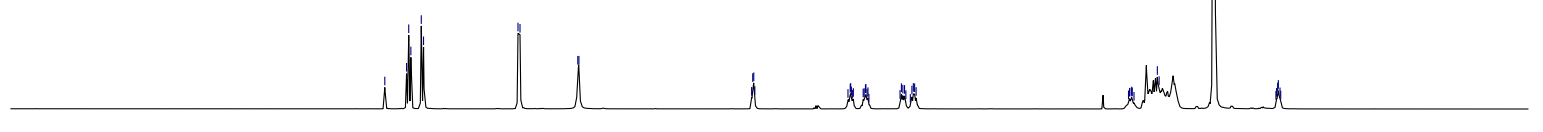

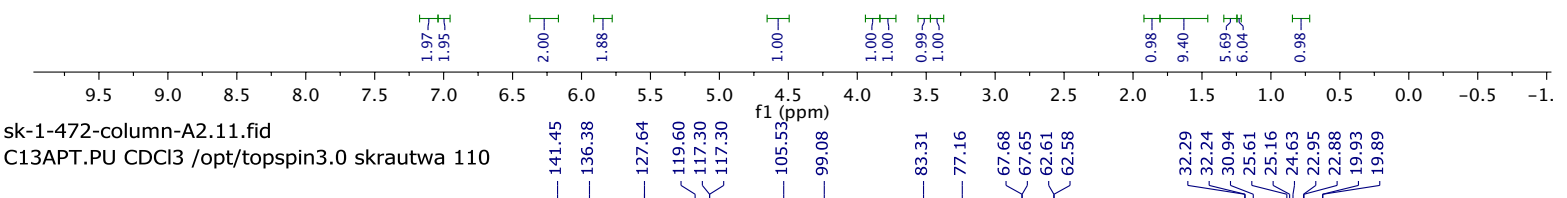

\section{$2 e^{13} \mathrm{C}(\mathrm{APT}) \mathrm{NMR}(126 \mathrm{MHz}$,}
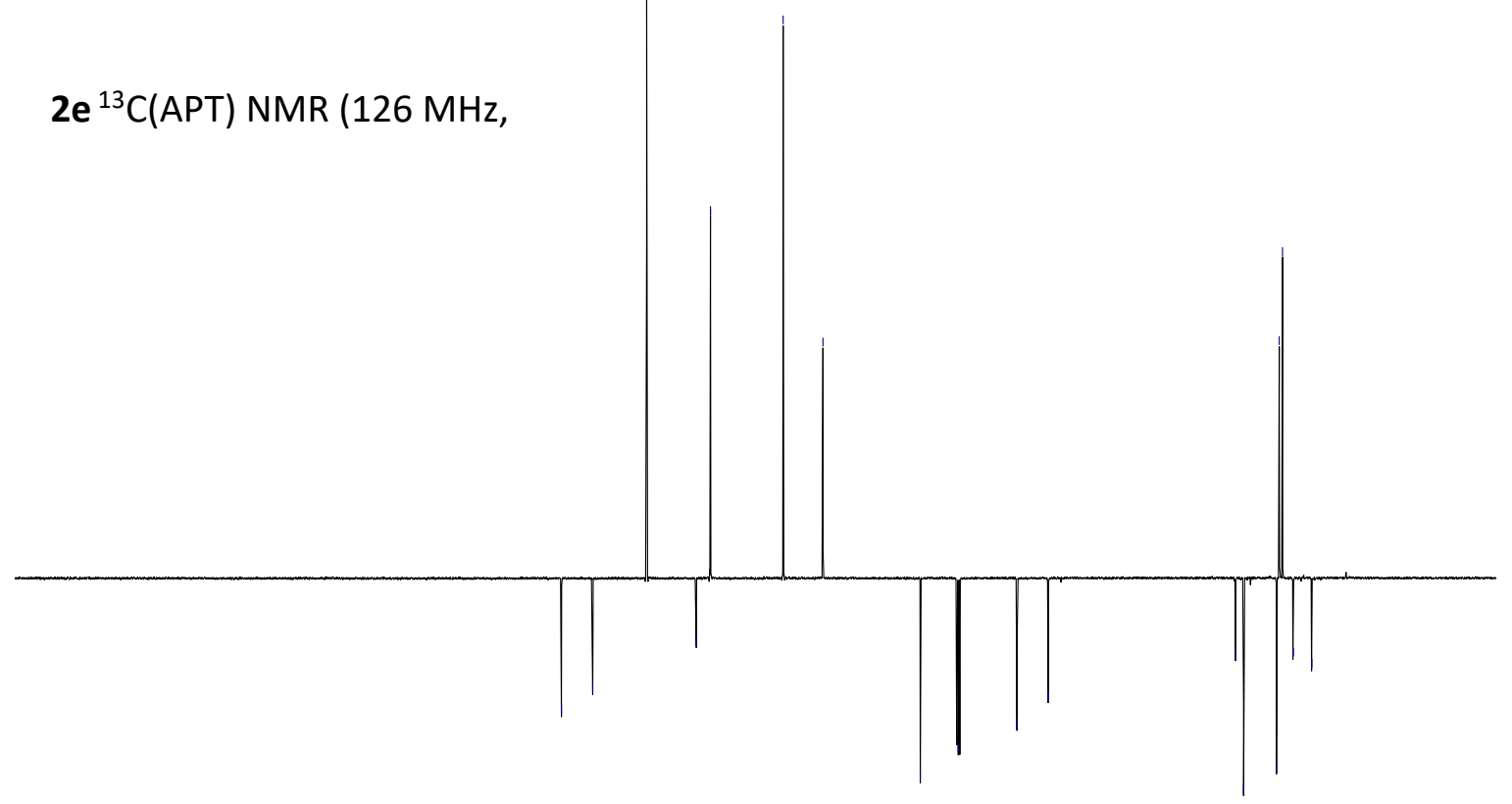

$\begin{array}{llllllllllllllllllllllllll}30 & 220 & 210 & 200 & 190 & 180 & 170 & 160 & 150 & 140 & 130 & 120 & 110 & 100 & 90 & 80 & 70 & 60 & 50 & 40 & 30 & 20 & 10 & 0 & -:\end{array}$ 


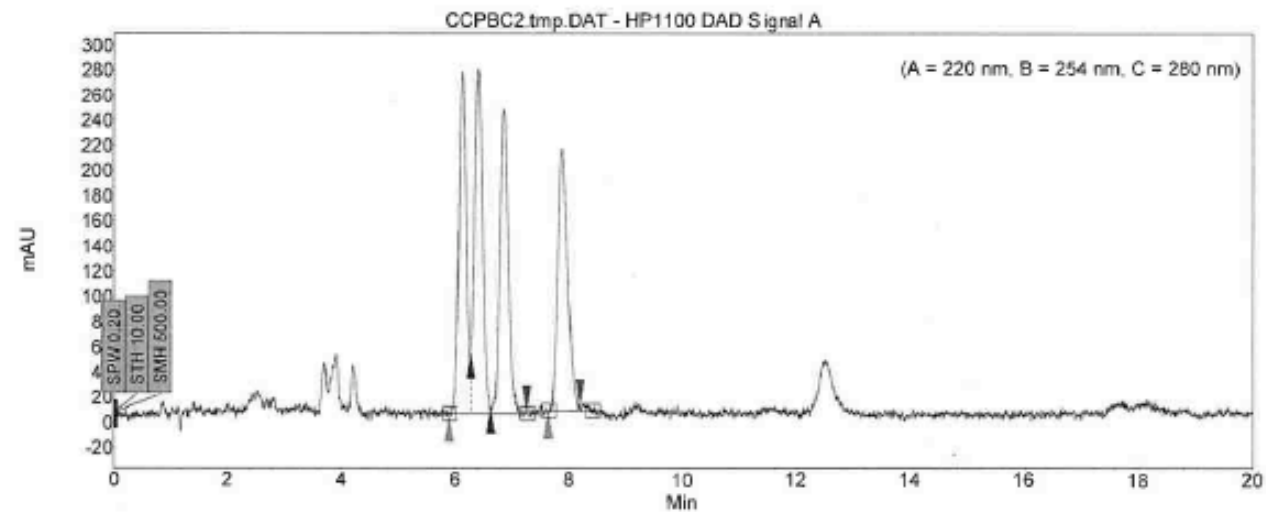

\begin{tabular}{|c|r|r|r|r|r|r|r|}
\hline Index & Time & Width & Height & Res. HW & Seleclivily & Area & Area \\
\hline \hline & {$[$ Min] } & {$[$ Min] } & {$[\mu \mathrm{H}]$} & & & {$[$ [u.Min] } & {$[\%]$} \\
\hline 1 & 6.13 & 0.14 & 272.2 & 0.00 & 0.00 & 41.4 & 24.046 \\
\hline 3 & 6.41 & 0.15 & 274.4 & 1.14 & 1.05 & 44.0 & 25.547 \\
\hline 2 & 6.85 & 0.16 & 242.2 & 1.66 & 1.07 & 43.6 & 25.314 \\
\hline 4 & 7.89 & 0.19 & 208.2 & 3.40 & 1.15 & 43.2 & 25.093 \\
\hline & & & & & & & \\
\hline Total & & & 997.1 & & & 172.3 & 100.000 \\
\hline
\end{tabular}

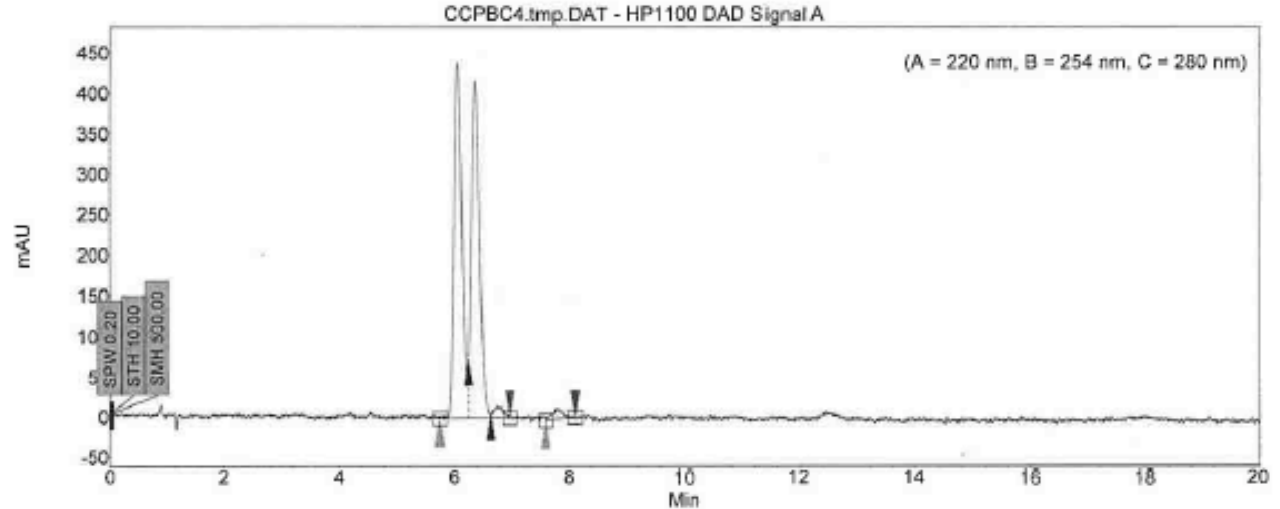

\begin{tabular}{|c|r|r|r|r|r|r|r|}
\hline Index & Time & Width & Height & Res. HW & Selectivity & Area & Area \\
\hline & [Min] & {$[$ Min] } & {$[\mu \mathrm{N}]$} & & & [uV.Min] & {$[\%]$} \\
\hline 1 & 6.05 & 0.14 & 439.2 & 0.00 & 0.00 & 69.4 & 48.331 \\
\hline 2 & 6.36 & 0.16 & 416.1 & 1.21 & 1.05 & 68.9 & 48.001 \\
\hline 3 & 6.75 & 0.13 & 15.8 & 1.55 & 1.05 & 2.6 & 1.839 \\
\hline 4 & 7.76 & 0.15 & 13.3 & 4.15 & 1.15 & 2.6 & 1.829 \\
\hline & & & & & & & \\
\hline Total & & & 884.4 & & & 143.6 & 100.000 \\
\hline
\end{tabular}

$91 \% 20$ 


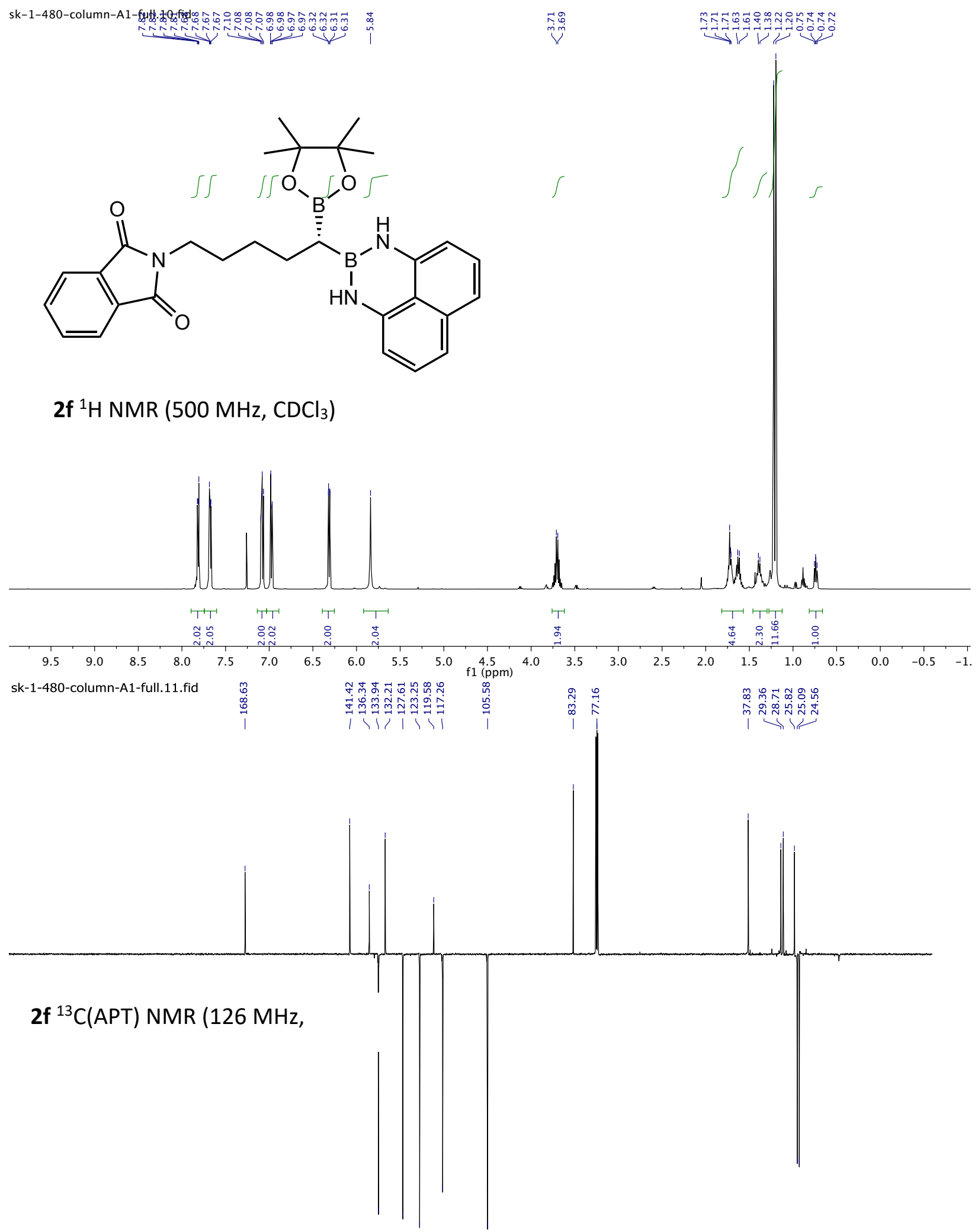

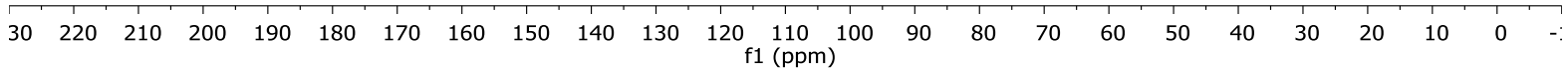



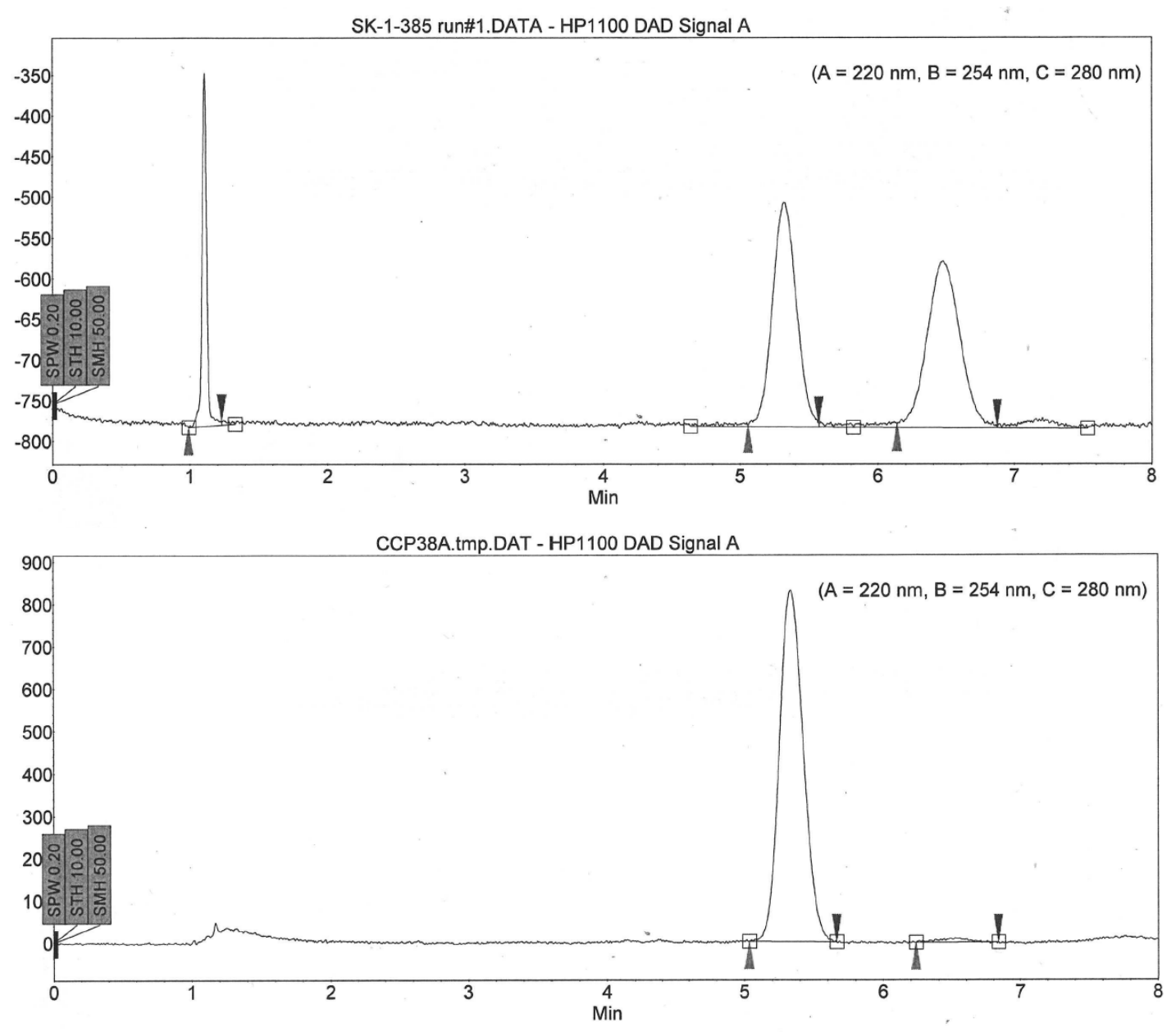

\begin{tabular}{|c|r|r|r|r|r|r|r|}
\hline Index & Time & Width & Height & Res. HW & Selectivity & Area & Area \\
\hline \hline & {$[$ Min] } & {$[$ Min] } & {$[\mu \mathrm{V}]$} & & & {$[\mu \mathrm{V} . \mathrm{Min}]$} & {$[\%]$} \\
\hline 1 & 5.33 & 0.19 & 829.2 & 0.00 & 0.00 & 166.2 & 98.649 \\
\hline 2 & 6.52 & 0.16 & 10.3 & 4.07 & 1.22 & 2.3 & 1.351 \\
\hline & & & & & & & \\
\hline Total & & & 839.6 & & & 168.4 & 100.000 \\
\hline
\end{tabular}




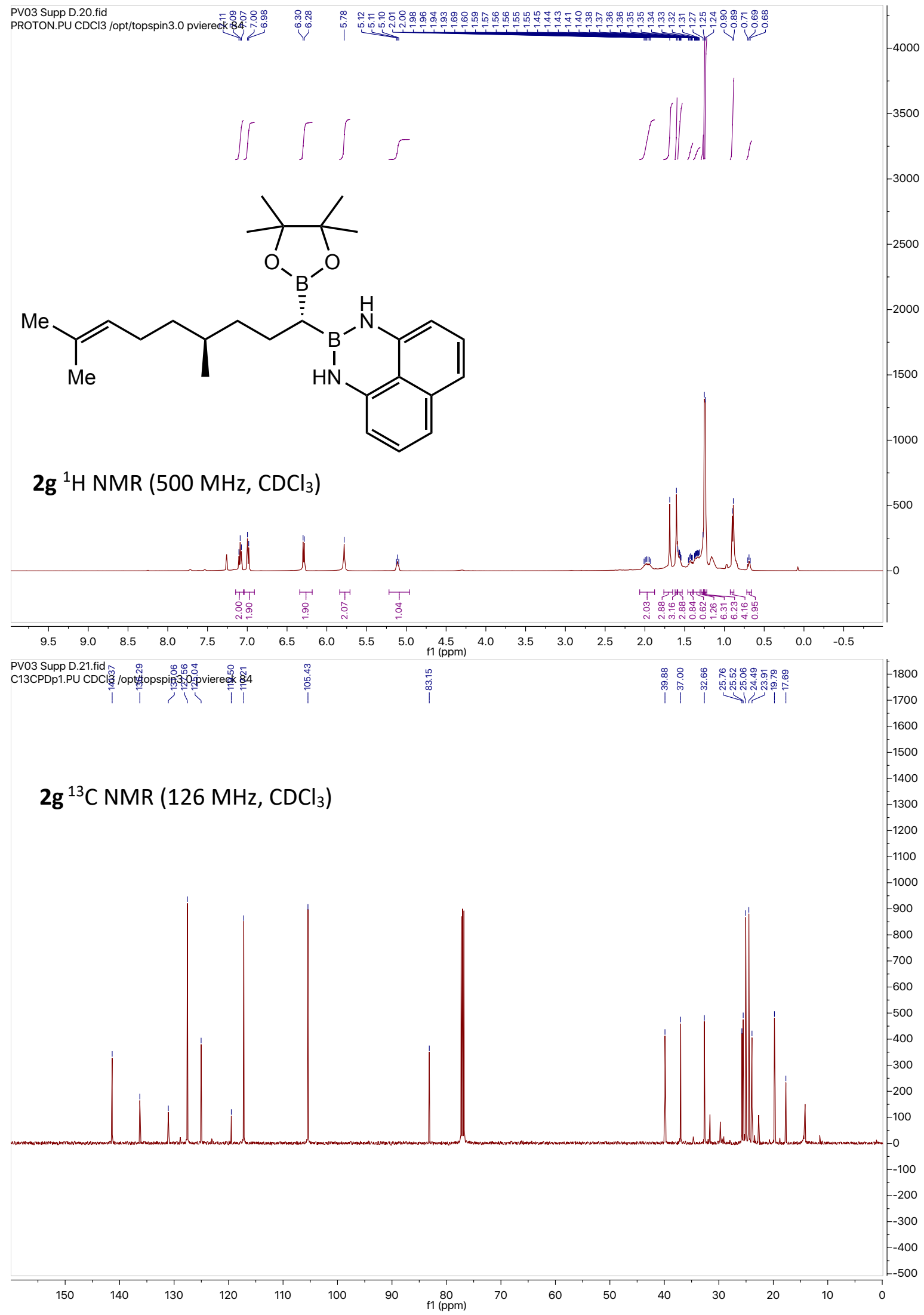




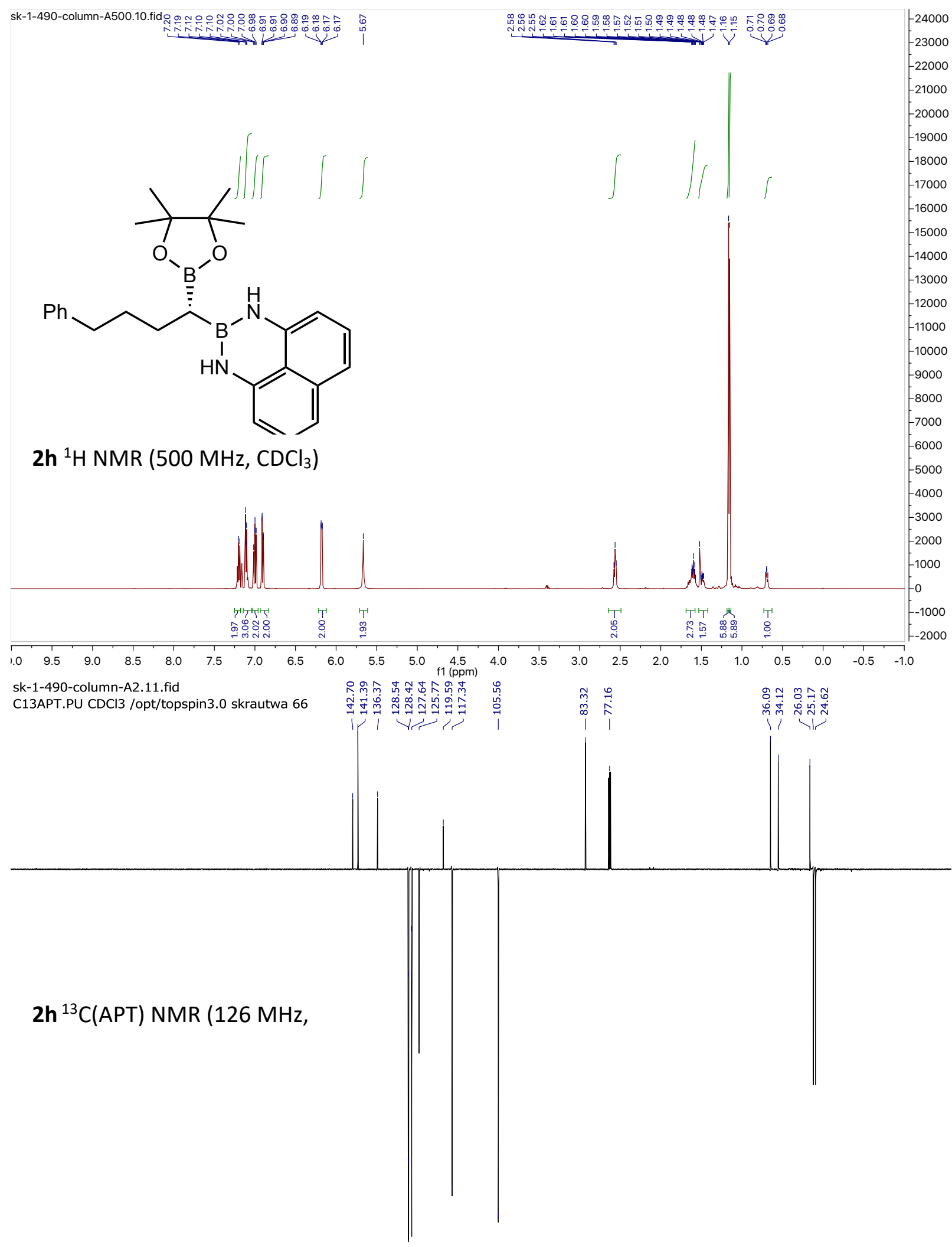

$\begin{array}{lllllllllllllllllllllllllll}30 & 220 & 210 & 200 & 190 & 180 & 170 & 160 & 150 & 140 & 130 & 120 & \begin{array}{c}110 \\ \mathrm{f} 1\end{array}(\mathrm{ppm}) & 100 & 90 & 80 & 70 & 60 & 50 & 40 & 30 & 20 & 10 & 0 & -\end{array}$ 


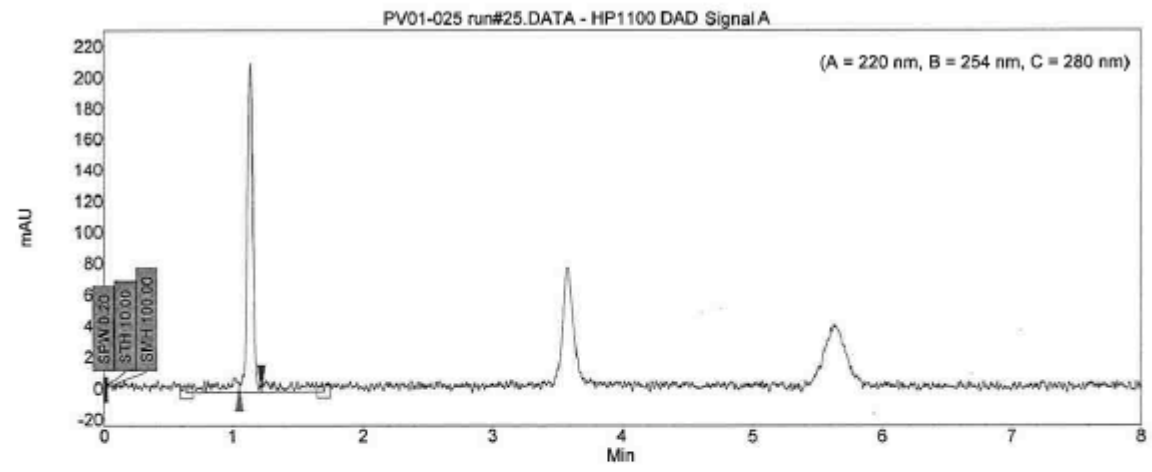

\begin{tabular}{|c|r|r|r|r|r|r|r|}
\hline Index & Time & Width & Height & Res. HW & Selectivity & Area & Area \\
\hline & {$[$ Min] } & {$[$ Min] } & {$[\mathrm{uM}]$} & & & {$[\mathrm{UV} . \mathrm{Min}]$} & {$[\%]$} \\
\hline 1 & 1.14 & 0.04 & 211.4 & 0.00 & 0.00 & 10.0 & 100.000 \\
\hline & & & & & & & \\
\hline Total & & & 211.4 & & & 10.0 & 100.000 \\
\hline
\end{tabular}

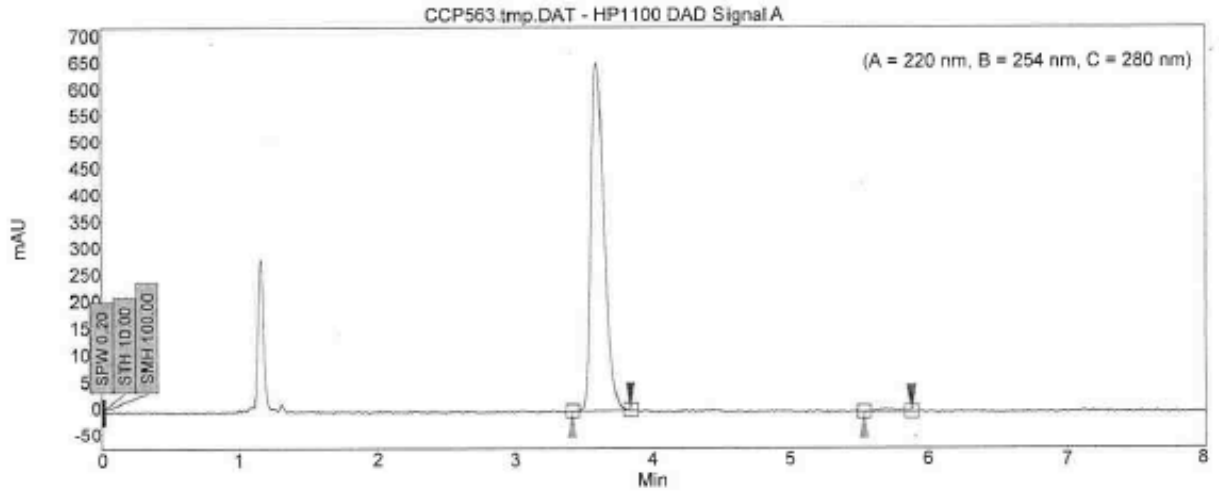

\begin{tabular}{|c|r|r|r|r|r|r|r|}
\hline Index & Time & Width & Height & Res. HW & Selectivity & Area & Area \\
\hline \hline & {$[$ Min] } & {$[$ Min] } & {$[\mathrm{\mu V}$ ] } & & & [uV.Min] & {$[\%]$} \\
\hline 1 & 3.60 & 0.10 & 654.6 & 0.00 & 0.00 & 71.4 & 98.483 \\
\hline 2 & 5.65 & 0.13 & 6.2 & 10.56 & 1.57 & 1.1 & 1.517 \\
\hline & & & & & & & \\
\hline Total & & & 660.8 & & & 72.5 & 100.000 \\
\hline
\end{tabular}




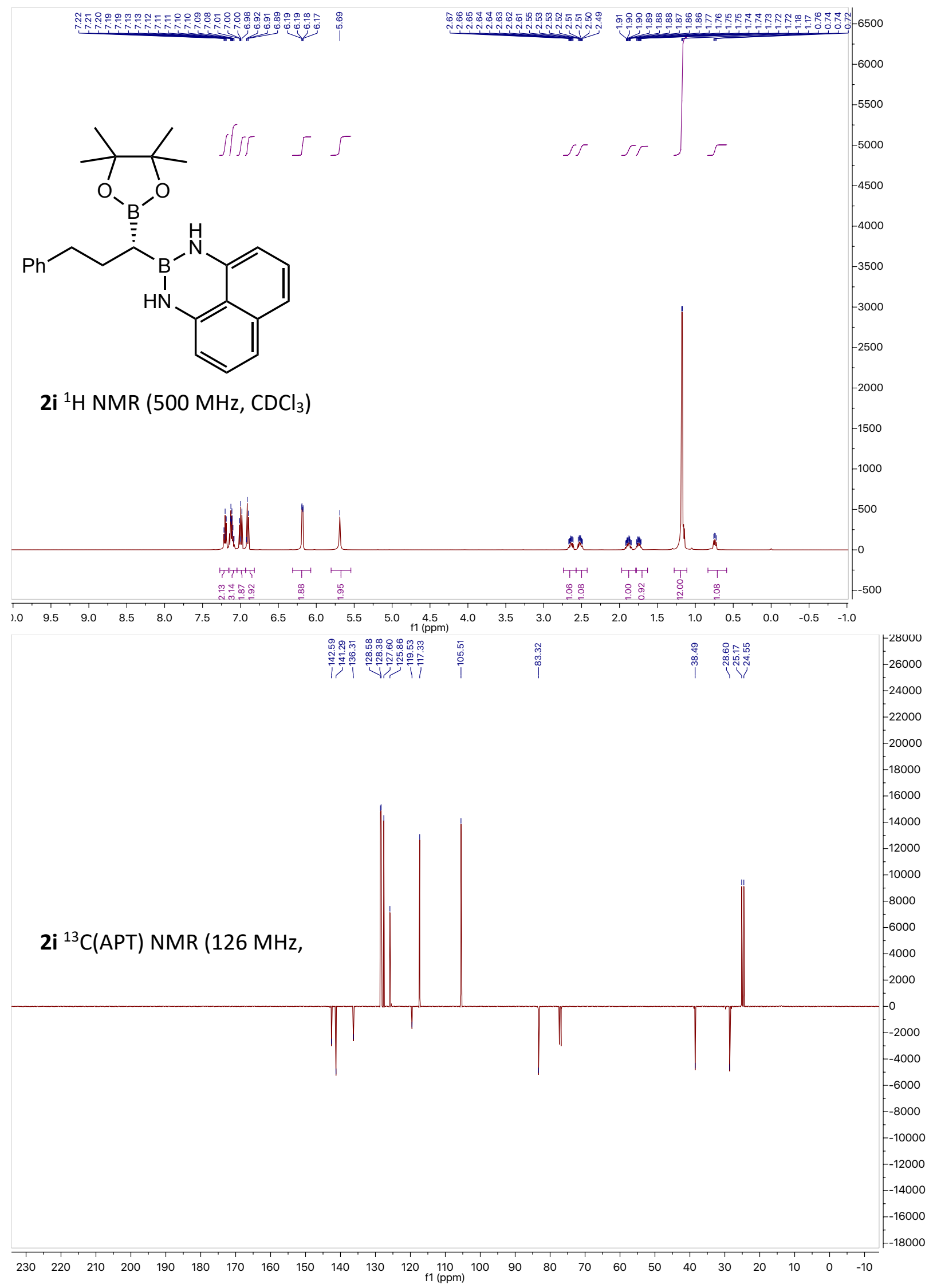


CCP110E.tmp.DAT - HP1100 DAD Signal A

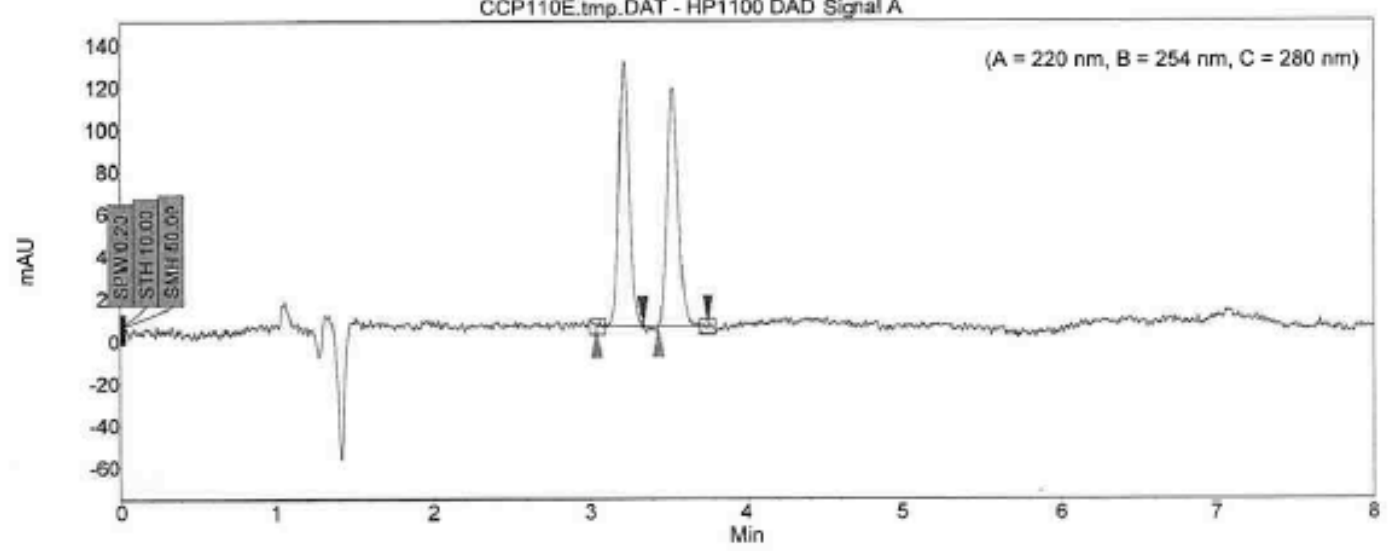

\begin{tabular}{|c|r|r|r|r|r|r|r|}
\hline Index & Time & Width & Heght & Res. HW & Seloctivity & Area & Area \\
\hline & [Min] & [Min] & [uV] & & & [uV.Min] & {$[\%]$} \\
\hline 1 & 3.22 & 0.07 & 125.2 & 0.00 & 0.00 & 9.7 & 50.282 \\
\hline 2 & 3.53 & 0.08 & 113.0 & 2.49 & 1.10 & 9.6 & 49.718 \\
\hline & & & & & & & \\
\hline Total & & & 238.3 & & & 19.2 & 100.000 \\
\hline
\end{tabular}

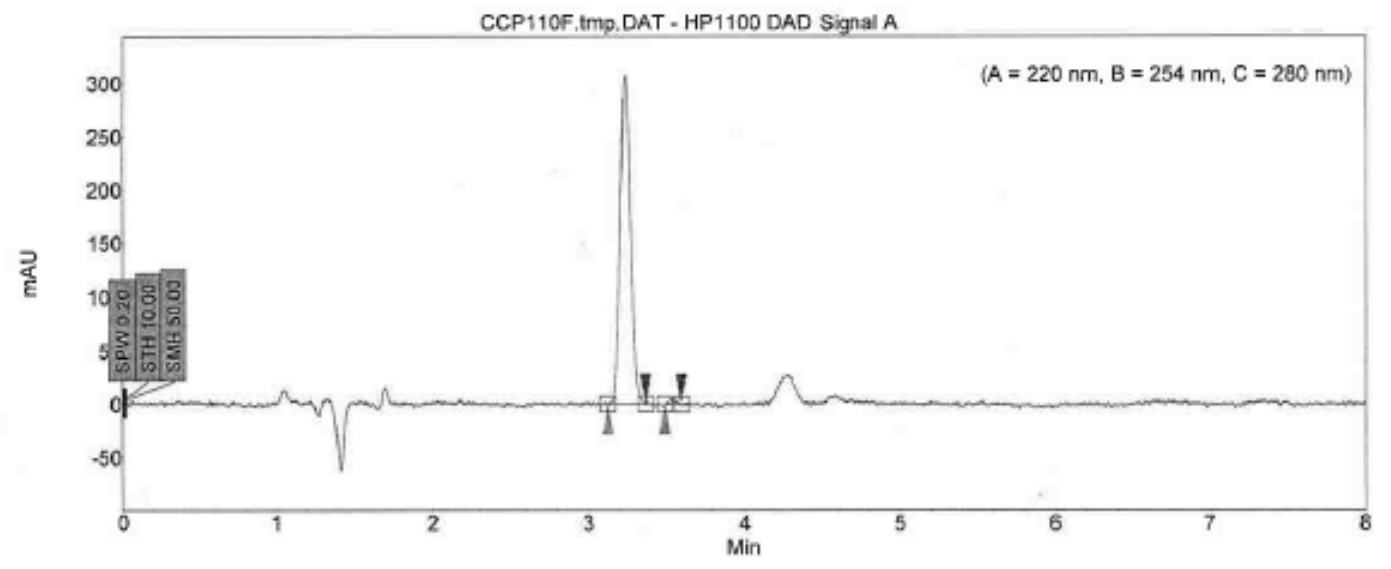

\begin{tabular}{|c|r|r|r|r|r|r|r|}
\hline Index & Time & Width & Height & Res. HW & Selectivity & Area & Area \\
\hline & {$[$ Min] } & {$[$ Min] } & {$[\mu$ ] } & & & [uV.Min] & {$[\%]$} \\
\hline 1 & 3.24 & 0.07 & 307.5 & 0.00 & 0.00 & 23.4 & 99.081 \\
\hline 2 & 3.53 & 0.04 & 4.3 & 3.09 & 1.09 & 0.2 & 0.919 \\
\hline & & & & & & & \\
\hline Total & & & 311.7 & & & 23.7 & 100.000 \\
\hline
\end{tabular}




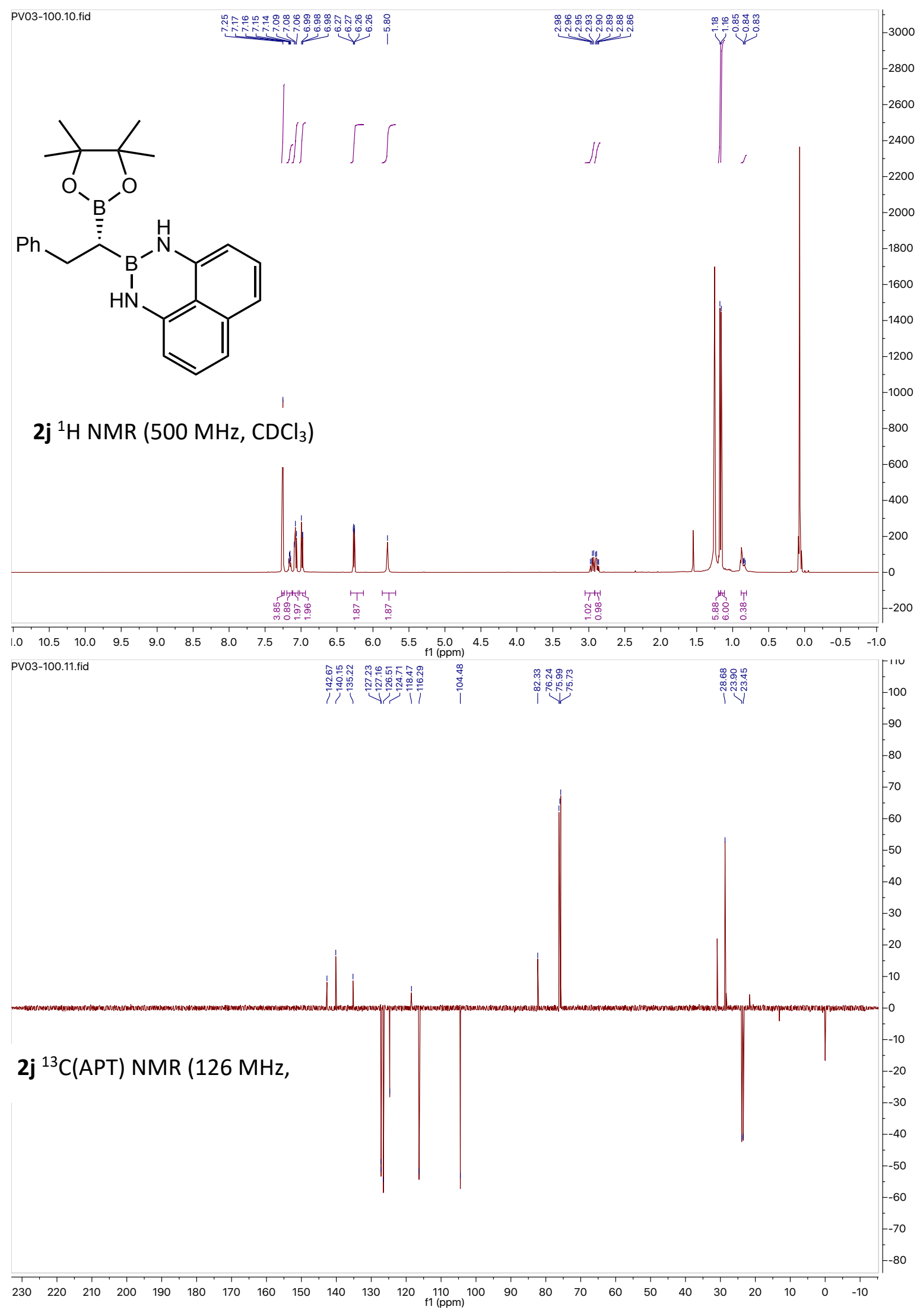




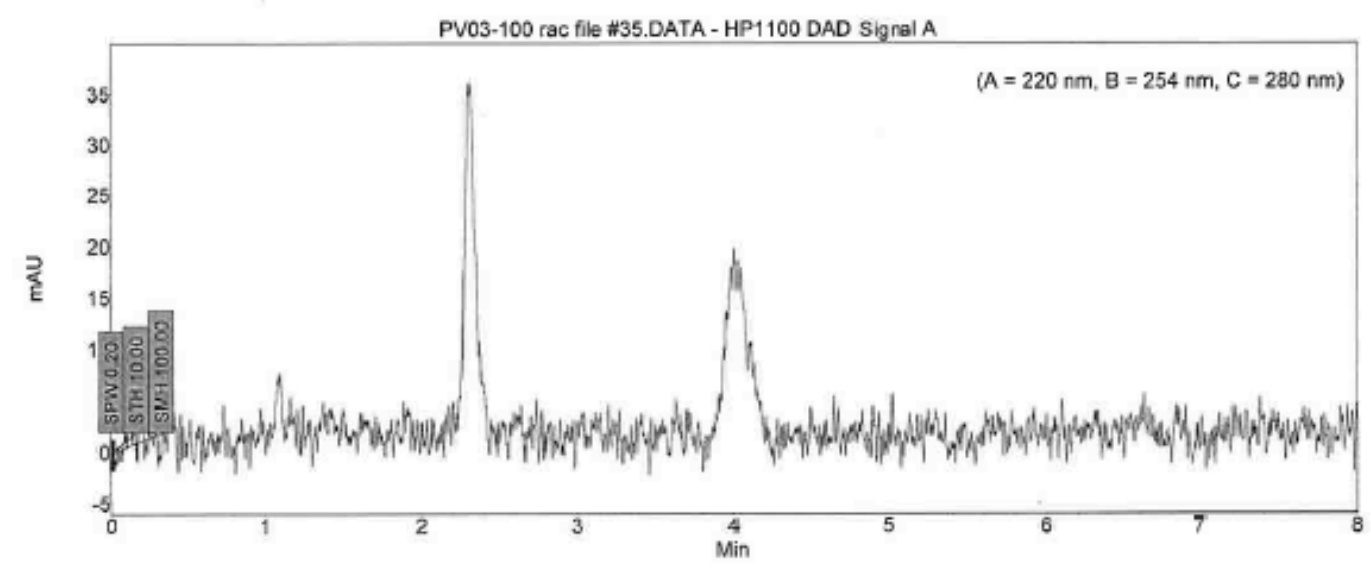

\begin{tabular}{|c|c|r|r|r|r|r|r|}
\hline Index & Time & Width & Height & Res HW & Selectivity & Area & Area \\
\hline \hline & {$[$ Min] } & {$[$ Min] } & {$[\mu \mathrm{V}]$} & & & [uV.Min] & {$[\%]$} \\
\hline & & & & & & & \\
\hline Total & & & 0.0 & & & 0.0 & 0.000 \\
\hline
\end{tabular}

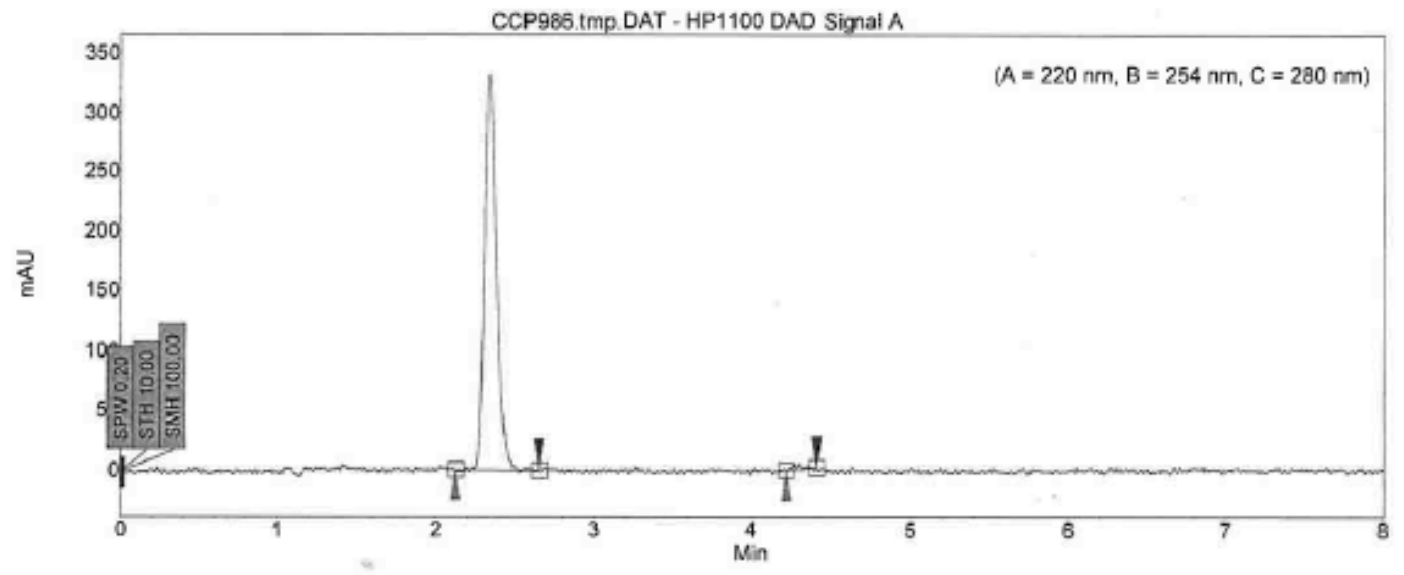

\begin{tabular}{|c|r|r|r|r|r|r|r|}
\hline Index & Time & Width & Height & Res. HW & Selactivity & Area & Area \\
\hline & [Min] & [Min] & {$[\mu \mathrm{M}]$} & & & [uV.Min] & {$[\%]$} \\
\hline 1 & 2.34 & 0.08 & 331.8 & 0.00 & 0.00 & 29.6 & 99.228 \\
\hline 2 & 4.29 & 0.02 & 4.5 & 22.06 & 1.84 & 0.2 & 0.772 \\
\hline & & & & & & & \\
\hline Total & & & 336.3 & & & 29.9 & 100.000 \\
\hline
\end{tabular}




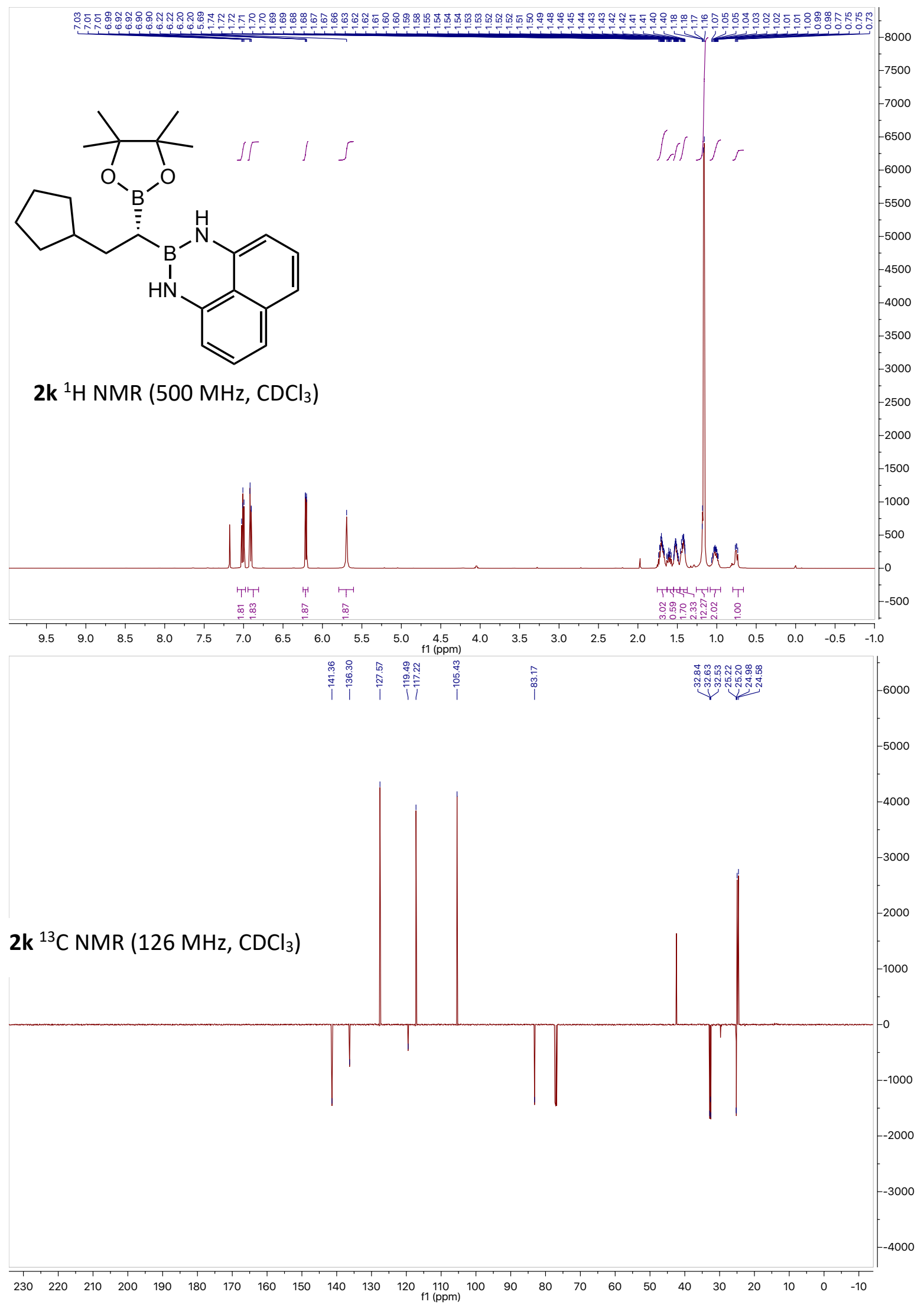




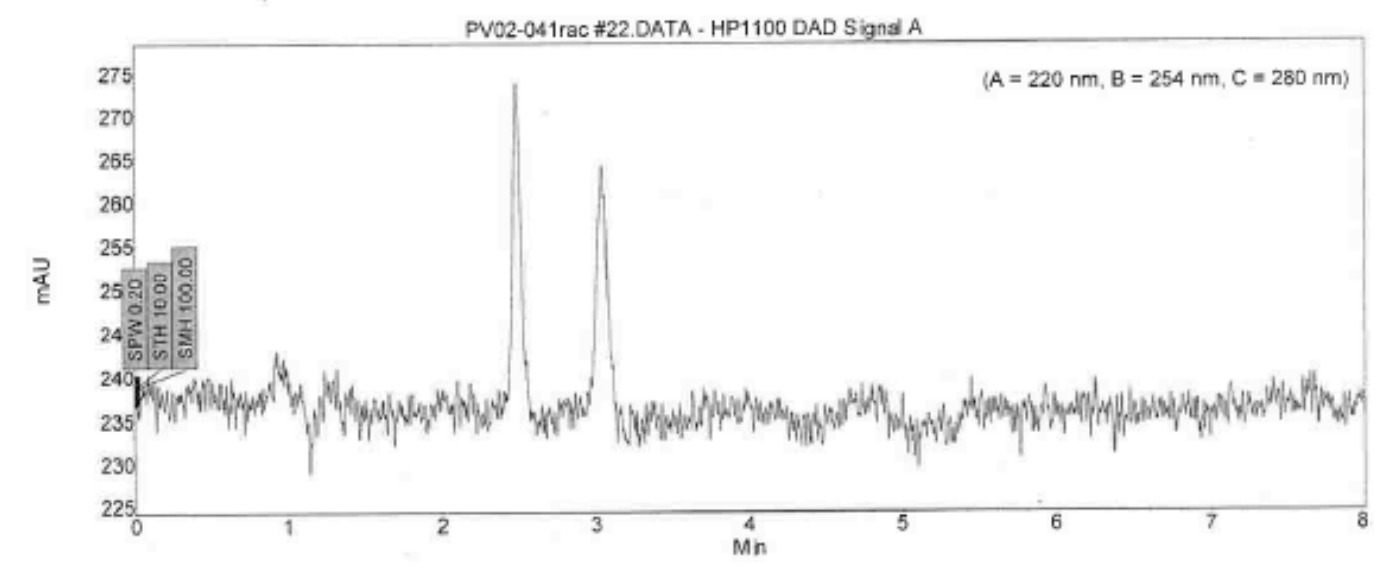

\begin{tabular}{|r|r|r|r|r|r|r|r|}
\hline Index & Time & Width & Height & Res. HW & Selectivity & Area & Area \\
\hline & [Min] & [Min] & [uV] & & & [u/.Min] & {$[\%]$} \\
\hline & & & & & & & \\
\hline Total & & & 0.0 & & & 0.0 & 0.000 \\
\hline
\end{tabular}

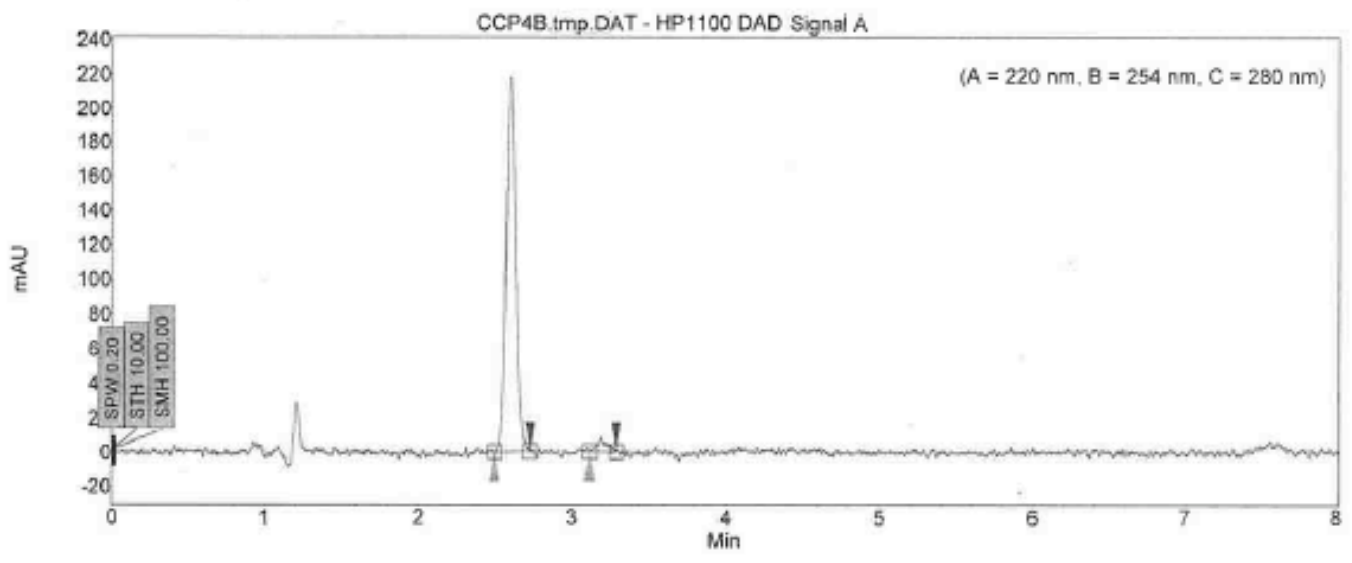

\begin{tabular}{|c|r|r|r|r|r|r|r|}
\hline Index & Time & Width & Height & Res. HW & Selectivity & Area & Avea \\
\hline \hline & Min] & {$[$ Min] } & {$[\mu \mathrm{V}]$} & & & {$[$ UV. Min] } & $(\%)$ \\
\hline 1 & 2.60 & 0.07 & 218.4 & 0.00 & 0.00 & 15.8 & 96.496 \\
\hline 2 & 3.20 & 0.03 & 8.6 & 7.19 & 1.23 & 0.6 & 3.504 \\
\hline & & & & & & & \\
\hline Total & & & 227.0 & & & 16.4 & 100.000 \\
\hline
\end{tabular}




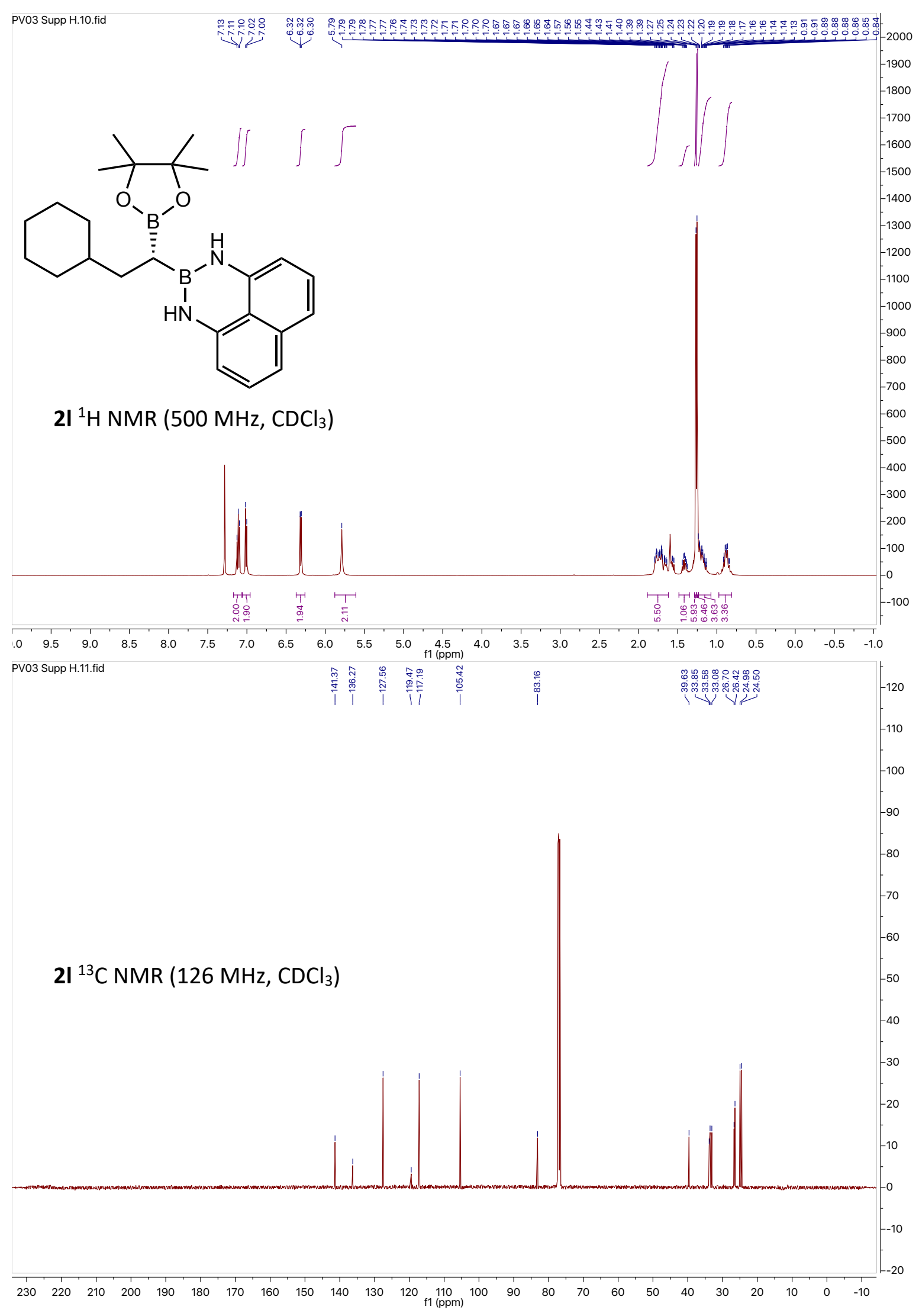


CCP38F tmp.DAT - HP1100 DAD Signal A

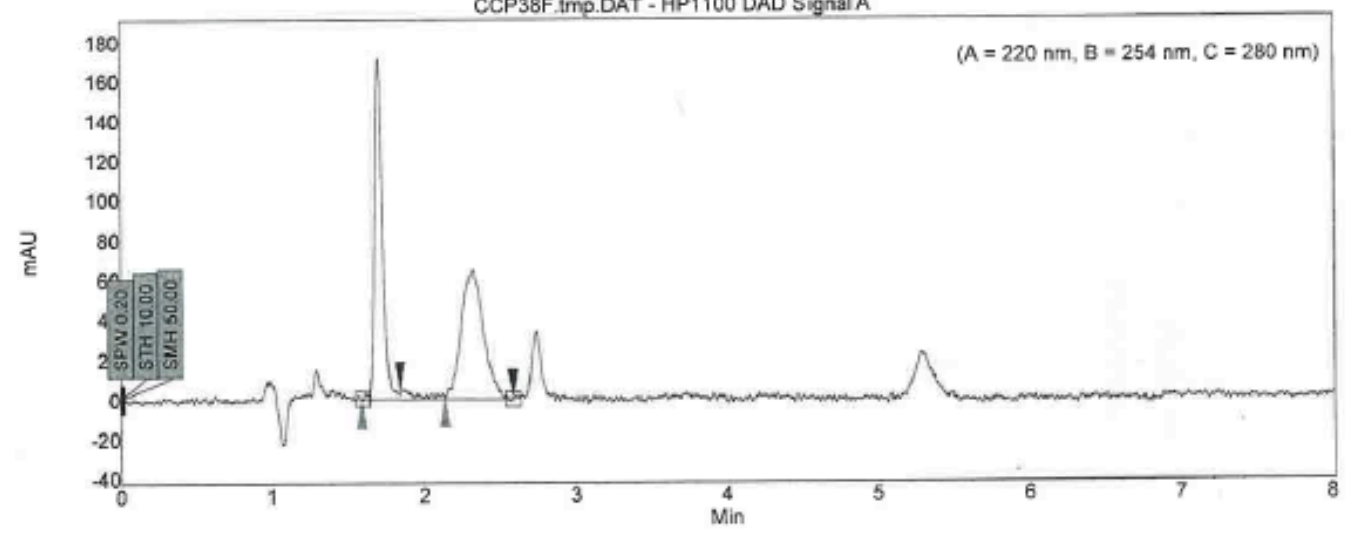

\begin{tabular}{|c|r|r|r|r|r|r|r|}
\hline Index & Time & Widih & Height & Res. HW & Selectivity & Area & Area \\
\hline \hline & {$[$ Min] } & {$[$ Min] } & {$[\mu \mathrm{uV}$} & & & [uV.Min] & {$[\%]$} \\
\hline 1 & 1.70 & 0.06 & 171.5 & 0.00 & 0.00 & 10.7 & 49.467 \\
\hline 2 & 2.31 & 0.16 & 64.3 & 3.41 & 1.36 & 11.0 & 50.533 \\
\hline & & & & & & & \\
\hline Total & & & 235.9 & & & 21.7 & 100.000 \\
\hline
\end{tabular}

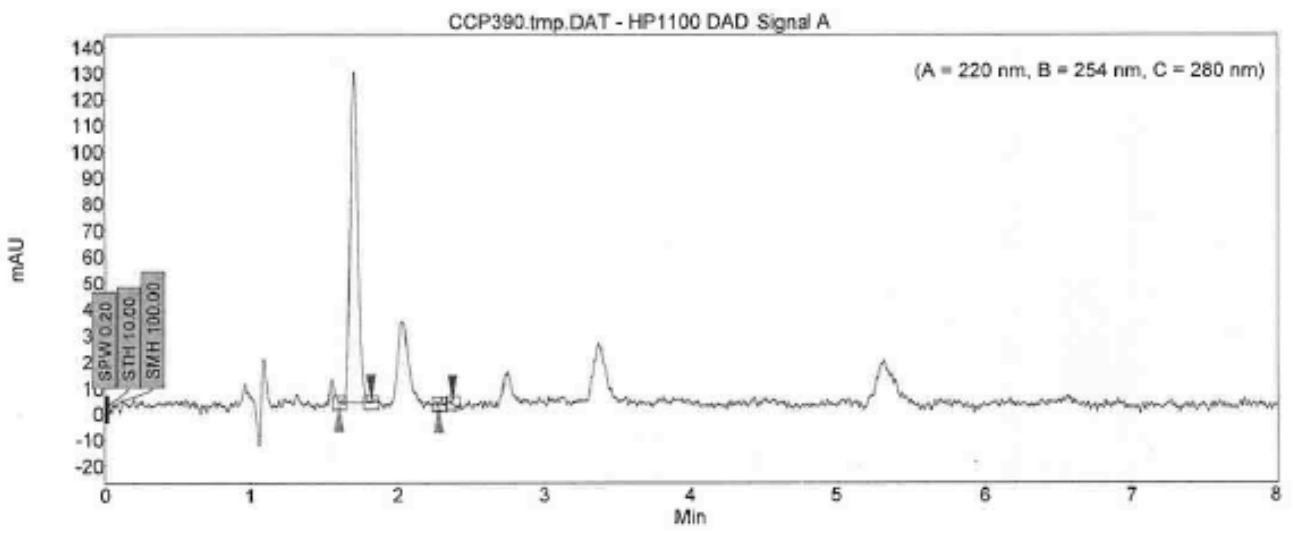

\begin{tabular}{|c|r|r|r|r|r|r|r|}
\hline Index & Time & Width & Height & Res. HW & Selectivity & Area & Area \\
\hline \hline & {$[$ Min] } & {$[$ Min] } & [uM] & & & [uV.Min] & {$[\%]$} \\
\hline 1 & 1.71 & 0.06 & 126.2 & 0.00 & 0.00 & 7.5 & 99.213 \\
\hline 2 & 2.30 & 0.01 & 2.6 & 11.18 & 1.35 & 0.1 & 0.787 \\
\hline & & & & & & & \\
\hline Total & & & 128.8 & & & 7.6 & 100.000 \\
\hline
\end{tabular}




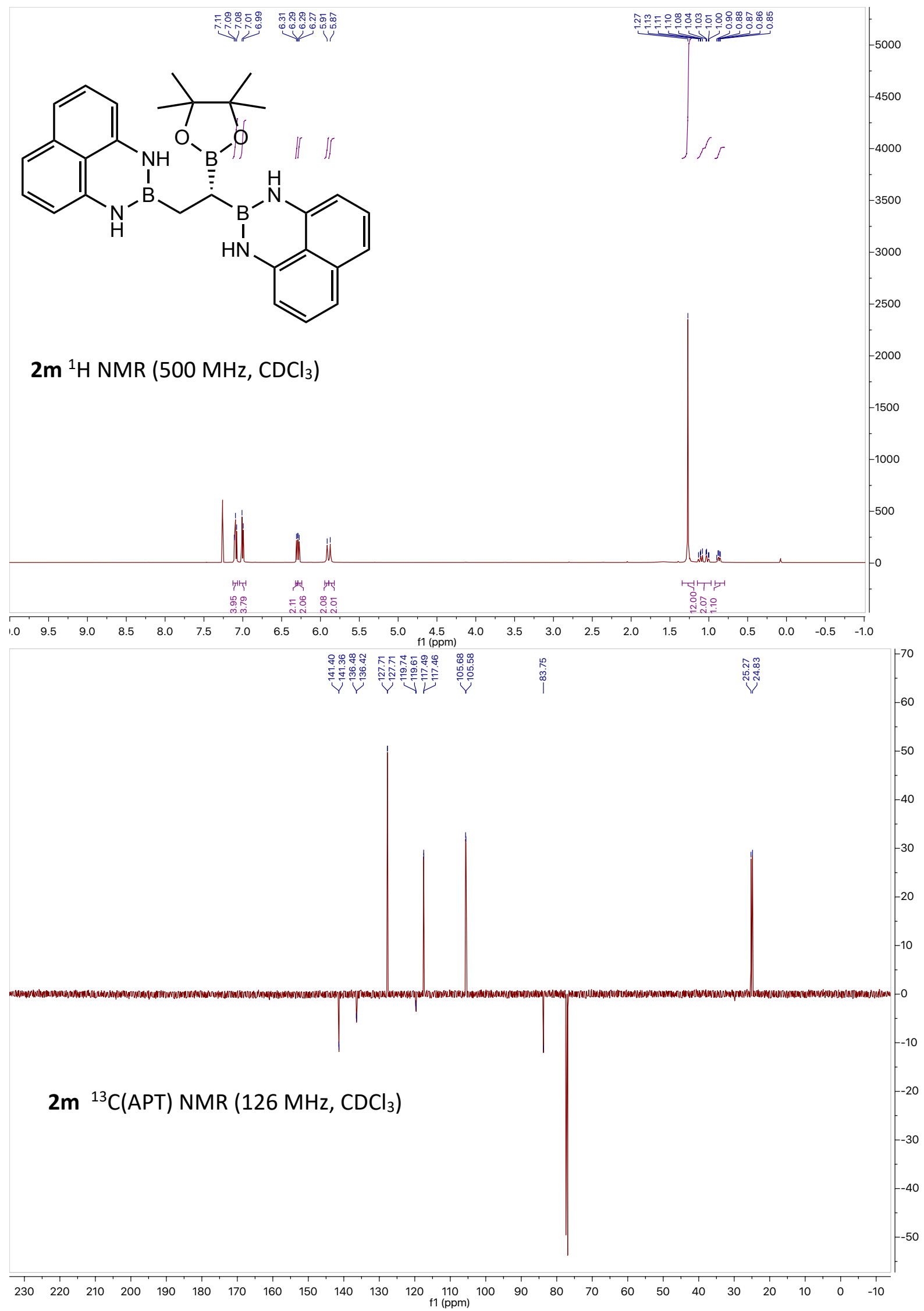




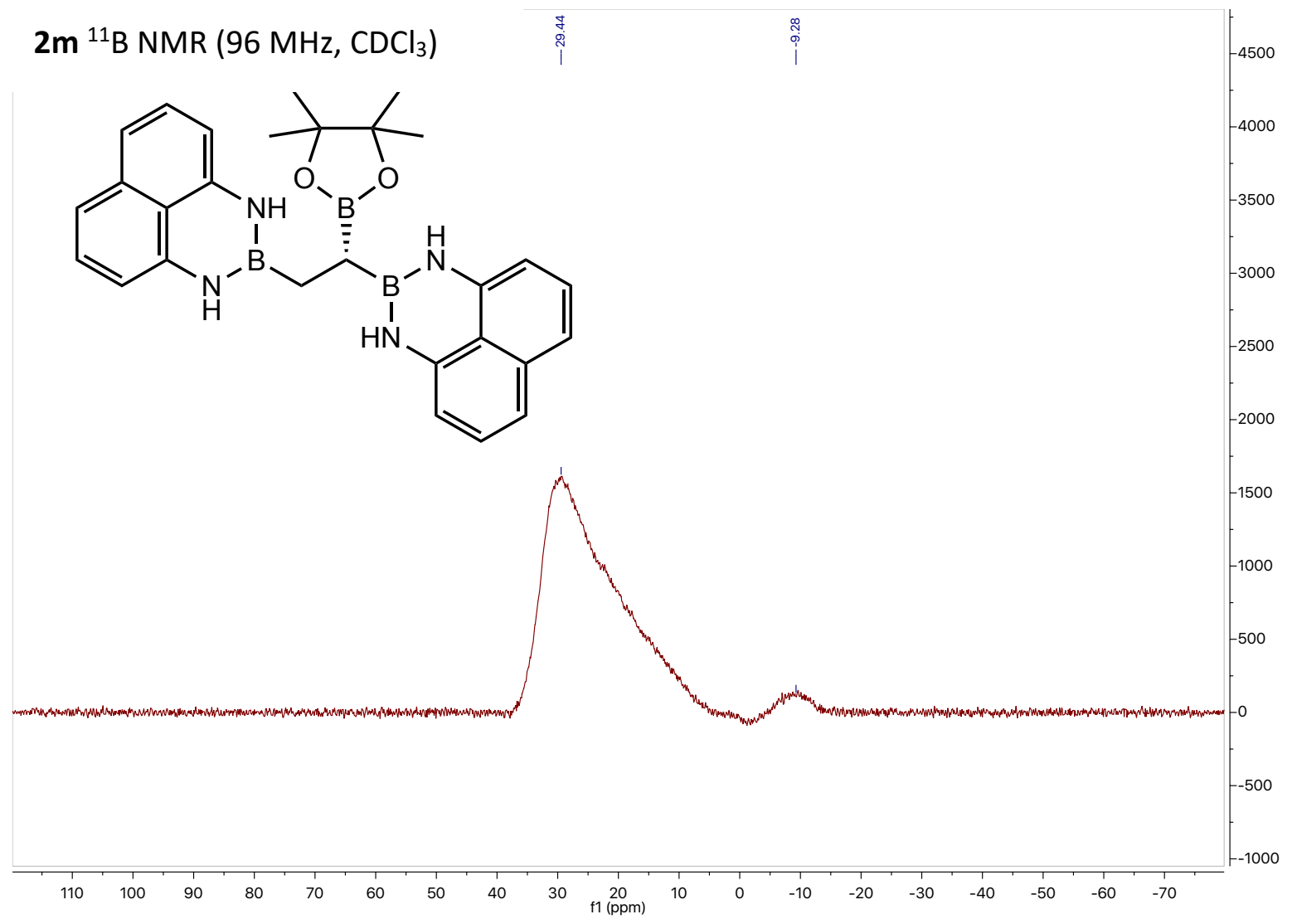




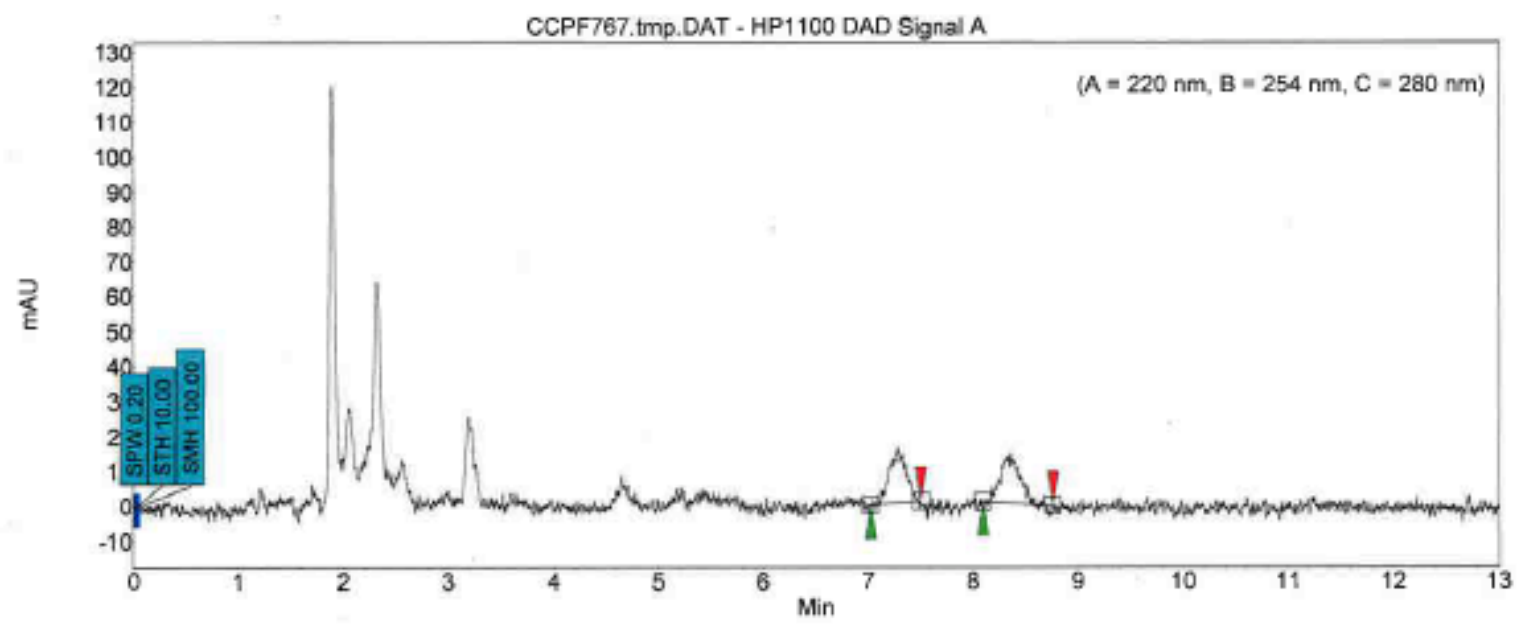

\begin{tabular}{|c|r|r|r|r|r|r|r|}
\hline Index & Time & Width & Height & Res. HW & Selectivity & Area & Area \\
\hline \hline & {$[$ Min] } & {$[$ Min] } & {$[\mu \mathrm{M}]$} & & & {$[\mu \mathrm{V} \cdot \mathrm{Min}]$} & {$[\%]$} \\
\hline 1 & 7.28 & 0.18 & 16.1 & 0.00 & 0.00 & 2.8 & 50.749 \\
\hline 2 & 8.36 & 0.16 & 14.5 & 3.72 & 1.15 & 2.7 & 49.251 \\
\hline & & & & & & & \\
\hline Total & & & 30.6 & & & 5.6 & 100.000 \\
\hline
\end{tabular}

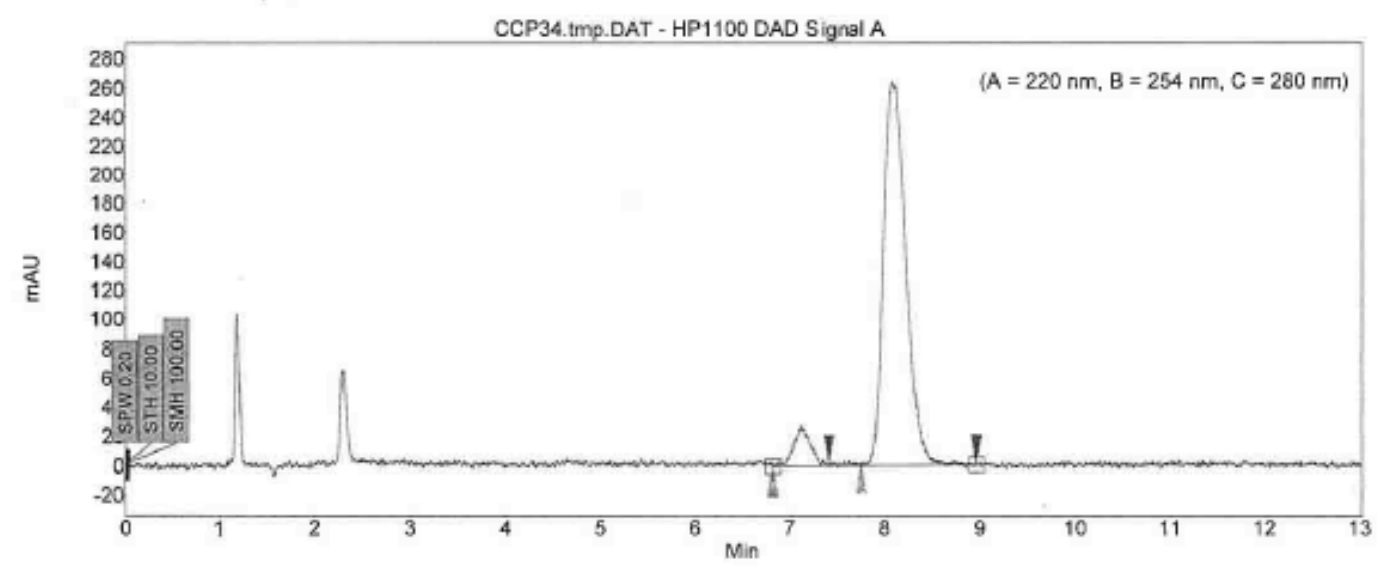

\begin{tabular}{|c|r|r|r|r|r|r|r|}
\hline Index & Timo & Width & Height & Res. HW & Selectivity & Area & Area \\
\hline & {$[$ Min] } & {$[$ Min] } & {$[\mu \mathrm{W}]$} & & & [uV.Min] & {$[\%]$} \\
\hline 1 & 7.10 & 0.19 & 27.9 & 0.00 & 0.00 & 5.6 & 7.291 \\
\hline 2 & 8.05 & 0.25 & 264.0 & 2.55 & 1.13 & 712 & 92.709 \\
\hline & & & & & & & \\
\hline Total & & & 291.8 & & & 76.9 & 100.000 \\
\hline
\end{tabular}




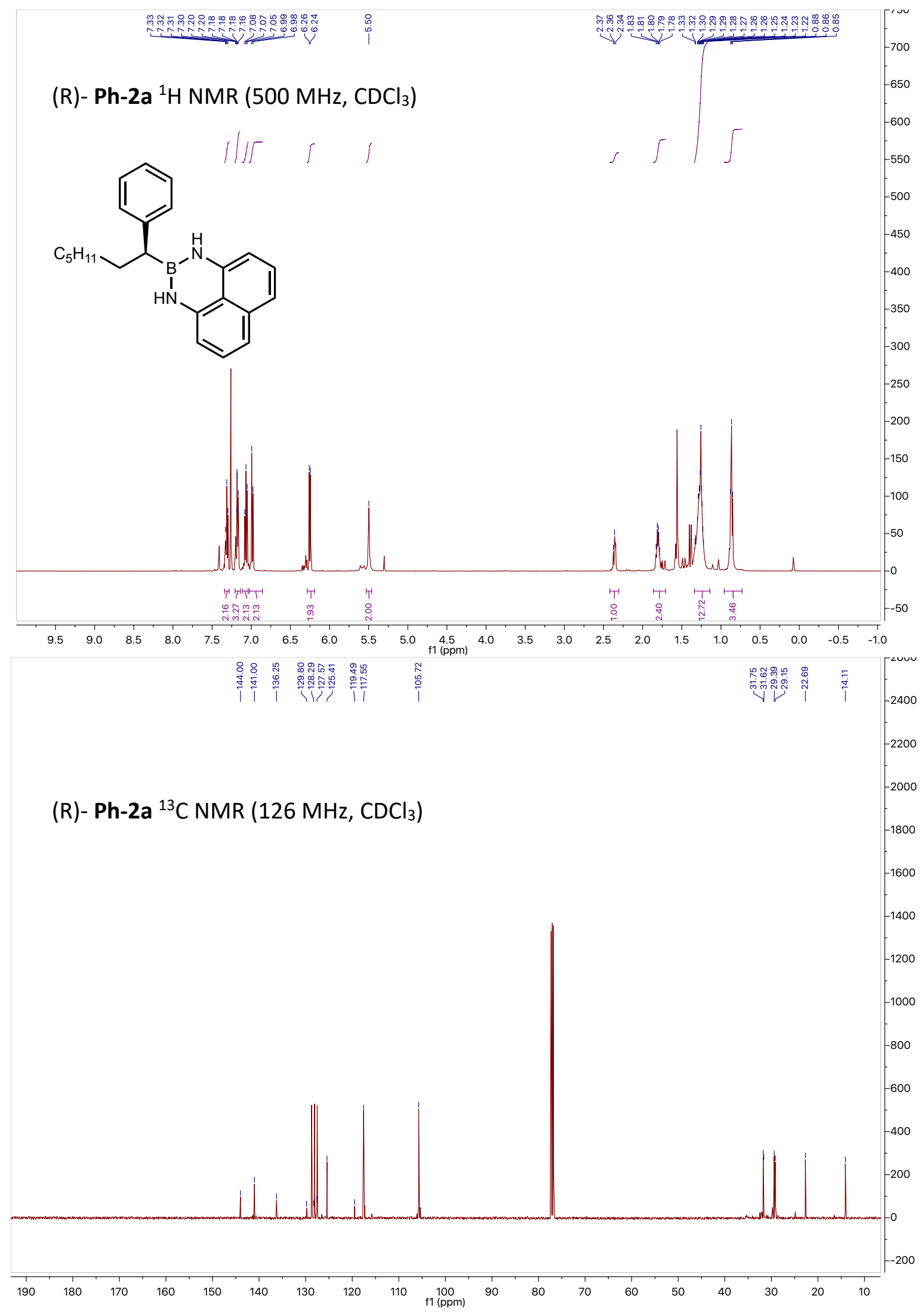




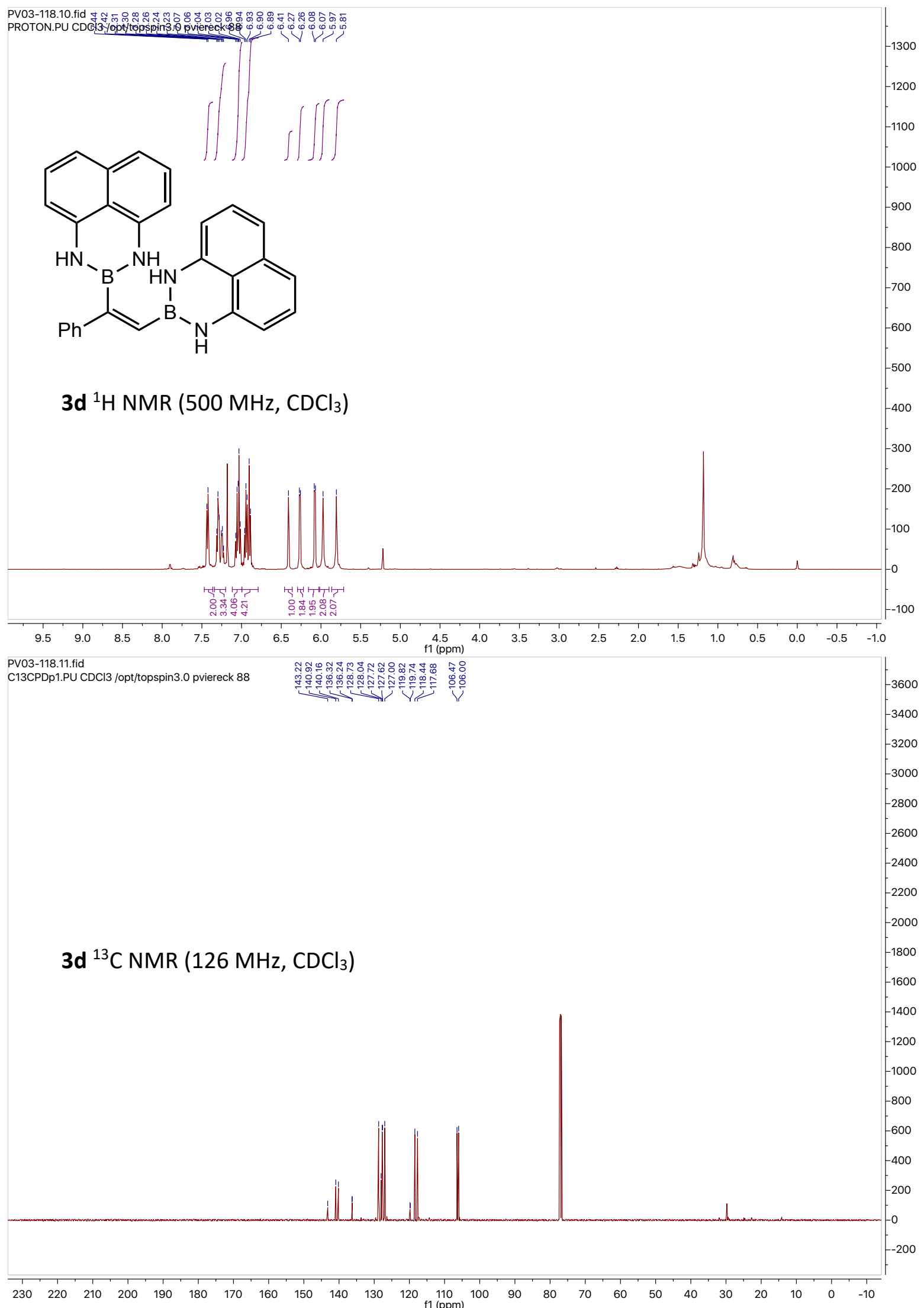



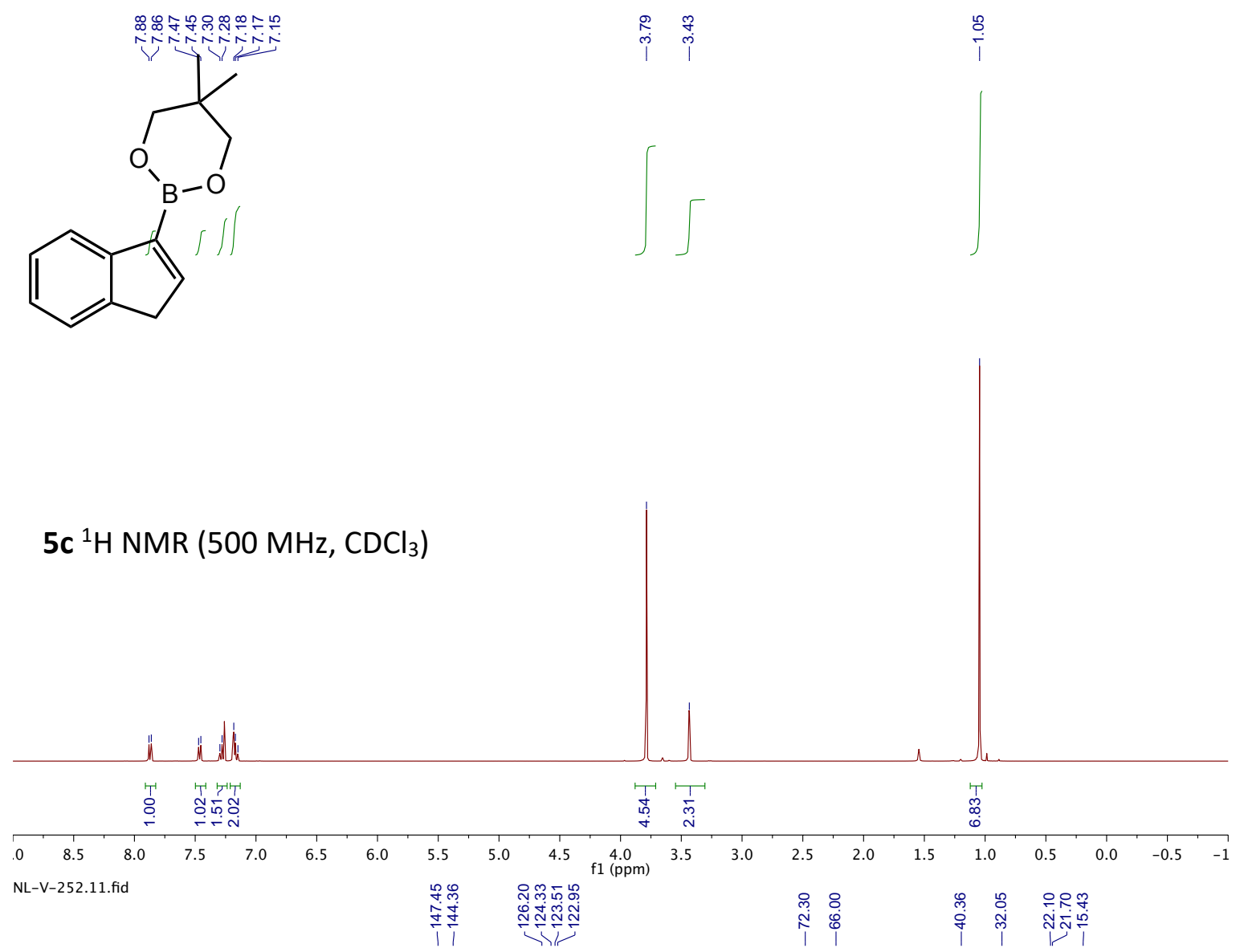

$5 \mathrm{c}^{13} \mathrm{C}$ NMR $\left(126 \mathrm{MHz}, \mathrm{CDCl}_{3}\right)$

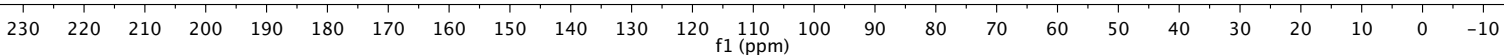



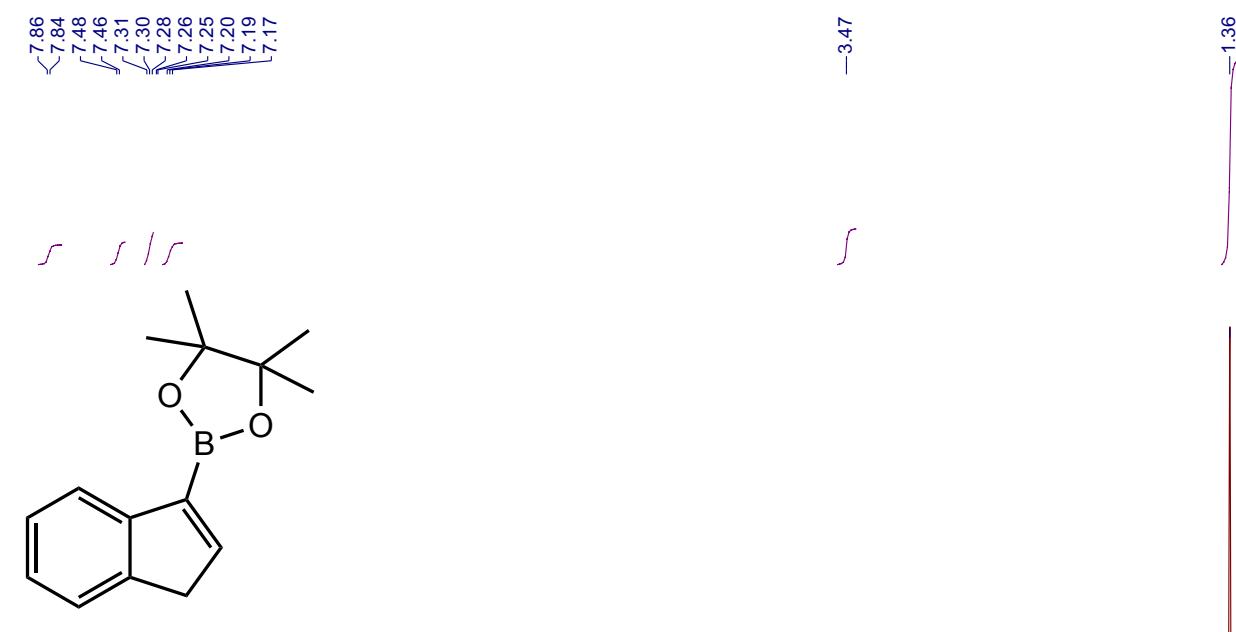

5d ${ }^{1} \mathrm{H}$ NMR $\left(500 \mathrm{MHz}, \mathrm{CDCl}_{3}\right)$

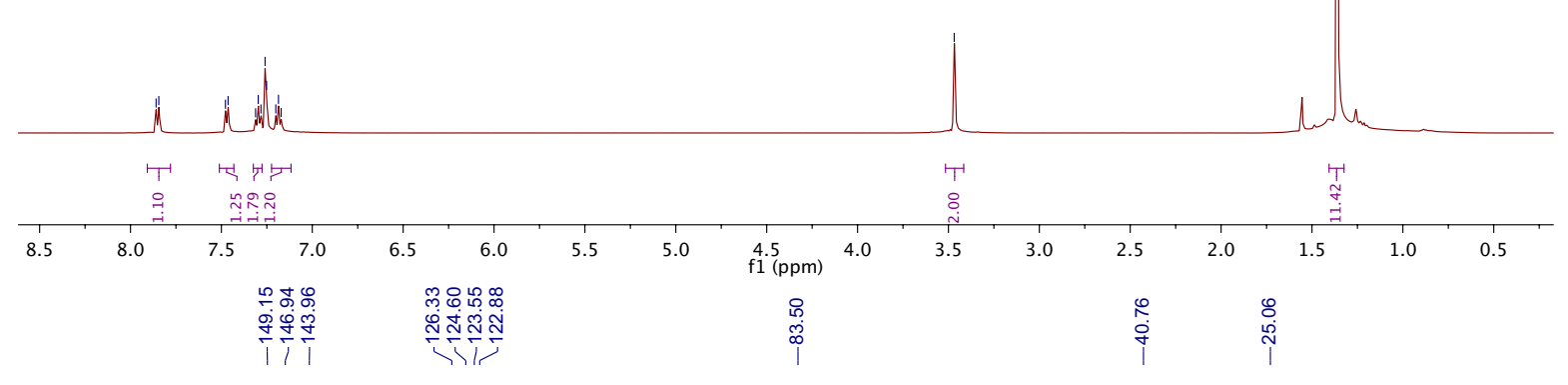

5d ${ }^{13} \mathrm{C} \mathrm{NMR}\left(126 \mathrm{MHz}, \mathrm{CDCl}_{3}\right)$

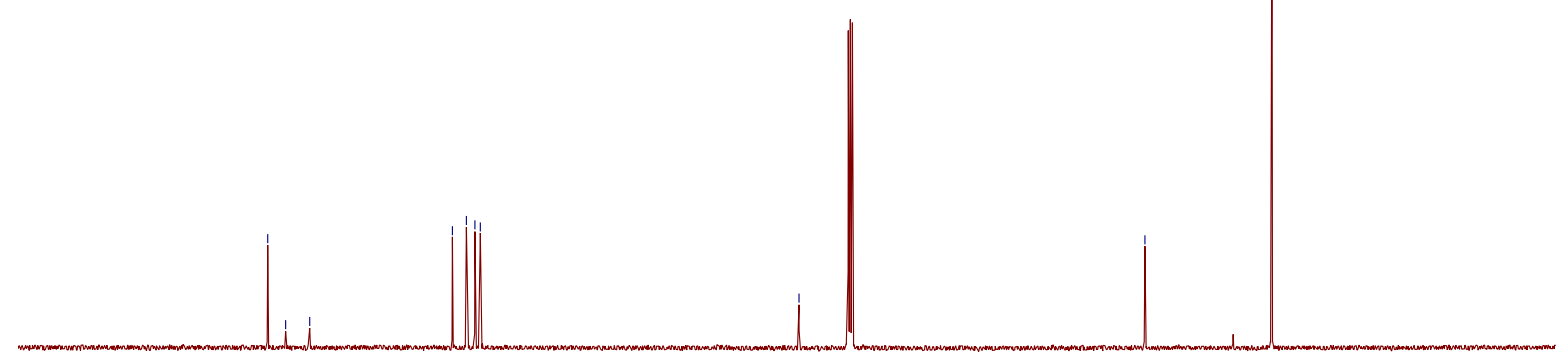

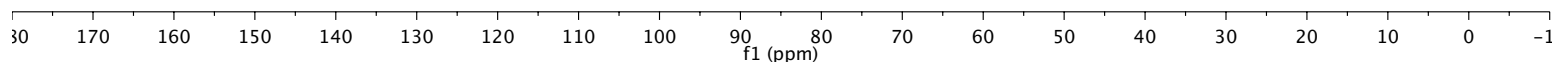




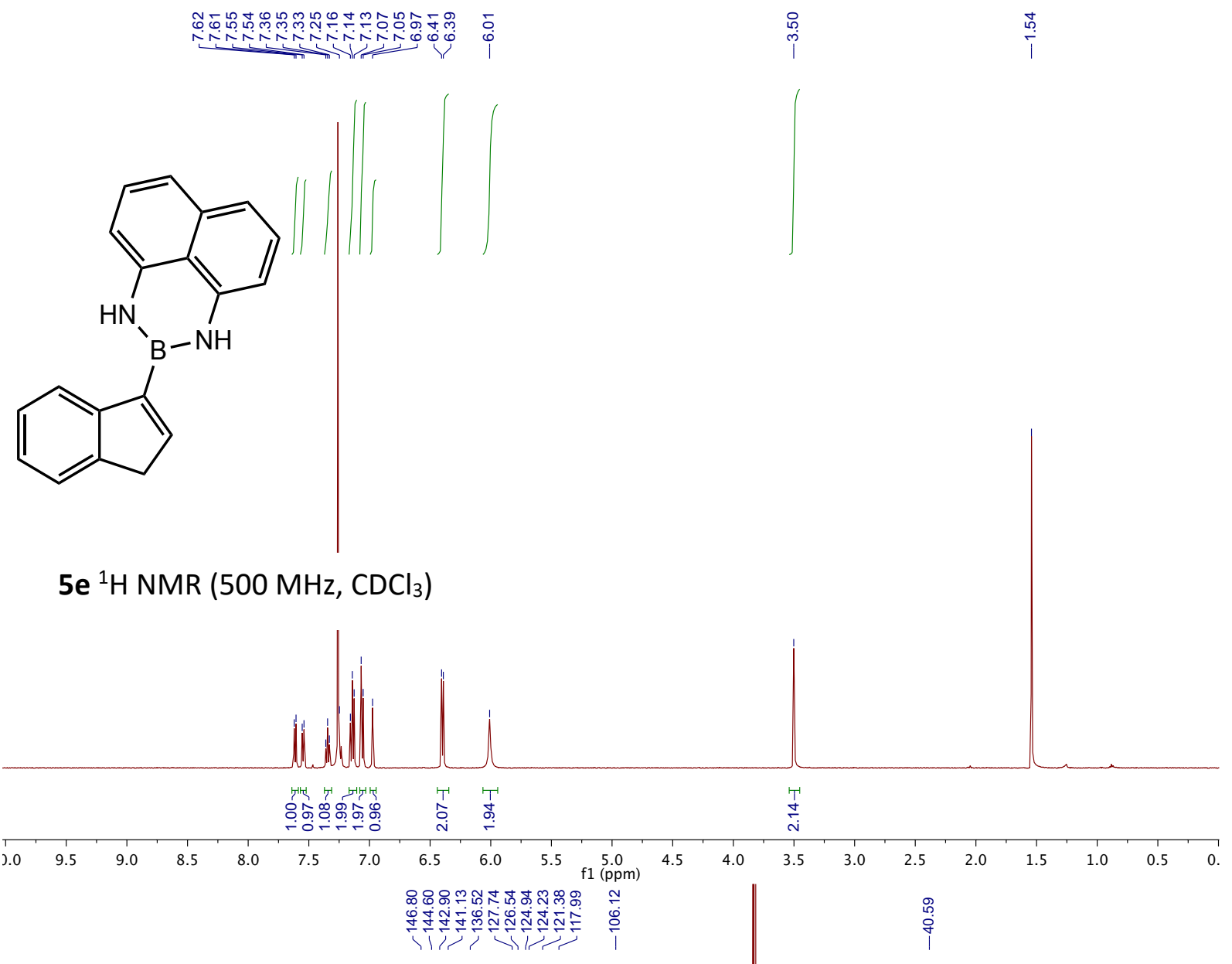

$5 e^{13} \mathrm{C}$ NMR $\left(126 \mathrm{MHz}, \mathrm{CDCl}_{3}\right)$

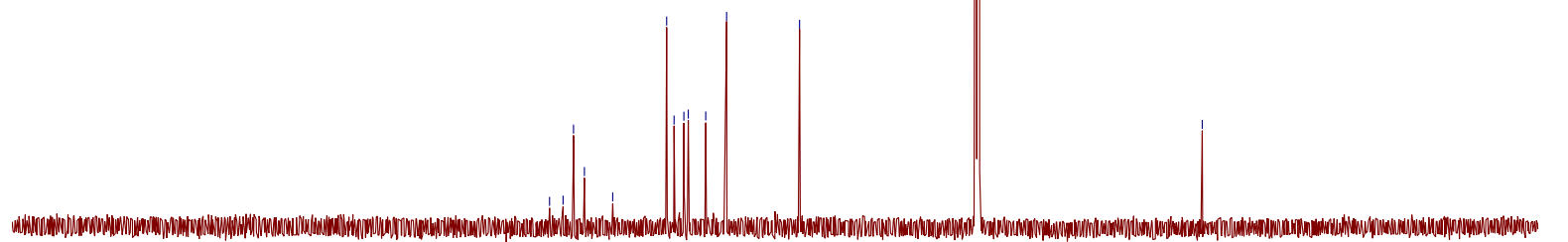

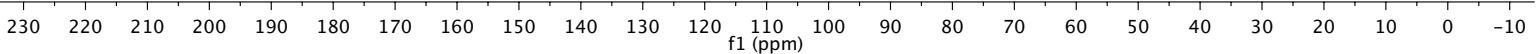




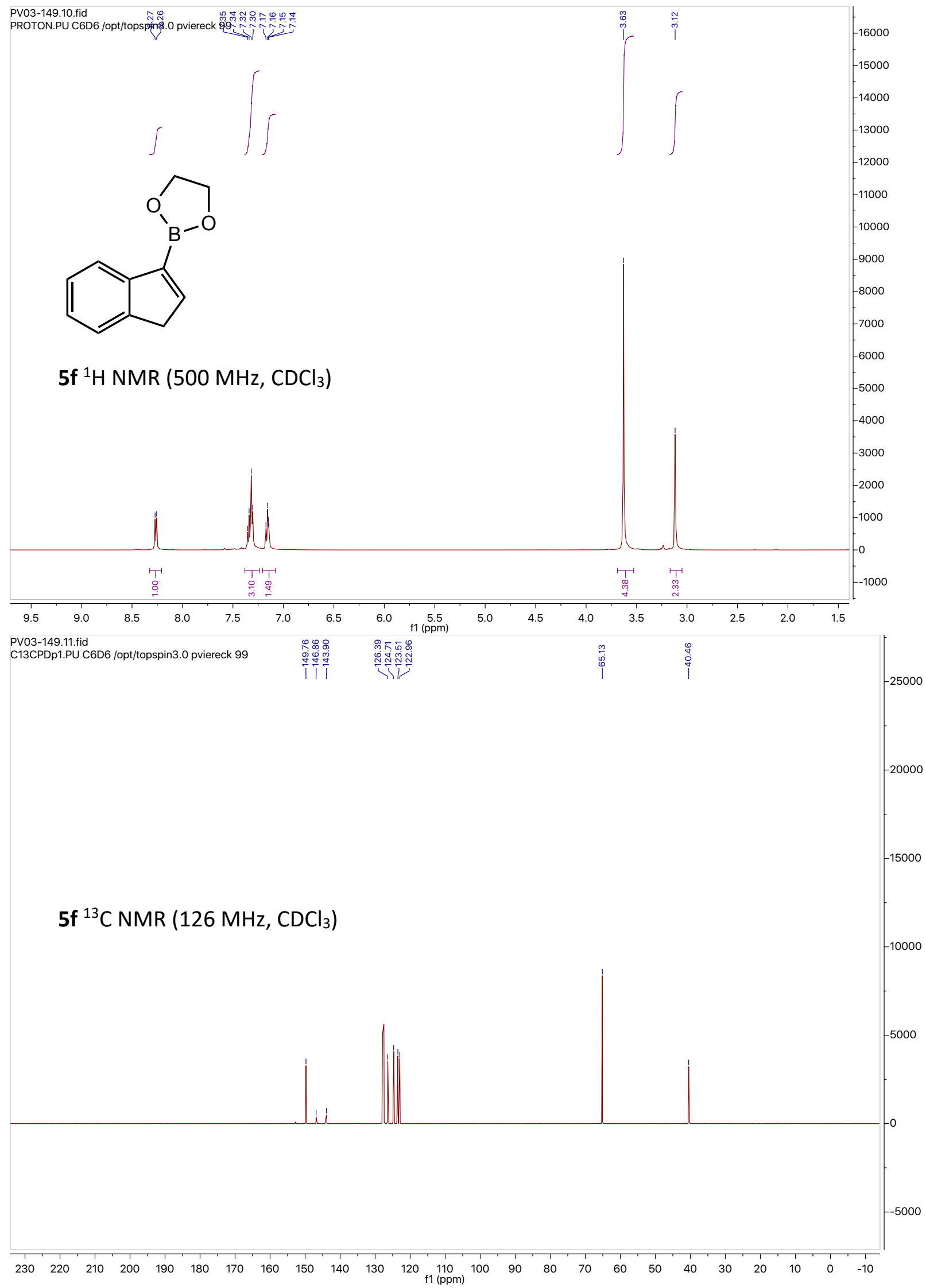




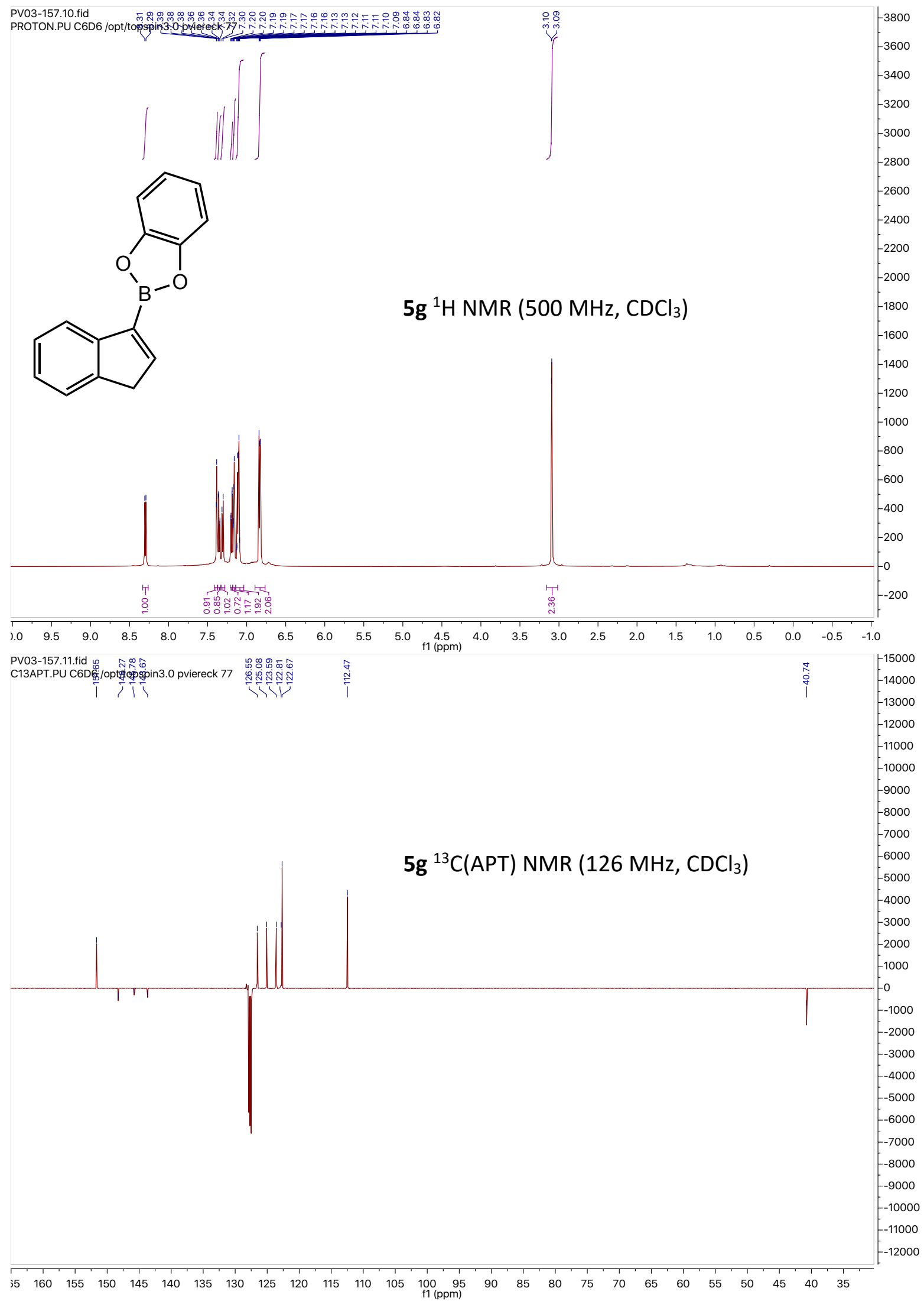

